\title{
Comparative pathogenomics in the ascomycete plant pathogens \\ Zymoseptoria tritici and Verticillium dahliae
}

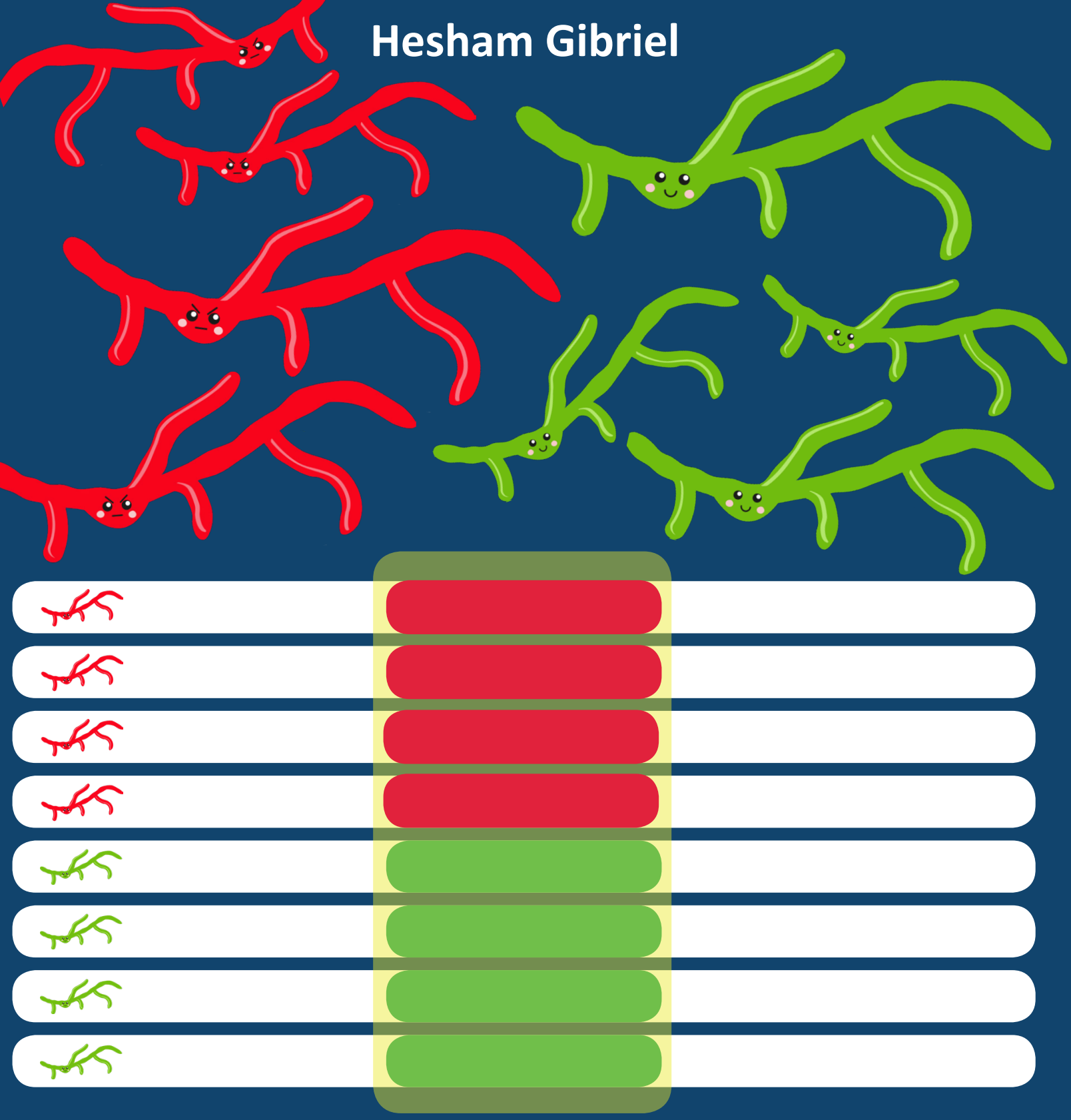




\section{Propositions}

1. Improper gene annotation of Zymoseptoria tritici hampered the discovery of AvrStb6 for years.

(this thesis)

2. Verticillium dahliae strains infecting the same host plant utilize divergent effector repertoires.

(this thesis)

3. Artificial insulin-releasing cells may be the key therapy for diabetes in the future.

4. Establishments of networks between weather forecasters in the developed world and governmental organizations in developing regions can diminish the impact of future weather disasters.

5. Scientists should engage in reporting their research results publicly on social media to raise awareness of the value of science.

6. Obesity should not be blamed on genes, but on nutritional habits.

7. Considering the availability of vast amounts of solar energy in the Middle East, solar energy should become the main source of power for the region.

8. Bodybuilding athletes should consider healthy diets as replacement for protein supplements.

Propositions belonging to the thesis, entitled

'Comparative pathogenomics in the ascomycete plant pathogens

Zymoseptoria tritici and Verticillium dahliae'.

Hesham Gibriel

Wageningen, 20 February 2019 
Comparative pathogenomics in the ascomycete plant pathogens Zymoseptoria tritici and Verticillium dahliae

Hesham Gibriel 


\section{Thesis committee}

\section{Promotor}

Prof. Dr B.P.H.J. Thomma

Professor of Phytopathology

Wageningen University \& Research

\section{Co-promotors}

Dr M.F. Seidl

Assistant Professor, Laboratory of Phytopathology

Wageningen University \& Research

Prof. Dr G.H.J. Kema

Special Professor Tropical Phytopathology

Wageningen University \& Research

\section{Other members}

Prof. Dr J.E. Kammenga, Wageningen University \& Research

Dr A. Sanchez-Vallet, ETH Zurich, Switzerland

Dr M.H. Medema, Wageningen University \& Research

Dr Ronnie de Jonge, Utrecht University

This research was conducted under the auspices of the Graduate School Experimental Plant Sciences. 


\title{
Comparative pathogenomics in the ascomycete plant pathogens Zymoseptoria tritici and Verticillium dahliae
}

\author{
Hesham Gibriel
}

Thesis

submitted in fulfilment of the requirements for the degree of doctor at Wageningen University

by the authority of the Rector Magnificus, Prof. Dr A.P.J. Mol, in the presence of the

Thesis Committee appointed by the Academic Board to be defended in public on Wednesday 20 February 2019 at 4 p.m. in the Aula. 
Hesham Gibriel

Comparative pathogenomics in the ascomycete plant pathogens

Zymoseptoria tritici and Verticillium dahliae, 238 pages.

PhD thesis, Wageningen University, Wageningen, The Netherlands (2019) With references, with summary in English

DOI: $10.18174 / 467538$

ISBN: 978-94-6343-572-7 


\section{Table of contents}

$\begin{array}{lll}\text { Chapter } 1 & \text { General introduction } & 7\end{array}$

Chapter 2 The age of effectors: genome-based discovery and 21 applications

Chapter 3 Verticillium dahliae strains that infect the same host plant 43 display highly divergent effector catalogs

Chapter 4 The Verticillium dahliae effector Sun1 quantitatively 77 contributes to virulence on sunflower

Chapter 5 Signatures of adaptation to bread or durum wheat in a global 107 collection of Zymoseptoria tritici isolates

Chapter 6 Stress and sexual reproduction affect the dynamics of the 153 wheat pathogen effector AvrStb6 and strobilurin resistance

Chapter 7 General discussion: Effector repertoires of plant pathogens 209 revealed by comparative genomics

Summary

Acknowledgements

About the author

List of publications 


\section{Chapter 1}

$$
\text { do }
$$

$$
26
$$


General introduction 



\section{HOST-MICROBE INTERACTIONS}

Genomes are highly variable and differ significantly between, and even within, species. This genetic variability is established by a variety of mechanisms ranging from single nucleotide polymorphisms (SNPs) to large-scale structural variations, and provides the basis for species to continuously evolve under changing environmental conditions (Seidl and Thomma 2014). This is particularly relevant for microbial pathogens as they are engaged in co-evolutionary arms races with their hosts, where the pathogen tries to establish the parasitic relationship while the host tries to ward off the pathogen (Dong et al. 2015; Raffaele and Kamoun 2012; Seidl and Thomma 2014).

Microbial plant pathogens cause devastating diseases on important crops, leading to billions of dollars of losses annually and threatening global food security (Fisher et al. 2012). To defend themselves against invaders, plants have evolved extracellular and intracellular receptors that can detect invading microbes through recognition of socalled invasion patterns that betray the presence or activity of an invader (Cook et al. 2015). To establish themselves, invaders secrete so-called effectors that suppress host immune responses and facilitate the symbiosis (Cook et al. 2015; Rovenich et al. 2014). However, also effectors can act as invasion patterns when they are detected by plant immune receptors (Cook et al. 2015). Therefore, pathogens need to continuously diversify their effector repertoires to avoid recognition by the plant immune system by evolving novel effectors, or losing or modifying existing ones, while the plant simultaneously evolves novel receptors to intercept pathogens (Jones and Dangl 2006). Thus, plants and pathogens are engaged in co-evolutionary arms races that shape their evolution. It can be anticipated that this co-evolution ultimately leads to highly specialized pathogens that can only infect few or even a single host. However, some pathogens are successful pathogens of a broad range of hosts. Such broad host range pathogens, arguably, have to engage in arms races with multiple hosts. This leads to the question how broad host range pathogens are able to adapt to be competitive in the arms race with multiple hosts.

In my research, I have used two ascomycete plant pathogens in order to study their adaptation on a narrow versus a broad host range. More specifically, I focussed on Zymoseptoria tritici that has a narrow host range with wheat as its major host although it occasionally infects other grasses as well. Additionally, I studied Verticillium dahliae that is able to colonize a broad range of plant hosts, encompassing hundreds of species (Figure 1). 

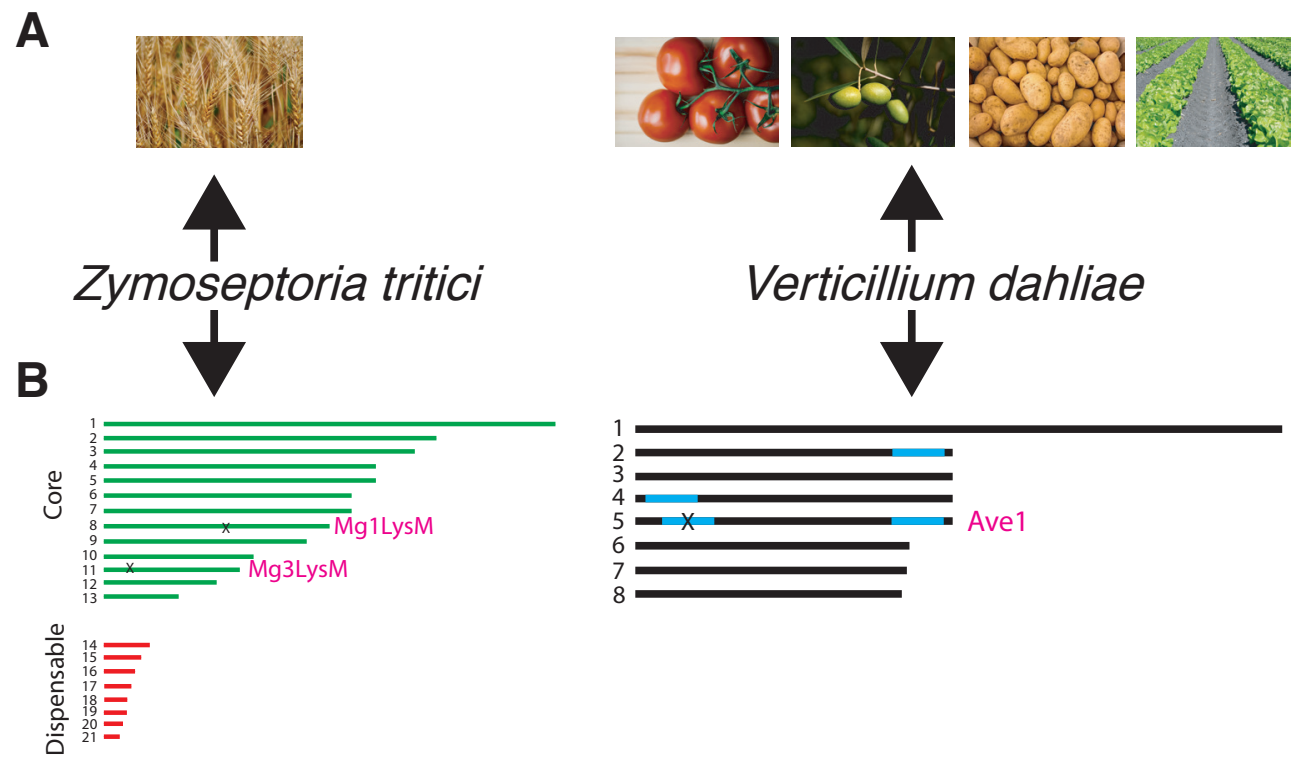

Figure 1. Two selected ascomycete plant pathogens used in this thesis, which have divergent host ranges and encode effector genes located on different genomic regions. (A) On the left side, the fungal wheat pathogen $Z$. tritici infects only few host plants, while on the right side $V$. dahliae colonizes a diverse host species including tomato, olive, potato, and lettuce. (B) The genomes and the location of a selection of previously characterized effector genes of these two pathogens are shown. On the left side, the genome of $Z$. tritici is shown that contains 13 core chromosomes (green) and eight dispensable ones (red). Effector genes in Z. tritici are located on the core chromosomes. For example, the effector genes $M g 3 L y s M$ and Mg1LysM are located on chromosome 11 and chromosome 8, respectively. No effector genes are known to reside on the dispensable ones. On the right side, the genome of $V$. dahliae is shown, which contains 8 chromosomes. This pathogen contains lineage-specific regions (blue), which encode effector genes. For example, the effector gene Ave 1 is localized within such an LS region.

\section{THE FUNGAL WHEAT PATHOGEN ZYMOSEPTORIA TRITICI}

Wheat is one of the world's major crops that provides about $20 \%$ of all calories consumed by people worldwide (Shiferaw et al. 2013). However, wheat is susceptible to a variety of pathogens including the notorious fungal pathogen $Z$. tritici that causes Septoria tritici leaf blotch (STB). This disease is considered the economically most damaging disease of wheat worldwide (Fones and Gurr 2015). In Europe, the disease leads to 1 billion euro losses per year (Kettles and Kanyuka 2016). STB disease management strongly relies on fungicide usage (Torriani et al. 2015). However, the extensive use of fungicides has resulted in the emergence of fungicide resistant $Z$. tritici strains over the last decades (Cools and Fraaije 2008). Therefore, screening for STB resistance in wheat germplasm 
is an important cornerstone for disease management. To date, 21 Stb resistance genes have been genetically mapped (Brown et al. 2015). Stb6, which is a common resistance gene that is exploited in European wheat cultivars (Chartrain et al. 2005), is the first resistance gene that was recently cloned (Saintenac et al. 2018).

The life cycle of $Z$. tritici includes both sexual and asexual reproduction. Sexual reproduction involves the production of air-borne sexual ascospores year-round with peaks at the onset of the growing season, thus providing genetically diverse $Z$. tritici populations (Hunter et al. 1999). Subsequently, asexual conidia are produced and disseminated over short distances (Wittenberg et al. 2009). The infection process of Z. tritici on wheat plants is initiated by sexual ascospores or asexual pycnidiospores that land onto the leaf surface by rain splash. Subsequently, spores germinate and the fungus colonizes the apoplastic space between mesophyll cells without apparent damage to host cells (Ponomarenko et al. 2011). This symptomless phase is referred to as the biotrophic phase. After 7-10 days, the fungus switches to a symptomatic phase, referred to as necrotrophic, where lesions appear in which pycnidia develop (Ponomarenko et al. 2011).

Z. tritici is pathogenic on both bread wheat (Triticum aestivum) and durum wheat ( $T$. turgidum) cultivars (Annone et al. 1996). However, individual Z. tritici isolates exhibit strong host species specificity (Kema and van Silfhout 1997). This means that the majority of $Z$. tritici isolates originating from durum wheat are virulent on the majority of durum wheat cultivars, but avirulent on bread wheat cultivars, and vice versa. Moreover, individual Z. tritici isolates exhibit cultivar specificity, meaning that Z. tritici isolates are virulent or avirulent on a particular cultivar within a wheat species. In this manner, Z. tritici isolate IPO94269 is virulent on bread wheat cultivar Shafir that carries Stb6, while Z. tritici isolate IPO323 is avirulent on this cultivar. However, so far, Z. tritici gene(s) associated with host and cultivar species specificity have not been identified.

\section{Evolution of virulence in Z. tritici}

Advances in high-throughput sequencing technologies have facilitated rapid sequencing of entire genomes of fungal plant pathogens (Metzker 2010). This has allowed phytopathologists to gain insight into the evolution of virulence by studying genomic plasticity and determining effector catalogs. The genome of isolate IPO323 was the first Z. tritici isolate to be sequenced. It has a size of $39.69 \mathrm{Mb}$, divided over 21 chromosomes (Goodwin et al. 2011). Notably, Z. tritici has 13 core chromosomes (CCs, chromosome 1-13) that are present in all isolates, while eight chromosomes are considered to be conditionally dispensable chromosomes (CDCs, chromosome 14-21). These latter can be absent in an isolate without an obvious effect on fungal fitness (Goodwin et al. 2011). Over the past few years, there has been a growing interest to investigate the origin and role of these CDCs in the pathogenicity of $Z$. tritici. A comparative genomics study of species closely related to $Z$. tritici has suggested that at least some CDCs originated prior to the emergence of $Z$. tritici as a specialized wheat pathogen (Stukenbrock et al. 2010). 
Moreover, it has been shown that CDCs can undergo chromosome fusions, resulting in newly formed chromosomes, and thus can contribute to the rapid adaptive evolution (Croll et al. 2013). The genome of $Z$. tritici is considered to be complete, i.e. without any gaps, and includes telomeres and centromeres (Goodwin et al. 2011), which should facilitate effector gene discovery (Thomma et al. 2016). However, thus far there are no known effector genes that are located on $Z$. tritici CDCs, and thus the role of these CDCs in pathogenicity remains poorly understood.

Comparative genomics of $Z$. tritici identified genomic compartments that harbour candidate effector genes. Comparative genomics between $Z$. tritici reference isolate IPO323 and the highly virulent isolate 3D7 identified orphan genomic regions that are unique to one of the two genomes (Plissonneau et al. 2016). The orphan regions in $Z$. tritici isolate 3D7 contain genes encoding secreted proteins (Plissonneau et al. 2016). Additionally, a recombination map was generated for $Z$. tritici to identify recombination hotspots, i.e. regions that display a high recombination frequency. Notably, these recombination hotspots are located in proximity to telomeres, and are enriched for genes encoding secreted proteins (Croll et al. 2015).

Genomes can be mined for homologs of effectors with known functions, as it can be anticipated that these homologs play similar roles in other fungal plant pathogens. Analysis of the $Z$. tritici genome indicated the presence of three effector genes encoding LysM proteins (Mg3LysM, Mg1LysM, and MgxLysM) (Marshall et al. 2011), which are homologs of Ecp6 (for extracellular protein 6), a LysM effector gene of the fungal tomato pathogen Cladosporium fulvum (de Jonge et al. 2010). Two of these effectors (Mg1LysM and Mg3LysM) have been functionally analysed, and both effectors are able to bind chitin and protect fungal hyphae against plant hydrolytic enzymes (Marshall et al. 2011). In addition, Mg3LysM is able to block the activation of chitin-induced immunity in host plants (Marshall et al. 2011).

Z. tritici genome and transcriptome analysis revealed many additional effector candidates that may be involved in virulence (Goodwin et al. 2011; Grandaubert et al. 2015; Rudd et al. 2015). For example, a genome-wide mining for genes encoding small, secreted, cysteine-rich proteins that are induced in planta, commonly used criteria to define effectors in fungal pathogens (Gibriel et al. 2016), revealed 78 effector candidates (Mirzadi Gohari et al. 2015). However, none of them have been functionally characterized as effector yet, by showing either a role in immune suppression or a contribution to virulence (Mirzadi Gohari et al. 2015). Additionally, three Z. tritici genes were found to be subject to positive selection and to play a role in virulence (Poppe et al. 2015). However, only one of them encodes a signal peptide and thus qualifies as a bona fide effector (Poppe et al. 2015).

In order to identify full effector repertoires of plant pathogens, it is required to have properly annotated gene models (Gibriel et al. 2016). However, for Z. tritici, many of 
the previously annotated gene models have incorrect exon/intron boundaries and lack either a start or a stop codon (Grandaubert et al. 2015). Arguably, such incorrect gene annotation compromises discovery of the complete effector catalog in this fungal pathogen.

\section{VERTICILLIUM WILT DISEASES}

It is unknown how broad host range pathogens engage in arms races with multiple hosts (Dong et al. 2015). Here, I focus on V. dahliae, a broad host range pathogen, to understand processes of host adaptation. This pathogen belongs to the genus Verticillium that comprises ten species of soil-borne ascomycete fungi (Inderbitzin et al. 2011). Few of these species collectively cause Verticillium wilt diseases on hundreds of plant hosts across the world (Inderbitzin et al. 2011; Klimes et al. 2015). For example, $V$. dahliae, which is the most notorious plant pathogenic species within this genus, is able to infect more than 200 plant hosts including economically important crops such as tomato, potato, strawberry, lettuce and cotton (Fradin and Thomma 2006; Inderbitzin et al. 2011). This pathogen infects the plant by penetrating the root, after which it enters the xylem and starts to produce conidia that are carried upward with the water flow to distal plant parts (Fradin and Thomma 2006). In contrast to $V$. dahliae, the other pathogenic Verticillium species ( $V$. albo-atrum, $V$. alfalfae, $V$. non-alfalfae, and $V$. longisporum) have considerably narrower host ranges.

Control of Verticillium wilt diseases is difficult due to the long viability of its resting structures and the inability of fungicides to affect the pathogens once they enter the xylem (Fradin and Thomma 2006). Therefore, the use of resistant cultivars is the preferred strategy for disease management. Thus far, tomato Ve1 is the only immune receptor that provides resistance against particular $V$. dahliae and $V$. alfalfae strains (Fradin et al. 2009). Recently, functional homologs of tomato Ve1 have also been identified in tobacco, potato, wild eggplant and hop (Song et al. 2016).

\section{Evolution of virulence in V. dahliae}

Sexual reproduction is considered an important driver for adaptive genome evolution. However, sexual reproduction has never been observed in $V$. dahliae although it is a successful pathogen that evolved the capacity to infect hundreds of hosts (Fradin and Thomma 2006; Inderbitzin and Subbarao 2014). Previously, genomic studies have provided insights into the evolution of virulence of $V$. dahliae. Genome comparisons of multiple $V$. dahliae strains revealed chromosomal length polymorphisms between closely related $V$. dahliae strains (de Jonge et al. 2013). These length polymorphisms are caused by extensive genome rearrangements that are mediated by erroneous doublestrand break repair pathways, often utilizing abundant transposable elements as a substrate for repair (Faino et al. 2016). Moreover, these chromosomal rearrangements facilitate the formation of highly dynamic, repeat-rich, lineage-specific (LS) regions that 
are only present in a subset of $V$. dahliae strains (de Jonge et al. 2013; Faino et al. 2016). Notably, these LS regions can amount to up to $4 \mathrm{Mb}$ of the total genome size and contain up to 1,000 genes (de Jonge et al. 2013). Interestingly, many in plantainduced effector genes are localized within these highly dynamic LS regions (de Jonge et al. 2013). For example, Ave1, which is an important effector gene that is involved in virulence on many host plants, was found to be localized within an LS region (de Jonge et al. 2012). Similarly, the gene encoding the LysM domain-containing Vd2LysM effector that is involved in virulence on tomato is located in an LS region (Kombrink et al. 2017). By definition, LS regions differ significantly between $V$. dahliae strains, and thus different $V$. dahliae strains commonly carry distinct sets of effector genes in their LS regions (de Jonge et al. 2013). However, $V$. dahliae strains with differences in their effector gene repertoires have the ability to infect the same host plant. For example, $V$. dahliae strain JR2 carries the Ave1 effector gene, which is involved in virulence in tomato plants lacking Ve1, while V. dahliae strain VdLs17 lacks this gene. Loss of this gene in VdLs17 reduces its virulence on tomato, although VdLs17 remains the capacity to infect this host plant (de Jonge et al. 2012). This $V$. dahliae strain uses other effectors than Ave1, such as Vd2LysM, to infect this host (Kombrink et al. 2017). Therefore, different LS effectors enable $V$. dahliae strains to infect the same host.

\section{MAIN RESEARCH QUESTION}

In this thesis research, I have used comparative population genomics to analyse the ascomycete plant pathogens $Z$. tritici and $V$. dahliae. My aim was to mine for genomic characteristics of these two pathogens that can explain their narrow or broad host range, respectively.

\section{THESIS OUTLINE}

In Chapter 2, I review recent advances in genome sequencing technologies, genome assembly, gene annotation, as well as effector identification methods to reveal complete effector repertoires. Finally, I explain my view of how the knowledge of effector diversity can be exploited to develop sustainable resistance breeding strategies.

In Chapter 3, I show that $V$. dahliae strains that are able to infect the same host plant harbour highly divergent LS effector repertoires. First, I determined effector genes localized within the core and lineage-specific (LS) genomic regions of a collection of $V$. dahliae strains. Subsequently, I determined the ability of these strains to infect a collection of plant species that belong to various plant families.

In Chapter 4, I show that $V$. dahliae strain 85S that infect sunflower plant harbour a hostspecific effector that contribute to disease establishment. First, I conducted comparative 
genomics of a sunflower pathogenic strain with a collection of non-pathogenic strains and identified an effector gene that exclusively occur in the sunflower pathogenic strain and that is highly induced during host colonization. Functional analysis revealed that this effector gene quantitatively contributes to $V$. dahliae virulence on sunflower, but not on other host plants.

In Chapter 5, I show extensive genetic diversity amongst Z. tritici isolates that are adapted to infect either bread wheat (BW) or durum wheat (DW). First, I conducted whole-genome sequencing of a worldwide collection of 136 Z. tritici isolates and phenotyping assays on a set of BW and DW cultivars. Subsequently, I assessed genomewide differences between BW and DW isolates and identified four effector genes carrying non-synonymous single nucleotide polymorphisms in BW isolates.

In Chapter 6, I show the cloning of the first avirulence effector gene of Z. tritici; AvrStb6, and show that this effector gene is maintained in $Z$. tritici populations. I first conducted comparative population genomics combined with genetic mapping and identified a polymorphic genomic region that harbours AvrStb6. The paradigm states that deployment of Stb6 leads to removal of this avirulence effector gene from pathogen populations. However, when we crossed Z. tritici on wheat, we found that sex occurs even with an avirulent parent, thus leading to the maintenance of AvrStb6 in subsequent populations.

In Chapter 7, the major findings of this thesis are discussed in a broader context and directions for future research are suggested. 


\section{REFERENCES}

Brown, J. K. M., Chartrain, L., Lasserre-Zuber, P., and Saintenac, C. 2015. Genetics of resistance to Zymoseptoria tritici and applications to wheat breeding. Fungal Genetics and Biology 79:33-41.

Chartrain, L., Brading, P. A., and Brown, J. K. M. 2005. Presence of the Stb6 gene for resistance to septoria tritici blotch (Mycosphaerella graminicola) in cultivars used in wheat-breeding programmes worldwide. Plant Pathology 54:134-143.

Cook, D. E., Mesarich, C. H., and Thomma, B. P. H. J. 2015. Understanding plant immunity as a surveillance system to detect invasion. Annual Review of Phytopathology 53:541-563.

Cools, H. J., and Fraaije, B. A. 2008. Are azole fungicides losing ground against Septoria wheat disease? Resistance mechanisms in Mycosphaerella graminicola. Pest Management Science 64:681-684.

Croll, D., Zala, M., and McDonald, B. A. 2013. Breakage-fusion-bridge cycles and large insertions contribute to the rapid evolution of accessory chromosomes in a fungal pathogen. PLoS Genetics 9:e1003567.

Croll, D., Lendenmann, M. H., Stewart, E., and McDonald, B. A. 2015. The impact of recombination hotspots on genome evolution of a fungal plant pathogen. Genetics 201:1213-1228.

de Jonge, R., Bolton, M. D., Kombrink, A., van den Berg, G. C., Yadeta, K. A., and Thomma, B. P. 2013. Extensive chromosomal reshuffling drives evolution of virulence in an asexual pathogen. Genome Research 23:1271-1282.

de Jonge, R., Peter van Esse, H., Kombrink, A., Shinya, T., Desaki, Y., Bours, R., van der Krol, S., Shibuya, N., Joosten, M. H. A. J., and Thomma, B. P. H. J. 2010. Conserved fungal LysM effector Ecp6 prevents chitin-triggered immunity in plants. Science 329:953-955.

de Jonge, R., Peter van Esse, H., Maruthachalam, K., Bolton, M. D., Santhanam, P., Saber, M. K., Zhang, Z., Usami, T., Lievens, B., and Subbarao, K. V. 2012. Tomato immune receptor Ve1 recognizes effector of multiple fungal pathogens uncovered by genome and RNA sequencing. Proceedings of the National Academy of Sciences of the United States of America 109:964-975.

Dong, S., Raffaele, S., and Kamoun, S. 2015. The two-speed genomes of filamentous pathogens: waltz with plants. Current Opinion in Genetics \& Development 35:5765.

Faino, L., Seidl, M. F., Shi-Kunne, X., Pauper, M., van den Berg, G. C. M., Wittenberg, A. H. J., and Thomma, B. P. H. J. 2016. Transposons passively and actively contribute to evolution of the two-speed genome of a fungal pathogen. Genome Research 26:1091-1100.

Fisher, M. C., Henk, D. A., Briggs, C. J., Brownstein, J. S., Madoff, L. C., McCraw, S. L., and Gurr, S. J. 2012. Emerging fungal threats to animal, plant and ecosystem health. Nature 484:186-194. 
Fones, H., and Gurr, S. 2015. The impact of Septoria tritici Blotch disease on wheat: An EU perspective. Fungal Genetics and Biology 79:3-7.

Fradin, E. F., and Thomma, B. P. H. J. 2006. Physiology and molecular aspects of Verticillium wilt diseases caused by $V$. dahliae and $V$. albo-atrum. Molecular Plant Pathology 7:71-86.

Fradin, E. F., Zhang, Z., Juarez Ayala, J. C., Castroverde, C. D., Nazar, R. N., Robb, J., Liu, C. M., and Thomma, B. P. 2009. Genetic dissection of Verticillium wilt resistance mediated by tomato Ve1. Plant Physiology 150:320-332.

Gibriel, H. A. Y., Thomma, B. P. H. J., and Seidl, M. F. 2016. The age of effectors: genomebased discovery and applications. Phytopathology 106:1206-1212.

Goodwin, S. B., Ben M’Barek, S., Dhillon, B., Wittenberg, A. H. J., Crane, C. F., Hane, J. K., Foster, A. J., Van der Lee, T. A. J., Grimwood, J., Aerts, A., Antoniw, J., Bailey, A., Bluhm, B., Bowler, J., Bristow, J., van der Burgt, A., Canto-CanchÈ, B., Churchill, A. C. L., Conde-Ferrłez, L., Cools, H. J., Coutinho, P. M., Csukai, M., Dehal, P., De Wit, P., Donzelli, B., van de Geest, H. C., van Ham, R. C. H. J., Hammond-Kosack, K. E., Henrissat, B., Kilian, A., Kobayashi, A. K., Koopmann, E., Kourmpetis, Y., Kuzniar, A., Lindquist, E., Lombard, V., Maliepaard, C., Martins, N., Mehrabi, R., Nap, J. P. H., Ponomarenko, A., Rudd, J. J., Salamov, A., Schmutz, J., Schouten, H. J., Shapiro, H., Stergiopoulos, I., Torriani, S. F. F., Tu, H., de Vries, R. P., Waalwijk, C., Ware, S. B., Wiebenga, A., Zwiers, L.-H., Oliver, R. P., Grigoriev, I. V., and Kema, G. H. J. 2011. Finished genome of the fungal wheat pathogen Mycosphaerella graminicola reveals dispensome structure, chromosome plasticity, and stealth pathogenesis. PLoS Genetics 7:e1002070.

Grandaubert, J., Bhattacharyya, A., and Stukenbrock, E. H. 2015. RNA-seq-Based gene annotation and comparative genomics of four fungal grass pathogens in the genus Zymoseptoria identify novel orphan genes and species-specific invasions of transposable elements. G3 5:1323-1333.

Hunter, T., Coker, RR., and Royle, DJ. 1999. The teleomorph stage, Mycosphaerella graminicola, in epidemics of septoria tritici blotch on winter wheat in the UK. Plant Pathology 48:51-57.

Inderbitzin, P., and Subbarao, K. V. 2014. Verticillium systematics and evolution: how confusion impedes Verticillium wilt management and how to resolve it. Phytopathology 104:564-574.

Inderbitzin, P., Bostock, R. M., Davis, R. M., Usami, T., Platt, H. W., and Subbarao, K. V. 2011. Phylogenetics and taxonomy of the fungal vascular wilt pathogen Verticillium, with the descriptions of five New species. PLoS One 6:e28341.

Jones, J. D. G., and Dangl, J. L. 2006. The plant immune system. Nature 444:323-329.

Kema, G. H. J., Annone, J. G., Sayoud, R., Van Silfhout, C. H., Van Ginkel, M., and De Bree, J. 1996. Genetic variation for virulence and resistance in the wheat-Mycosphaerella graminicola pathosystem. I. Interactions between pathogen isolates and host cultivars. Phytopathology 86, 200- 212. 
Kema, G. H. J., and van Silfhout, C. H. 1997. Genetic variation for virulence and resistance in the wheat-Mycosphaerella graminicola pathosystem III. Comparative seedling and adult plant experiments. Phytopathology 87:266-272.

Kettles, G. J., and Kanyuka, K. 2016. Dissecting the molecular interactions between wheat and the fungal pathogen Zymoseptoria tritici. Frontiers in Plant Science 7: 508.

Klimes, A., Dobinson, K. F., Thomma, B. P., and Klosterman, S. J. 2015. Genomics spurs rapid advances in our understanding of the biology of vascular wilt pathogens in the genus Verticillium. Annual Reviews of Phytopathology 53:181-198.

Kombrink, A., Rovenich, H., Shi-Kunne, X., Rojas-Padilla, E., van den Berg, G. C. M., Domazakis, E., de Jonge, R., Valkenburg, D.-J., Sánchez-Vallet, A., Seidl, M. F., and Thomma, B. P. H. J. 2017. Verticillium dahliae LysM effectors differentially contribute to virulence on plant hosts. Molecular Plant Pathology. 18:596-608.

Marshall, R., Kombrink, A., Motteram, J., Loza-Reyes, E., Lucas, J., Hammond-Kosack, K. E., Thomma, B. P. H. J., and Rudd, J. J. 2011. Analysis of two in planta expressed LysM effector homologs from the fungus Mycosphaerella graminicola reveals novel functional properties and varying contributions to virulence on wheat. Plant Physiology 156:756-769.

Metzker, M. L. 2010. Sequencing technologies - the next generation. Nature Reviews Genetics 11:31-46.

Mirzadi Gohari, A., Ware, S. B., Wittenberg, A. H. J., Mehrabi, R., Ben M'Barek, S., Verstappen, E. C. P., van der Lee, T. A. J., Robert, O., Schouten, H. J., de Wit, P. P. J. G. M., and Kema, G. H. J. 2015. Effector discovery in the fungal wheat pathogen Zymoseptoria tritici. Molecular Plant Pathology 16:931-945.

Plissonneau, C., Stürchler, A., and Croll, D. 2016. The evolution of orphan regions in genomes of a fungal pathogen of wheat. mBio 7: e01231-16.

Ponomarenko, A., Goodwin, S. B., and Kema, G. H. 2011. Septoria tritici blotch (STB) of wheat. Plant Health Instructor: 1-7.

Poppe, S., Dorsheimer, L., Happel, P., and Stukenbrock, E. H. 2015. Rapidly evolving genes are key players in host specialization and virulence of the fungal wheat pathogen Zymoseptoria tritici (Mycosphaerella graminicola). PLoS Pathogens 11:e1005055.

Raffaele, S., and Kamoun, S. 2012. Genome evolution in filamentous plant pathogens: why bigger can be better. Nature Reviews Microbiology 10:417-430.

Rovenich, H., Boshoven, J. C., and Thomma, B. P. H. J. 2014. Filamentous pathogen effector functions: of pathogens, hosts and microbiomes. Current Opinion in Plant Biology 20:96-103.

Rudd, J. J., Kanyuka, K., Hassani-Pak, K., Derbyshire, M., Andongabo, A., Devonshire, J., Lysenko, A., Saqi, M., Desai, N. M., Powers, S. J., Hooper, J., Ambroso, L., Bharti, A., Farmer, A., Hammond-Kosack, K. E., Dietrich, R. A., and Courbot, M. 2015. Transcriptome and metabolite profiling of the infection cycle of Zymoseptoria tritici on wheat reveals a biphasic interaction with plant immunity involving differential pathogen chromosomal contributions and a variation on the hemibiotrophic 
lifestyle definition. Plant Physiology 167:1158-1185.

Saintenac, C., Lee, W.-S., Cambon, F., Rudd, J. J., King, R. C., Marande, W., Powers, S. J., Bergès, H., Phillips, A. L., Uauy, C., Hammond-Kosack, K. E., Langin, T., and Kanyuka, K. 2018. Wheat receptor-kinase-like protein Stb6 controls gene-for-gene resistance to fungal pathogen Zymoseptoria tritici. Nature Genetics. 51:291.

Seidl, M. F., and Thomma, B. P. H. J. 2014. Sex or no sex: evolutionary adaptation occurs regardless. BioEssays 36:335-345.

Shiferaw, B., Smale, M., Braun, H.-J., Duveiller, E., Reynolds, M., and Muricho, G. 2013. Crops that feed the world 10 . Past successes and future challenges to the role played by wheat in global food security. Food Security 5:291-317.

Song, Y., Zhang, Z., Seidl, M. F., Majer, A., Jakse, J., Javornik, B., and Thomma, B. P. H. J. 2016. Broad taxonomic characterization of Verticillium wilt resistance genes reveals ancient origin of the tomato Ve1 immune receptor. Molecular Plant Pathology 19:77-89.

Stukenbrock, E. H., Jørgensen, F. G., Zala, M., Hansen, T. T., McDonald, B. A., and Schierup, M. H. 2010. Whole-genome and chromosome evolution associated with host adaptation and speciation of the wheat pathogen Mycosphaerella graminicola. PLoS Genetics 6:e1001189.

Thomma, B. P. H. J., Seidl, M. F., Shi-Kunne, X., Cook, D. E., Bolton, M. D., van Kan, J. A. L., and Faino, L. 2016. Mind the gap; seven reasons to close fragmented genome assemblies. Fungal Genetics and Biology 90:24-30.

Torriani, S. F., Melichar, J. P., Mills, C., Pain, N., Sierotzki, H., and Courbot, M. 2015. Zymoseptoria tritici: A major threat to wheat production, integrated approaches to control. Fungal Genetics Biology 79:8-12.

Wittenberg, A. H. J., van der Lee, T. A. J., Ben M'Barek, S., Ware, S. B., Goodwin, S. B., Kilian, A., Visser, R. G. F., Kema, G. H. J., and Schouten, H. J. 2009. Meiosis drives extraordinary genome plasticity in the haploid fungal plant pathogen Mycosphaerella graminicola. PLoS One 4:e5863. 


\section{Chapter 2}

$$
\text { do }
$$

$$
26
$$

ᄂ 


\section{The age of effectors: genome-based discovery and applications}

Hesham A.Y. Gibriel, Bart P.H.J. Thomma, Michael F. Seidl

Laboratory of Phytopathology, Wageningen University, Droevendaalsesteeg 1, 6708 PB Wageningen, The Netherlands.

Published in Phytopathology 106:1206-1212 (2016) 


\begin{abstract}
Microbial pathogens cause devastating diseases on economically and ecologically important plant species, threatening global food security, and causing billions of dollars of losses annually. During the infection process, pathogens secrete so-called effectors that support host colonization, often by deregulating host immune responses. Over the last decades, much of the research on molecular plant-microbe interactions has focused on the identification and functional characterization of such effectors. The increasing availability of sequenced plant pathogen genomes has enabled genomicsbased discovery of effector candidates. Nevertheless, identification of full plant pathogen effector repertoires is often hampered by erroneous gene annotation and the localization of effector genes in genomic regions that are notoriously difficult to assemble. Here, we argue that recent advances in genome sequencing technologies, genome assembly, gene annotation, as well as effector identification methods hold promise to disclose complete and correct effector repertoires. This allows to exploit complete effector repertoires, and knowledge of their diversity within pathogen populations, to develop durable and sustainable resistance breeding strategies, disease control and management of plant pathogens.
\end{abstract}




\section{INTRODUCTION}

Plant-associated microbes, which include bacteria, fungi, oomycetes and nematodes, play important roles in agriculture and in natural ecosystems. Some of these microbes cause devastating diseases that lead to $>40 \%$ yield losses on major crops (Fisher et al. 2012; Pennisi 2010), and therefore threaten global food security. To contain diseases caused by plant pathogens, farmers rely on multiple strategies including adaptation of cultural practices such as crop rotation, but also deployment of resistant crop varieties and the use of chemical control agents such as fungicides. Nevertheless, pathogens may evolve to rapidly overcome such control measures (McDonald and Linde 2002; Stukenbrock and McDonald 2008).

To establish a successful infection, plant pathogens need to overcome the plant immune system. Plant cells deploy extracellular and intracellular receptors to detect invasion patterns that indicate the presence or activity of pathogens to subsequently mount immune responses (Cook et al. 2015). In turn, pathogens secrete so-called effector proteins to deregulate these host responses (Cook et al. 2015; Rovenich et al. 2014). However, effectors themselves can become invasion patterns when they become recognized by plant immune receptors that trigger an immune response, leading to an incompatible interaction between the pathogen and the host (Cook et al. 2015). To avoid recognition by the plant immune system, pathogens in turn evolve novel effectors or modify or lose existing ones.

Plant pathogen genomes generally encode a plethora of effectors. Effector diversification is mediated by genomic mechanisms that range from single nucleotide polymorphisms to structural variations that can affect chromosomal shape and gene content (Dong et al. 2015; Raffaele and Kamoun 2012; Seidl and Thomma 2014). In the past, various methods have been used to identify effector repertoires, and to determine their diversity. Here, we discuss the recent progress in genomic-based methods for effector discovery, while focusing on effectors from plant pathogens with a biotrophic or hemibiotrophic lifestyle. Furthermore, we highlight how effector discovery can contribute to the development of resistance breeding strategies and disease management.

\section{GENOMICS-BASED EFFECTOR DISCOVERY}

More than a decade ago, the genome of the first plant associated microbe, the plant pathogenic bacterium Xylella fastidiosa, was sequenced (Simpson et al. 2000). Five years later, the rice blast fungus Magnaporthe oryzae was the first eukaryotic plant pathogen for which a genome sequence became available (Dean et al. 2005), soon followed by the genome sequences of two oomycete plant pathogens; Phytophthora sojae and $P$. ramorum (Tyler et al. 2006). All these genomes were sequenced using Sanger sequencing, which is laborious, costly, and low in throughput (Kircher and Kelso 
2010). Subsequent advances in high throughput sequencing technologies, termed next-generation sequencing (NGS), have allowed rapid sequencing of entire genomes at significantly reduced costs (Metzker 2010). This development also brought genomic sciences within reach of research laboratories that study non-model organisms. Consequently, the number of available genome sequences has increased exponentially. Whereas researchers were fortunate to have access to a genome sequence of their organism of interest a decade ago, by now the availability of genome sequences for a multitude of genotypes of the same species is becoming the standard. Phytopathologists are especially interested in population genomic data which allows to determine core and lineage-specific effector repertoires of their pathogen of interest, allowing to investigate effector dynamics and evolution. However, reliable assessment of effector repertoires remains a considerable challenge.

Pathogens secrete effectors that exert their activity either in the host's extracellular environment (apoplastic effectors) or inside host cells (cytoplasmic effectors) (Giraldo and Valent 2013). Effectors are generally secreted via the endoplasmic reticulum ER/ Golgi route, which requires a hydrophobic N-terminal signal peptide (von Heijne 1990). To date, most experimentally verified fungal and oomycete effectors contain such an N-terminal secretion signal (Lo Presti et al. 2015; Stergiopoulos and de Wit 2009). Therefore, genome-wide studies aiming to identify effector candidates commonly first predict the secretome, i.e. the repertoire of all secreted proteins, by querying for the presence of N-terminal signal peptides (Lo Presti et al. 2015).

Upon secretion into the extracellular space, effectors may subsequently be translocated into the host cytoplasm. In oomycetes, so far two distinct classes of cytoplasmic effectors have been described, the RXLRs and the Crinklers (CRNs). Both contain conserved amino acid motifs in the $\mathrm{N}$-terminal region downstream of the signal peptide, which have been proposed to facilitate host-cell translocation (Schornack et al. 2010; Whisson et al. 2007). RXLR effectors contain an RXLR motif that is often followed by a (D)EER motif, while CRNs contain an LXLFLAK motif (Haas et al. 2009; Torto et al. 2003; Whisson et al. 2007). The presence of these motifs can be identified using computational methods (Haas et al. 2009; Jiang et al. 2008).

Most fungal and oomycete effectors that have been functionally characterized to date are smaller than 300 amino acids with four or more cysteine residues (Stergiopoulos et al. 2013; Stergiopoulos and de Wit 2009). Importantly, however, these characteristics cannot be used as criteria to identify effectors, as also well-characterized effectors often lack these properties (Lo Presti et al. 2015; Sperschneider et al. 2015). Therefore, to exhaustingly describe effector repertoires of plant pathogens, universal features of effector candidates, which are their secretion and differential expression in planta, should be considered (Sperschneider et al. 2015). Automatic classification methods that do not solely rely on strict rules can rank proteins according to their likelihood of being effectors by integrating multiple effector properties, e.g. protein size and cysteine 
content, and thus can further aid effector identification (Saunders et al. 2012). Recently, 'EffectorP' has been introduced which is the first program that utilizes machine learning to improve the prediction of fungal effector proteins from secretomes (Sperschneider et al. 2016). 'EffectorP' uses sequence-derived features to predict fungal effectors from secretomes (Sperschneider et al. 2016), thereby limiting the direct dependency on the above mentioned rules of effector selection. However, fungal effectors are a very heterogeneous group of proteins and, even though novel approaches such as 'EffectorP' hold promises in improving effector discovery, effector proteins remain notoriously difficult to predict.

\section{Comparative genomics-based effector discovery}

The availability of genome sequences of closely related pathogens, or of different strains of the same pathogenic species, permit comparative studies to identify effectors and to determine core and lineage-specific effector repertoires of the pathogen of interest, allowing to investigate effector dynamics and evolution. Genome comparisons of the closely related fungal maize pathogens Ustilago maydis and Sporisorium reilianum revealed 43 genomic regions with decreased sequence conservation, some of which were completely absent in one of the species (Schirawski et al. 2010). Notably, these divergent regions harbour in planta induced genes encoding secreted proteins, likely representing effectors (Kamper et al. 2006; Schirawski et al. 2010). Deletion analyses in these effector-rich regions confirmed their contribution to pathogen virulence (Kamper et al. 2006; Schirawski et al. 2010), thereby highlighting the merit of comparative genomics to flag genes as candidate effectors.

Comparisons between related pathogens can also provide insights into core and lineage-specific effector repertoires. For example, genome comparison of multiple strains of the vascular wilt pathogen Verticillium dahliae identified large lineage-specific genomic regions, which are regions that are only present in a subset of the $V$. dahliae population (de Jonge et al. 2013). These regions are enriched for secreted, in planta induced effector candidates that contribute to plant colonization (de Jonge et al. 2013). Genome comparisons of 65 geographically diverse strains of the cassava bacterial blight pathogen Xanthomonas axonopodis pv. manihotis revealed multiple lineage-specific effector candidates (Bart et al. 2012). Moreover, multiple effectors were found to be highly conserved and have been maintained throughout the world in all field samples (Bart et al. 2012).

Effector genes in plant pathogens such as Leptosphaeria maculans and Fusarium oxysporum can also be located on distinct chromosomes that are under specific conditions not required for growth, and therefore have been referred to as conditionally dispensable chromosomes (CDCs) (Balesdent et al. 2013; Ma et al. 2010). Comparisons between CDCs of related pathogens have been shown to facilitate effector discovery (Williams et al. 2016). For example, comparisons between CDCs from legume-infecting Fusarium oxysporum f. sp. medicaginis with CDCs of F. oxysporum f. sp. pisi and $F$. 
oxysporum f. sp. ciceris revealed small conserved genomic regions that contain in planta expressed genes encoding secreted proteins, which likely represent conserved effectors with roles in pathogenicity on legume hosts (Williams et al. 2016).

\section{COMPARATIVE POPULATION GENOMICS- AND TRANSCRIPTOMICS-BASED DISCOVERY OF AVIRULENCE EFFECTOR GENES}

Genome comparisons can also be utilized to identify effectors that are recognized by the plant immune system, so called avirulence effectors. Here, comparisons of avirulent and virulent strains can lead to the identification of single nucleotide polymorphisms (SNPS) in the protein-coding regions of effectors or reveal presence/absence polymorphisms, i.e. genomic regions that are exclusive to avirulent or virulent strains. For example, comparative genomics of multiple virulent and avirulent $V$. dahliae strains uncovered a 50-kb region encoding a single highly expressed effector gene that is present in avirulent strains but absent in virulent strains (de Jonge et al. 2012). This effector was subsequently experimentally shown to be the virulence factor Ave1 that is recognized by Ve1-carrying tomato plants (de Jonge et al. 2012). In the melon wilt fungus $F$. oxysporum f. sp. melonis, the effector AVRFOM2, which is recognized by melon Fom-2, was recently identified by comparative genomics of virulent and avirulent $F$. oxysporum f. sp. melonis strains (Schmidt et al. 2016). Similar to comparative analyses that exploit genome sequences, the sequencing and subsequent analyses of transcriptomes can lead to the identification of effectors. For example, combining in silico predictions with transcriptome comparisons of virulent and avirulent Cladosporium fulvum strains led to the identification of two polymorphic effector candidates, one of which was later confirmed to encode Avr5, which is recognized by the tomato immune receptor Cf5 (Mesarich et al. 2014).

\section{ERRONEOUS GENOME ASSEMBLY AND ANNOTATION HAMPER EFFECTOR DISCOVERY}

Nowadays, genome sequencing projects that utilize second-generation sequencing technologies generate millions of short $(<500 \mathrm{bp})$ sequence fragments, so-called reads, of relatively high quality (1 sequence error per $1 \mathrm{~kb}$ ) (Metzker 2010). However, the assembly of these reads into contiguous sequences, ideally chromosomes, is significantly hampered by highly similar repetitive sequences such as transposable elements (TEs). Sequencing reads that are shorter than repetitive sequences lead to their collapse, or to fragmented genome assemblies. Moreover, repetitive sequences in ascomycetes are often targeted by a genome-defense mechanism that induces specific C to T mutations, called repeat induced point mutations (RIP) (Selker 2002). RIP thereby locally elevates the AT content, which is a known bias for many second-generation technologies leading to low sequencing yields in these regions which further impact 
genome assemblies (Dohm et al. 2008). Notably, in many plant pathogens, effector genes reside in repeat-rich genomic sub-compartments where repeats facilitate rapid effector diversification by promoting structural variations (Dong et al. 2015; Raffaele and Kamoun 2012; Seidl and Thomma 2014). Moreover, effectors are often flanked by repetitive elements that can be targeted by RIP, which can lead to rapid effector gene diversification as for example demonstrated in L. maculans (Gout et al. 2007). Based on the limitations inherent to second-generation sequencing technologies, effectorrich regions are often not completely assembled, thereby hampering the complete and accurate identification of effector repertoires and their diversity (Figure 1A) (Thomma et al. 2016).

Advances in sequencing technologies have opened possibilities to overcome the limitations of short-read sequencing leading to significant improvement in genome assemblies. Third-generation sequencing technologies such as Single-Molecule RealTime (SMRT) and Nanopore sequencing generate long sequencing reads $(>1 \mathrm{~kb})$ that can span entire repetitive sequences and therefore enable more contiguous genome assemblies (Ashton et al. 2015; Kim et al. 2014; Powers et al. 2013; Shin et al. 2013). However, reads from third generation sequencing platforms display a relatively high error rate ( 15\%). To correct these sequencing errors, two alternative approaches are commonly applied. Hybrid error corrections such as implemented in Nanocorr (Goodwin et al. 2015) or PacBio Corrected Reads (PBcR) use high-quality short reads aligned to thirdgeneration long reads to correct for sequencing errors (Koren et al. 2012). Alternatively, errors in long reads can be faithfully corrected by consensus-based approaches such as provided by HGAP (Chin et al. 2013) or Nanocorrect (Loman et al. 2015), if sequencing is performed at considerable depth ( $\geq 50 \mathrm{X}$ genomic coverage) (Faino et al. 2015). The costs involved in third-generation sequencing, in particular for large genomes, make this technology not yet widely adopted. Therefore, with the expected advances in sequencing technologies, i.e. longer reads with lower error rate and reduction in costs, sequencing and assembly of larger and complex (repeat-rich) genomes will become feasible. Sequence contiguity can be further increased by the application of optical mapping, which is a technique that constructs high-resolution restriction maps from a single DNA molecule (Levy-Sakin and Ebenstein 2013). Subsequently, the optical map is aligned to an in silico restriction map of the genome assembly, thereby facilitating the placement of assembled sequences into contiguous regions. This technology has been successfully applied for the genome assemblies of multiple plant pathogens such as Botrytis cinerea, F. oxysporum, P. infestans and V. dahliae (Amselem et al. 2011; Haas et al. 2009; Klosterman et al. 2011; Ma et al. 2010). Recently, the combination of longread sequencing using SMRT sequencing technology and optical mapping yielded the complete and gapless genome assemblies of two V. dahliae strains (Faino et al. 2015). Significantly, comparison to previous genome assemblies revealed a 3-fold increase in repetitive elements ( $12 \%$ ) compared to previous estimate of $\sim 4 \%$ (de Jonge et al. 2013; Faino et al. 2015; Klosterman et al. 2011; Seidl et al. 2015), indicating that repeats in the previous genome assemblies have been under-represented. Therefore, complete 
genome assemblies mark a crucial step towards the optimal prediction of full effector repertoires in repeat-rich regions.

Apart from fragmented genome assemblies, incorrect gene annotation can hamper effector discovery (Figure 1B). Many of the previously annotated gene models in the fungal wheat pathogen $Z$. tritici have incorrect exon/intron boundaries and about 735 lack either start or stop codons (Gibriel et al. unpublished), which likely compromised the study of effectors candidates that had been selected based on these erroneous gene models (Mirzadi Gohari et al. 2015). Read data obtained from NGS-based RNA sequencing (RNA-seq) can be mapped to the reference genome, which provides a valuable guide for gene annotation as it reveals the exon-intron boundaries, and correct transcription start/stop sites (Figure 1B) (Yandell and Ence 2012). To achieve a high-quality gene prediction, $a b$ initio or alignment based gene predictions can be supplemented with RNA-seq data (Faino and Thomma 2014). There has been a steady progress in the suite of gene-prediction tools that combine RNA-seq data with other lines of evidence including Maker2 (Holt and Yandell 2011), EVidenceModeler (EVM) (Haas et al. 2008), and BRAKER1 (Hoff et al. 2015). By combining ab initio gene predictions with RNA-seq supported data, the previous gene annotation of $Z$. tritici was recently re-assessed, leading to the correction of 1,555 gene models (Grandaubert et al. 2015). Next to transcriptomics, also proteomics can be used to validate and correct gene annotation, in particular of those genes encoding secreted proteins. For example, a large-scale proteomic study aiming to profile the extracellular proteome of $P$. infestans validated the extracellular nature of 254 proteins previously predicted to be secreted (Meijer et al. 2014). Moreover, this analyses also led to the correction of about 150 open reading frames in the $P$. infestans genome which were previously incorrectly annotated (Meijer et al. 2014).

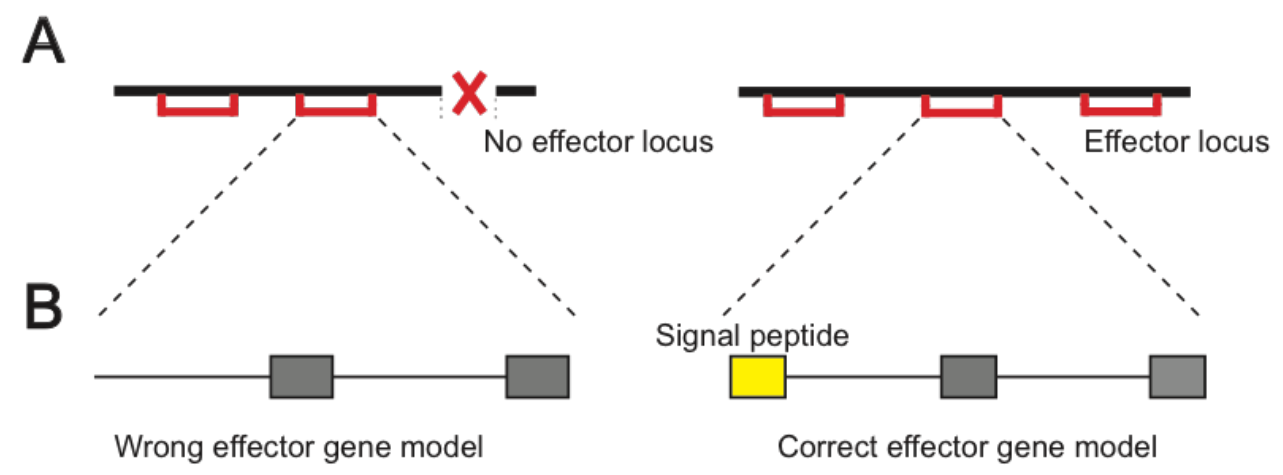

Figure 1. Erroneous genome assembly and annotation hamper effector discovery. Correct effector repertoires are obtained with proper assembly and annotation. (A) A stretch of DNA (black bar) that contains multiple effector genes (red blocks) is shown. In a fragmented genome assembly, an effector gene might be missing, as effectors are often located in 
repeat-rich regions. In gapless genome assemblies, all effector genes are present. (B) Identification of effector genes in the predicted gene set is influenced by the quality of gene annotation. Applying effector selection criteria will miss or wrongly classify effector candidate in erroneous gene models that e.g. miss the correct start/stop codon. Mining of correctly annotated gene models with correct start/stop codon and signal peptides will yield full effector repertoires.

\section{USING EFFECTORS AS A TOOL TO SCREEN FOR DISEASE RESISTANCE}

Effector repertoires determined by genomics-based discoveries can be exploited to aid breeding for disease resistance. 'Effectoromics' is a large-scale screening approach that uses effector candidates to identify host resistance $(R)$ genes (Vleeshouwers et al. 2008). This approach is based on transient expression of candidate effectors in plant leaves e.g. by agroinfiltration (Agrobacterium tumefaciens transient transformation assay) and/or a virus vector such as Potato virus X (PVX) (Vleeshouwers et al. 2008). Subsequently, plants are screened for the occurrence of hypersensitive cell death responses (HR), indicating the recognition of the effector by a matching plant immune receptor. 'Effectoromics' can contribute to resistance breeding by accelerating the identification of immune receptors when the matching effectors are available, as it replaces the slow process of generating stable transformants (Du and Vleeshouwers 2014; Vleeshouwers et al. 2008).

Effectors can target genes that critically facilitate compatible interactions between pathogens and their hosts, and thus these targets have often been referred to as susceptibility genes (van Schie and Takken 2014). Previously, susceptibility genes were identified by screening plant germplasm for recessive resistance (Bai et al. 2005), or by induced mutations that confer loss-of-susceptibility towards pathogens, which led to the identification of about 30 susceptibility genes (Gawehns et al. 2013). Alternatively, knowledge on the effector repertoire enables the usage of effectors as guides to identify plant susceptibility genes, for example by using protein-protein interaction assays such as yeast-two hybrid screening (Bos et al. 2010). Upon identification of a susceptibility gene, inactivation by subsequent mutations may confer disease resistance (Lewis et al. 2012), as this might interfere with the capability of the effectors to interact with their targets, and thereby limit the pathogen's ability to survive (Gawehns et al. 2013; van Schie and Takken 2014). Durability of resistance is a priority in plant breeding. One way to achieve durable resistance against plant pathogens is by the incorporation of multiple immune receptor genes into a single cultivar (gene pyramiding) as these can detect multiple effectors of the same pathogen (Michelmore et al. 2013). However, breeding multiple immune receptor genes into a cultivar is a time-consuming process and requires a sufficient number of previously identified receptor genes (Michelmore et al. 2013). Alternatively, susceptibility genes can provide more durable resistance than deployment of immune receptors, as they are essential for pathogen survival (van Schie 
and Takken 2014). However, mutations in susceptibility genes often have pleiotropic effects such as dwarfism or sensitivity to other stresses, thereby limiting their utilization in agriculture (Gawehns et al. 2013).

Knowledge on effectors can also be used to develop protection strategies against pathogens, as silencing these effectors might lead to reduced pathogen development. Host-induced gene silencing (HIGS) is based on exploiting an RNA silencing mechanism to target a selected pathogen gene, for example an effector gene, via the host plant (Nowara et al. 2010). Plants can be transformed with hairpin RNA constructs that target selected pathogen effectors to suppress their expression. For example, HIGS in barley expressing dsRNA targeting the effector gene Avra10 from the fungus Blumeria graminis led to reduced disease incidence (Nowara et al. 2010). To avoid pathogen evasion by loss of the targeted effector, conserved genes that are unlikely to be lost by the pathogen can be targeted. For example, HIGS in barley targeting the cytochrome P450 gene CYP51 of the wheat pathogen Fusarium graminearum leads to complete plant immunity (Koch et al. 2013). Even though HIGS has the potential to become a powerful tool for disease control, its application requires the usage of genetically modified plants, which is restricted under current legislative and public consent in many countries worldwide.

\section{MONITORING PATHOGEN FIELD POPULATIONS TO ASSESS EFFECTOR DIVERSITY}

DNA and RNA sequencing technologies allow to monitor pathogen populations in fields, and thereby enable pathogen surveillance. For example, field transcriptome sequencing of Puccinia striiformis f. sp. tritici (PST) infected wheat revealed a dramatic shift in the pathogen population structure in the UK (Hubbard et al. 2015). Similarly, genome and transcriptome analysis of different strains of $P$. infestans provided insights into the emergence of the aggressive lineage 13_A2 in northwest Europe (Cooke et al. 2012). Genome sequencing enables researchers to rapidly assess effector diversity, which can indicate the emergence of new pathogens that have the potential to overcome host resistance in the field (Figure 2). Therefore, monitoring of effector diversity, and subsequent deployment of plants carrying the matching resistance gene, would greatly contribute to disease management. The application of sequencing technologies to monitor pathogen migration and diversity will likely further accelerate in the future. Rapid, nearly real-time genome sequencing of pathogen populations can be applied on field isolates using the MinilON system, which is a portable sequencer that sequences individual DNA molecules using Nanopore technology. MinilON can presently sequence bacterial and viral genomes in as little as 48 hours (Check Hayden 2015; Quick et al. 2015; Quick et al. 2016). For example, MinION was used for real-time detection of Salmonella enterica from clinical isolates during a hospital outbreak, and yielded reliable clinical information in less than half a day (Quick et al. 2015). Similarly, during the 2015 Ebola 
virus outbreak in West Africa, real-time genomic sequencing revealed insights into virus diversity, and provided genomic information for epidemiological investigations to monitor disease dissemination (Quick et al. 2016). In the future, plant pathogen genomes can be rapidly sequenced with this device, disclosing information on their effector repertoires. Further fueled by technological advances of the MinION platform, we anticipate that real-time disease monitoring by genome sequencing in agricultural settings will provide unprecedented opportunities to monitor the spread of plant pathogens, and will be a prerequisite to install timely and suitable countermeasures.

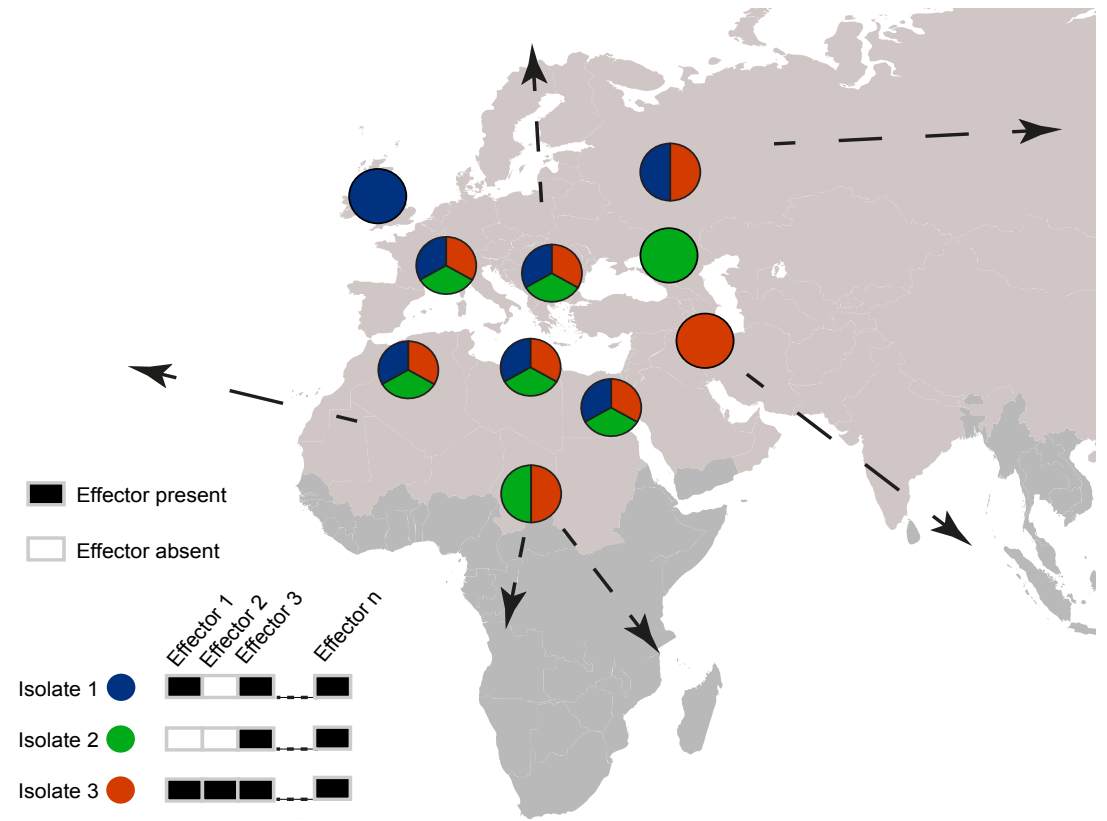

Figure 2. Monitoring of the spatial dispersal of plant pathogens. Rapid sequencing of plant pathogen genomes from field samples and subsequently identification of their effector repertories allows rapid insight into pathogen populations. Here, for example, a hypothetical pathogen population in several regions across Europe, Asia, and Africa, can be divided into three distinct lineages based on their effector repertoires, with the relative occurrence of each individual lineage within a geographic region shown by the pie chart. Sequencing of pathogen field samples and knowledge of their effector repertoires can be used to track the dispersal of pathogen lineages into new geographic regions (arrows).

\section{ACKNOWLEDGMENTS}

Work in the laboratory of BPHJT is support by the Research Council Earth and Life Sciences (ALW) of the Netherlands Organization of Scientific Research (NWO). MFS acknowledges the receipt of a VENI grant of ALW-NWO. 


\section{REFERENCES}

Amselem, J., Cuomo, C. A., van Kan, J. A. L., Viaud, M., Benito, E. P., Couloux, A., Coutinho, P. M., de Vries, R. P., Dyer, P. S., Fillinger, S., Fournier, E., Gout, L., Hahn, M., Kohn, L., Lapalu, N., Plummer, K. M., Pradier, J.-M., Quévillon, E., Sharon, A., Simon, A., ten Have, A., Tudzynski, B., Tudzynski, P., Wincker, P., Andrew, M., Anthouard, V., Beever, R. E., Beffa, R., Benoit, I., Bouzid, O., Brault, B., Chen, Z., Choquer, M., Collémare, J., Cotton, P., Danchin, E. G., Da Silva, C., Gautier, A., Giraud, C., Giraud, T., Gonzalez, C., Grossetete, S., Güldener, U., Henrissat, B., Howlett, B. J., Kodira, C., Kretschmer, M., Lappartient, A., Leroch, M., Levis, C., Mauceli, E., Neuvéglise, C., Oeser, B., Pearson, M., Poulain, J., Poussereau, N., Quesneville, H., Rascle, C., Schumacher, J., Ségurens, B., Sexton, A., Silva, E., Sirven, C., Soanes, D. M., Talbot, N. J., Templeton, M., Yandava, C., Yarden, O., Zeng, Q., Rollins, J. A., Lebrun, M.-H., and Dickman, M. 2011. Genomic analysis of the necrotrophic fungal pathogens Sclerotinia sclerotiorum and Botrytis cinerea. PLoS Genetics 7:e1002230.

Ashton, P. M., Nair, S., Dallman, T., Rubino, S., Rabsch, W., Mwaigwisya, S., Wain, J., and O'Grady, J. 2015. MinION nanopore sequencing identifies the position and structure of a bacterial antibiotic resistance island. Nature Biotechnology 33:296300.

Bai, Y., van der Hulst, R., Bonnema, G., Marcel, T. C., Meijer-Dekens, F., Niks, R. E., and Lindhout, P. 2005. Tomato defense to Oidium neolycopersici: dominant $\mathrm{Ol}$ genes confer isolate-dependent resistance via a different mechanism than recessive ol-2. Molecular Plant-Microbe Interaction 18:354-362.

Balesdent, M.-H., Fudal, I., Ollivier, B., Bally, P., Grandaubert, J., Eber, F., Chèvre, A.-M., Leflon, M., and Rouxel, T. 2013. The dispensable chromosome of Leptosphaeria maculans shelters an effector gene conferring avirulence towards Brassica rapa. New Phytologist 198:887-898.

Bart, R., Cohn, M., Kassen, A., McCallum, E. J., Shybut, M., Petriello, A., Krasileva, K., Dahlbeck, D., Medina, C., Alicai, T., Kumar, L., Moreira, L. M., Neto, J. R., Verdier, V., Santana, M. A., Kositcharoenkul, N., Vanderschuren, H., Gruissem, W., Bernal, A., and Staskawicz, B. J. 2012. High-throughput genomic sequencing of cassava bacterial blight strains identifies conserved effectors to target for durable resistance. Proceedings of the National Academy of Sciences of the United States of America 109:E1972-E1979.

Bos, J. I. B., Armstrong, M. R., Gilroy, E. M., Boevink, P. C., Hein, I., Taylor, R. M., Zhendong, T., Engelhardt, S., Vetukuri, R. R., Harrower, B., Dixelius, C., Bryan, G., Sadanandom, A., Whisson, S. C., Kamoun, S., and Birch, P. R. J. 2010. Phytophthora infestans effector AVR3a is essential for virulence and manipulates plant immunity by stabilizing host E3 ligase CMPG1. Proceedings of the National Academy of Sciences of the United States of America 107:9909-9914.

Check Hayden, E. 2015. Pint-sized DNA sequencer impresses first users. Nature 521:1516. 
Chin, C. S., Alexander, D. H., Marks, P., Klammer, A. A., Drake, J., Heiner, C., Clum, A., Copeland, A., Huddleston, J., Eichler, E. E., Turner, S. W., and Korlach, J. 2013. Nonhybrid, finished microbial genome assemblies from long-read SMRT sequencing data. Nature Methods 10:563-569.

Cook, D. E., Mesarich, C. H., and Thomma, B. P. H. J. 2015. Understanding plant immunity as a surveillance system to detect invasion. Annual Review of Phytopathology 53:541-563.

Cooke, D. E. L., Cano, L. M., Raffaele, S., Bain, R. A., Cooke, L. R., Etherington, G. J., Deahl, K. L., Farrer, R. A., Gilroy, E. M., Goss, E. M., Grünwald, N. J., Hein, I., MacLean, D., McNicol, J. W., Randall, E., Oliva, R. F., Pel, M. A., Shaw, D. S., Squires, J. N., Taylor, M. C., Vleeshouwers, V. G. A. A., Birch, P. R. J., Lees, A. K., and Kamoun, S. 2012. Genome analyses of an aggressive and invasive lineage of the Irish potato famine pathogen. PLoS Pathogens 8:e1002940.

de Jonge, R., Bolton, M. D., Kombrink, A., van den Berg, G. C. M., Yadeta, K. A., and Thomma, B. P. H. J. 2013. Extensive chromosomal reshuffling drives evolution of virulence in an asexual pathogen. Genome Research 23:1271-1282.

de Jonge, R., Peter van Esse, H., Maruthachalam, K., Bolton, M. D., Santhanam, P., Saber, M. K., Zhang, Z., Usami, T., Lievens, B., Subbarao, K. V., and Thomma, B. P. H. J. 2012. Tomato immune receptor Ve1 recognizes effector of multiple fungal pathogens uncovered by genome and RNA sequencing. Proceedings of the National Academy of Sciences of the United States of America 109:5110-5115.

Dean, R. A., Talbot, N. J., Ebbole, D. J., Farman, M. L., Mitchell, T. K., Orbach, M. J., Thon, M., Kulkarni, R., Xu, J.-R., Pan, H., Read, N. D., Lee, Y.-H., Carbone, I., Brown, D., Oh, Y. Y., Donofrio, N., Jeong, J. S., Soanes, D. M., Djonovic, S., Kolomiets, E., Rehmeyer, C., Li, W., Harding, M., Kim, S., Lebrun, M.-H., Bohnert, H., Coughlan, S., Butler, J., Calvo, S., Ma, L.-J., Nicol, R., Purcell, S., Nusbaum, C., Galagan, J. E., and Birren, B. W. 2005. The genome sequence of the rice blast fungus Magnaporthe grisea. Nature 434:980-986.

Dohm, J. C., Lottaz, C., Borodina, T., and Himmelbauer, H. 2008. Substantial biases in ultra-short read data sets from high-throughput DNA sequencing. Nucleic Acids Research 36:e105.

Dong, S., Raffaele, S., and Kamoun, S. 2015. The two-speed genomes of filamentous pathogens: waltz with plants. Current Opinion in Genetics \& Development 35:5765.

Du, J., and Vleeshouwers, V. G. 2014. The do's and don'ts of effectoromics. Methods Molecular Biology 1127:257-268.

Faino, L., and Thomma, B. P. H. J. 2014. Get your high-quality low-cost genome sequence. Trends in Plant Science 19:288-291.

Faino, L., Seidl, M. F., Datema, E., van den Berg, G. C. M., Janssen, A., Wittenberg, A. H. J., and Thomma, B. P. H. J. 2015. Single-molecule real-time sequencing combined with optical mapping yields completely finished fungal genome. mBio 6:e0093615. 
Fisher, M. C., Henk, D. A., Briggs, C. J., Brownstein, J. S., Madoff, L. C., McCraw, S. L., and Gurr, S. J. 2012. Emerging fungal threats to animal, plant and ecosystem health. Nature 484:186-194.

Gawehns, F., Cornelissen, B. J. C., and Takken, F. L. W. 2013. The potential of effectortarget genes in breeding for plant innate immunity. Microbial Biotechnology 6:223229.

Giraldo, M. C., and Valent, B. 2013. Filamentous plant pathogen effectors in action. Nature Review Microbiology 11:800-814.

Goodwin, S., Gurtowski, J., Ethe-Sayers, S., Deshpande, P., Schatz, M. C., and McCombie, W. R. 2015. Oxford Nanopore sequencing, hybrid error correction, and de novo assembly of a eukaryotic genome. Genome Research 25:1750-1756.

Gout, L., Kuhn, M. L., Vincenot, L., Bernard-Samain, S., Cattolico, L., Barbetti, M., Moreno-Rico, O., Balesdent, M.-H., and Rouxel, T. 2007. Genome structure impacts molecular evolution at the AvrLm1 avirulence locus of the plant pathogen Leptosphaeria maculans. Environmental Microbiology 9:2978-2992.

Grandaubert, J., Bhattacharyya, A., and Stukenbrock, E. H. 2015. RNA-seq-based gene annotation and comparative genomics of four fungal grass pathogens in the genus Zymoseptoria identify novel orphan genes and species-specific invasions of transposable elements. G3 5:1323-1333.

Haas, B. J., Salzberg, S. L., Zhu, W., Pertea, M., Allen, J. E., Orvis, J., White, O., Buell, C. R., and Wortman, J. R. 2008. Automated eukaryotic gene structure annotation using EVidenceModeler and the Program to Assemble Spliced Alignments. Genome Biology 9:1-22.

Haas, B. J., Kamoun, S., Zody, M. C., Jiang, R. H., Handsaker, R. E., Cano, L. M., Grabherr, M., Kodira, C. D., Raffaele, S., Torto-Alalibo, T., Bozkurt, T. O., Ah-Fong, A. M., Alvarado, L., Anderson, V. L., Armstrong, M. R., Avrova, A., Baxter, L., Beynon, J., Boevink, P. C., Bollmann, S. R., Bos, J. I., Bulone, V., Cai, G., Cakir, C., Carrington, J. C., Chawner, M., Conti, L., Costanzo, S., Ewan, R., Fahlgren, N., Fischbach, M. A., Fugelstad, J., Gilroy, E. M., Gnerre, S., Green, P. J., Grenville-Briggs, L. J., Griffith, J., Grunwald, N. J., Horn, K., Horner, N. R., Hu, C. H., Huitema, E., Jeong, D. H., Jones, A. M., Jones, J. D., Jones, R. W., Karlsson, E. K., Kunjeti, S. G., Lamour, K., Liu, Z., Ma, L., Maclean, D., Chibucos, M. C., McDonald, H., McWalters, J., Meijer, H. J., Morgan, W., Morris, P. F., Munro, C. A., O’Neill, K., Ospina-Giraldo, M., Pinzon, A., Pritchard, L., Ramsahoye, B., Ren, Q., Restrepo, S., Roy, S., Sadanandom, A., Savidor, A., Schornack, S., Schwartz, D. C., Schumann, U. D., Schwessinger, B., Seyer, L., Sharpe, T., Silvar, C., Song, J., Studholme, D. J., Sykes, S., Thines, M., van de Vondervoort, P. J., Phuntumart, V., Wawra, S., Weide, R., Win, J., Young, C., Zhou, S., Fry, W., Meyers, B. C., van West, P., Ristaino, J., Govers, F., Birch, P. R., Whisson, S. C., Judelson, H. S., and Nusbaum, C. 2009. Genome sequence and analysis of the Irish potato famine pathogen Phytophthora infestans. Nature 461:393-398.

Hoff, K. J., Lange, S., Lomsadze, A., Borodovsky, M., and Stanke, M. 2015. BRAKER1: Unsupervised RNA-Seq-Based Genome Annotation with GeneMark-ET and AUGUSTUS. Bioinformatics 32:767-769. 
Holt, C., and Yandell, M. 2011. MAKER2: an annotation pipeline and genome-database management tool for second-generation genome projects. BMC Bioinformatics 12:491.

Hubbard, A., Lewis, C., Yoshida, K., Ramirez-Gonzalez, R., de Vallavieille-Pope, C., Thomas, J., Kamoun, S., Bayles, R., Uauy, C., and Saunders, D. 2015. Field pathogenomics reveals the emergence of a diverse wheat yellow rust population. Genome Biology 16:23.

Jiang, R. H. Y., Tripathy, S., Govers, F., and Tyler, B. M. 2008. RXLR effector reservoir in two Phytophthora species is dominated by a single rapidly evolving superfamily with more than 700 members. Proceedings of the National Academy of Sciences of the United States of America 105:4874-4879.

Kamper, J., Kahmann, R., Bolker, M., Ma, L.-J., Brefort, T., Saville, B. J., Banuett, F., Kronstad, J. W., Gold, S. E., Muller, O., Perlin, M. H., Wosten, H. A. B., de Vries, R., Ruiz-Herrera, J., Reynaga-Pena, C. G., Snetselaar, K., McCann, M., Perez-Martin, J., Feldbrugge, M., Basse, C. W., Steinberg, G., Ibeas, J. I., Holloman, W., Guzman, P., Farman, M., Stajich, J. E., Sentandreu, R., Gonzalez-Prieto, J. M., Kennell, J. C., Molina, L., Schirawski, J., Mendoza-Mendoza, A., Greilinger, D., Munch, K., Rossel, N., Scherer, M., Vranes, M., Ladendorf, O., Vincon, V., Fuchs, U., Sandrock, B., Meng, S., Ho, E. C. H., Cahill, M. J., Boyce, K. J., Klose, J., Klosterman, S. J., Deelstra, H. J., Ortiz-Castellanos, L., Li, W., Sanchez-Alonso, P., Schreier, P. H., Hauser-Hahn, I., Vaupel, M., Koopmann, E., Friedrich, G., Voss, H., Schluter, T., Margolis, J., Platt, D., Swimmer, C., Gnirke, A., Chen, F., Vysotskaia, V., Mannhaupt, G., Guldener, U., Munsterkotter, M., Haase, D., Oesterheld, M., Mewes, H.-W., Mauceli, E. W., DeCaprio, D., Wade, C. M., Butler, J., Young, S., Jaffe, D. B., Calvo, S., Nusbaum, C., Galagan, J., and Birren, B. W. 2006. Insights from the genome of the biotrophic fungal plant pathogen Ustilago maydis. Nature 444:97-101.

Kim, K. E., Peluso, P., Babayan, P., Yeadon, P. J., Yu, C., Fisher, W. W., Chin, C.-S., Rapicavoli, N. A., Rank, D. R., Li, J., Catcheside, D. E. A., Celniker, S. E., Phillippy, A. M., Bergman, C. M., and Landolin, J. M. 2014. Long-read, whole-genome shotgun sequence data for five model organisms. Scientific Data 1:140045.

Kircher, M., and Kelso, J. 2010. High-throughput DNA sequencing - concepts and limitations. BioEssays 32:524-536.

Klosterman, S. J., Subbarao, K. V., Kang, S., Veronese, P., Gold, S. E., Thomma, B. P., Chen, Z., Henrissat, B., Lee, Y. H., Park, J., Garcia-Pedrajas, M. D., Barbara, D. J., Anchieta, A., de Jonge, R., Santhanam, P., Maruthachalam, K., Atallah, Z., Amyotte, S. G., Paz, Z., Inderbitzin, P., Hayes, R. J., Heiman, D. I., Young, S., Zeng, Q., Engels, R., Galagan, J., Cuomo, C. A., Dobinson, K. F., and Ma, L. J. 2011. Comparative genomics yields insights into niche adaptation of plant vascular wilt pathogens. PLoS Pathogens 7:e1002137.

Koch, A., Kumar, N., Weber, L., Keller, H., Imani, J., and Kogel, K.-H. 2013. Host-induced gene silencing of cytochrome P450 lanosterol C14 $\alpha$-demethylase-encoding genes confers strong resistance to Fusarium species. Proceedings of the National Academy of Sciences of the United States of America 110:19324-19329. 
Koren, S., Schatz, M. C., Walenz, B. P., Martin, J., Howard, J. T., Ganapathy, G., Wang, Z., Rasko, D. A., McCombie, W. R., Jarvis, E. D., and Phillippy, A. M. 2012. Hybrid error correction and de novo assembly of single-molecule sequencing reads. Nature Biotechnology 30:693-700.

Levy-Sakin, M., and Ebenstein, Y. 2013. Beyond sequencing: optical mapping of DNA in the age of nanotechnology and nanoscopy. Current Opinion in Biotechnology 24:690-698.

Lewis, J. D., Wan, J., Ford, R., Gong, Y., Fung, P., Nahal, H., Wang, P. W., Desveaux, D., and Guttman, D. S. 2012. Quantitative interactor screening with next-generation sequencing (QIS-Seq) identifies Arabidopsis thaliana MLO2 as a target of the Pseudomonas syringae type III effector HopZ2. BMC Genomics 13:8-8.

Lo Presti, L., Lanver, D., Schweizer, G., Tanaka, S., Liang, L., Tollot, M., Zuccaro, A., Reissmann, S., and Kahmann, R. 2015. Fungal effectors and plant susceptibility. Annual Review of Plant Biology 66:513-545.

Loman, N. J., Quick, J., and Simpson, J. T. 2015. A complete bacterial genome assembled de novo using only nanopore sequencing data. Nature Methods 12:733-735.

Ma, L.-J., van der Does, H. C., Borkovich, K. A., Coleman, J. J., Daboussi, M.-J., Di Pietro, A., Dufresne, M., Freitag, M., Grabherr, M., Henrissat, B., Houterman, P. M., Kang, S., Shim, W.-B., Woloshuk, C., Xie, X., Xu, J.-R., Antoniw, J., Baker, S. E., Bluhm, B. H., Breakspear, A., Brown, D. W., Butchko, R. A. E., Chapman, S., Coulson, R., Coutinho, P. M., Danchin, E. G. J., Diener, A., Gale, L. R., Gardiner, D. M., Goff, S., HammondKosack, K. E., Hilburn, K., Hua-Van, A., Jonkers, W., Kazan, K., Kodira, C. D., Koehrsen, M., Kumar, L., Lee, Y.-H., Li, L., Manners, J. M., Miranda-Saavedra, D., Mukherjee, M., Park, G., Park, J., Park, S.-Y., Proctor, R. H., Regev, A., Ruiz-Roldan, M. C., Sain, D., Sakthikumar, S., Sykes, S., Schwartz, D. C., Turgeon, B. G., Wapinski, I., Yoder, O., Young, S., Zeng, Q., Zhou, S., Galagan, J., Cuomo, C. A., Kistler, H. C., and Rep, M. 2010. Comparative genomics reveals mobile pathogenicity chromosomes in Fusarium. Nature 464:367-373.

McDonald, B. A., and Linde, C. 2002. Pathogen population genetics, evolutionary potential, and durable resistance. Annual Review of Phytopathology 40:349-379.

Meijer, H. J. G., Mancuso, F. M., Espadas, G., Seidl, M. F., Chiva, C., Govers, F., and Sabidó, E. 2014. Profiling the secretome and extracellular proteome of the potato late blight pathogen Phytophthora infestans. Molecular \& Cellular Proteomics 13:2101-2113.

Mesarich, C. H., Griffiths, S. A., van der Burgt, A., Ökmen, B., Beenen, H. G., Etalo, D. W., Joosten, M. H. A. J., and de Wit, P. J. G. M. 2014. Transcriptome sequencing uncovers the Avr5 avirulence gene of the tomato leaf mold pathogen Cladosporium fulvum. Molecular Plant-Microbe Interactions 27:846-857.

Metzker, M. L. 2010. Sequencing technologies - the next generation. Nature Review Genetics 11:31-46.

Michelmore, R. W., Christopoulou, M., and Caldwell, K. S. 2013. Impacts of resistance gene genetics, function, and evolution on a durable future. Annual Review of Phytopathology 51:291-319. 
Mirzadi Gohari, A., Ware, S. B., Wittenberg, A. H. J., Mehrabi, R., Ben M'Barek, S., Verstappen, E. C. P., van der Lee, T. A. J., Robert, O., Schouten, H. J., de Wit, P. P. J. G. M., and Kema, G. H. J. 2015. Effector discovery in the fungal wheat pathogen Zymoseptoria tritici. Molecular Plant Pathology 16:931-945.

Nowara, D., Gay, A., Lacomme, C., Shaw, J., Ridout, C., Douchkov, D., Hensel, G., Kumlehn, J., and Schweizer, P. 2010. HIGS: host-induced gene silencing in the obligate biotrophic fungal pathogen Blumeria graminis. The Plant Cell 22:3130-3141.

Pennisi, E. 2010. Armed and dangerous. Science 327:804-805.

Powers, J. G., Weigman, V. J., Shu, J., Pufky, J. M., Cox, D., and Hurban, P. 2013. Efficient and accurate whole genome assembly and methylome profiling of $E$. coli. BMC Genomics 14:1-18.

Quick, J., Ashton, P., Calus, S., Chatt, C., Gossain, S., Hawker, J., Nair, S., Neal, K., Nye, K., Peters, T., De Pinna, E., Robinson, E., Struthers, K., Webber, M., Catto, A., Dallman, T., Hawkey, P., and Loman, N. 2015. Rapid draft sequencing and real-time nanopore sequencing in a hospital outbreak of Salmonella. Genome Biology 16:114.

Quick, J., Loman, N. J., Duraffour, S., Simpson, J. T., Severi, E., Cowley, L., Bore, J. A., Koundouno, R., Dudas, G., Mikhail, A., Ouédraogo, N., Afrough, B., Bah, A., Baum, J. H. J., Becker-Ziaja, B., Boettcher, J. P., Cabeza-Cabrerizo, M., Camino-Sánchez, Á., Carter, L. L., Doerrbecker, J., Enkirch, T., Dorival, I. G., Hetzelt, N., Hinzmann, J., Holm, T., Kafetzopoulou, L. E., Koropogui, M., Kosgey, A., Kuisma, E., Logue, C. H., Mazzarelli, A., Meisel, S., Mertens, M., Michel, J., Ngabo, D., Nitzsche, K., Pallasch, E., Patrono, L. V., Portmann, J., Repits, J. G., Rickett, N. Y., Sachse, A., Singethan, K., Vitoriano, I., Yemanaberhan, R. L., Zekeng, E. G., Racine, T., Bello, A., Sall, A. A., Faye, O., Faye, O., Magassouba, N. F., Williams, C. V., Amburgey, V., Winona, L., Davis, E., Gerlach, J., Washington, F., Monteil, V., Jourdain, M., Bererd, M., Camara, A., Somlare, H., Camara, A., Gerard, M., Bado, G., Baillet, B., Delaune, D., Nebie, K. Y., Diarra, A., Savane, Y., Pallawo, R. B., Gutierrez, G. J., Milhano, N., Roger, I., Williams, C. J., Yattara, F., Lewandowski, K., Taylor, J., Rachwal, P., J. Turner, D., Pollakis, G., Hiscox, J. A., Matthews, D. A., Shea, M. K. O., Johnston, A. M., Wilson, D., Hutley, E., Smit, E., Di Caro, A., Wölfel, R., Stoecker, K., Fleischmann, E., Gabriel, M., Weller, S. A., Koivogui, L., Diallo, B., Keïta, S., Rambaut, A., Formenty, P., Günther, S., and Carroll, M. W. 2016. Real-time, portable genome sequencing for Ebola surveillance. Nature 530:228-232.

Raffaele, S., and Kamoun, S. 2012. Genome evolution in filamentous plant pathogens: why bigger can be better. Nature Reviews Microbiology 10:417-430.

Rovenich, H., Boshoven, J. C., and Thomma, B. P. H. J. 2014. Filamentous pathogen effector functions: of pathogens, hosts and microbiomes. Current Opinion in Plant Biology 20:96-103.

Saunders, D. G. O., Win, J., Cano, L. M., Szabo, L. J., Kamoun, S., and Raffaele, S. 2012. Using hierarchical clustering of secreted protein families to classify and rank candidate effectors of rust fungi. PLoS One 7:e29847. 
Schirawski, J., Mannhaupt, G., Munch, K., Brefort, T., Schipper, K., Doehlemann, G., Di Stasio, M., Rossel, N., Mendoza-Mendoza, A., Pester, D., Muller, O., Winterberg, B., Meyer, E., Ghareeb, H., Wollenberg, T., Munsterkotter, M., Wong, P., Walter, M., Stukenbrock, E., Guldener, U., and Kahmann, R. 2010. Pathogenicity determinants in smut fungi revealed by genome comparison. Science 330:1546-1548.

Schmidt, S. M., Lukasiewicz, J., Farrer, R., van Dam, P., Bertoldo, C., and Rep, M. C. 2016. Comparative genomics of Fusarium oxysporum f. sp. melonis reveals the secreted protein recognized by the Fom-2 resistance gene in melon. New Phytologist 209:307-318.

Schornack, S., van Damme, M., Bozkurt, T. O., Cano, L. M., Smoker, M., Thines, M., Gaulin, E., Kamoun, S., and Huitema, E. 2010. Ancient class of translocated oomycete effectors targets the host nucleus. Proceedings of the National Academy of Sciences of the United States of America 107:17421-17426.

Seidl, M. F., and Thomma, B. P. H. J. 2014. Sex or no sex: Evolutionary adaptation occurs regardless. BioEssays 36:335-345.

Seidl, M. F., Faino, L., Shi-Kunne, X., van den Berg, G. C., Bolton, M. D., and Thomma, B. P. H. J. 2015. The genome of the saprophytic fungus Verticillium tricorpus reveals a complex effector repertoire resembling that of its pathogenic relatives. Molecular Plant-Microbe Interactions 28:362-373.

Selker, E. U. 2002. Repeat-induced gene silencing in fungi. Advances in Genetics 46:439450.

Shin, S. C., Ahn, D. H., Kim, S. J., Lee, H., Oh, T.-J., Lee, J. E., and Park, H. 2013. Advantages of single-molecule real-time sequencing in high-GC content genomes. PLoS One 8:e68824.

Simpson, A. J., Reinach, F. C., Arruda, P., Abreu, F. A., Acencio, M., Alvarenga, R., Alves, L. M., Araya, J. E., Baia, G. S., Baptista, C. S., Barros, M. H., Bonaccorsi, E. D., Bordin, S., Bove, J. M., Briones, M. R., Bueno, M. R., Camargo, A. A., Camargo, L. E., Carraro, D. M., Carrer, H., Colauto, N. B., Colombo, C., Costa, F. F., Costa, M. C., Costa-Neto, C. M., Coutinho, L. L., Cristofani, M., Dias-Neto, E., Docena, C., El-Dorry, H., Facincani, A. P., Ferreira, A. J., Ferreira, V. C., Ferro, J. A., Fraga, J. S., Franca, S. C., Franco, M. C., Frohme, M., Furlan, L. R., Garnier, M., Goldman, G. H., Goldman, M. H., Gomes, S. L., Gruber, A., Ho, P. L., Hoheisel, J. D., Junqueira, M. L., Kemper, E. L., Kitajima, J. P., Krieger, J. E., Kuramae, E. E., Laigret, F., Lambais, M. R., Leite, L. C., Lemos, E. G., Lemos, M. V., Lopes, S. A., Lopes, C. R., Machado, J. A., Machado, M. A., Madeira, A. M., Madeira, H. M., Marino, C. L., Marques, M. V., Martins, E. A., Martins, E. M., Matsukuma, A. Y., Menck, C. F., Miracca, E. C., Miyaki, C. Y., Monteriro-Vitorello, C. B., Moon, D. H., Nagai, M. A., Nascimento, A. L., Netto, L. E., Nhani, A., Jr., Nobrega, F. G., Nunes, L. R., Oliveira, M. A., de Oliveira, M. C., de Oliveira, R. C., Palmieri, D. A., Paris, A., Peixoto, B. R., Pereira, G. A., Pereira, H. A., Jr., Pesquero, J. B., Quaggio, R. B., Roberto, P. G., Rodrigues, V., de, M. R. A. J., de Rosa, V. E., Jr., de Sa, R. G., Santelli, R. V., Sawasaki, H. E., da Silva, A. C., da Silva, A. M., da Silva, F. R., da Silva, W. A., Jr., da Silveira, J. F., Silvestri, M. L., Siqueira, W. J., de Souza, A. A., de Souza, A. P., Terenzi, M. F., Truffi, D., Tsai, S. M., Tsuhako, M. H., Vallada, H., Van Sluys, 
M. A., Verjovski-Almeida, S., Vettore, A. L., Zago, M. A., Zatz, M., Meidanis, J., and Setubal, J. C. 2000. The genome sequence of the plant pathogen Xylella fastidiosa. The Xylella fastidiosa consortium of the organization for nucleotide sequencing and analysis. Nature 406:151-159.

Sperschneider, J., Dodds, P. N., Gardiner, D. M., Manners, J. M., Singh, K. B., and Taylor, J. M. 2015. Advances and challenges in computational prediction of effectors from plant pathogenic fungi. PLoS Pathogens 11:e1004806.

Sperschneider, J., Gardiner, D. M., Dodds, P. N., Tini, F., Covarelli, L., Singh, K. B., Manners, J. M., and Taylor, J. M. 2016. EffectorP: predicting fungal effector proteins from secretomes using machine learning. New Phytologist 210:743-761.

Stergiopoulos, I., and de Wit, P. 2009. Fungal effector proteins. Annual Review of Phytopathology 47:233-263.

Stergiopoulos, I., Collemare, J., Mehrabi, R., and De Wit, P. J. G. M. 2013. Phytotoxic secondary metabolites and peptides produced by plant pathogenic Dothideomycete fungi. FEMS Microbiology Reviews 37:67-93.

Stukenbrock, E. H., and McDonald, B. A. 2008. The origins of plant pathogens in agroecosystems. Annual Review of Phytopathology 46:75-100.

Thomma, B. P. H. J., Seidl, M. F., Shi-Kunne, X., Cook, D. E., Bolton, M. D., van Kan, J. A. L., and Faino, L. 2016. Mind the gap; seven reasons to close fragmented genome assemblies. Fungal Genetics and Biology 90:24-30.

Torto, T. A., Li, S., Styer, A., Huitema, E., Testa, A., Gow, N. A., van West, P., and Kamoun, S. 2003. EST mining and functional expression assays identify extracellular effector proteins from the plant pathogen Phytophthora. Genome Research 13:1675-1685.

Tyler, B. M., Tripathy, S., Zhang, X., Dehal, P., Jiang, R. H., Aerts, A., Arredondo, F. D., Baxter, L., Bensasson, D., Beynon, J. L., Chapman, J., Damasceno, C. M., Dorrance, A. E., Dou, D., Dickerman, A. W., Dubchak, I. L., Garbelotto, M., Gijzen, M., Gordon, S. G., Govers, F., Grunwald, N. J., Huang, W., Ivors, K. L., Jones, R. W., Kamoun, S., Krampis, K., Lamour, K. H., Lee, M. K., McDonald, W. H., Medina, M., Meijer, H. J., Nordberg, E. K., Maclean, D. J., Ospina-Giraldo, M. D., Morris, P. F., Phuntumart, V., Putnam, N. H., Rash, S., Rose, J. K., Sakihama, Y., Salamov, A. A., Savidor, A., Scheuring, C. F., Smith, B. M., Sobral, B. W., Terry, A., Torto-Alalibo, T. A., Win, J., Xu, Z., Zhang, H., Grigoriev, I. V., Rokhsar, D. S., and Boore, J. L. 2006. Phytophthora genome sequences uncover evolutionary origins and mechanisms of pathogenesis. Science 313:1261-1266.

van Schie, C. C., and Takken, F. L. 2014. Susceptibility genes 101: how to be a good host. Annual Review of Phytopathology 52:551-581.

Vleeshouwers, V. G. A. A., Rietman, H., Krenek, P., Champouret, N., Young, C., Oh, S.K., Wang, M., Bouwmeester, K., Vosman, B., Visser, R. G. F., Jacobsen, E., Govers, F., Kamoun, S., and Van der Vossen, E. A. G. 2008. Effector genomics accelerates discovery and functional profiling of potato disease resistance and Phytophthora Infestans avirulence genes. PLoS One 3:e2875.

von Heijne, G. 1990. The signal peptide. The Journal of Membrane Biology 115:195-201. 
Whisson, S. C., Boevink, P. C., Moleleki, L., Avrova, A. O., Morales, J. G., Gilroy, E. M., Armstrong, M. R., Grouffaud, S., van West, P., Chapman, S., Hein, I., Toth, I. K., Pritchard, L., and Birch, P. R. J. 2007. A translocation signal for delivery of oomycete effector proteins into host plant cells. Nature 450:115-118.

Williams, A. H., Sharma, M., Thatcher, L. F., Azam, S., Hane, J. K., Sperschneider, J., Kidd, B. N., Anderson, J. P., Ghosh, R., Garg, G., Lichtenzveig, J., Kistler, H. C., Shea, T., Young, S., Buck, S.-A. G., Kamphuis, L. G., Saxena, R., Pande, S., Ma, L.-J., Varshney, R. K., and Singh, K. B. 2016. Comparative genomics and prediction of conditionally dispensable sequences in legume-infecting Fusarium oxysporum formae speciales facilitates identification of candidate effectors. BMC genomics 17:1-24.

Yandell, M., and Ence, D. 2012. A beginner's guide to eukaryotic genome annotation. Nature Reviews Genetics 13:329-342. 



\section{Chapter 3}

$$
\text { do }
$$

$$
26
$$

ᄂ 


\section{Verticillium dahliae strains that infect the same host plant display highly divergent effector catalogs}

Hesham A.Y. Gibriel ${ }^{1,2, \$}$, Jinling Li ${ }^{1, \$}$, Longfu Zhu ${ }^{3}$, Michael F. Seidl11,\#, Bart P.H.J. Thomma ${ }^{1, \#}$

1 Laboratory of Phytopathology, Wageningen University \& Research, 6708 PB Wageningen, The Netherlands.

2 Current address: School of Agriculture and Food Science, University College Dublin, Dublin, Ireland.

3 National Key Laboratory of Crop Genetic Improvement, Huazhong Agricultural University, Wuhan, Hubei 430070, China.

\$ These authors contributed equally

\# These authors also contributed equally to this work

To be submitted for publication 


\begin{abstract}
Effectors are proteins secreted by pathogens to support colonization of host plants, often by deregulating host immunity. Effector genes are often localized within dynamic lineage-specific (LS) genomic regions, allowing rapid evolution of effector catalogues. It is thought that such localization permits pathogens to be competitive in the coevolutionary arms races with their hosts. For a broad host-range pathogen such as Verticillium dahliae it is unclear to what extent single members of their total effector repertoires contribute to disease development on multiple hosts. Here, we determined the core and LS effector repertoires of a collection of $V$. dahliae strains, as well as the ability of these strains to infect a range of plant species comprising tomato, cotton, Nicotiana benthamiana, Arabidopsis, and sunflower to assess whether the presence of particular LS effectors correlates with the ability to infect particular plant species. Surprisingly, we found that $V$. dahliae strains that are able to infect the same host plant harbor highly divergent LS effector repertoires. Furthermore, we observed differential $V$. dahliae core effector gene expression between host plants. Our data suggest that different $V$. dahliae lineages utilise divergent effector catalogs to colonize the same host plant, suggesting considerable redundancy among the activities of effector catalogs between lineages.
\end{abstract}




\section{INTRODUCTION}

Plant pathogens cause devastating diseases on crop plants, threatening food security worldwide (Fisher et al. 2012; Pennisi 2010). In order to establish their infection, pathogens secrete effector molecules that can modulate host physiology, often by deregulating host immune responses (Cook et al. 2015; Jones and Dangl 2006). However, in turn, effectors may become recognized by plant immune receptors, leading to the activation of immune responses and attempted arrest of pathogen invasion (Cook et al. 2015; Jones and Dangl 2006). Thus, pathogens need to continuously evolve their effector catalogues by modifying or purging existing effectors that became recognized, or by acquiring novel effectors to suppress effector-triggered immune responses (Cook et al. 2015; Jones and Dangl 2006).

Genomes of plant pathogens are often thought to have evolved two distinct compartments; one comprising gene-rich, repeat-poor genomic regions that contain core genes that mediate general physiology, and one comprising gene-poor, plastic, repeat-rich genomic regions that contain effector genes and other pathogenicity-related genes (Dong et al. 2015; Raffaele and Kamoun 2012). The plastic genomic regions are either embedded within core chromosomes or reside on separate chromosomes that are often referred to as conditionally dispensable chromosomes (CDCs) (Dong et al. 2015; Raffaele and Kamoun 2012). For instance, effector genes of the tomato-pathogen Fusarium oxysporum f. sp. lycopersici, known as secreted in xylem (SIX) genes, are located on dispensable chromosomes (Schmidt et al. 2013). In contrast, all known effectors of the fungal wheat pathogen Zymoseptoria tritici are located on core chromosomes, while no recognizable effector genes reside on dispensable chromosomes (Kema et al. 2018; Marshall et al. 2011; Meile et al. 2018). Core chromosomes also carry effector genes in other fungal plant pathogens, such as the fungal smut pathogen Ustilago maydis and the fungal tomato pathogen Cladosporium fulvum (Hemetsberger et al. 2015; Stergiopoulos et al. 2010). A genome compartmentalization with physically separated effector-containing regions is often referred to as a "two-speed" genome organization because it is thought that gene-rich, repeat-poor genomic regions evolve slowly, while gene-poor, repeat-rich genomic regions evolve quicker (Croll and McDonald 2012; Raffaele and Kamoun 2012). Accordingly, the occurrence of effector genes within plastic genomic regions allows rapid evolution of effector catalogues and permits pathogens to be competitive in the co-evolution with hosts and evade their immune systems (Dong et al. 2015; Raffaele and Kamoun 2012).

Verticillium dahliae is a soil-borne fungal plant pathogen that is able to infect a broad range of plant species, including crops such as tomato, potato, lettuce, and cotton (Fradin and Thomma 2006; Inderbitzin et al. 2011). The fungus infects plants through their roots and subsequently colonizes the water-conducting xylem vessels, leading to vascular wilt disease (Fradin and Thomma 2006). Comparative genomics between closely related $V$. dahliae strains revealed that they carry highly dynamic, repeat rich, lineage-specific (LS) 
regions that are only present in a subset of $V$. dahliae strains, and that account for up to $4 \mathrm{Mb}$ of the $35 \mathrm{Mb}$ genome (de Jonge et al. 2013; Faino et al. 2016). These LS regions are enriched for in planta-induced effector genes that contribute to fungal virulence (de Jonge et al. 2013). However, effector genes are not only found in LS regions, as also the core genome harbors effector genes, such as those encoding a family of necrosis and ethylene-inducing-like proteins (NLPS) some of which were found to induce cell death in dicotyledonous plants (de Jonge et al. 2011; Santhanam et al. 2013). Similarly, also a family of lysin motif (LysM) effectors is encoded in the core genome, various homologs of which have been reported to enhance virulence by suppression of chitintriggered immunity in other fungal pathogens (de Jonge et al. 2010; Kombrink et al. 2017; Marshall et al. 2011; Mentlak et al. 2012; Takahara et al. 2016). However, only a single LysM effector that is encoded in an LS region of $V$. dahliae strain VdLs17, and is thus not identified in the genomes of other $V$. dahliae strains, was found to contribute to virulence by suppression of chitin-triggered immunity, whereas no role in virulence could be attributed to any of the core LysM effectors (Kombrink et al. 2017).

Whereas $V$. dahliae is characterized by its generally broad host range, differential pathogenicity among hosts occurs for individual strains (Bhat and Subbarao 1999). In this study, we analysed the genomes of a collection of $V$. dahliae strains and assessed their core and LS effector catalogues in relation to their host ranges. To this end, we selected a set of strains that are well-adapted to cause disease on tomato (Solanum lycopersicum), cotton (Gossypium hirsutum), Australian tobacco (Nicotiana benthamiana), Arabidopsis (Arabidopsis thaliana), and sunflower (Helianthus annuus) and determined their core and LS effector catalogues as well as their in planta expression profiles.

\section{MATERIALS AND METHODS}

\section{V. dahliae strains and plant inoculations}

In total, $21 \mathrm{~V}$. dahliae strains collected at different geographical locations were used in this study (Table S1). All strains were grown on potato dextrose agar (PDA; Oxoid, Basingstoke, UK) at $22^{\circ} \mathrm{C}$, and conidiospores were collected from 10-day-old plates and washed with tap water. Disease assays were performed on sunflower (Helianthus annuus L. cv. Tutti), cotton (Gossypium hirsutum cv. Simian 3), tomato (Solanum lycopersicum cv. Moneymaker), Nicotiana benthamiana and Arabidopsis thaliana (Col-0) plants using the root-dipping inoculation method as previously described (Fradin et al. 2009; Song et al. 2017). Briefly, two-week-old (Arabidopsis, tomato, cotton, sunflower) or threeweek-old ( $N$. benthamiana) seedlings were carefully uprooted and the roots were rinsed in water. Subsequently, the roots were dipped for eight minutes in a suspension of $10^{6}$ conidiospores $/ \mathrm{mL}$ of water. Control plants were treated similarly by root dipping in tap water without conidiospores. Disease symptoms were scored up to 21 (tomato, N. benthamiana, Arabidopsis), 28 (cotton) or 45 (sunflower) days post inoculation (dpi). 


\section{Genome sequencing and assembly}

The genome sequences of $13 \mathrm{~V}$. dahliae strains were previously determined, nine of which were previously sequenced using Illumina HiSeq 2000 (de Jonge et al. 2013; de Jonge et al. 2012) and four that were sequenced using long-read PacBio SingleMolecule Real-Time (SMRT) sequencing technology (Table S1) (Faino et al. 2015). Additionally, eight $V$. dahliae strains (Table S1) were newly sequenced in this study. To this end, genomic DNA of $V$. dahliae strains was obtained from conidiospores that were harvested from ten-day-old cultures grown on potato dextrose agar as described previously (de Jonge et al. 2012). Library preparation ( 500 bp insert size) and genomic sequencing (100 bp paired-end reads) were performed at the Beijing Genome Institute (BGl, Hong Kong). All V. dahliae strains that were sequenced with short-read sequencing technology (17 strains) were assembled with the A5 pipeline (default parameters) that automates data cleaning, error correction, assembly, and quality control (Tritt et al. 2012). Genome assembly statistics for all $21 \mathrm{~V}$. dahliae strains were calculated using QUAST (Gurevich et al. 2013). Repeats were identified using RepeatModeler (version 1.0.8) (default parameters) (Smit and Hubley 2010) and masked using RepeatMasker (version 4.0.7) (default parameters) (Smit et al. 2016).

\section{Gene prediction and annotation}

Previously generated gene annotations of $V$. dahliae strains JR2 (Faino et al. 2015) and CQ2 (unpublished data) were used in this study. For the remaining $19 \mathrm{~V}$. dahliae strains, gene annotation was performed using the Maker2 pipeline (Holt and Yandell 2011) that combines ab initio protein-coding gene evidence from SNAP (Korf 2004), Augustus (Stanke and Waack 2003), and GeneMark-HMM (Lukashin and Borodovsky 1998). Additionally, Maker2 was provided with the previously generated reference gene annotation of $V$. dahliae strain JR2 (Faino et al. 2015), gene annotation of $V$. dahliae strain CQ2 (unpublished data), and protein homologs of 260 predicted fungal proteomes obtained from the UniProt database (Apweiler et al. 2004).

\section{Effector profiling}

We determined core and LS regions of each $V$. dahliae strain. For LS regions, pairwise whole-genome alignments of the $21 \mathrm{~V}$. dahliae strains were performed using NUCmer (version 3.1) (--maxmatch), which is part of the MUMer package (Kurtz et al. 2004), and LS regions (here defined as genomic regions that are shared by $<19 \mathrm{~V}$. dahliae strains) were extracted. Subsequently, core regions (regions shared by $\geq 19 \mathrm{~V}$. dahliae strains) were determined. Genes localized within core and LS regions were extracted using BEDtools intersect (Quinlan and Hall 2010).

To identify candidate effectors, $\mathrm{N}$-terminal signal peptides were first predicted with SignalP (version 4.1) (Petersen et al. 2011). Subsequently, the machine-learning approach applied in EffectorP (version 1.0) (default parameters) was used (Sperschneider et al. 2016). Effector genes localized within core and LS regions were extracted using BEDtools intersect (Quinlan and Hall 2010). Sequence similarity between predicted LS effectors 
was established by an all-vs.-all analyses using BLASTp (E-value cutoff 1e-5) (Altschul et al. 1990). Clustering of LS effector sequences into different families was performed using MCL (default options) (Li et al. 2003) and visualized using the R package pheatmap (Kolde 2015).

\section{Assessment of gene expression}

To assess gene expression levels, two RNA-seq datasets were used. The first RNAseq dataset was previously generated from $V$. dahliae strain JR2-infecting Nicotiana benthamiana plants at 4, 8, 12, and 16 dpi (de Jonge et al. 2012; Faino et al. 2014). The second RNA-seq dataset was obtained from $V$. dahliae strain V991 infecting Gossypium hirsutum (cotton) plants at 6, 9, 12, and 15 dpi (L. Zhu, unpublished data). Mapping of RNA-seq datasets to the corresponding genomes was performed using STAR (version 2.5.3) (--runThreadN 16) (Dobin et al. 2013), and gene expression levels were determined using RSEM (version 1.2.3) (calculate-expression command) (default parameters) (Li and Dewey 2011), by calculating transcripts per million (TPM) for each gene in each sample.

\section{RESULTS}

\section{Verticillium dahliae pathogenicity on a panel of potential host plants}

To evaluate the pathogenicity of a collection of $V$. dahliae strains on a panel of potential host plant species, we conducted inoculation experiments with 21 strains on the Solanaceae crop plant tomato and model plant $N$. benthamiana, the Malvaceae crop plant cotton, the Asteraceae crop plant sunflower, and the Brassicaceae model plant Arabidopsis. Despite the fact that $V$. dahliae is often considered a broad host range pathogen, there is no individual $V$. dahliae strain in this collection that is able to cause disease on all tested plant species (Table 1). All isolates are pathogenic on Arabidopsis (Figure 1) and on $N$. benthamiana (Figure 2), albeit that the severity of disease symptoms induced by different strains vary considerably (Figure 1 \& Figure 2). Most strains were also found to cause disease on cotton, with the exception of strains 2009-605 and V152 that are non-pathogenic on this host species (Figure 3). Interestingly, $V$. dahliae strains CQ2, 463 and ST100 cause severe defoliation, while the other pathogenic strains induce mild to moderate disease symptoms that include wilting, stunting and chlorosis in the absence of defoliation (Figure 3). Fewer $V$. dahliae strains are able to cause disease on tomato (Figure 4). Besides several strains that cause defoliation on cotton, the tomato non-pathogenic strains also include several non-defoliators on cotton like Vd39 and $85 \mathrm{~S}$ (Figure 3 \& Figure 4). Interestingly, whereas strain V152 that is non-pathogenic on cotton also fails to cause disease on tomato, strain 2009-605 that is non-pathogenic on cotton is able to cause wilt disease on tomato (Figure 4). Strikingly, except for $V$. dahliae strain $85 \mathrm{~S}$ that induces clear wilt disease symptoms on sunflower plants, including stunting, chlorosis and necrosis, all other strains fail to cause disease on sunflower (Figure 5). 
Thus, differential pathogenicity occurs within the collection of $V$. dahliae strains tested here.

Table 1. Inoculation experiments with a collection of $V$. dahliae strains on a collection of potential host plants.

\begin{tabular}{|c|c|c|c|c|c|}
\hline Strain & Arabidopsis & N. benthamiana & Cotton & Tomato & Sunflower \\
\hline ST100 & + & + & + & - & - \\
\hline 463 & + & + & + & - & - \\
\hline $\mathrm{CQ} 2$ & + & + & + & - & - \\
\hline Vd39 & + & + & + & - & - \\
\hline $85 S$ & + & + & + & - & + \\
\hline JKG8 & + & + & + & + & - \\
\hline DVD-S94 & + & + & + & + & - \\
\hline DVD3 & + & + & + & + & - \\
\hline DVD31 & + & + & + & + & - \\
\hline DVD161 & + & + & + & + & - \\
\hline DVD-S29 & + & + & + & + & - \\
\hline ST14.01 & + & + & + & + & - \\
\hline CBS38166 & + & + & + & + & - \\
\hline DVD-S26 & + & + & + & + & - \\
\hline Vandijk & + & + & + & - & - \\
\hline ST16.01 & + & + & + & - & - \\
\hline 2009-605 & + & + & - & + & - \\
\hline V152 & + & + & - & - & - \\
\hline V52 & + & + & + & + & - \\
\hline VdLs17 & + & + & + & + & - \\
\hline JR2 & + & + & + & + & - \\
\hline
\end{tabular}

' + '= pathogenic; '-'= non-pathogenic. Disease symptoms were scored up to 21 days post inoculation (dpi) (tomato, N. benthamiana, Arabidopsis), $28 \mathrm{dpi}$ (cotton) or $45 \mathrm{dpi}$ (sunflower). The inoculation experiments were executed twice with similar results. 


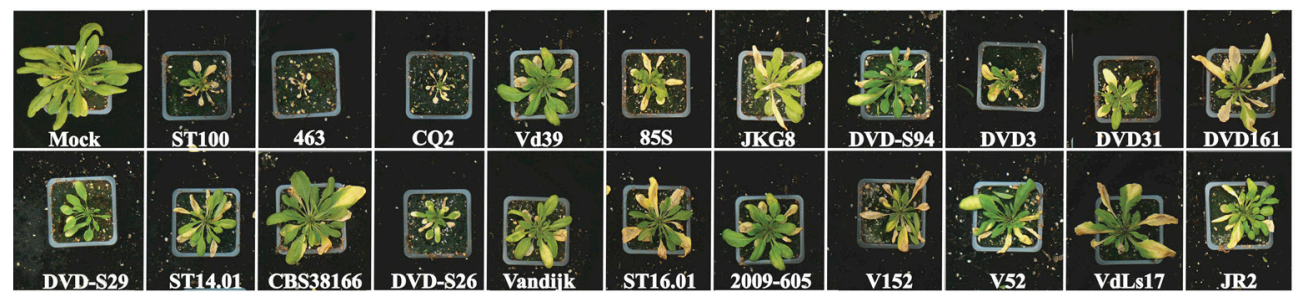

Figure 1. Phenotypes of Arabidopsis thaliana inoculated with $\mathbf{V}$. dahliae strains. Typical appearance of $A$. thaliana (Col-0) plants upon mock-inoculation or inoculation with a collection of $V$. dahliae strains. All inoculated $V$. dahliae strains are pathogenic on $A$. thaliana. Note that disease symptoms range from stunting, wilting to severe tissue necrosis. Pictures show representative plants at 21 days after inoculation taken from one of two independent inoculation experiments.

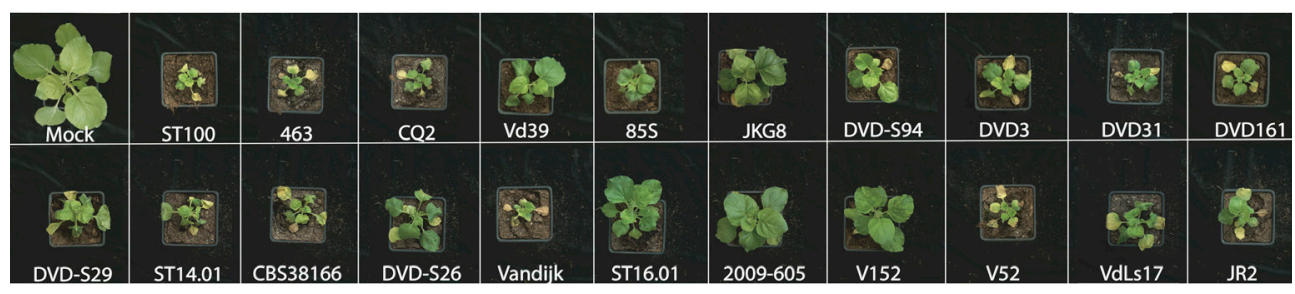

Figure 2. Phenotypes of Nicotiana benthamiana inoculated with $\boldsymbol{V}$. dahliae strains. Typical appearance of $N$. benthamiana plants upon mock-inoculation or inoculation with a collection of $V$. dahliae strains. All inoculated $V$. dahliae strains are pathogenic on $N$. benthamiana. Note that disease symptoms include stunting, wilting and severe tissue necrosis. Pictures show representative plants at 21 days after inoculation taken from one of two independent inoculation experiments.

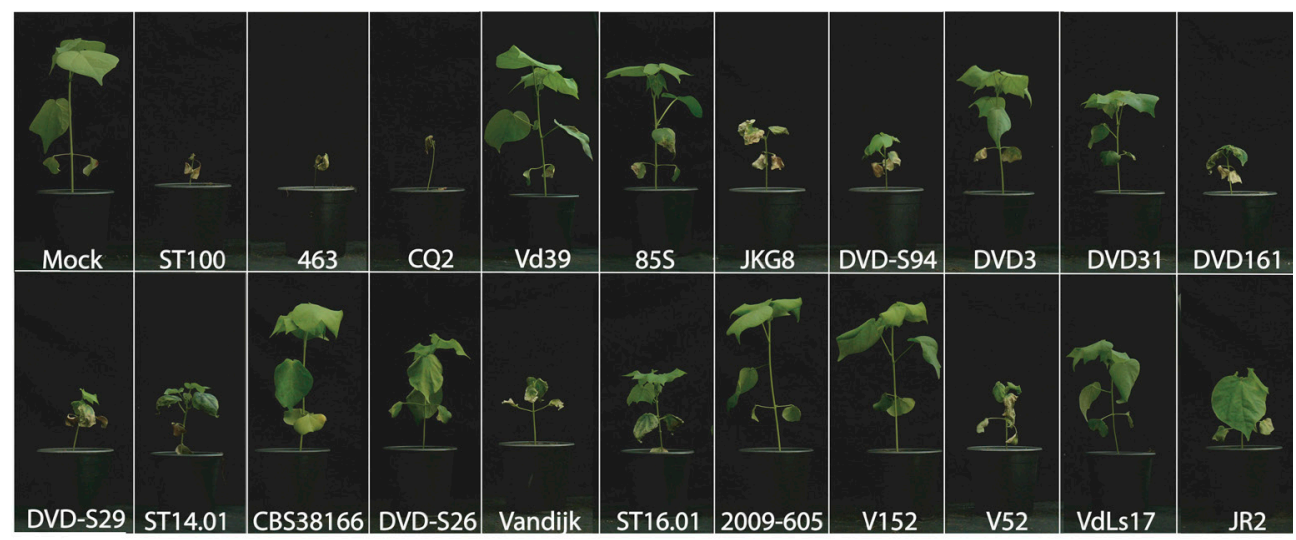

Figure 3. Phenotypes of cotton plants inoculated with $\mathbf{V}$. dahliae strains. Typical appearance of cotton (cv. Simian 3) plants upon mock-inoculation or inoculation with a collection of $V$. 
dahliae strains. $V$. dahliae strains display differential pathogenticity on cotton plants. Note that several $V$. dahliae strains cause defoliation symptoms, while others induce wilting, stunting but not defoliation. Pictures show representative plants at 28 days after inoculation taken from one of two independent inoculation experiments.

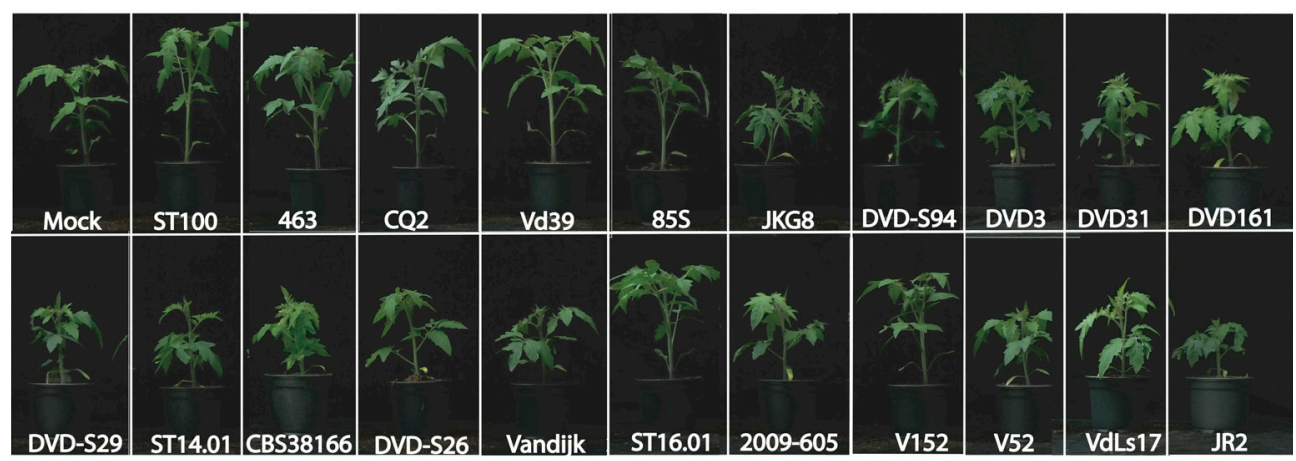

Figure 4. Phenotypes of tomato plants inoculated with $\boldsymbol{V}$. dahliae strains. Typical appearance of tomato (cv. Moneymaker) plants upon mock-inoculation or inoculation with a collection of $V$. dahliae strains. $V$. dahliae strains display differential pathogenticity on tomato plants. Note that pathogenic strains induce clear stunting and significant reduction in canopy area development on inoculated plants. Pictures show representative plants at 21 days after inoculation taken from one of two independent inoculation experiments. 


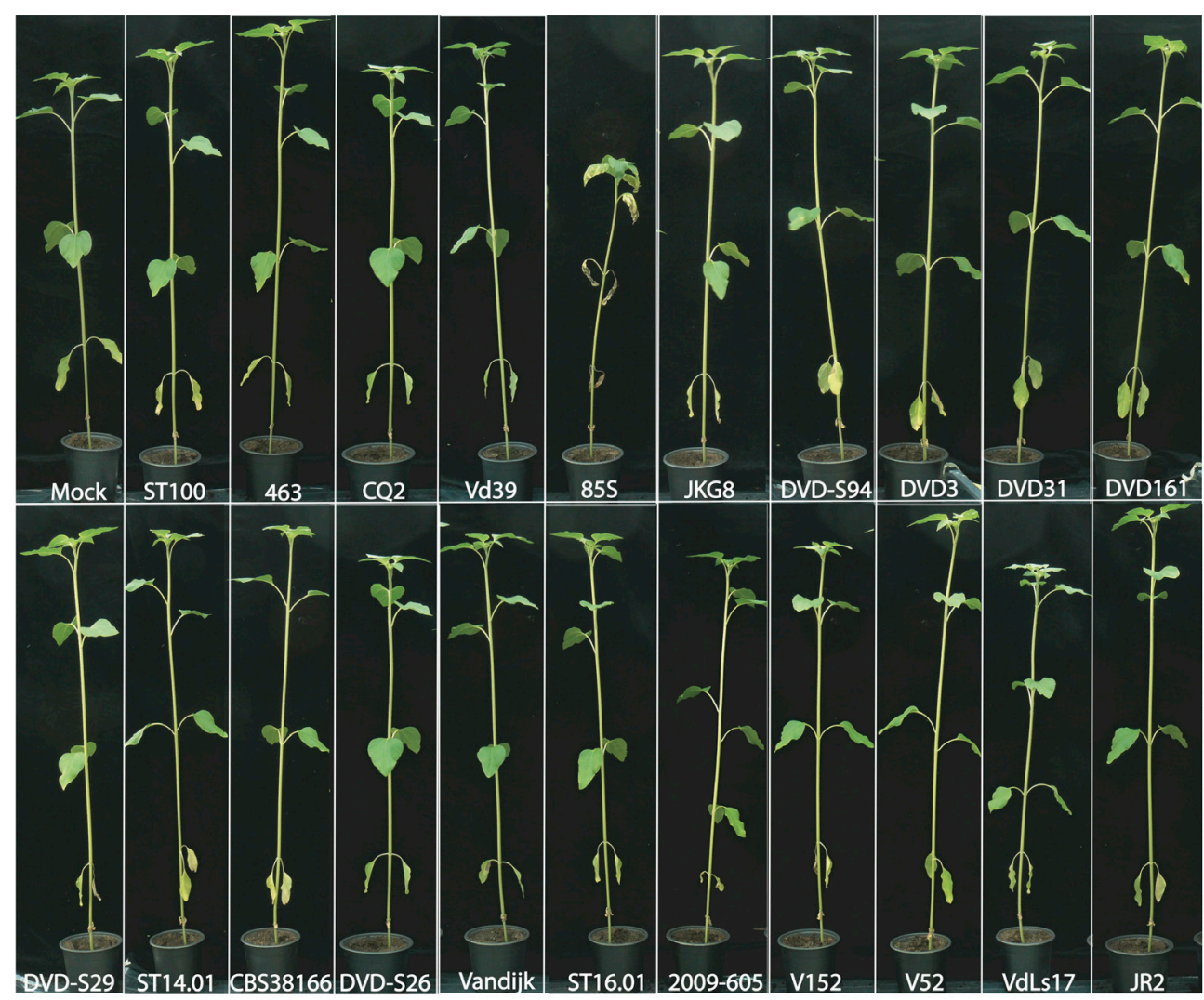

Figure 5. Phenotypes of sunflower plants inoculated with $\mathbf{V}$. dahliae strains. Typical appearance of sunflower (cv. Tutti) plants upon mock-inoculation or inoculation with a collection of $V$. dahliae strains. $V$. dahliae strains display differential pathogenticity on sunflower plants. Note that besides the stunting, the plant inoculated with pathogenic strain $85 \mathrm{~S}$ also displays chlorosis and wilting symptoms. Pictures show representative plants at 45 days after inoculation taken from one of two independent inoculation experiments.

\section{Genome assemblies and annotations of a collection of $\boldsymbol{V}$. dahliae strains}

The genome sequences of $13 \mathrm{~V}$. dahliae strains (Table S1) were obtained from previous studies, nine of which were determined using the Illumina HiSeq 2000 platform (de Jonge et al. 2013; de Jonge et al. 2012), and four were sequenced using long-read PacBio Single-Molecule Real-Time (SMRT) sequencing technology (Faino et al. 2015). In this study, we sequenced the genomes of eight additional $V$. dahliae strains (Table S1) using the Illumina HiSeq 2000 platform, yielding 1.1 Gb of paired-end (PE) libraryderived reads (500 bp insert size; $100 \mathrm{bp}$ read length) per strain. As the genomes of $V$. dahliae strains that were previously sequenced with Illumina technology showed a reduced N50 size of about $35.55 \mathrm{~kb}$ (de Jonge et al. 2012), all the Illumina sequenced genomes were re-assembled in this study. The short reads of the $17 \mathrm{~V}$. dahliae strains 
that were sequenced with Illumina technology were assembled into $~ 34 \mathrm{Mb}$, with the largest assembly of $35.90 \mathrm{Mb}$ for $\mathrm{V}$. dahliae strain $\mathrm{Vd} 39$, and the smallest assembly of 33.14 Mb for $V$. dahliae strain DVD-S29 (Table S2). All assemblies comprised between 1,000 and 4,188 scaffolds with an N50 of $\sim 50 \mathrm{~kb}$, except for $V$. dahliae strains 463 and V52 that were assembled in 4,188 and 3,419 scaffolds with an N50 of $17.74 \mathrm{~kb}$ and $21.80 \mathrm{~kb}$, respectively (Table S2).

To assess the completeness of the assemblies, the Benchmarking Universal Single-Copy Orthologs (BUSCO) software was used, which uses a set of 1,315 core Ascomycota genes as queries (Simão et al. 2015). The BUSCO scores amounted to 91\% for all the assemblies, except for $V$. dahliae strains 463 and v52 that resulted in $75 \%$ and $78.6 \%$, respectively (Table S2). For the PacBio sequenced strains, BUSCO scored $99.30 \%$ for $85 \mathrm{~S}$ and $97.50 \%$ for CQ2, while the BUSCO scores for the gapless genome assemblies of JR2 and VdLs17 (Faino et al. 2015) amounted to $99.40 \%$ and $98.90 \%$, respectively (Table S2).

Repetitive elements are strong drivers of genome evolution in plant pathogens (Seidl and Thomma 2017). Thus, the amounts and types of repetitive elements in the genomes of $V$. dahliae strains were predicted by combining de novo and known repetitive elements with RepeatMasker (Smit et al. 2016). The repeat content within the $V$. dahliae genomes varied between $6.64 \%$ (2.26 Mb) for $V$. dahliae strain $2009-605$ and $13.43 \%$ (4.83 $\mathrm{Mb}$ ) for $V$. dahliae strain $85 \mathrm{~S}$ (Table S3). Out of all the annotated repetitive elements, different repeat families were identified, which included long terminal repeats (LTRs) (2 Mb, 5.7\%), long interspersed nuclear elements (LINEs) (40 kb, $0.11 \%$ ), and short interspersed nuclear elements (SINEs) (2.9 kb, 0.01\%) (Table S3).

We subsequently inferred gene annotations for the various $V$. dahliae strains. For $V$. dahliae strains JR2 and CQ2, a previously determined gene annotation was used (Faino et al. 2015). The completeness of gene annotation for both strains was assessed using BUSCO that scored only a low score for CQ2 (72\%) compared to JR2 (90.80\%). Thus, gene annotations were inferred for all $20 \mathrm{~V}$. dahliae strains, except for $\mathrm{V}$. dahliae strain JR2. The Maker2 pipeline (Holt and Yandell 2011) was used that combines de novo, homology-based, and previous gene annotations for $V$. dahliae strains CQ2 (B. Thomma and J. Li, unpublished data) and JR2 (Faino et al. 2015), and protein homologs of 260 predicted fungal proteomes. The number of genes varied from 10,461 for $V$. dahliae strain VanDijk to 11,341 for $V$. dahliae v52 (Table 2).

\section{Identification of core and lineage-specific (LS) effector catalogs}

Initially, the secretomes for each of the $V$. dahliae strains were predicted, identifying between 1,002 secreted proteins for $V$. dahliae strain 463 and 1,108 proteins for $V$. dahliae strain ST16 (Table 2). Subsequently, the machine-learning algorithm of EffectorP (Sperschneider et al. 2016) was used, which identified between 169 effectors for $V$. dahliae strain CQ2 and 212 effectors for V. dahliae strain JR2 (Table 2). 


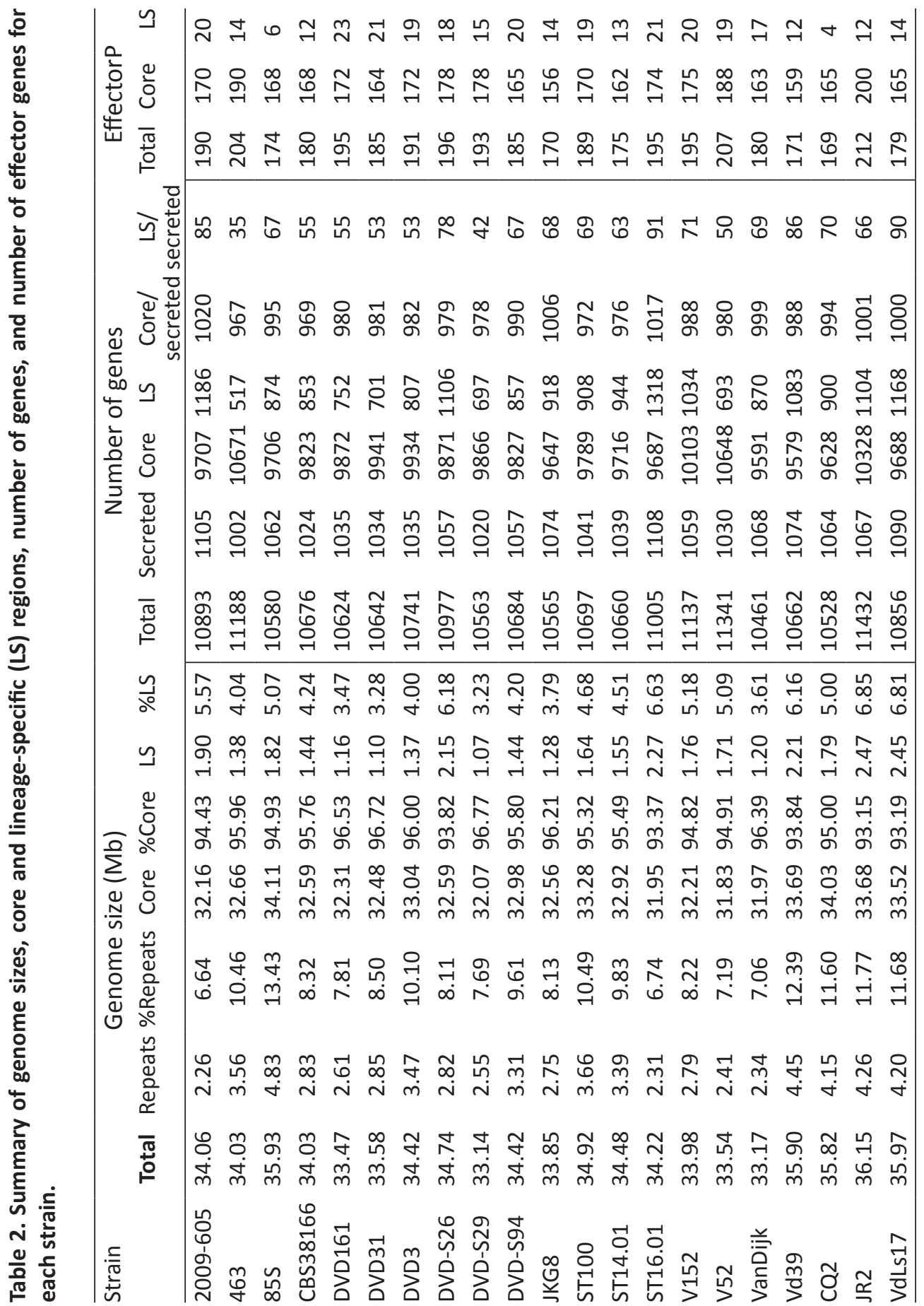


Subsequently, we determined the core genome, here defined as regions that are shared by $\geq 19 V$. dahliae strains, and LS regions, here defined as regions that are shared by $<19 \mathrm{~V}$. dahliae strains, for all $\mathrm{V}$. dahliae strains. The core regions of all $\mathrm{V}$. dahliae strains comprise $32.79 \mathrm{Mb}$ (93-97\%) of the genome, while LS regions comprise between 1.06 and $2.47 \mathrm{Mb}(3-7 \%)$ (Table 2). On average, the core regions of $V$. dahliae strains harbor 9,886 genes, comprising 988 genes that encode secreted proteins, of which 171 were classified as effectors based on EffectorP (Table 2). The LS regions of $V$. dahliae strains harbor between 517 genes for $V$. dahliae strain 463 and 1,318 genes for $V$. dahliae strain ST16 (Table 2). Of these LS genes, 35 genes encode secreted proteins for $V$. dahliae strain 463 and 91 for $V$. dahliae strain ST16, of which 15 genes were classified as effectors for each $V$. dahliae strain (Table 2). We tested these predictions on the previously identified LS effector gene of $V$. dahliae strain JR2, namely Ave1, which was successfully identified as a LS effector gene (de Jonge et al. 2012) (Figure S1).

To assess general characteristics of core and LS effector genes, features such as their distance to transposable elements (TEs), gene length, inter-genic length, and expression were determined. For all strains, we observed that LS effector genes are shorter in length than core effector genes (Figure 6A, B; Figure S2). The average inter-genic length of LS effector genes is slightly longer (1476 bp) compared to the average inter-genic length of core effector genes (1232 bp) (Figure 6A, B; Figure S3). Moreover, LS effector genes localize closer to TEs than core effector genes, even though this trend is not significant for $V$. dahliae strain JR2 (Figure 6A, B; Figure S4). Finally, LS effector genes of $V$. dahliae strain JR2 were found to be significantly higher expressed in planta on $\mathrm{N}$. benthamiana than core effector genes (Figure 7A), although no such difference was found between LS and core effector genes of $V$. dahliae strain CQ2 in planta on cotton (Figure 7B).

V. dahliae strains that infect the same host plant harbor divergent effector repertoires It has previously been shown that $V$. dahliae strains display differential capacity to infect particular host plants (Bhat and Subbarao 1999). We furthermore showed that especially LS effectors contribute to $V$. dahliae pathogenicity on individual plant hosts (de Jonge et al. 2013; de Jonge et al. 2012; Kombrink et al. 2017). Collectively, this suggests that $V$. dahliae strains may harbor an array of specialized effectors that only function on particular host plants. Therefore, we assessed whether the presence of particular LS effectors in the various $V$. dahliae strains correlates with the ability to infect particular hosts. In total, we predicted 333 LS effectors over the various strains (Table 2) that were clustered into 110 families that are either shared by sub-groups of $V$. dahliae strains or are strain-specific (Figure 8). Of these effectors, the previously identified LS effector gene Ave 1 is shared by sub-groups of $V$. dahliae strains (de Jonge et al. 2013), while the Sun 1 effector gene of $V$. dahliae strain $85 S$ is strain specific (Chapter 4) (Figure 8). Notably, in contrast to previous observations, we found that the LS effector genes XLOC_00170, XLOC_008951, and XLOC_009059 were only present in V. dahliae strain JR2 and not in additional $V$. dahliae strains (de Jonge et al. 2013). 


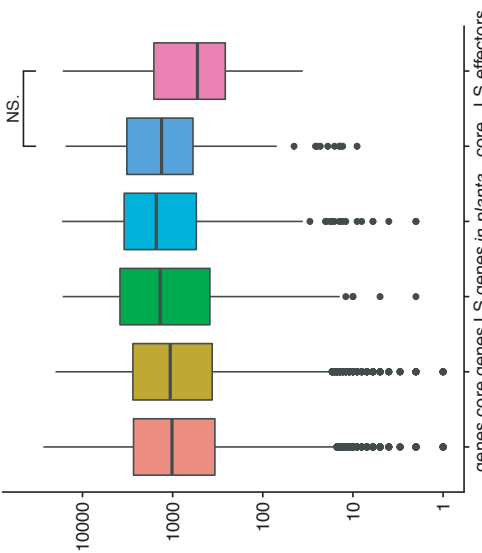

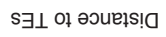

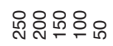

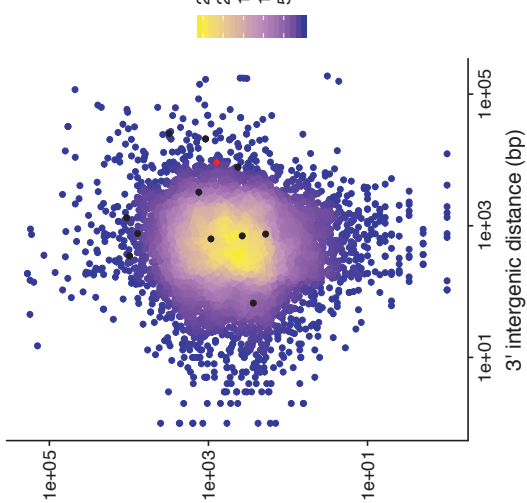

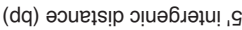

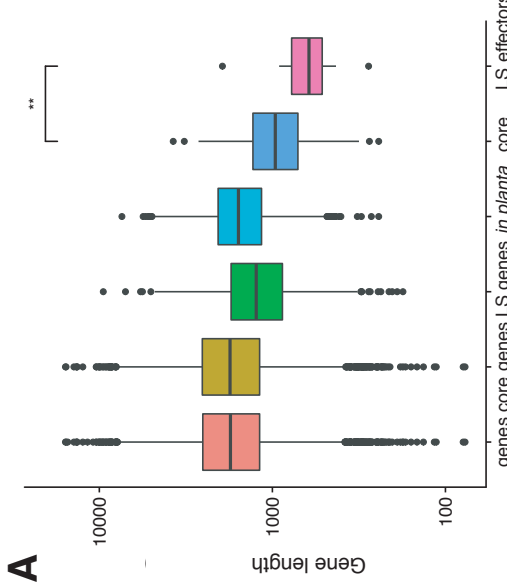

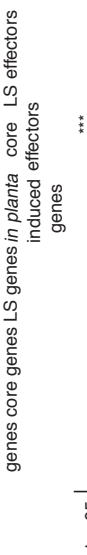

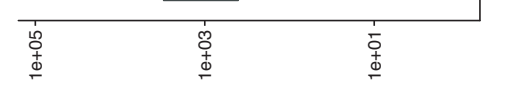

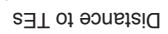

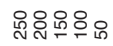

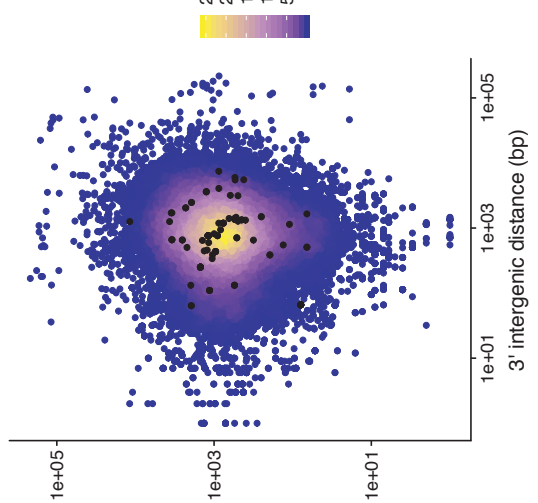

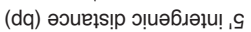
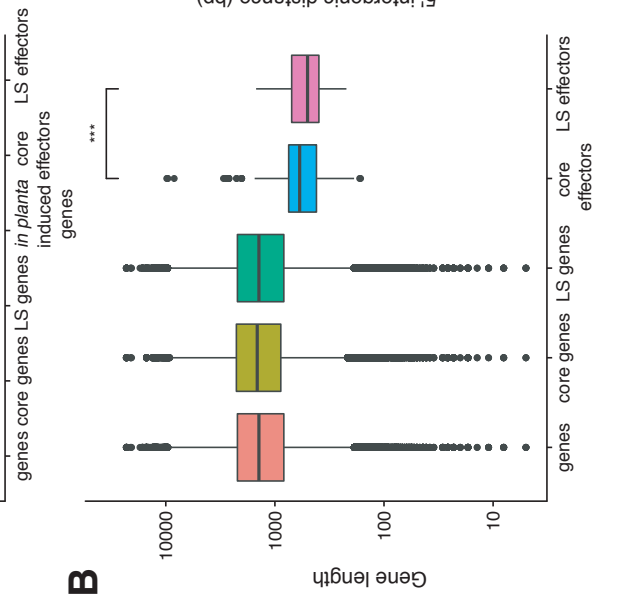

ำ

का

$\frac{1}{0}$

$\stackrel{ \pm}{\subseteq}$

을

등

茫

in

음 늠

in 은

प은

응 웡 증

入 $\frac{2}{0}$

등

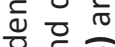

$\frac{\sigma}{\sigma} \frac{\tilde{\sigma}}{\sigma}$

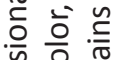

व 0

छे

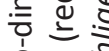

이동

¿ ㅇ

岳 导

당

ब워

啘

v 드

ฮั

क्ष

ㅎํㄴ 을

늘

幽

ง

을 능

บㄴ

ํํㅇ ํํำ

4 ㄴํㄴ

บ넝

号出先

는 01

t)

인 논

它 $\frac{1}{3}$

ต ज

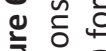

흠 음 
Thus, we searched the 333 LS effector genes against the $V$. dahliae genome assemblies using BLAST (tblastn), which revealed that multiple (candidate) effector genes, including the previous identified ones, were absent from the gene annotation, highlighting the challenge of computational effector gene identification (Gibriel et al. 2016). Intriguingly, we observed highly dissimilar LS effector catalogs among $V$. dahliae strains that are able to infect the same host plant (Figure 8). Of the 333 LS effectors, not even a single one is shared among all strains that infect the same host plant (Figure 8). Even more strikingly, $V$. dahliae strains that infect the same host plant do not cluster based on their LS effector repertoires (Figure 8). Thus, $V$. dahliae strains harbor highly divergent LS effector catalogs, the composition of which does not correlate with the host plant they are able to infect.

\section{Variability in core effector transcription profiles}

In many plant pathogens, core effector genes are in planta highly-induced and play essential roles on a multitude of hosts (Guyon et al. 2014; Hemetsberger et al. 2015; Santhanam et al. 2013; Yin et al. 2017). Thus, we assessed the expression of V. dahliae core effector genes during invasion of different plant species. First, we mapped RNAseq datasets from $N$. benthamiana plants colonized by $V$. dahliae strain JR2 (de Jonge et al. 2012) against the reference genome sequence of $V$. dahliae strain JR2 (Faino et al. 2015), and G. hirsutum (cotton) plants colonized by V. dahliae strain V991 (LF Zhu, unpublished data) against its closely related $V$. dahliae strain CQ2. Subsequently, RNAseq reads overlapping shared core effector genes of $V$. dahliae JR2 and $V$. dahliae strain CQ2 were quantified. We observed that the transcription profiles can be clustered into: 1) transcribed effector genes on both hosts (those with log10 TPM value $>0$ in both hosts), 2) differentially transcribed effector genes between the two hosts (those with log10 TPM value of 0 in one host and $>0$ in the other host), and 3) non-transcribed effector genes on either hosts (those with TPM value of 0 in both hosts) (Figure 9A). Of the 165 shared core effector genes between JR2 and CQ2, 61 effector genes were transcribed during cotton as well as $N$. benthamiana colonization, whereas 19 effector genes were only transcribed on cotton, and 41 effector genes were transcribed on $N$. benthamiana (Figure 9B). Additionally, we identified 44 effector genes that were non-transcribed in both strains (Figure 9A). Thus, differential $V$. dahliae core effector gene expression is observed on different host plants. Overall, we are not able to link the composition or the expression of effector gene catalogs to the ability to infect particular host plants. 

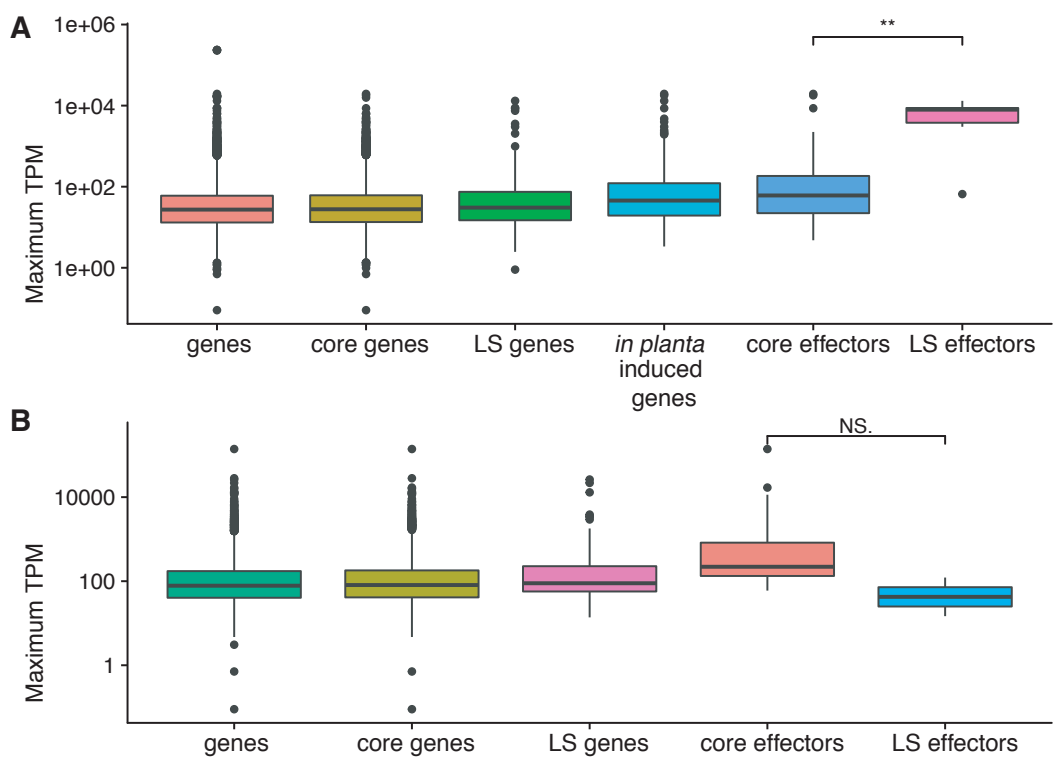

Figure 7. Maximum TPM values of core and LS effector genes over four time points during N. benthamiana and cotton infection by V. dahliae strains JR2 and V911. A) Maximum TPM values per time point $(4,8,12$, and 16$)$ of core and LS effector genes during $N$. benthamiana infection by $V$. dahliae strain JR2. B) Maximum TPM values per time point (6, 9, 12, and 15) of core and LS effector genes during cotton infection by $V$. dahliae strain V911. 
Figure 8. Verticillium dahliae strains that infect the same host plant harbor highly divergent LS effector repertoires. LS effectors were clustered into 110 families and their presence (brown) and absence (white) between $V$. dahliae strains is shown. Previously described LS effectors are colored in red (de Jonge et al. 2013; Chapter 4). Black colors indicate the absence of LS effectors from the predicted gene annotation for $V$. dahliae strains and the presence in $V$. dahliae genomes based on BLAST (tblastn).

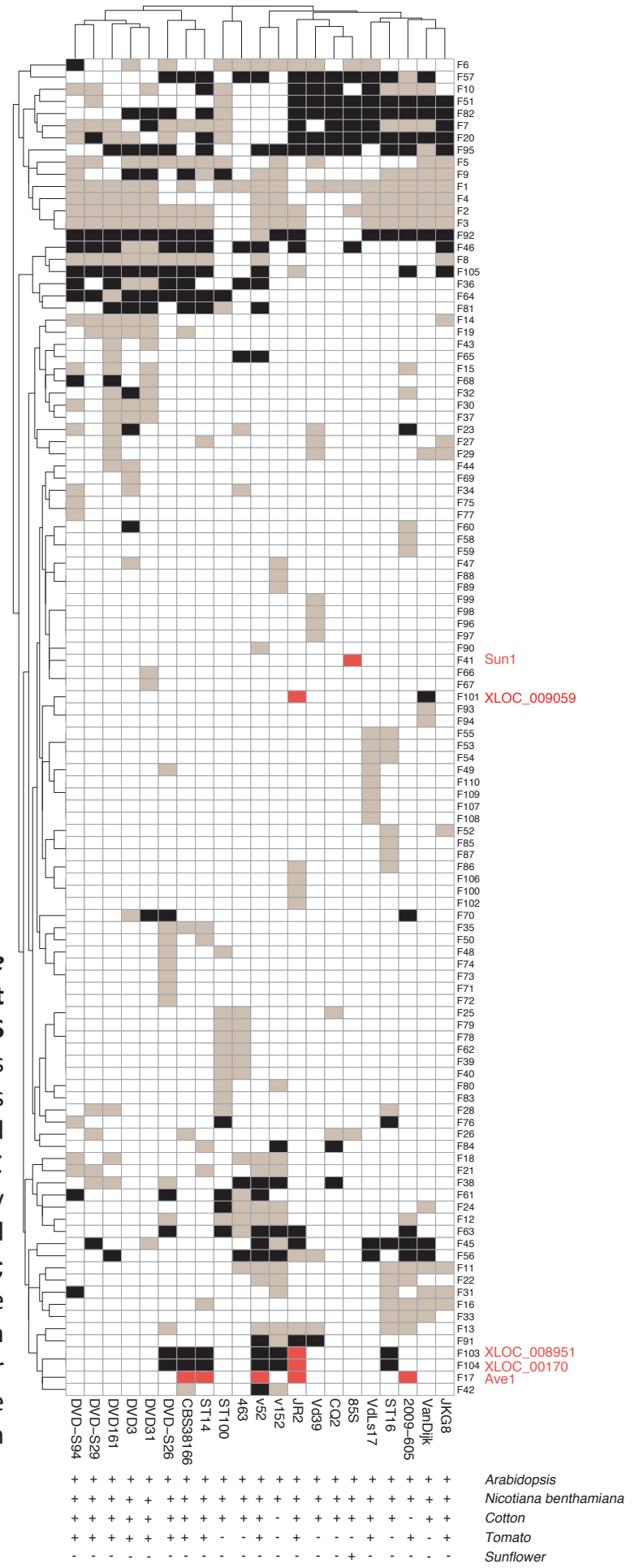


A

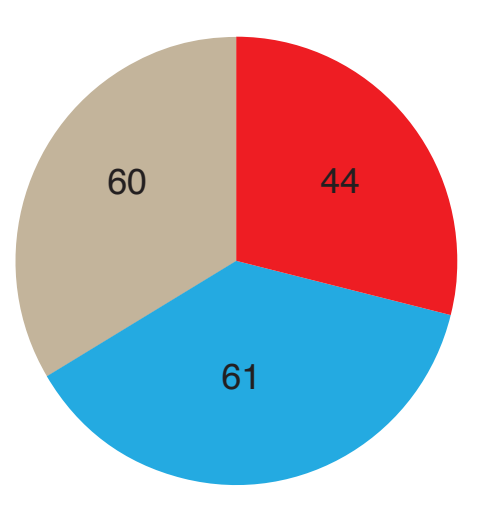

Non-transcribed on two hosts

Transcribed on two hosts

Differentially transcribed
B

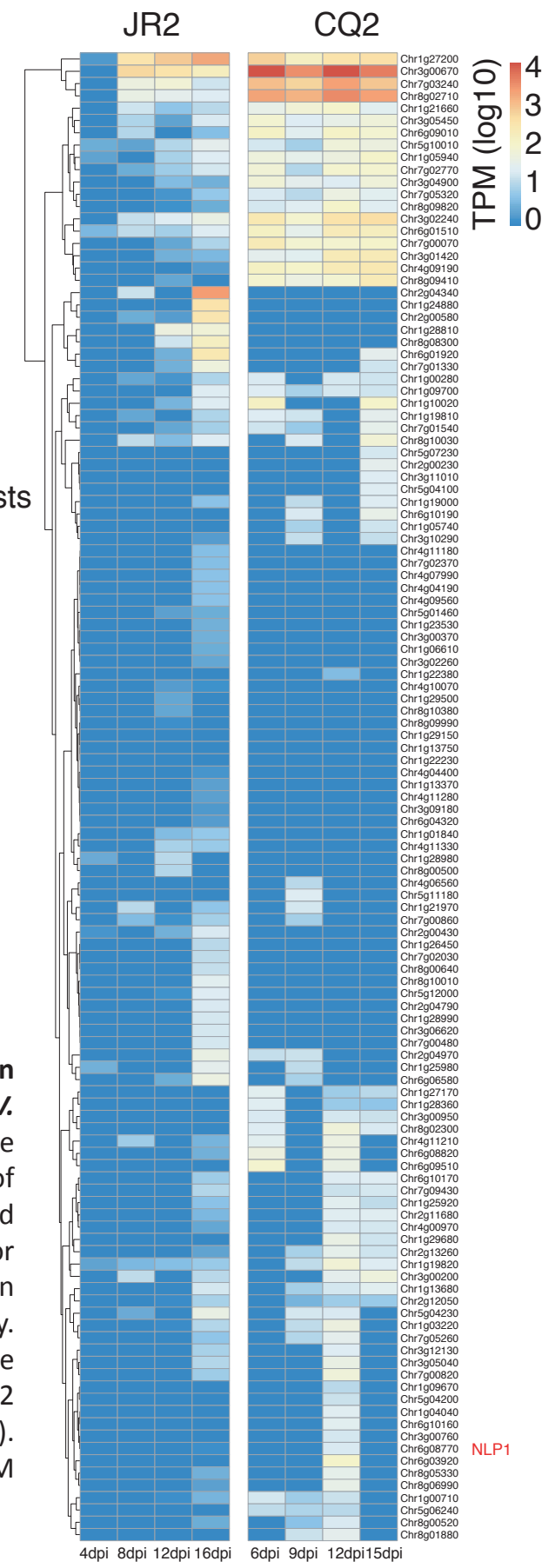

Figure 9. Variability in transcription profiles of core effector genes of $V$. dahliae strains JR2 and CQ2. A) Pie chart summarizing the number of non-transcribed, transcribed, and differentially transcribed core effector genes of $V$. dahliae strain JR2 and CQ2 on $N$. benthamiana and cotton, respectively. B) Heatmap of the TPM values of core effector genes of $V$. dahliae strain JR2 (left) and $V$. dahliae strain CQ2 (right). The color gradient shows log-scaled TPM values. 


\section{DISCUSSION}

For broad host-range pathogens such as $V$. dahliae it is unclear to what extent coevolution of the pathogen with multiple hosts occurs simultaneously, and what implications this has for their effector repertoires. Whereas it is conceivable that broad host-range pathogens employ core effectors that are active on a multitude of hosts because they target broadly conserved general physiological processes in these hosts, they may also harbor an array of specialized effectors that only exert their activity on particular host species. Presence of such specialized effectors is suggested by the observation that different $V$. dahliae strains, despite their general ability to infect a wide array of host plants, generally display differential capacity to infect particular hosts (Bhat and Subbarao 1999). Therefore, in this study we investigated whether the absence or presence of particular effectors correlates with the ability to infect particular hosts. First, we predicted the LS effector gene repertoires of $V$. dahliae strains and identified 333 LS effector genes, of which the previously identified LS effector genes Ave1 of $V$. dahliae strain JR2 (de Jonge et al. 2013) and the Sun1 effector gene of $V$. dahliae strain 855 (Chapter 4) were successfully predicted (Figure 8). As improper gene annotation could hamper effector gene discovery (Gibriel et al. 2016), we searched the 333 effector genes against $V$. dahliae genomes using BLAST (tblastn) and successfully identified the previously described effector genes XLOC_00170, XLOC_008951, and XLOC_009059 of $V$. dahliae strain JR2, which are shared by a subset of $V$. dahliae strains (de Jonge et al. 2013) (Figure 8). Intriguingly, we observed that $V$. dahliae strains that are able to infect the same host plant, in this case focused on tomato, cotton, $N$. benthamiana, Arabidopsis, and sunflower, harbor highly divergent LS effector catalogs and not even a single LS effector is shared by the strains that are able to infect either of these host species (Figure 8). This strongly suggests that different strains infect the same host plant by utilizing different effector compositions. If one assumes that different strains of $V$. dahliae must target the same host physiological processes in order to establish the infection, this implies that there must be a significant degree of redundancy among the various effectors that occur in the different strains. Interestingly, fungi of the ascomycete species Fusarium oxysporum display a similar infection biology as $V$. dahliae, being soil-borne pathogens that colonize the xylem tissues of their host plants to cause vascular wilt disease and with a largely overlapping host range (Ma et al. 2013). However, whereas individual strains of $V$. dahliae are characterized by their generally broad host range, strains of $F$. oxysporum are generally host-specific and are therefore assigned to formae speciales (Ma et al. 2013; Ma et al. 2010). Comparative genomics revealed that Fusarium strains able to infect various host plants carry highly overlapping LS effector repertoires (Coleman et al. 2009; Ma et al. 2010; van Dam et al. 2016). Moreover, strains that infect the same host plant cluster based on their LS effector repertoires (Van Dam et al. 2016). Thus, in contrast to F. oxysporum strains that harbor highly overlapping LS effectors based on their host range (Coleman et al. 2009; Ma et al. 2010; van Dam et al. 2016), V. dahliae strains evolved highly divergent LS effector catalogs, the composition of which does not correlate with the host plant they are able to infect. 
Plant pathogens harbor an array of in planta highly-induced core effector genes that play essential roles on a multitude of hosts (Guyon et al. 2014; Hemetsberger et al. 2015; Santhanam et al. 2013; Yin et al. 2017). We quantified RNAseq reads (RNAseq reads of $V$. dahliae JR2-infecting $N$. benthamiana plants and RNAseq reads of $V$. dahliae strain V991-infecting cotton) overlapping core effector genes of JR2 and CQ2 (a strains closely related to V991). Subsequently, we categorized the effectors in three groups based on their expression profiles. We identified a group of effector genes that are transcribed in both strains (Figure 9B), suggesting that this group of effectors contributes to $V$. dahliae colonization of $N$. benthamiana and cotton. Additionally, we observed a group of effector genes that are highly transcribed in $V$. dahliae strain JR2 on $N$. benthamiana but only lowly transcribed in strain CQ2 on cotton, and vice versa (Figure 9B), suggesting that they differentially contribute to virulence on these two host plants. Furthermore, we identified a group of effector genes that are not transcribed in either strains (Figure 9A), suggesting that this group of core effectors do not play a role in virulence on $N$. benthamiana or on cotton. Nevertheless, it may well be that this group of effector genes is transcribed during $V$. dahliae colonization of other host plants. Surprisingly, we observed that a member of the family of necrosis- and ethyleneinducing-like proteins (NLPs), namely NLP1, is not expressed on $N$. benthamiana and is lowly expressed on cotton (Figure 9B), whereas we previously found based on real-time PCR that this effector gene is transcribed in V. dahliae strain JR2 on N. benthamiana and on tomato, but also in V. dahliae strain V592 on cotton (Santhanam et al. 2013; Zhou et al. 2012). Likely, the low expression of NLP1 expression based on our RNAseq data may be due to the low amount of fungal RNAseq reads among plant-derived ones, as only $0.05 \%$ of the reads could be mapped to the $V$. dahliae genome (Faino et al. 2014). Thus, it needs to be taken into account that only highly expressed fungal genes are identified, and it might be worthwhile to confirm whether the group of non-transcribed effector genes still contains lowly or moderately expressed genes based on real-time PCR.

Localization of pathogen effector genes within dynamic genomic regions allows pathogens to rapidly evolve to evade plant immunity once an effector gets recognized by the host (Dong et al. 2015; Raffaele and Kamoun 2012). When we compared the core and LS effector repertoires between the analysed $V$. dahliae strains, we observed no remarkable differences in the number of core and LS effectors (Table 2). Nevertheless, by comparing features of core and LS effectors we observed that LS effectors are typically shorter in length, localize significantly closer to TEs, and have slightly longer inter-genic lengths when compared with core effectors (Figure 6). Consistent with this observation, a recent study that analysed the genomes of nine different Verticillium species showed that species-specific genes displayed significantly shorter gene lengths and longer inter-genic lengths when compared with genes that are conserved across the various species within the genus (Shi-Kunne et al. 2017). Similarly, LS genes of the fungal wheat pathogen $Z$. tritici were frequently found to be shorter, closer to TEs, and have longer inter-genic lengths, when compared with core genes (Haueisen et al. 2017; Plissonneau et al. 2018; Plissonneau et al. 2016). It has been previously suggested 
that the localization of effector genes in close proximity to TEs and within gene-poor regions mediate rapid evolution of effector catalogs (Raffaele and Kamoun 2012; Seidl and Thomma 2017). Thus, the localization of LS effector genes of $V$. dahliae strains in close proximity to TEs may mediate accelerated evolution of effector catalogs.

In conclusion, our data demonstrate the extensive variability within the effector repertoires of the broad host range pathogen $V$. dahliae. We have demonstrated that LS effectors are highly divergent among $V$. dahliae strains that infect the same host plant, and core effector genes are differentially expressed between hosts. The variability within LS and core effector genes of $V$. dahliae strains may lead to rapid immunity evasion, which may allow pathogen strains to be competitive in the co-evolution with their multiple hosts.

\section{ACKNOWLEDGEMENTS}

J Li acknowledges a PhD fellowship from China Scholarship Council (CSC). Work in the laboratories of M.F.S and B.P.H.J.T. and is supported by the Research Council Earth and Life Sciences (ALW) of the Netherlands Organization of Scientific Research (NWO). 


\section{REFERENCES}

Altschul, S. F., Gish, W., Miller, W., Myers, E. W., and Lipman, D. J. 1990. Basic local alignment search tool. Journal of Molecular Biology 215.

Apweiler, R., Bairoch, A., Wu, C. H., Barker, W. C., Boeckmann, B., Ferro, S., Gasteiger, E., Huang, H., Lopez, R., Magrane, M., Martin, M. J., Natale, D. A., O’Donovan, C., Redaschi, N., and Yeh, L.-S. L. 2004. Uniprot: the universal protein knowledgebase. Nucleic Acids Research 32:D115-D119.

Bhat, R. G., and Subbarao, K. V. 1999. Host range specificity in Verticillium dahliae. Phytopathology 89:1218-1225.

Coleman, J. J., Rounsley, S. D., Rodriguez-Carres, M., Kuo, A., Wasmann, C. C., Grimwood, J., Schmutz, J., Taga, M., White, G. J., Zhou, S., Schwartz, D. C., Freitag, M., Ma, L.-j., Danchin, E. G. J., Henrissat, B., Coutinho, P. M., Nelson, D. R., Straney, D., Napoli, C. A., Barker, B. M., Gribskov, M., Rep, M., Kroken, S., Molnár, I., Rensing, C., Kennell, J. C., Zamora, J., Farman, M. L., Selker, E. U., Salamov, A., Shapiro, H., Pangilinan, J., Lindquist, E., Lamers, C., Grigoriev, I. V., Geiser, D. M., Covert, S. F., Temporini, E., and VanEtten, H. D. 2009. The genome of Nectria haematococca: Contribution of supernumerary chromosomes to gene expansion. PLoS Genetics 5:e1000618.

Cook, D. E., Mesarich, C. H., and Thomma, B. P. H. J. 2015. Understanding plant immunity as a surveillance system to detect invasion. Annual Review of Phytopathology 53:541-563.

Croll, D., and McDonald, B. A. 2012. The accessory genome as a cradle for adaptive evolution in pathogens. PLoS Pathogens 8:e1002608.

de Jonge, R., Bolton, M. D., Kombrink, A., van den Berg, G. C. M., Yadeta, K. A., and Thomma, B. P. H. J. 2013. Extensive chromosomal reshuffling drives evolution of virulence in an asexual pathogen. Genome Research 23:1271-1282.

de Jonge, R., Bolton, M. D., and Thomma, B. P. H. J. 2011. How filamentous pathogens co-opt plants: the ins and outs of fungal effectors. Current Opinion in Plant Biology 14:400-406.

de Jonge, R., Peter van Esse, H., Maruthachalam, K., Bolton, M. D., Santhanam, P., Saber, M. K., Zhang, Z., Usami, T., Lievens, B., Subbarao, K. V., and Thomma, B. P. H. J. 2012. Tomato immune receptor Ve1 recognizes effector of multiple fungal pathogens uncovered by genome and RNA sequencing. Proceedings of the National Academy of Sciences of the United States of America 109:5110-5115.

de Jonge, R., van Esse, H. P., Kombrink, A., Shinya, T., Desaki, Y., Bours, R., van der Krol, S., Shibuya, N., Joosten, M. H., and Thomma, B. P. H. J. 2010. Conserved fungal LysM effector Ecp6 prevents chitin-triggered immunity in plants. Science 329:953-955.

Dobin, A., Davis, C. A., Schlesinger, F., Drenkow, J., Zaleski, C., Jha, S., Batut, P., Chaisson, M., and Gingeras, T. R. 2013. STAR: ultrafast universal RNA-seq aligner. Bioinformatics 29:15-21.

Dong, S., Raffaele, S., and Kamoun, S. 2015. The two-speed genomes of filamentous pathogens: waltz with plants. Current Opinion in Genetics \& Development 35:5765. 
Faino, L., de Jonge, R., and Thomma, B. P. H. J. 2014. The transcriptome of Verticillium dahliae-infected Nicotiana benthamiana determined by deep RNA sequencing. Plant Signaling \& Behavior 7:1065-1069.

Faino L, Seidl MF, Datema E, van den Berg GC, Janssen A, Wittenberg AH, Thomma B. P. H. J. 2015. Single-molecule real-time sequencing combined with optical mapping yields completely finished fungal genome. mBio 6: e00936-00915.

Fisher, M. C., Henk, D. A., Briggs, C. J., Brownstein, J. S., Madoff, L. C., McCraw, S. L., and Gurr, S. J. 2012. Emerging fungal threats to animal, plant and ecosystem health. Nature 484:186-194.

Fradin, E. F., and Thomma, B. P. H. J. 2006. Physiology and molecular aspects of Verticillium wilt diseases caused by $V$. dahliae and $V$. albo-atrum. Molecular Plant Pathology 7:71-86.

Fradin, E. F., Zhang, Z., Juarez Ayala, J. C., Castroverde, C. D. M., Nazar, R. N., Robb, J., Liu, C.-M., and Thomma, B. P. H. J. 2009. Genetic dissection of Verticillium wilt resistance mediated by tomato Ve1. Plant Physiology 150:320-332.

Gibriel, H. A. Y., Thomma, B. P. H. J., and Seidl, M. F. 2016. The age of effectors: genomebased discovery and applications. Phytopathology 106: 1206-1212.

Gurevich, A., Saveliev, V., Vyahhi, N., and Tesler, G. 2013. QUAST: quality assessment tool for genome assemblies. Bioinformatics 29:1072-1075.

Guyon, K., Balagué, C., Roby, D., and Raffaele, S. 2014. Secretome analysis reveals effector candidates associated with broad host range necrotrophy in the fungal plant pathogen Sclerotinia sclerotiorum. BMC Genomics 15:336.

Haueisen, J., Moeller, M., Eschenbrenner, C. J., Grandaubert, J., Seybold, H., Adamiak, H., and Stukenbrock, E. H. 2017. Extremely flexible infection programs in a fungal plant pathogen. bioRxiv: https://doi.org/10.1101/229997.

Hemetsberger, C., Mueller, A. N., Matei, A., Herrberger, C., Hensel, G., Kumlehn, J., Mishra, B., Sharma, R., Thines, M., Hückelhoven, R., and Doehlemann, G. 2015. The fungal core effector Pep1 is conserved across smuts of dicots and monocots. New Phytologist 206:1116-1126.

Holt, C., and Yandell, M. 2011. MAKER2: an annotation pipeline and genome-database management tool for second-generation genome projects. BMC Bioinformatics 12:491.

Inderbitzin, P., Bostock, R. M., Davis, R. M., Usami, T., Platt, H. W., and Subbarao, K. V. 2011. Phylogenetics and taxonomy of the fungal vascular wilt pathogen Verticillium, with the descriptions of five new species. PLoS One 6:e28341.

Jones, J. D. G., and Dangl, J. L. 2006. The plant immune system. Nature 444:323-329.

Kema, G. H. J., Mirzadi Gohari, A., Aouini, L., Gibriel, H. A. Y., Ware, S. B., van den Bosch, F., Manning-Smith, R., Alonso-Chavez, V., Helps, J., Ben M'Barek, S., Mehrabi, R., Diaz-Trujillo, C., Zamani, E., Schouten, H. J., van der Lee, T. A. J., Waalwijk, C., de Waard, M. A., De Wit, P. J. G. M., Verstappen, E. C. P., Thomma, B. P. H. J., Meijer, H. J. G., and Seidl, M. F. 2018. Stress and sexual reproduction affect the dynamics of the wheat pathogen effector AvrStb6 and strobilurin resistance. Nature Genetics 23:678. 
Kolde, R. 2015. pheatmap: Pretty Heatmaps. R package version 1.0. 8.

Kombrink, A., Rovenich, H., Shi-Kunne, X., Rojas-Padilla, E., van den Berg, G. C. M., Domazakis, E., de Jonge, R., Valkenburg, D.-J., Sánchez-Vallet, A., Seidl, M. F., and Thomma, B. P. H. J. 2017. Verticillium dahliae LysM effectors differentially contribute to virulence on plant hosts. Molecular Plant Pathology 18:596-608.

Korf, I. 2004. Gene finding in novel genomes. BMC Bioinformatics 5: 59.

Kurtz, S., Phillippy, A., Delcher, A. L., Smoot, M., Shumway, M., Antonescu, C., and Salzberg, S. L. 2004. Versatile and open software for comparing large genomes. Genome Biology 5: R12.

Li, B., and Dewey, C. N. 2011. RSEM: accurate transcript quantification from RNA-Seq data with or without a reference genome. BMC Bioinformatics 12:323.

Li, L., Stoeckert, C. J., and Roos, D. S. 2003. OrthoMCL: identification of ortholog groups for eukaryotic genomes. Genome Research 13:2178-2189.

Lukashin, A. V., and Borodovsky, M. 1998. GeneMark.hmm: new solutions for gene finding. Nucleic Acids Research 26.

Ma, L.-J., Geiser, D. M., Proctor, R. H., Rooney, A. P., O’Donnell, K., Trail, F., Gardiner, D. M., Manners, J. M., and Kazan, K. 2013. Fusarium pathogenomics. Annual Review of Microbiology 67:399-416.

Ma, L. J., Does, H. C., Borkovich, K. A., Coleman, J. J., Daboussi, M. J., Pietro, A., Dufresne, M., Freitag, M., Grabherr, M., and Henrissat, B. 2010. Comparative genomics reveals mobile pathogenicity chromosomes in Fusarium. Nature 464.

Marshall, R., Kombrink, A., Motteram, J., Loza-Reyes, E., Lucas, J., Hammond-Kosack, K. E., Thomma, B. P. H. J., and Rudd, J. J. 2011. Analysis of two in planta expressed LysM effector homologs from the fungus Mycosphaerella graminicola reveals novel functional properties and varying contributions to virulence on wheat. Plant Physiology 156:756-769.

Meile, L., Croll, D., Brunner, P. C., Plissonneau, C., Hartmann, F. E., McDonald, B. A., and Sánchez-Vallet, A. 2018. A fungal avirulence factor encoded in a highly plastic genomic region triggers partial resistance to septoria tritici blotch. New Phytologist 65:512.

Mentlak, T. A., Kombrink, A., Shinya, T., Ryder, L. S., Otomo, I., Saitoh, H., Terauchi, R., Nishizawa, Y., Shibuya, N., Thomma, B. P. H. J., and Talbot, N. J. 2012. Effectormediated suppression of chitin-triggered immunity by Magnaporthe oryzae is necessary for rice blast disease. The Plant Cell 24:322-335.

Pennisi, E. 2010. Armed and dangerous. Science 327:804-805.

Petersen, T. N., Brunak, S., von Heijne, G., and Nielsen, H. 2011. SignalP 4.0: discriminating signal peptides from transmembrane regions. Nature Methods 8:785-786.

Plissonneau, C., Hartmann, F. E., and Croll, D. 2018. Pangenome analyses of the wheat pathogen Zymoseptoria tritici reveal the structural basis of a highly plastic eukaryotic genome. BMC Biology 16:673.

Plissonneau, C., Stürchler, A., and Croll, D. 2016. The evolution of orphan regions in genomes of a fungal pathogen of wheat. mBio 7. 
Quinlan, A. R., and Hall, I. M. 2010. BEDTools: a flexible suite of utilities for comparing genomic features. Bioinformatics 26.

Raffaele, S., and Kamoun, S. 2012. Genome evolution in filamentous plant pathogens: why bigger can be better. Nature Review Microbiology 10:417-430.

Santhanam, P., van Esse, H. P., Albert, I., Faino, L., Nürnberger, T., and Thomma, B. P. H. J. 2013. Evidence for functional diversification within a fungal NEP1-like protein family. Molecular Plant Microbe Interactions 26:278-286.

Schmidt, S. M., Houterman, P. M., Schreiver, I., Ma, L., Amyotte, S., Chellappan, B., Boeren, S., Takken, F. L. W., and Rep, M. 2013. MITEs in the promoters of effector genes allow prediction of novel virulence genes in Fusarium oxysporum. BMC Genomics 14:1-21.

Seidl, M. F., and Thomma, B. 2017a. Transposable elements direct the coevolution between plants and microbes. Trends in Genetics 33:842-851.

Shi-Kunne, X., Faino, L., van den Berg, G. C. M., Thomma, B. P. H. J., and Seidl, M. F. 2017. Evolution within the fungal genus Verticillium is characterized by chromosomal rearrangement and gene loss. Environmental Microbiology 20:1362-1373.

Simão, F. A., Waterhouse, R. M., loannidis, P., Kriventseva, E. V., and Zdobnov, E. M. 2015. BUSCO: assessing genome assembly and annotation completeness with single-copy orthologs. Bioinformatics 31:3210-3212.

Smit, A., and Hubley, R. 2010. RepeatModeler Open-1.0. Repeat Masker Website.

http://www.repeatmasker.org/Repeat-Modeler.html.

Smit, A., Hubley, R., and Green, P. 2016. RepeatMasker Open-4.0. Available from: http:// www.repeatmasker.org

Song, Y., Liu, L., Wang, Y., Valkenburg, D.-J., Zhang, X., Zhu, L., and Thomma, B. P. H. J. 2017. Transfer of tomato immune receptor Ve1 confers Ave1-dependent Verticillium resistance in tobacco and cotton. Plant Biotechnology Journal 16:638-648.

Sperschneider, J., Gardiner, D. M., Dodds, P. N., Tini, F., Covarelli, L., Singh, K. B., Manners, J. M., and Taylor, J. M. 2016. EffectorP: predicting fungal effector proteins from secretomes using machine learning. New Phytologist 210:743-761.

Stanke, M., and Waack, S. 2003. Gene prediction with a hidden Markov model and a new intron submodel. Bioinformatics 19.

Stergiopoulos, I., van den Burg, H. A., Okmen, B., Beenen, H. G., van Liere, S., Kema, G. H., and de Wit, P. J. 2010. Tomato Cf resistance proteins mediate recognition of cognate homologous effectors from fungi pathogenic on dicots and monocots. Proceedings of the National Academy of Sciences of the United States of America 107:7610-7615.

Takahara, H., Hacquard, S., Kombrink, A., Hughes, H. B., Halder, V., Robin, G. P., Hiruma, K., Neumann, U., Shinya, T., Kombrink, E., Shibuya, N., Thomma, B. P. H. J., and O'Connell, R. J. 2016. Colletotrichum higginsianum extracellular LysM proteins play dual roles in appressorial function and suppression of chitin-triggered plant immunity. New Phytologist 211:1323-1337.

Tritt, A., Eisen, J. A., Facciotti, M. T., and Darling, A. E. 2012. An integrated pipeline for de novo assembly of microbial genomes. PLoS One 7:e42304. 
van Dam, P., Fokkens, L., Schmidt, S. M., Linmans, J. H. J., Kistler, H. C., Ma, L.-J., and Rep, M. 2016. Effector profiles distinguish formae speciales of Fusarium oxysporum. Environmental Microbiology 18:4087-4102.

Yin, J., Gu, B., Huang, G., Tian, Y., Quan, J., Lindqvist-Kreuze, H., and Shan, W. 2017. Conserved RXLR effector genes of Phytophthora infestans expressed at the early stage of potato infection are suppressive to host defense. Frontiers in Plant Sciences 8.

Zhou, B. J., Jia, P. S., Gao, F., and Guo, H. S. 2012. Molecular characterization and functional analysis of a necrosis- and ethylene-inducing, protein-encoding gene family from Verticillium dahliae. Molecular Plant-Microbe Interactions 25:964-975. 


\section{SUPPLEMENTAL INFORMATION}

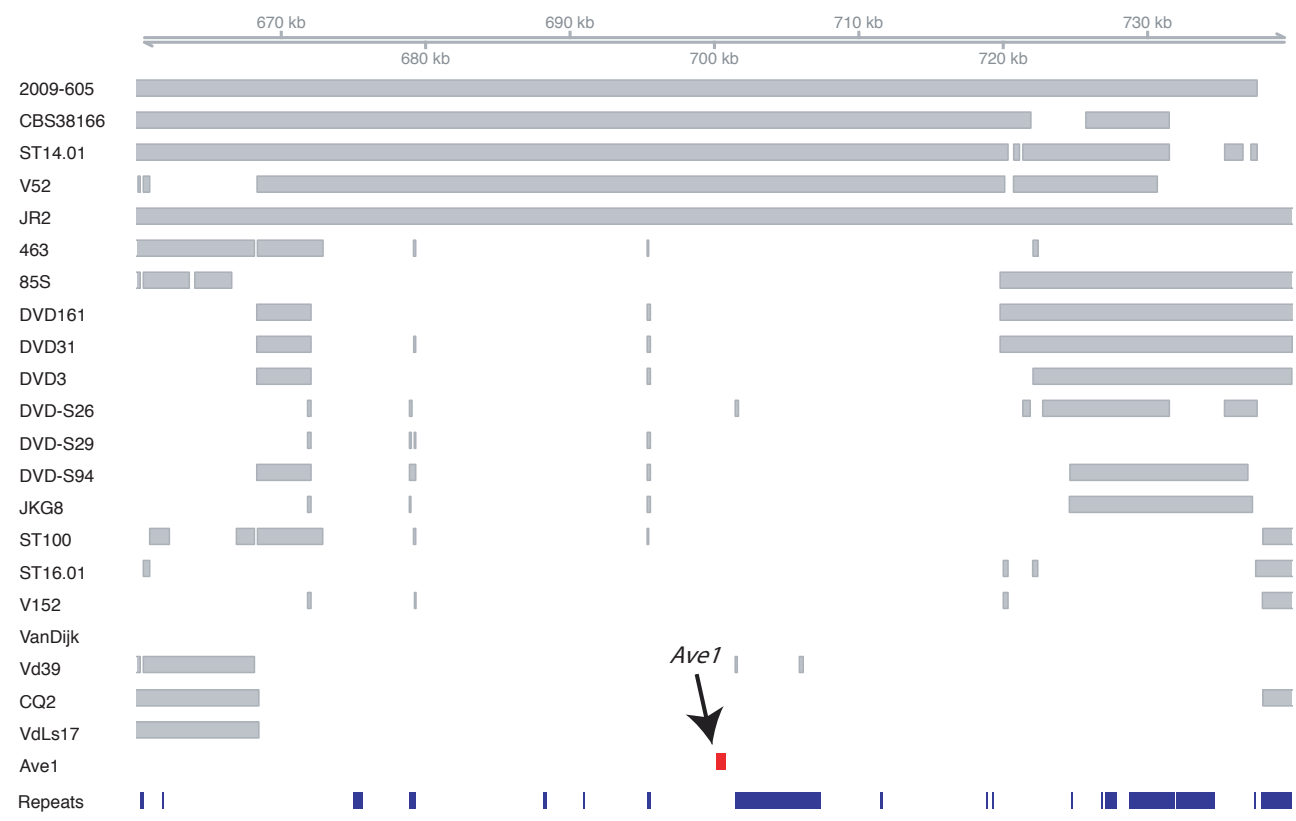

Figure S1. V. dahliae effector gene Ave1 is localized within a lineage-specific region. A highly variable, repeat-rich (blue), lineage-specific (LS) region, which harbors the Ave1 effector gene (red) of $V$. dahliae strain JR2 is shown. This LS effector gene is only present in a subset of $V$. dahliae strains. Grey bars indicate the genome alignments of $V$. dahliae strains to the reference strain JR2. This effector gene is only present in strains 2009-605, CBS38166, ST14.01, V52, and JR2, but absent in the other strains. 

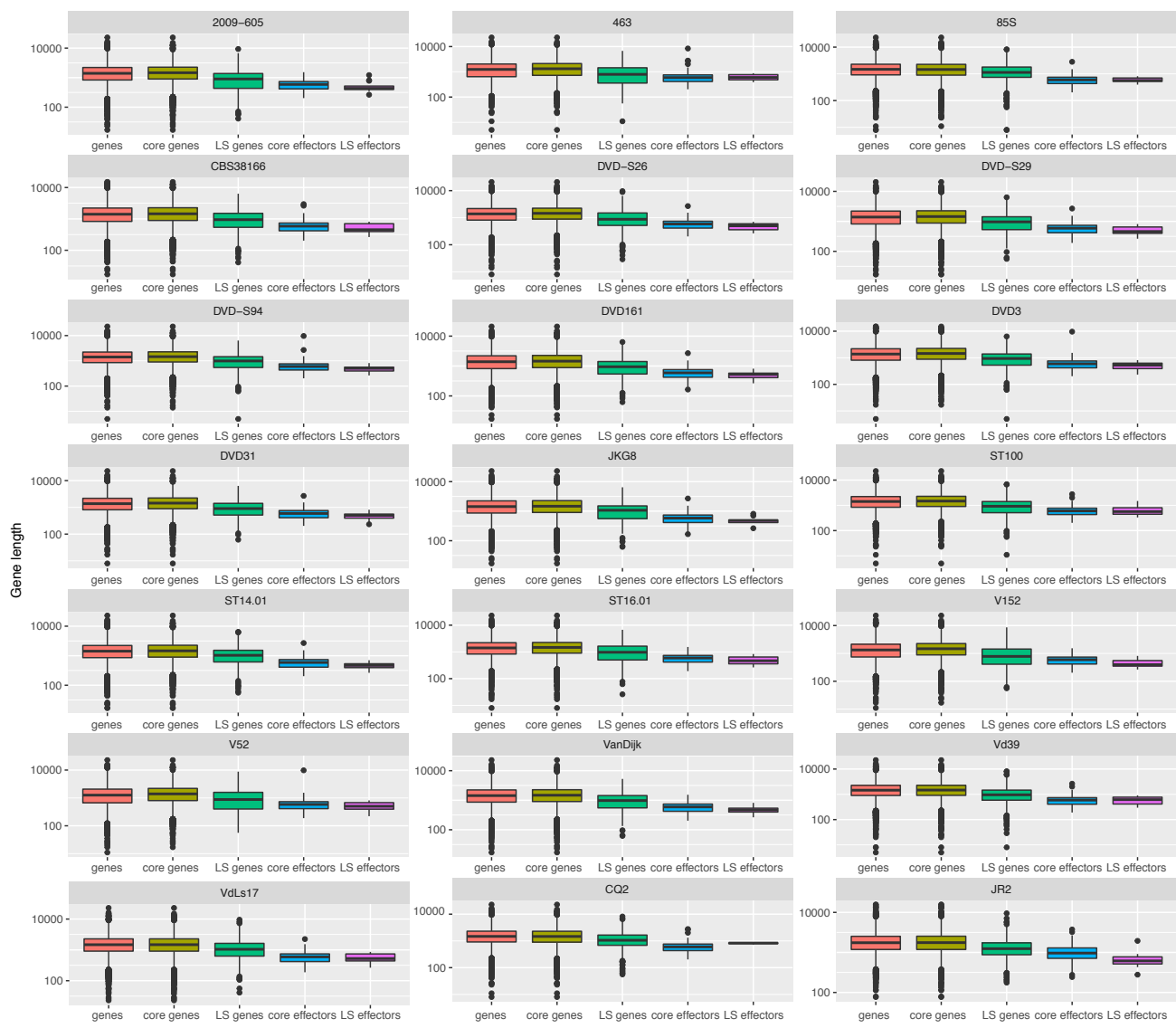

Figure S2. Length of genes and effector genes of $\boldsymbol{V}$. dahliae strains. Gene length $(\log 10)$ is shown for all genes, core genes, LS genes, core effectors, and LS effectors for all $V$. dahliae strains. 


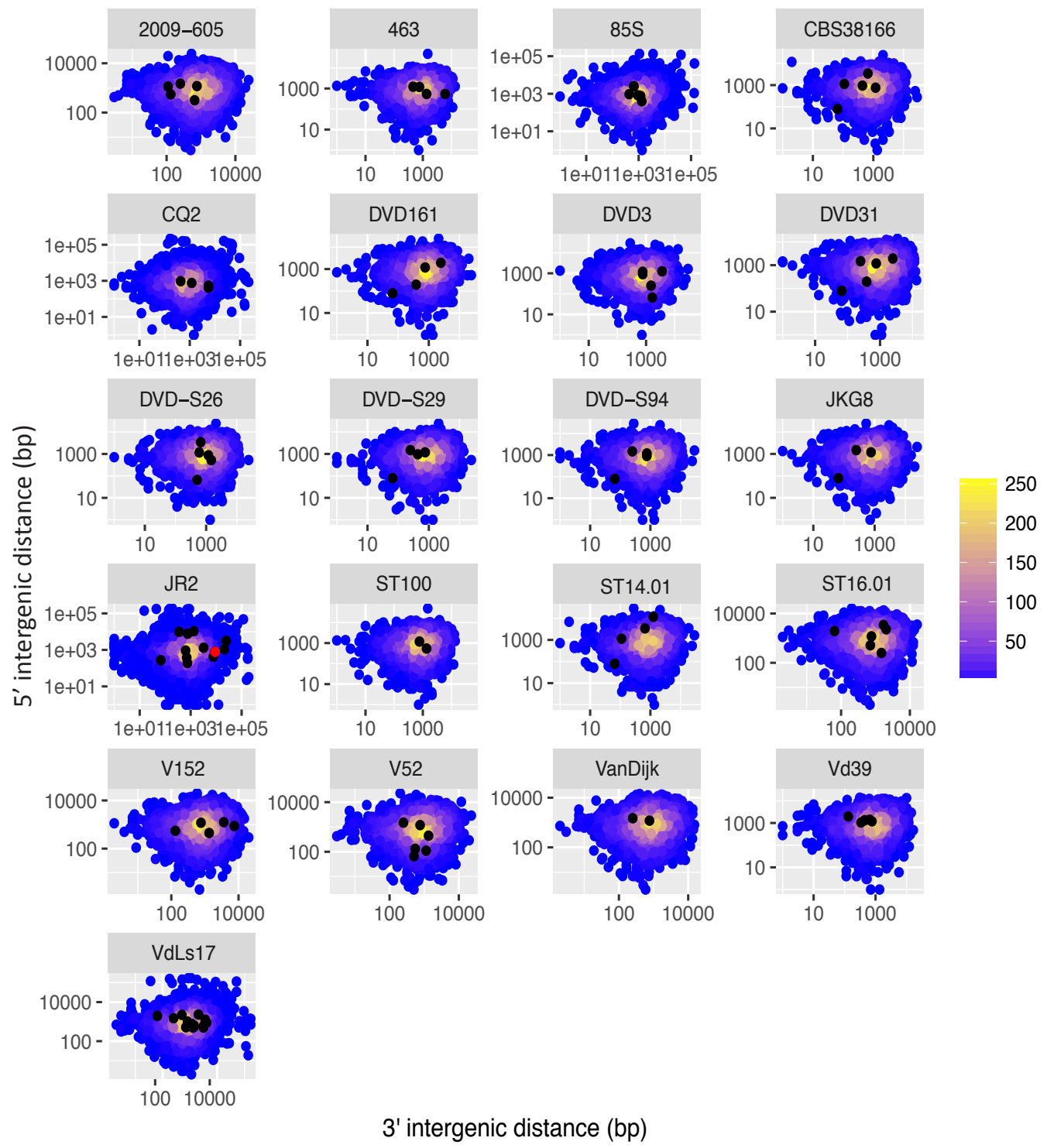

Figure S3. $\mathbf{5}^{\prime}$ and $\mathbf{3}^{\prime}$ inter-genic length of genes and effector genes of $\boldsymbol{V}$. dahliae strains. Inter-genic length (bp) is shown for all genes (blue) and LS effector genes (black) for all $V$. dahliae strains. Ave1 effector gene of $V$. dahliae strain JR2 is highlighted in red. 


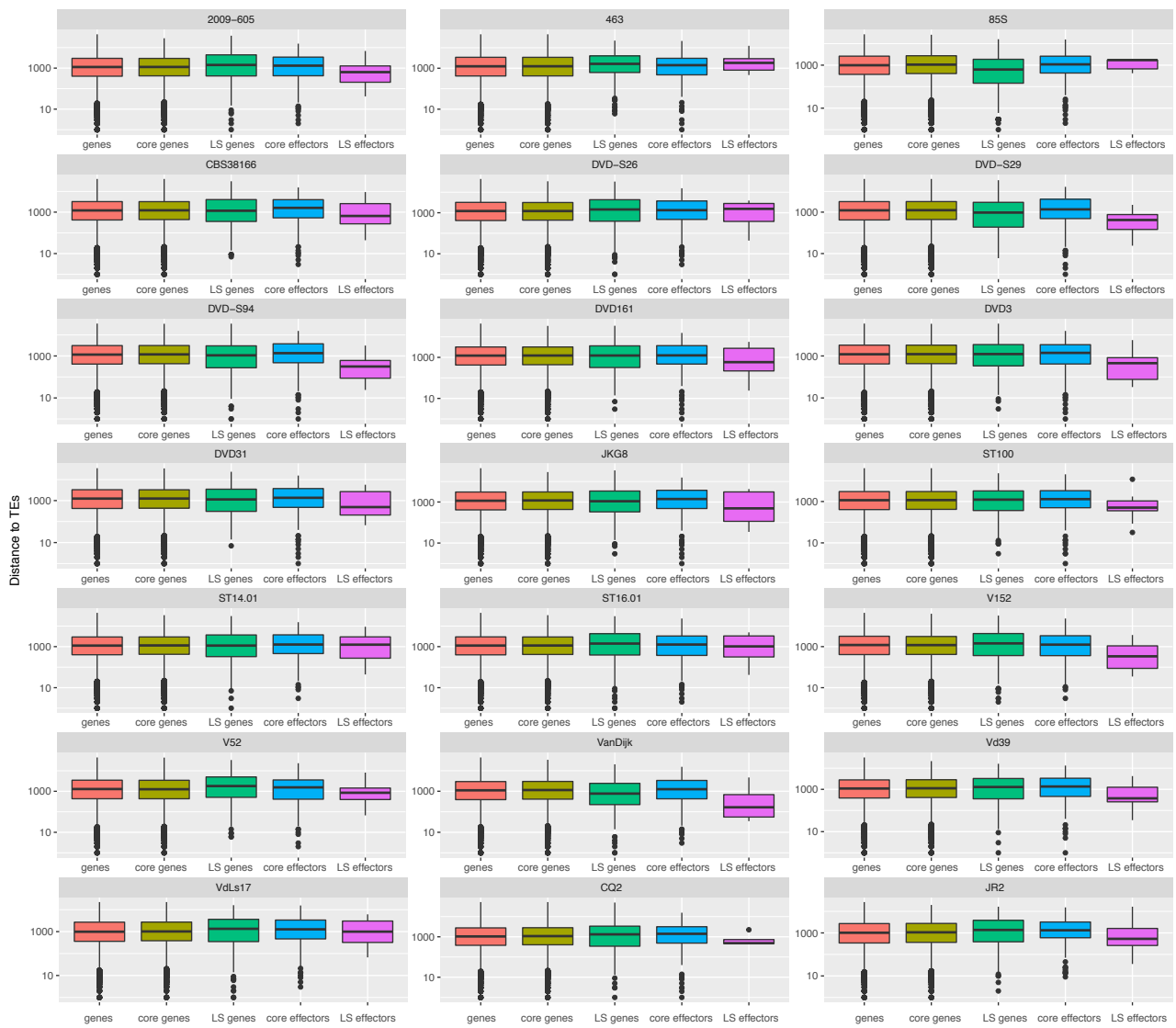

Figure S4. Distance to TEs of genes and effector gnenes of $\boldsymbol{V}$. dahliae strains. Distance to TEs ( $\log 10)$ is shown for all genes, core genes, LS genes, core effectors, and LS effectors for all $V$. dahliae strains. 
Table S1. Verticillium dahliae strains used in this study.

\begin{tabular}{|c|c|c|c|c|}
\hline Strain & $\begin{array}{l}\text { Sequencing } \\
\text { platform }\end{array}$ & Reference & Originating host & $\begin{array}{l}\text { Geographical } \\
\text { location }\end{array}$ \\
\hline CQ2 & PacBio & $\begin{array}{c}\text { (Depotter et al. } \\
2018 \text { ) }\end{array}$ & Cotton & China \\
\hline $85 S$ & PacBio & $\begin{array}{c}\text { (Depotter et al. } \\
\text { 2018) }\end{array}$ & Sunflower & France \\
\hline VdLs17 & PacBio & (Faino et al. 2015) & Lettuce & Ca, USA \\
\hline $\mathrm{JR} 2$ & PacBio & (Faino et al. 2015) & Tomato & ON, Canada \\
\hline CBS38166 & $\begin{array}{c}\text { Illumina HiSeq } \\
2000\end{array}$ & $\begin{array}{c}\text { (de Jonge et al. } \\
\text { 2012) }\end{array}$ & Tomato & QC, Canada \\
\hline ST14.01 & $\begin{array}{l}\text { Illumina HiSeq } \\
2000\end{array}$ & $\begin{array}{l}\text { (de Jonge et al. } \\
2012 \text { ) }\end{array}$ & Pistachio & CA, USA \\
\hline ST100 & $\begin{array}{c}\text { Illumina HiSeq } \\
2000\end{array}$ & $\begin{array}{c}\text { (de Jonge et al. } \\
2013 \text { ) }\end{array}$ & Soil & Belgium \\
\hline DVD3 & $\begin{array}{c}\text { Illumina HiSeq } \\
2000\end{array}$ & $\begin{array}{l}\text { (de Jonge et al. } \\
2012 \text { ) }\end{array}$ & Potato & Canada \\
\hline DVD31 & $\begin{array}{c}\text { Illumina HiSeq } \\
2000\end{array}$ & $\begin{array}{l}\text { (de Jonge et al. } \\
2012 \text { ) }\end{array}$ & Tomato & Canada \\
\hline DVD161 & $\begin{array}{c}\text { Illumina HiSeq } \\
2000\end{array}$ & $\begin{array}{c}\text { (de Jonge et al. } \\
2012 \text { ) }\end{array}$ & Potato & ON, Canada \\
\hline DVD-S26 & $\begin{array}{c}\text { Illumina HiSeq } \\
2000\end{array}$ & $\begin{array}{l}\text { (de Jonge et al. } \\
2012 \text { ) }\end{array}$ & Soil & Canada \\
\hline DVD-S29 & $\begin{array}{c}\text { Illumina HiSeq } \\
2000\end{array}$ & $\begin{array}{c}\text { (de Jonge et al. } \\
2012 \text { ) }\end{array}$ & Soil & Canada \\
\hline DVD-S94 & $\begin{array}{c}\text { Illumina HiSeq } \\
2000\end{array}$ & $\begin{array}{c}\text { (de Jonge et al. } \\
2012 \text { ) }\end{array}$ & Soil & Canada \\
\hline JKG8 & $\begin{array}{c}\text { Illumina HiSeq } \\
2000\end{array}$ & This study & Potato & The Netherlands \\
\hline $2009-605$ & $\begin{array}{c}\text { Illumina HiSeq } \\
2000\end{array}$ & This study & Bell pepper & Ukraine \\
\hline 463 & $\begin{array}{c}\text { Illumina HiSeq } \\
2000\end{array}$ & This study & Cotton & Mexico \\
\hline ST16.01 & $\begin{array}{c}\text { Illumina HiSeq } \\
2000\end{array}$ & This study & Cotton & Syria \\
\hline V152 & $\begin{array}{c}\text { Illumina HiSeq } \\
2000\end{array}$ & This study & Oak & Hungry \\
\hline V52 & $\begin{array}{c}\text { Illumina HiSeq } \\
2000\end{array}$ & This study & Pepper & Austria \\
\hline Vd39 & $\begin{array}{c}\text { Illumina HiSeq } \\
2000\end{array}$ & This study & Sunflower & Germany \\
\hline VanDijk & $\begin{array}{c}\text { Illumina HiSeq } \\
2000\end{array}$ & This study & Chrysanthemum & The Netherlands \\
\hline
\end{tabular}


Table S2. Assembly statistics for the various Verticillium dahliae genomes.

\begin{tabular}{llllllll}
\hline Strain & $\begin{array}{l}\text { Genome } \\
\text { size (Mb) }\end{array}$ & $\begin{array}{l}\text { \# scaffolds } \\
(>=0 \text { bp) }\end{array}$ & $\begin{array}{l}\text { \# scaffolds } \\
(>=1000 \mathrm{bp})\end{array}$ & $\begin{array}{l}\text { GC }(\%) \\
\text { (K50 })\end{array}$ & $\begin{array}{l}\text { \# N's per } \\
100 \mathrm{kbp}\end{array}$ & $\begin{array}{l}\text { BUSCO } \\
(\%)\end{array}$ \\
\hline 2009-605 & 34.06 & 1931 & 1521 & 54.76 & 55.11 & 123.45 & 95.4 \\
463 & 34.03 & 4188 & 3562 & 53.47 & 17.74 & 244.06 & 75 \\
85S & 35.93 & 40 & 40 & 53.55 & 3176.09 & 0 & 99.3 \\
CBS38166 & 34.03 & 2092 & 1727 & 54.24 & 45.08 & 316.06 & 91.6 \\
DVD161 & 33.47 & 2155 & 1819 & 54.4 & 41.42 & 330.79 & 90.8 \\
DVD31 & 33.58 & 2429 & 2064 & 54.14 & 35.75 & 336.71 & 89.2 \\
DVD3 & 34.42 & 1921 & 1693 & 53.62 & 42.77 & 378.65 & 88.9 \\
DVD-S26 & 34.74 & 2275 & 1894 & 54.2 & 43.54 & 367.67 & 92.2 \\
DVD-S29 & 33.14 & 2226 & 1811 & 54.56 & 42.73 & 306.89 & 91.6 \\
DVD-S94 & 34.42 & 1730 & 1494 & 53.92 & 53.17 & 287 & 91.9 \\
JKG8 & 33.85 & 1840 & 1458 & 54.5 & 56.35 & 172.12 & 95.6 \\
ST100 & 34.92 & 2137 & 1756 & 53.63 & 49.89 & 315.39 & 93 \\
ST14.01 & 34.48 & 1571 & 1336 & 54.03 & 61.81 & 221.84 & 95 \\
ST16.01 & 34.22 & 1821 & 1454 & 54.9 & 57.46 & 121.71 & 95.6 \\
V152 & 33.98 & 2539 & 2174 & 54.28 & 32.47 & 146.98 & 87.6 \\
V52 & 33.54 & 3419 & 2920 & 54.39 & 21.80 & 162.37 & 78.6 \\
VanDijk & 33.17 & 1769 & 1389 & 54.71 & 61.05 & 109.26 & 95.7 \\
Vd39 & 35.90 & 1579 & 1222 & 53.55 & 94.44 & 222.52 & 98.7 \\
CQ2 & 35.82 & 17 & 17 & 53.26 & 3754.19 & 0 & 97.5 \\
JR2 & 36.15 & 8 & 8 & 53.89 & 4168.63 & 0 & 99.4 \\
VdLs17 & 35.97 & 8 & 8 & 54 & 5894.01 & 0 & 98.9 \\
\hline
\end{tabular}


Table S3. Summary of transposable elements of the various Verticillium dahliae strains.

\begin{tabular}{|c|c|c|c|c|c|c|}
\hline \multirow[b]{2}{*}{ Strain } & \multicolumn{2}{|c|}{ SINES } & \multicolumn{2}{|c|}{ LINES } & \multicolumn{2}{|c|}{ LTRs } \\
\hline & $\begin{array}{l}\text { length } \\
\text { occupied } \\
\text { (bb) }\end{array}$ & $\begin{array}{l}\text { percentage } \\
\text { of sequence } \\
(\%)\end{array}$ & $\begin{array}{l}\text { length } \\
\text { occupied } \\
\text { (bb) }\end{array}$ & $\begin{array}{l}\text { percentage } \\
\text { of sequence } \\
(\%)\end{array}$ & $\begin{array}{l}\text { length } \\
\text { occupied } \\
\text { (bb) }\end{array}$ & $\begin{array}{l}\text { percentage } \\
\text { of sequence } \\
(\%)\end{array}$ \\
\hline 2009-605 & 0 & 0 & 0 & 0 & 1403090 & 4.12 \\
\hline 463 & 0 & 0 & 43660 & 0.13 & 1601065 & 4.7 \\
\hline $85 S$ & 2289.00 & 0.01 & 57668.00 & 0.16 & 3082210.00 & 8.58 \\
\hline CBS38166 & 0.00 & 0.00 & 29731.00 & 0.09 & 1625629.00 & 4.78 \\
\hline DVD161 & 1994.00 & 0.01 & 0.00 & 0.00 & 1871766.00 & 5.59 \\
\hline DVD31 & 15326.00 & 0.05 & 14424.00 & 0.04 & 1718276.00 & 5.12 \\
\hline DVD3 & 1923.00 & 0.01 & 17323.00 & 0.05 & 2262138.00 & 6.57 \\
\hline DVD-S26 & 0.00 & 0.00 & 34185.00 & 0.10 & 1847224.00 & 5.32 \\
\hline DVD-S29 & 22393.00 & 0.01 & 7832.00 & 0.02 & 1297199.00 & 3.91 \\
\hline DVD-S94 & 0.00 & 0.00 & 23539.00 & 0.07 & 2082451.00 & 6.05 \\
\hline JKG8 & 2294.00 & 0.01 & 0.00 & 0.00 & 1344573.00 & 3.97 \\
\hline ST100 & 1720.00 & 0.01 & 50597.00 & 0.14 & 2693973.00 & 7.71 \\
\hline ST14.01 & 0.00 & 0.00 & 30264.00 & 0.09 & 1610719.00 & 4.67 \\
\hline ST16.01 & 1582.00 & 0.00 & 50167.00 & 0.15 & 1009974.00 & 2.95 \\
\hline V152 & 0.00 & 0.00 & 40614.00 & 0.12 & 1660694.00 & 4.89 \\
\hline V52 & 3965.00 & 0.01 & 28553.00 & 0.09 & 1442720.00 & 4.30 \\
\hline VanDijk & 1954.00 & 0.01 & 0 & 0 & 1337583.00 & 4.03 \\
\hline Vd39 & 1582.00 & 0.00 & 71392.00 & 0.20 & 3201873.00 & 8.92 \\
\hline CQ2 & 3965.00 & 0.01 & 54386.00 & 0.15 & 3156817.00 & 8.81 \\
\hline$J R 2$ & 1954.00 & 0.01 & 98739.00 & 0.27 & 2841008.00 & 7.86 \\
\hline VdLs17 & 0.00 & 0.00 & 235025.00 & 0.65 & 2875975.00 & 7.99 \\
\hline
\end{tabular}




\section{Chapter 4}

$$
\text { do }
$$

$$
26
$$

co

ᄂ 0

$d \sigma$ 


\section{The Verticillium dahliae effector Sun1 quantitatively contributes to virulence on sunflower}

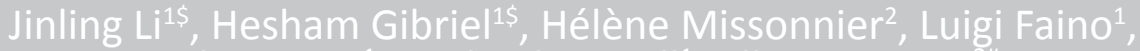
Xiaoqian Shi-Kunne ${ }^{1}$, Michael F. Seidl ${ }^{1}$, Alban Jacques ${ }^{2 \#}$, and Bart P.H.J. Thomma ${ }^{1 \#}$

1 Laboratory of Phytopathology, Wageningen University, Droevendaalsesteeg 1, 6708 PB Wageningen, The Netherlands.

2 Department of Physiologie, Pathologie et Génétique Végétales (PPGV), Université de Toulouse, INP-PURPAN, Toulouse, France, Syngenta France S.A.S., Saint-Sauveur, France.

\$ These authors contributed equally to this work

\# These authors contributed equally to this work

To be submitted for publication 


\begin{abstract}
Plant pathogens employ effector molecules to manipulate host physiology and establish themselves within their hosts. Verticillium dahliae is a highly destructive xylem-colonizing fungal pathogen that causes vascular wilt disease on diverse crops, such as tomato (Solanum lycopersicum), cotton (Gossypium hirsutum), and sunflower (Helianthus annuus). In this study, we show that only particular $V$. dahliae strains cause vascular wilt disease on sunflower. Based on comparative genomics of a sunflower pathogenic strain with a diversity of non-pathogenic strains, we identified two candidate effector genes that occur in the sunflower-pathogenic strain $85 \mathrm{~S}$ and that are highly expressed during host colonization. Intriguingly, these two candidate effector genes appeared to be identical copies that arose by a segmental duplication. Here, we show that this duplicated effector gene quantitatively contributes to $V$. dahliae virulence on sunflower but not on Nicotiana benthamiana or Arabidopsis thaliana.
\end{abstract}




\section{INTRODUCTION}

Plants and microbes engage in a diverse array of symbiotic relationships, ranging from pathogenic to mutualistic. Pathogenic interactions between plants and microbial pathogens have been described as ongoing arms races in which plants try to halt microbial ingress while pathogens attempt to continue symbiosis (Jones and Dangl 2006; Thomma et al. 2011; Cook et al. 2015). In these arms races, plants have developed various types of immune receptors to detect invading pathogens through sensing various pathogen-derived or pathogen-induced molecular patterns, so-called invasion patterns, that betray microbial invasion to activate appropriate immune responses (Cook et al., 2015; Rodriguez-Moreno et al., 2017). In turn, to enable a parasitic life on their hosts, microbial pathogens secrete so-called effector molecules to deregulate host immune responses and support successful host infection (Jones and Dangl, 2006; Rovenich et al., 2014; Cook et al., 2015). Whereas most extensively studied effectors are proteinaceous molecules, other types of microbially secreted molecules, such as secondary metabolites and small RNAs, have also been described as non-canonical effectors (Wang et al., 2015; Rodriguez-Moreno et al., 2018). Interestingly, ongoing research has revealed that effectors are not exclusively secreted by plant pathogens but other types of symbionts such as endophytes and mutualists similarly employ effectors to establish host interactions (Rovenich et al., 2014). For instance, the mutualistic fungus Laccaria bicolor secretes the effector protein MiSSP7 to interact with jasmonic acid (JA) signalling components of the host and facilitate symbiosis by suppressing host immunity (Plett et al., 2014). Moreover, as all symbionts establish themselves in environments that comprise other microbes including antagonists, effector molecules may also act in self-defense and competition with microbiome co-inhabitants (Rovenich et al., 2014; Snelders et al., 2018).

Vascular wilts caused by soil-borne fungal species of the Verticillium genus are among the most devastating plant diseases worldwide with an estimated annual loss of $€ 3$ billion in the 20 most affected hosts (Depotter et al., 2016). Within the Verticillium genus, $V$. dahliae is the most notorious pathogenic species that has the ability to infect over 200 plant species including high-value crops such as tomato (Solanum lycopersicum), cotton (Gossypium hirsutum), and sunflower (Helianthus annuus) (Inderbitzin and Subbarao, 2014). V. dahliae invades plant hosts via roots and then colonizes the water-conducting xylem vessels, which disrupts water transport and causes the characteristic wilting symptoms (Fradin and Thomma, 2006; Klosterman et al., 2011). Verticillium wilt disease management is notoriously challenging since conventional fungicides are generally ineffective to eradicate the pathogen once it has entered the xylem tissues (Fradin and Thomma, 2006; Klosterman et al., 2009). Moreover, due to the broad host range of the pathogen and long viability of the resting structures in the soil, cultivation practices such as crop rotation do not result in efficient disease management (Pegg and Brad, 2002; Fradin and Thomma, 2006). Although genetic resistance has been considered as a preferred strategy for disease control, only few Verticillium wilt resistance genes have 
been identified so far (Schaible et al., 1951; Simko et al., 2004; Fradin and Thomma, 2006). For example, in tomato, immune receptor Ve1 confers resistance to race $1 \mathrm{~V}$. dahliae and Verticillium albo-atrum strains through recognition of the race 1-specific effector protein Ave1 (Fradin et al., 2009; de Jonge et al., 2012). Transfer of tomato Ve1 into other crop species like tobacco and cotton can provide resistance against race 1 Verticillium strains (Song et al., 2018). The high economic impact of Verticillium wilt disease, combined with the absence of curative treatments, substantiates the need for developing novel disease control strategies. Recently, host-induced gene silencing (HIGS), which involves host expression of double-stranded RNAs to target and silence essential pathogen genes to confer host resistance, has been developed for controlling Verticillium wilt disease in cotton and tomato (Song and Thomma, 2016; Zhang et al., 2016).

To design novel control strategies to combat Verticillium wilt disease, a thorough understanding of molecular mechanisms underlying pathogenesis of $V$. dahliae is of fundamental importance (Fradin and Thomma, 2006). Over the past years, forward genetic approaches such as random mutagenesis and proteomic techniques have been performed to identify potential pathogenicity and virulence factors of $V$. dahliae (ElBebany et al., 2010; Santhanam, 2014; Chen et al., 2016; Zhang et al., 2017). The ease and low cost of present-day genome sequencing (Faino and Thomma, 2014; Gibriel et al., 2016) have made it possible to sequence multiple $V$. dahliae isolates from various host niches. Using comparative population genomics, we previously identified the $V$. dahliae race-specific effector Ave1 that is crucial for fungal aggressiveness during tomato colonization (de Jonge et al., 2012). Subsequently, Ave1 was demonstrated to contribute to fungal virulence not only on tomato plants that lack the Ve1 resistance gene, but also on tobacco, Arabidopsis, as well as on cotton (de Jonge et al., 2012; Song et al., 2018). More recently, we applied comparative genomics to identify the $V$. dahliae defoliating (D) pathotype-specific effector (named D effector) that is responsible for defoliation symptoms on cotton and olive (Li et al. unpublished). Interestingly, we found that the $D$ effector acts as a pathogenicity determinant of $V$. dahliae on diverse host species (Li et al. unpublished). Using a similar approach, we uncovered a $V$. dahliae effector (named Tom 1 ) that mediates pathogenicity of $V$. dahliae on tomato (Li et al. unpublished).

Sunflower is a worldwide planted oil crop that is known to maintain stable yields across a wide variety of environmental conditions (Kane and Rieseberg, 2007). However, Verticillium wilt disease constitutes an important constraint for the production of sunflower (Yao et al., 2011; Guo et al., 2017). Despite huge yield losses caused by $V$. dahliae, so far relatively little is known about how the pathogen causes disease on sunflower and no effectors have been characterized for facilitating fungal aggressiveness on sunflower. In this study, comparative genome analysis of a sunflower pathogenic strain with multiple non-pathogenic strains was performed to identify $V$. dahliae effector gene candidates that are essential for virulence on sunflower. 


\section{MATERIALS AND METHODS}

\section{Fungal strains and plant materials}

Verticillium dahliae strains (Table S1) were grown on potato dextrose agar (PDA) at $22^{\circ} \mathrm{C}$. Sunflower ( $H$. annuus $\mathrm{cv}$. Tutti), Nicotiana benthamiana, cotton ( $G$. hirsutum $\mathrm{cv}$. Simian3), tomato (S. lycopersicum cv. Moneymaker), and Arabidopsis thaliana (Col-0) plants were grown under controlled greenhouse conditions (Unifarm, Wageningen, The Netherlands) at $21^{\circ} \mathrm{C} / 19^{\circ} \mathrm{C}$ during $16 / 8$ hours day/night periods, respectively, with $70 \%$ relative humidity and $100 \mathrm{~W} / \mathrm{m}^{2}$ supplemental light when the light intensity dropped below $150 \mathrm{~W} / \mathrm{m}^{2}$.

\section{V. dahliae genome sequences and phylogenetic tree construction}

Genomes of the four $V$. dahliae strains (JR2, VdLs17, CQ2, and 85S) were sequenced using Single-Molecule Real-Time (SMRT) sequencing technology (Faino et al., 2015; Depotter et al., 2018) (Table S1). Additionally, genomes of V. dahliae strains V574 and BP2 were sequenced using the Illumina HiSeq 2000 (Li et al. unpublished) (Table S1). A phylogenetic tree of the $V$. dahliae strains was generated with REALPHY (version 1.12) (Bertels et al., 2014) using Bowtie2 (Langmead and Salzberg, 2012) to map genomic reads against the reference $V$. dahliae strain JR2. A maximum likelihood phylogenetic tree was inferred using RAxML (version 8.2.8) with the GTRGAMMA model and 500 bootstrap replicates (Stamatakis, 2014).

\section{V. dahliae strain $85 \mathrm{~S}$ effector identification}

In order to identify effector genes of $V$. dahliae strain $85 \mathrm{~S}$ that mediate aggressiveness on sunflower plants, we mapped DNA reads from non-pathogenic strains to the genome assembly of $V$. dahliae strain $85 \mathrm{~S}$ using BWA (BWA-mem algorithm) (v0.7.12) (Li and Durbin, 2010). Read coverage mapping of all $V$. dahliae strains over the $85 \mathrm{~S}$ reference genome was calculated in $100 \mathrm{bp}$ windows using BEDTools coverage (v2.25) (Quinlan and Hall, 2010). Genomic regions were considered present if the breadth of coverage $\geq 50 \%$, while those with breadth of coverage $<50 \%$ were considered absent. lineagespecific (LS) genomic regions, here defined as genomic regions that are only present in $V$. dahliae strain $85 \mathrm{~S}$ and absent in all other non-pathogenic $V$. dahliae strains, were determined and genes localized within these LS regions were extracted using BEDtools intersect (v2.25) (Quinlan and Hall, 2010).

A gene annotation for $V$. dahliae strain $85 \mathrm{~S}$ was generated using the Maker2 pipeline (Holt and Yandell, 2011). To identify potential effector genes of strain 85S, N-terminal signal peptides were first predicted with SignalP (version 4.1) (Petersen et al., 2011). Subsequently, the machine-learning approach applied in EffectorP (version 1.0) (default parameters) was used (Sperschneider et al., 2016). Effector genes localized within LS regions were extracted using BEDtools intersect (Quinlan and Hall, 2010). Syntenies between effector gene copies was analysed using NUCmer (version 3.1) (--maxmatch), 
which is part of the MUMer package (Kurtz et al., 2004), and visualized using R package genoPlotR (v0.8.6) (Guy et al., 2010).

\section{Gene expression analysis}

To determine expression of Sun1 in planta, 12-day-old sunflower seedlings were rootinoculated with conidiospores of $V$. dahliae strain $85 \mathrm{~S}$ as described previously (Fradin et al., 2009). Stems of inoculated sunflower plants were harvested at 9, 16, 24, 32 and 40 days post inoculation (dpi), flash frozen in liquid nitrogen and stored at $-80^{\circ} \mathrm{C}$ for total RNA extraction. Total RNA extraction and cDNA synthesis were performed as described previously (Song et al., 2016). Quantitative real time PCR was preformed to detect the expression of Sun1 using primers Sun1-F(RT-PCR) and Sun1-R(RT-PCR), whereas the $V$. dahliae GAPDH (glyceraldehyde-3-phosphate dehydrogenase) gene served as an endogenous control (Table S2).

\section{Generation of Sun1 deletion strains}

To generate single Sun1 gene deletion constructs, sequence stretches of approximately $1.1 \mathrm{~kb}$ upstream and $1.2 \mathrm{~kb}$ downstream of the Sun 1 coding sequence were amplified from genomic DNA of $V$. dahliae strain 855 using primer pairs SKO-Sun1-LBF/LBR and SKO-Sun1-RBF/RBR (Table S2). The amplicons were cloned into the pRF-HU2 vector as described previously (Frandsen et al., 2008) and the resulting deletion construct was transformed into strain $85 \mathrm{~S}$ via Agrobacterium tumefaciens-mediated transformation as described earlier (Santhanam, 2012). Putative deletion transformants were selected on PDA supplemented with cefotaxime $(200 \mu \mathrm{g} / \mathrm{mL})$ and hygromycin $(50 \mu \mathrm{g} / \mathrm{mL})$ and homologous gene replacement was verified by PCR analysis using outsider primer $\mathrm{F}$ and outsider primer R (Table S2).

To generate Sun 1 double deletion mutants, sequence stretches of approximately $1.3 \mathrm{~kb}$ upstream and $1.1 \mathrm{~kb}$ downstream of the Sun1 coding sequence were amplified using primer pairs DKO-Sun1-LBF/LBR and DKO-Sun1-RBF/RBR (Table S2). The amplified products were cloned into vector pRF-NU2. Next, the gene replacement construct was transformed into a Sun1 single deletion mutant. Putative double deletion transformants were selected on PDA supplemented with nourseothricin $(15 \mu \mathrm{g} / \mathrm{mL})$ and hygromycin $(50 \mu \mathrm{g} / \mathrm{mL})$ and confirmed by PCR analysis using outsider primer $\mathrm{F}$ and outsider primer $\mathrm{R}$ (Table S2).

\section{Pathogen inoculations and fungal recovery assays}

Inoculations were performed on sunflower, cotton, tomato, $N$. benthamiana, and A. thaliana plants as previously described (Fradin et al., 2009). Disease symptoms were scored up to 21 (tomato, A. thaliana, and N. benthamiana), 28 (cotton), or 45 (sunflower) days post inoculation (dpi). Plants were photographed and Image J was used to determine the canopy area while a rectilinear scale was used to measure plant height. Fungal biomass in $A$. thaliana, $N$. benthamiana, and sunflower were determined as previously described (Song et al., 2018). Stems of five inoculated plants were 
harvested at $21 \mathrm{dpi}$ ( $A$. thaliana and N. benthamiana), or $45 \mathrm{dpi}$ (sunflower) for genomic DNA extraction. Real-time quantitative PCR ( $q P C R$ ) was conducted to quantify fungal colonization (Ellendorff et al., 2009) using the fungus-specific primers ITS-F and ITS-R (Table S2). Primers targeting the sunflower elongation factor 1-alpha (EF-1 $\alpha)$ gene, $A$. thaliana RuBisCo, and $N$. benthamiana RuBisCo gene were used as endogenous plant controls as described earlier (Table S2) (Song et al., 2018).

Fungal recovery assays were conducted to detect $V$. dahliae strains in planta as previously described (Fradin et al., 2009). Stem sections were harvested at 45 days post inoculation (dpi), surface-sterilized and sliced into small discs. For each $V$. dahliae strain, 12 to 15 stem slices from five pooled plants transferred onto PDA supplemented with chloramphenicol $(35 \mu \mathrm{g} / \mathrm{mL})$ and incubated at $22^{\circ} \mathrm{C}$ for 7 days.

\section{RESULTS}

\section{Verticillium dahliae inoculations on sunflower}

Various strains of $V$. dahliae have been sequenced in our laboratory but their capacity to infect sunflower remained unknown (Klosterman et al., 2011; de Jonge et al., 2013; Thomma unpublished data). Therefore, in addition to the sunflower-pathogenic $V$. dahliae strain 85S, five strains of which the genome has been sequenced were tested for their ability to infect sunflower. These comprise JR2 and VdLs17, for which a gapless genome assembly has previously been generated (Faino et al., 2015) and that are known to cause Verticillium wilt on tomato and lettuce, respectively, and three $V$. dahliae strains that are phylogenetically distant from JR2 and VdLs17, namely the cotton-pathogenic strains CQ2, BP2 and V574 (Figure S1). Interestingly, in contrast to strain 85S that induced clear stunting, chlorosis and wilting on inoculated sunflower plants, JR2, CQ2, VdLs17, BP2 and V574 failed to cause visible disease symptoms, suggesting that these strains do not have the capacity to infect sunflower (Figure $1 A-B$ ). This finding was further corroborated by fungal recovery assays, as plating of stem sections of the inoculated plants on agar medium resulted in fungal outgrowth from all sections of 85S-inoculated plants while no fungal growth was recovered from stem sections of plants inoculated with any of the other strains (Figure $1 C$ ). Thus, except for $V$. dahliae strain 855 that can be classified as a sunflower pathogenic strain, all other strains (JR2, CQ2, VdLs17, BP2 and V574) were classified as non-pathogenic on sunflower. 
A
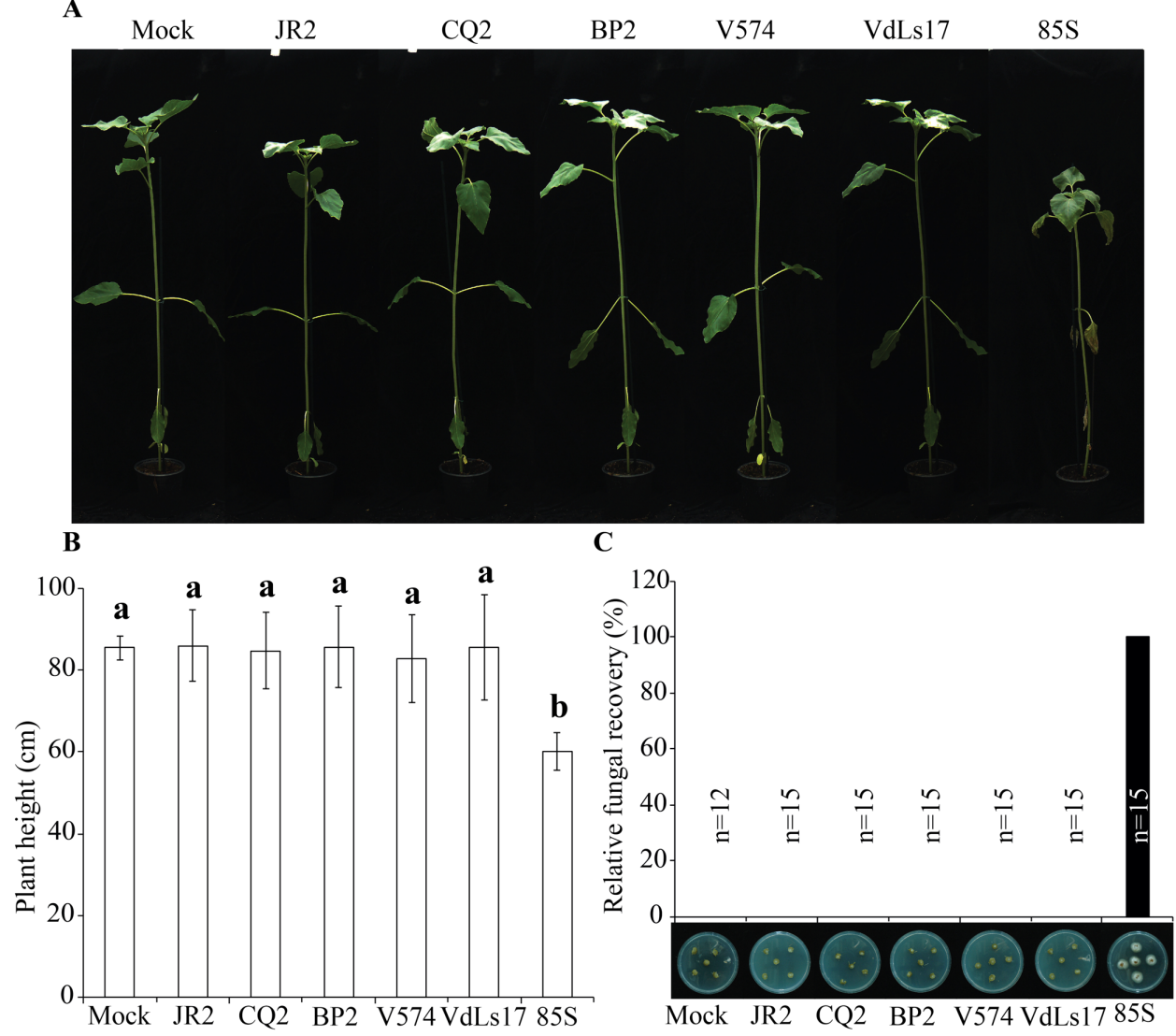

Figure 1. Phenotypes of sunflower plants inoculated with sequenced Verticillium dahliae strains.

(A) Side view of sunflower (cv. Tutti) plants at 45 days after mock-inoculation or inoculation with V. dahliae strains JR2, CQ2, BP2, V574, VdLs17 and 85S.Besides the stunting, the plant inoculated with strain $85 \mathrm{~S}$ also displays chlorosis and wilting symptoms. (B) Quantification of $V$. dahliae-induced plant stunting at 45 days post inoculation (dpi). Bars represent the average height of five plants with standard deviation. Different letter labels indicate statistically significant differences (Student's $t$-test; $P<0.05$ ). (C) Fungal outgrowth at 10 days after plating of sunflower stem sections harvested at 45 days post inoculation (dpi) with V. dahliae strains JR2, CQ2, BP2, V574, VdLs17 and 85S. The bar graph shows the percentage of stem section slices from which fungal outgrowth was observed. Inoculation experiments were performed with five plants for each fungal strain and independently repeated twice with similar results. 


\section{Comparative genomics identifies the effector gene Sun1}

We aimed to identify effectors that mediate aggressiveness on sunflower plants by comparative genomics of pathogenic strain $85 \mathrm{~S}$ and the non-pathogenic strains JR2, VdLs17, CQ2, BP2, and V574. To this end, the genomes of $V$. dahliae strains 85S, CQ2, VdLs17, as well as JR2 were sequenced with PacBio technology (Faino et al., 2015; Depotter et al., 2018). In addition, the genomes of BP2 and V574 were sequenced by using the Illumina HiSeq 2000 (Li et al. unpublished).

Gene annotation for $V$. dahliae strain $85 \mathrm{~S}$ using the Maker2 pipeline (Holt and Yandell, 2011) yielded a total of 10,580 protein-coding genes. Of these genes, 1,062 genes were predicted to encode putative secreted proteins. Subsequently, we used the machinelearning approach applied in EffectorP (Sperschneider et al., 2016) to identify 174 candidate effectors for $V$. dahliae strain $85 \mathrm{~S}$. To identify $85 \mathrm{~S}$-specific effector genes, a reference-based alignment strategy was used. All reads of the non-pathogenic $V$. dahliae strains were mapped onto the genome assembly of strain $85 \mathrm{~S}$ and lineage specific (LS) regions for which no synteny occurred in any of the non-pathogenic strains were extracted. This comparative analysis revealed 6,924 LS sequences of $100 \mathrm{bp}$ in size, collectively comprising 159 genes including five that encode secreted proteins. Of these five genes, two were classified as effector genes based on EffectorP. Remarkably, both effector genes were found to encode a putative effector of 125 amino acids and share $100 \%$ sequence identity, despite the observation that they are located on two different contigs, namely unitig_4 (292.60 kb) and unitig_14 (693.13 kb) (Figure 2A). Moreover, further alignment revealed that both contigs are highly syntenic ( $99 \%$ identity) (Figure $2 \mathrm{~B})$, suggesting that they arose from a recent segmental duplication. We named the putative effector genes Sun1- $a$ and Sun1-b, as candidates for mediating virulence on sunflower. Similar to most fungal effectors reported so far (Stergiopoulos and de Wit, 2009; de Jonge et al., 2011 ), no Sun1 homologs were found in other species. 


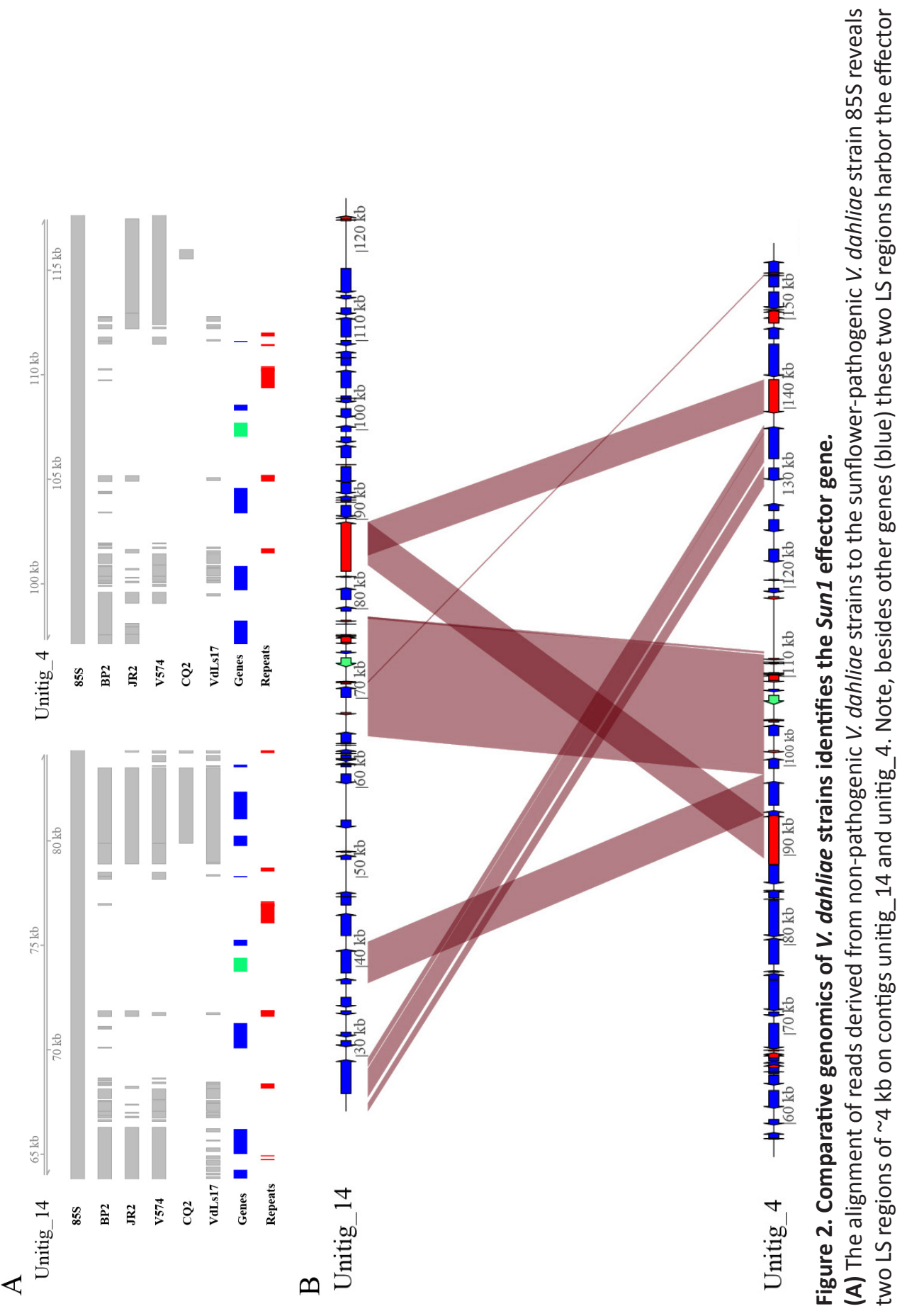


gene Sun1 (green) that is flanked by repeats (red), and solely present in the strain $85 \mathrm{~S}$. (B) Synteny analyses of unitig_4 and unitig_14 harboring the Sun1 effector gene. A region encompassing $50 \mathrm{~kb}$ upstream and downstream of Sun1 effector gene is shown. Highly syntenic regions (99\% identity) upstream and downstream of this effector gene on unitig_14 and unitig_4 are indicated with red ribbons.

To further assess the potential of Sun1 as a virulence effector on sunflower, the transcript level of Sun1 during infection of $V$. dahliae strain $85 \mathrm{~S}$ on sunflower was investigated using real-time PCR in a time course experiment up to 40 days post inoculation (dpi). This analysis revealed that Sun 1 transcript levels increased significantly at 9 days postinoculation (dpi) and remained high at later time points (Figure 3).

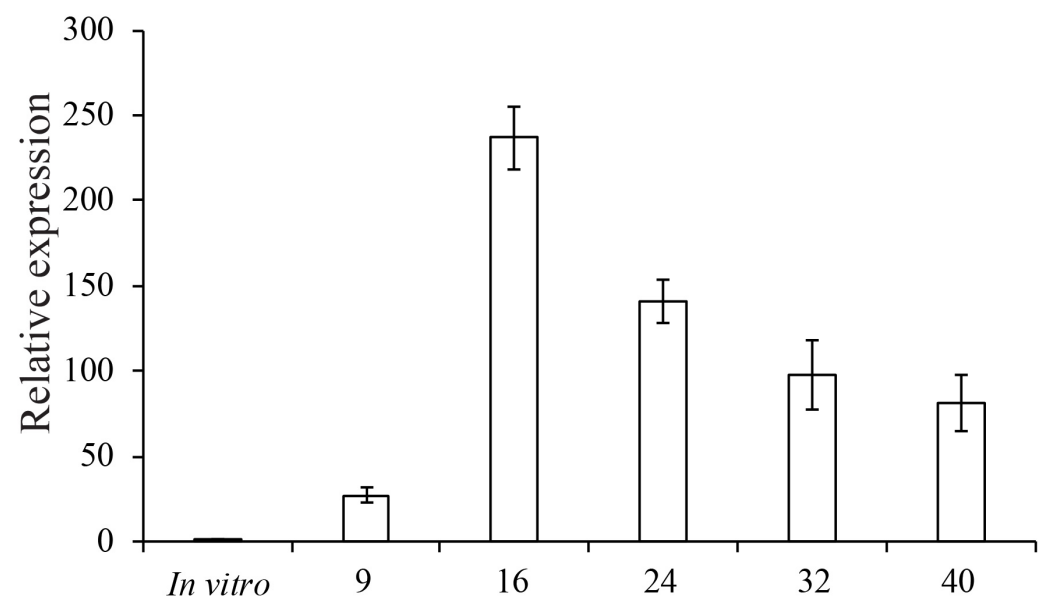

Figure 3. Sun1 is highly induced during infection of Verticillium dahliae on sunflower. Twelve day-old sunflower (cv. Tutti) seedlings were root-inoculated with $V$. dahliae strain $85 \mathrm{~S}$ and stems were harvested at 9, 16, 24, 32 and 40 days post inoculation (dpi). After RNA isolation and cDNA synthesis, real-time PCR was performed to determine the relative expression level of Sun 1 using the $V$. dahliae GAPDH gene as a reference. Expression of Sun 1 in vitro (potato dextrose agar) was set to 1. 
A

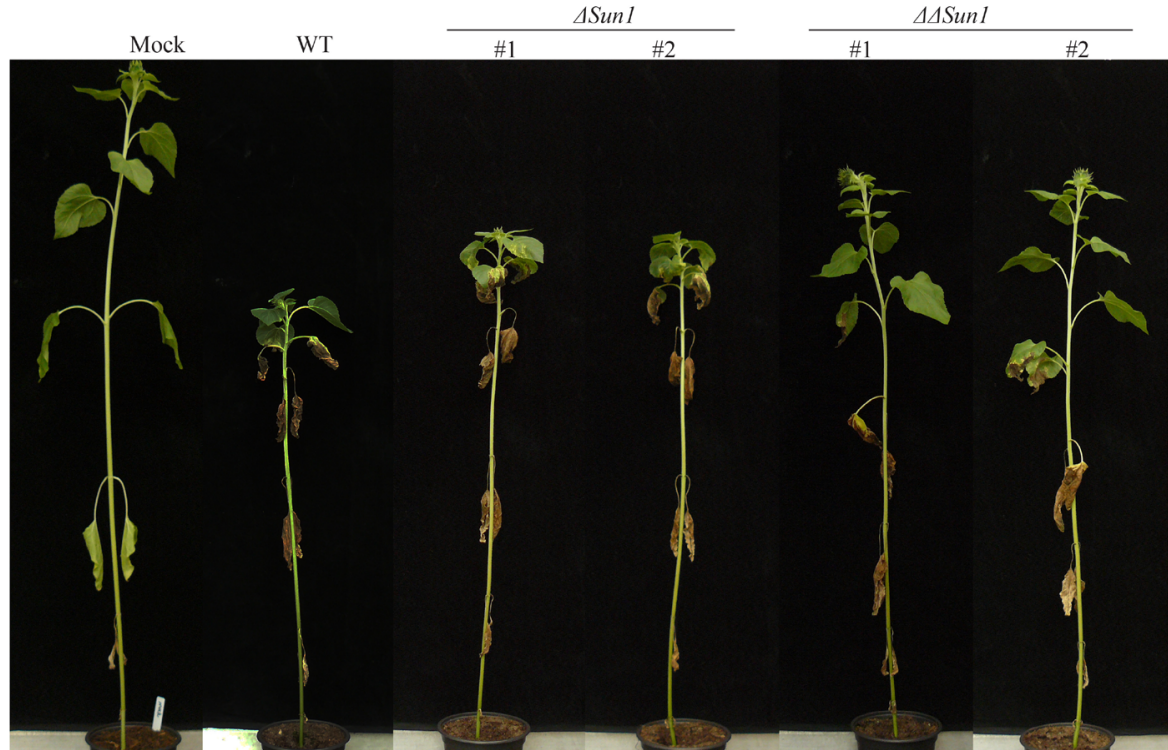

B

C
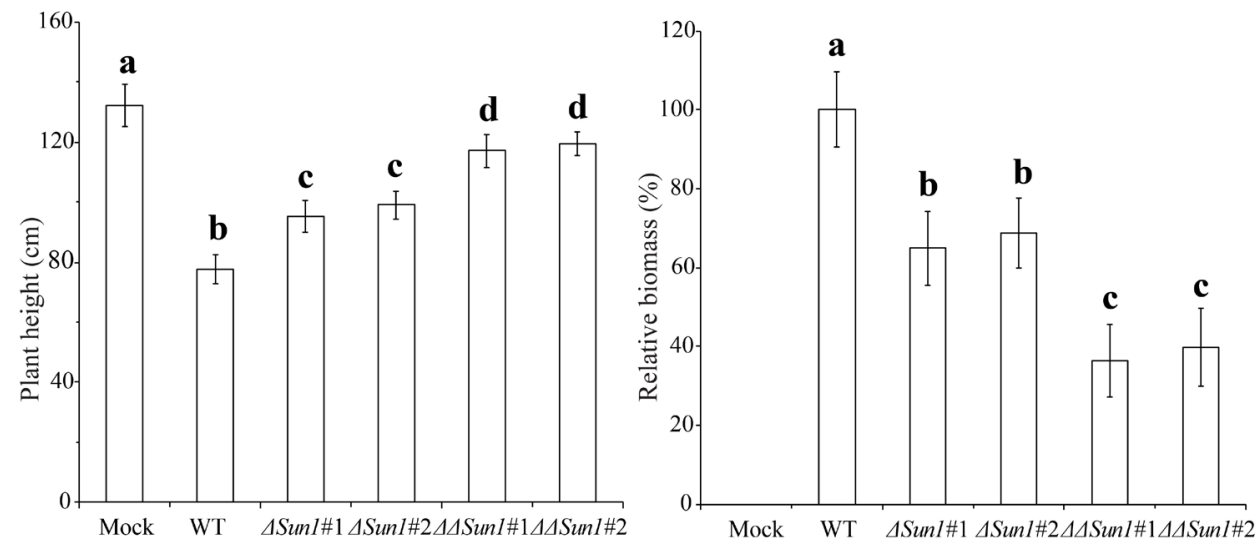

Figure 4. Sun1 is required for full virulence on sunflower.

(A) Typical phenotype of sunflower (cv. Tutti) upon mock-inoculation or inoculation with wild-type strain $85 \mathrm{~S}$ (WT), two Sun1 single deletion strains ( $\triangle$ Sun1\#1 and $\triangle$ Sun1\#2) and two Sun1 double deletion strains ( $\triangle \Delta S$ Sun1\#1 and $\Delta \Delta$ Sun1\#2). Photographs were taken at 45 days post inoculation (dpi). (B) Quantification of $V$. dahliae-induced plant stunting at $45 \mathrm{dpi}$. Bars represent the average height of five plants with standard deviation. (C) Fungal biomass as determined with real-time PCR at $45 \mathrm{dpi}$. Bars represent $V$. dahliae ITS levels relative to sunflower elongation factor 1 - $\alpha$ levels (for equilibration) with standard deviation in a sample of five pooled plants. The fungal biomass in sunflower plants upon inoculation with 
the wild-type strain $85 \mathrm{~S}$ is set to $100 \%$. Different letter labels indicate statistically significant differences (Student's $t$-test; $P<0.05$ ). Inoculation experiments were performed with five plants for each fungal strain and independently repeated twice with similar results.

\section{The Sun1 effector quantitatively contributes to virulence on sunflower}

To further assess the contribution of the Sun 1 effector to virulence of $V$. dahliae strain $85 \mathrm{~S}$ on sunflower, we generated deletion mutants for both effector gene copies through homologous recombination (Figure S2A). To this end, we first generated single gene copy deletion mutants, followed by deletion of the second Sun 1 gene copy. Putative single and double gene deletion mutants were confirmed by PCR (Figure S2B). Thereby, we also confirmed that the predicted presence of the two gene copies is genuine and not the consequence of an assembly artefact (Figure S2B). Two independent single ( $\triangle$ Sun1\#1 and $\Delta$ Sun1\#2) and two independent double ( $\Delta \Delta$ Sun1\#1 and $\Delta \Delta S$ Sun1\#2) gene deletion mutants were selected for subsequent inoculation assays.

Single deletion ( $\triangle$ Sun 1 ) mutants exhibited markedly reduced virulence on sunflower when compared with wild-type strain $85 \mathrm{~S}$ at 45 days post inoculation (dpi) (Figure 4A), demonstrated by significantly reduced stunting and compromised fungal colonization of the host plants (Figure 4B-C). Interestingly, sunflower plants that were inoculated with double deletion ( $\triangle \Delta$ Sun 1 ) mutants showed a similar disease phenotype as plants upon inoculation with $\Delta S$ Sun 1 mutants during relatively early stages of infection up until $24 \mathrm{dpi}$, as no significant difference in disease symptoms and plant height reduction was observed. However, from 32 dpi onwards, sunflower plants that were inoculated with $\Delta \Delta$ Sun1 mutants showed significantly less wilting symptoms and stunting than plants inoculated with $\triangle S$ Sun1 mutants (Figure 4B). Moreover, real-time PCR quantification of fungal biomass demonstrated that $\Delta \Delta S$ Sn 1 mutants accumulated significantly less fungal biomass during infection than $\Delta S u n 1$ mutants and the wild type strain (Figure 4C). Taken together, these data demonstrate that the Sun1 effector quantitatively contributes to virulence on sunflower plants.

\section{The Sun1 effector is dispensable for virulence on Nicotiana benthamiana and Arabidopsis thaliana}

To investigate whether the observed role of Sun1 in virulence is confined to sunflower only or also extends to other host species, we first tested the virulence of the $V$. dahliae strain $85 \mathrm{~S}$ on the Solanaceous crop tomato, the model plant $N$. benthamiana, the Malvaceae crop cotton and the Brassicaceous model plant $A$. thaliana. While 85S failed to cause wilt disease on tomato (Figure S3) and caused only mild wilt disease symptom on cotton (Figure S4), clear symptoms of disease were observed on $A$. thaliana and $N$. benthamiana upon inoculation. Next, we examined the virulence of Sun1 deletion mutants ( $\Delta$ Sun 1 and $\Delta \Delta$ Sun 1 ) on $A$. thaliana and $N$. benthamiana to evaluate the contribution of this effector to virulence on these two hosts. However, unlike our observations on sunflower, deletion of Sun1 did not result in significantly compromised virulence on $N$. benthamiana, as similar Verticillium wilt symptoms, including wilting 
and stunting, were observed for $\Delta S u n 1$ and $\Delta \Delta S u n 1$ mutants as for the wild-type strain $85 \mathrm{~S}$ at 3 weeks post inoculation (Figure 5A, C). Real time PCR quantification of fungal biomass confirmed that all strains colonized $N$. benthamiana plants to a similar extent (Figure 5D). Similarly, Sun1 effector was also not found to contribute to virulence on $A$. thaliana (Figure 5B, E-F). Therefore, we conclude that the Sun1 effector does not play a general role in virulence, but its contribution appears to be confined to virulence on sunflower.

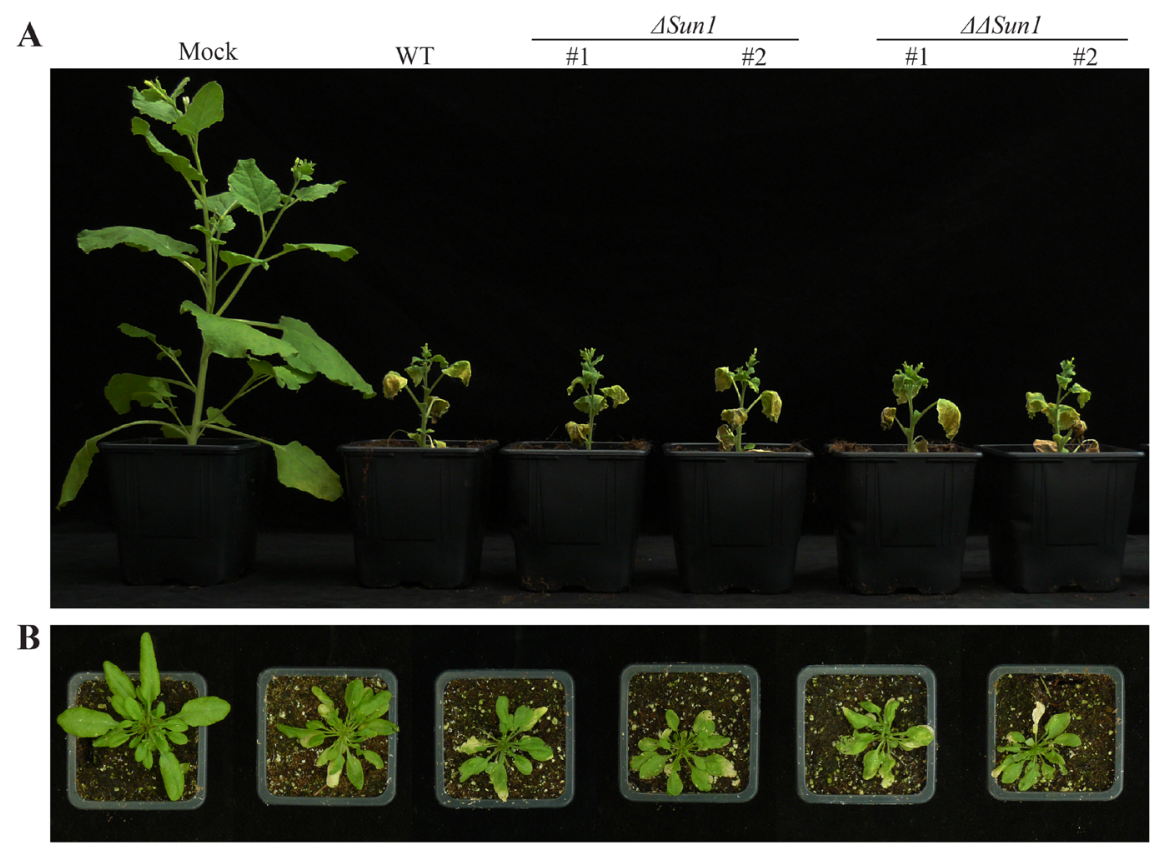

Figure 5. Sun1 is dispensable for virulence on Nicotiana benthamiana and Arabidopsis thaliana.

(A) Typical phenotype of $N$. benthamiana plants that were mock-inoculated or inoculated with wild type strain $85 S$ (WT), two Sun1 single deletion strains ( $\triangle$ Sun1\#1 and $\triangle$ Sun1\#2) and two Sun1 double deletion strains ( $\Delta \Delta S$ Sun1\#1 and $\Delta \Delta S$ Sun1\#2). (B) Typical phenotype of A. thaliana (Col-0) plants that were mock-inoculated or inoculated with indicated fungal strains in panel A at 21 (dpi). 
C

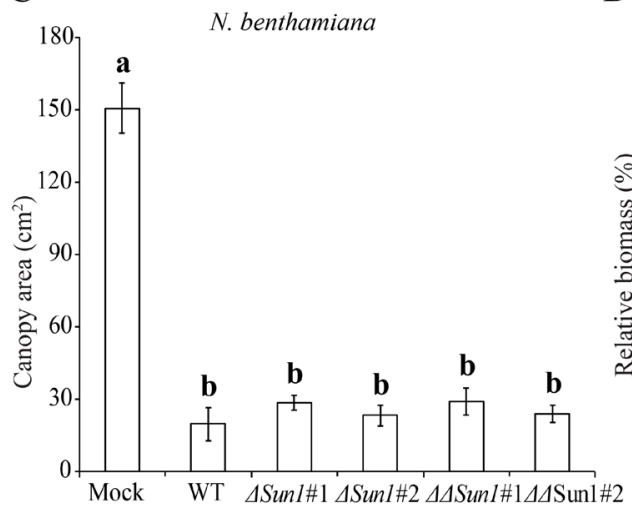

D

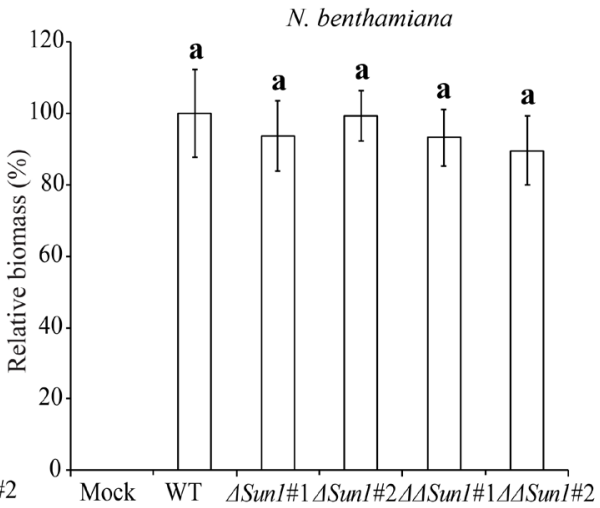

E

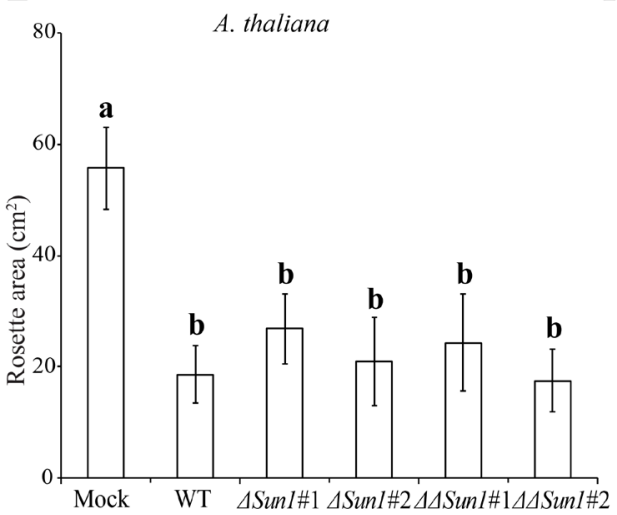

F

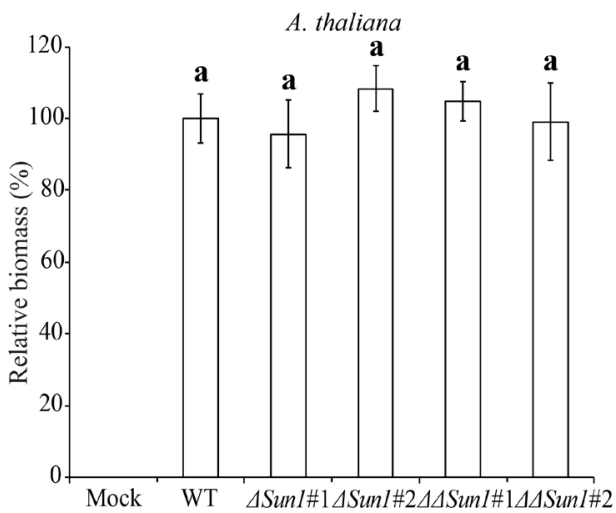

Figure 5 continued. Sun1 is dispensable for virulence on Nicotiana benthamiana and Arabidopsis thaliana. (C) Quantification of the canopy area of $N$. benthamiana at $21 \mathrm{dpi}$. Bars represent the average canopy area of five plants with standard deviation. Different letter labels indicate statistically significant differences (Student's $t$-test; $P<0.05$ ). (D) Fungal biomass as determined with real-time PCR at $21 \mathrm{dpi}$. Bars represent $V$. dahliae ITS levels relative to $N$. benthamiana RuBisCo levels (for equilibration) with standard deviation in a sample of five pooled plants. The fungal biomass in $N$. benthamiana plants upon inoculation with the wild-type strain 85 is set to $100 \%$. The same letter labels indicate no statistically significant differences (Student's $t$-test; $P<0.05$ ). (E) Quantification of the rosette area of five $A$. thaliana plants at $21 \mathrm{dpi}$. Bars represent the average rosette area of five plants with standard deviation. Different letter labels indicate statistically significant differences (Student's $t$-test; $P<0.05$ ). (F) Fungal biomass as determined with real-time PCR at $21 \mathrm{dpi}$. Bars represent $V$. dahliae ITS levels relative to $A$. thaliana RuBisCo levels (for equilibration) with standard deviation in a sample of five pooled plants. The fungal biomass in $A$. thaliana plants upon inoculation with the wild-type strain $85 \mathrm{~S}$ is set to $100 \%$. The same letter labels 
indicate no statistically significant differences (Student's $t$-test; $P<0.05)$. Photographs were taken at 21 days post inoculation (dpi). Inoculation experiments were performed with five plants for each fungal strain and independently repeated twice with similar results.

\section{DISCUSSION}

While $V$. dahliae strains are typically characterized by their broad host range, pathogenic isolates still display differential host specificity as individual isolates only infect a limited number of plant species (Bhat and Subbarao, 1999). Over the years, a number of studies have suggested that the host range of a given pathogen is largely governed by the presence of host-specific virulence genes that contribute to disease establishment (van der Does and Rep, 2007). Like many filamentous pathogens, $V$. dahliae also employs effector molecules as virulence factors to establish disease (Klimes et al., 2015). To date, a number of $V$. dahliae effectors have been reported to contribute to disease establishment on various hosts. For example, the effector proteins Ave1 and NLP1 (necrosis-and ethylene-inducing-like protein 1) have been shown to function as virulence factors on multiple host plants, such as tomato, $N$. benthamiana, as well as A. thaliana (de Jonge et al., 2012; Song et al., 2018; Santhanam et al., 2013). The recently identified $D$ effector was shown to act as a pathogenicity factor of $V$. dahliae defoliating (D) pathotype strains on cotton, olive, N. benthamiana and $A$. thaliana (Li et al. unpublished). In contrast, other $V$. dahliae effectors may promote fungal virulence only on particular host species or genotypes. For instance, the chitin-binding lysin motif (LysM) effector Vd2LyM was reported to contribute to fungal virulence on tomato, but not on N. benthamiana or A. thaliana (Kombrink et al., 2017). Similarly, the effector NLP2 is required for full virulence of $V$. dahliae strain JR2 on tomato and $A$. thaliana, but not on $N$. benthamiana (Santhanam et al., 2013). In addition, the Tom1 effector was found to function as a pathogenicity effector that determines host specificity of $V$. dahliae on tomato only (Li et al. unpublished). Similarly, in this study we observed that the Sun1 effector quantitatively contributes to fungal aggressiveness on sunflower (Figure 4), but not on N. benthamiana or A. thaliana (Figure 5). Thus, we anticipate that the Sun1 effector may specifically facilitate fungal virulence on sunflower, but future experiments on other hosts are needed to confirm this hypothesis.

Various mechanisms have been described that can facilitate the development of effector gene repertoires in pathogenic microbes, such as genome hybridization (Stukenbrock et al., 2012), gene duplication (Dutheil et al., 2016), and horizontal gene transfer (HGT) (Friesen et al., 2006; de Jonge et al., 2012). It has been suggested that the wellcharacterized $V$. dahliae LS effector Ave1 has been acquired from plants via HGT (de Jonge et al., 2012). Genomic comparisons of multiple $V$. dahliae strains revealed that all $V$. dahliae strains carry highly variable LS genomic regions, accounting for $1-5 \mathrm{Mb}$ of their $35-\mathrm{Mb}$ genome, that are unique or shared by only a sub-set of $V$. dahliae isolates (de Jonge et al., 2013; Faino et al., 2015, 2016). Interestingly, numerous in-planta- 
induced effector genes reside in LS genomic regions that largely consist of segmental genomic duplications, suggesting that gene duplications may play an important role in the emergence of effector genes in V. dahliae (Jonge et al., 2013; Faino et al., 2016). In the present study, we show that two identical Sun1 effector genes arose by a segmental genomic duplication event and the high level of similarity between flanking sequences of the Sun1 effector gene suggests that this duplication occurred rather recently (Figure 2 ). In line with this finding, two exact copies of the $D$ effector gene in $V$. dahliae $D$ pathotype strains have emerged by a recent segmental duplication as well (Li et al. unpublished). The relevance of the occurrence of two identical effector gene copies and their impact on fungal adaption remains unknown at this point. Possibly, the emergence of two copies of the effector gene is relevant to maintain fungal aggressiveness on host plants. In this light it is interesting to note that the functionality of the two copies is not redundant, as they both quantitatively contribute to virulence and, consequently, deletion of a single copy markedly affects fungal virulence.

Recent duplications of effector genes can be subjected to subsequent evolutionary diversification, leading to novel or altered functionality of one of the two gene copies (Sanchez-Vallet et al., 2018). Thus, effector genes of filamentous pathogens that arise from gene duplication events typically evolve in a so called "duplication-divergence" pattern: following a gene duplication event, one gene copy diverges to some extent due to functional redundancy and evolves a distinct function (Plissonneau et al., 2017). For example, gene duplications followed by sequence divergence were proposed to be responsible for the generation of novel effector genes in the smut fungus Ustilago maydis (Dutheil et al., 2016). Similarly, a large number of effector genes of the oomycete pathogen Phytophthora sojae underwent sequence diversification after gene duplication (Shen et al., 2013). In addition to experiencing functional diversification, the recent duplications of effector genes may also be subject to differential loss of the duplicated gene copies (Dong et al., 2015; Pedersen et al., 2012). Frequent effector gene losses after segmental duplications have been proposed to occur in the powdery mildew fungus Blumeria graminis, which contribute to the diversity of the effector repertoires of the pathogen (Wicker et al., 2013; Menardo et al., 2017). Possibly, selective forces from host immune systems contribute to this process. However, it is unclear whether either of the two copies of the Sun 1 effector gene will experience sequence divergence or gene loss over time.

\section{ACKNOWLEDGEMENTS}

J.L. acknowledges receipt of a PhD fellowship from the China Scholarship Council (CSC). MFS acknowledges the receipt of a VENI grant of ALW-NWO. B.P.H.J.T. is supported by the Research Council for Earth and Life sciences (ALW) of the Netherlands Organization for Scientific Research (NWO). 


\section{REFERENCES}

Bhat RG, Subbarao KV. 1999. Host range specificity in Verticillium dahliae. Phytopathology 89: 1218-1225.

Chen JY, Xiao HL, Gui YJ, Zhang DD, Li L, Bao YM, Dai XF. 2016. Characterization of the Verticillium dahliae exoproteome involves in pathogenicity from cotton-containing medium. Frontiers in Microbiology 7: 1709.

Cook DE, Mesarich CH, Thomma BPHJ. 2015. Understanding plant immunity as a surveillance system todetect invasion. Annual Review of Phytopathology 53: 541-563.

de Jonge R, Bolton MD, Thomma BPHJ. 2011. How filamentous pathogens co-opt plants: the ins and outs of fungal effectors. Current Opinion in Plant Biology 14: 400-406.

de Jonge R, Bolton MD, Kombrink A, van den Berg GC, Yadeta KA, Thomma BPHJ. 2013. Extensive chromosomal reshuffling drives evolution of virulence in an asexual pathogen. Genome Research 23: 1271-1282.

de Jonge R, van Esse HP, Maruthachalam K, Bolton MD, Santhanam P, Saber MK, Zhang Z, Usami T, Lievens B, Subbarao KV, Thomma BPHJ. 2012. Tomato immune receptor Ve1 recognizes effector of multiple fungal pathogens uncovered by genome and RNA sequencing. Proceedings of the National Academy of Sciences of the United States of America 109: 5110-5115.

Depotter JR, Deketelaere S, Inderbitzin P, Tiedemann AV, Höfte M, Subbarao KV, Wood TA and Thomma BPHJ. 2016. Verticillium longisporum, the invisible threat to oilseed rape and other brassicaceous plant hosts. Molecular Plant Pathology 17: 1004-1016.

Depotter JRL, Shi-Kunne X, Missonnier H, Liu T, Faino L, van den Berg GCM, Wood TA, Zhang B, Jacques A, Seidl MF, Thomma BPHJ. 2018. Dynamic virulence-related regions of the fungal plant pathogen Verticillium dahliae display remarkably enhanced sequence conservation. bioRxiv doi: https://doi.org/10.1101/277558.

Dong S, Raffaele S, Kamoun S. 2015. The two-speed genomes of filamentous pathogens: waltz with plants. Current Opinion in Genetics and Development 35: 57-65.

Dutheil JY, Mannhaupt G, Schweizer G, MK Sieber C, Münsterkötter M, Güldener U, Schirawski J, Kahmann RA. 2016. A tale of genome compartmentalization: the evolution of virulence clusters in smut fungi. Genome Biology and Evolution 8: 681-704.

Ellendorff U, Fradin EF, De Jonge R, Thomma BPHJ. 2009. RNA silencing is required for Arabidopsis defence against Verticillium wilt disease. Journal of Experimental Botany 60: 591-602.

El-Bebany AF, Rampitsch C, Daayf F. 2010. Proteomic analysis of the phytopathogenic soilborne fungus Verticillium dahliae reveals differential protein expression in isolates that differ in aggressiveness. Proteomics 10: 289-303.

Faino L, Seidl MF, Datema E, van den Berg GC, Janssen A, Wittenberg AH, Thomma BPHJ. 2015. Single-molecule real-time sequencing combined with optical mapping yields completely finished fungal genome. mBio 6: e00936-00915. 
Faino L, Seidl MF, Shi-Kunne X, Pauper M, van den Berg GC, Wittenberg AH, Thomma BPHJ. 2016. Transposons passively and actively contribute to evolution of the twospeed genome of a fungal pathogen.Genome Research 26: 1091-1100.

Faino L, Thomma BPHJ. 2014. Get your high-quality low-cost genome sequence. Trends in Plant Science 19: 288-291.

Fradin EF, Thomma BPHJ. 2006. Physiology and molecular aspects of Verticillium wilt diseases caused by $V$. dahliae and V. albo-atrum. Molecular Plant Pathology 7: 7186.

Fradin EF, Zhang Z, Ayala JCJ, Castroverde CD, Nazar RN, Robb J, Liu C-M, Thomma BPHJ. 2009. Genetic dissection of Verticillium wilt resistance mediated by tomato Ve1. Plant Physiology 150: 320-332.

Frandsen RJ, Andersson JA, Kristensen MB, Giese H. 2008. Efficient four fragment cloning for the construction of vectors for targeted gene replacement in filamentous fungi. BMC Molecular Biology 9: 70.

Friesen TL, Stukenbrock EH, Liu Z, Meinhardt S, Ling H, Faris JD, Rasmussen JB, Solomon PS, McDonald BA, Oliver RP. 2006. Emergence of a new disease as a result of interspecific virulence gene transfer. Nature Genetics 38: 953-956.

Gibriel, H. A. Y., Thomma, B. P. H. J., and Seidl, M. F. 2016. The age of effectors: genomebased discovery and applications. Phytopathology 106: 1206-1212.

Guo S, Zuo Y, Zhang Y, Wu C, Su W, Jin W, Yu H, An Y, Li Q. 2017. Large-scale transcriptome comparison of sunflower genes responsive to Verticillium dahliae. BMC Genomics 18: 42.

Guy L, Kultima JR, Andersson SG. 2010. genoPlotR: comparative gene and genome visualization in R. Bioinformatics 26: 2334-2335.

Holt C, Yandell M (2011) MAKER2: an annotation pipeline and genome-database management tool for second-generation genome projects. BMC Bioinformatics 12: 491.

Inderbitzin P, Subbarao KV. 2014. Verticillium systematics and evolution: how confusion impedes Verticillium wilt management and how to resolve it. Phytopathology 104: 564-574.

Jones JD, Dangl JL. 2006. The plant immune system. Nature 444: 323-329.

Kane NC, Rieseberg LH. 2007. Selective sweeps reveal candidate genes for adaptation to drought and salt tolerance in common sunflower, Helianthus annuus. Genetics 175: 1823-1834.

Klimes A, Dobinson KF, Klosterman SJ, Thomma BPHJ. 2015. Genomics spurs rapid advances in ourunderstanding of the basic biology of vascular wilt pathogens in the genus Verticillium. Annual Review of Phytopathology 53: 181-198.

Klosterman SJ, Atallah ZK, Vallad GE, Subbarao KV. 2009. Diversity, pathogenicity, and management of Verticillium species. Annual Review of Phytopathology 47: 39-62.

Klosterman SJ, Subbarao KV, Kang S, Veronese P, Gold SE, Thomma BPHJ, Chen Z, Henrissat B, Lee YH, Park J. 2011. Comparative genomics yields insights into niche adaptation of plant vascular wilt pathogens. PLoS Pathogens 7: e1002137. 
Kombrink A, Rovenich H, Shi-Kunne X, Rojas-Padilla E, van den Berg G, Domazakis E, De Jonge R,Valkenburg DJ, Sánchez-Vallet A, Seidl MF, Thomma BPHJ. 2017. Verticillium dahliae LysM effectors differentially contribute to virulence on plant hosts. Molecular Plant Pathology 18: 596-608.

Kurtz S, Phillippy A, Delcher AL, Smoot M, Shumway M, Antonescu C, Salzberg SL. 2004. Versatile and open software for comparing large genomes. Genome Biology 5: R12.

Li H, Durbin R. 2010. Fast and accurate long-read alignment with Burrows-Wheeler transform. Bioinformatics 26: 589-595.

Menardo F, Praz CR, Wicker T, Keller B. 2017. Rapid turnover of effectors in grass powdery mildew (Blumeria graminis). BMC Evolutionary Biology 17: 223.

Pedersen C, Ver Loren van Themaat E, McGuffin LJ, Abbott JC, Burgis TA, Barton G, Bindschedler LV, Lu X, Maekawa T, Wessling R, Cramer R, Thordal-Christensen H, Panstruga R, Spanu PD. 2012. Structure and evolution of barley powdery mildew effector candidates. BMC Genomics 13: 694.

Pegg GF, Brady BL. 2002. Verticillium wilts. Wallingord, Oxfordhire: CABI Publishing.

Petersen TN, Brunak S, von Heijne G, Nielsen H. 2011. SignalP 4.0: discriminating signal peptides from transmembrane regions. Nature Methods 8: 785-786.

Plett JM, Daguerre Y, Wittulsky S, Vayssières A, Deveau A, Melton SJ, Kohler A, MorrellFalvey JL, Brun A, Veneault-Fourrey C, Martin F .2014. Effector MiSSP7 of the mutualistic fungus Laccaria bicolor stabilizes the Populus JAZ6 protein and represses jasmonic acid (JA) responsive genes. Proceedings of the National Academy of Sciences of the United States of America 111: 8299-8304.

Plissonneau C, Benevenuto J, Mohd-Assaad N, Fouché S, Hartmann FE, Croll D. 2017. Using population and comparative genomics to understand the genetic basis of effector-driven fungal pathogen evolution. Frontiers in Plant Sciences 8: 119.

Quinlan AR, Hall IM. 2010. BEDTools: a flexible suite of utilities for comparing genomic features. Bioinformatics 26: 841-842.

Rovenich H, Boshoven JC, Thomma BPHJ. 2014. Filamentous pathogen effector functions: of pathogens, hosts and microbiomes. Current Opinion in Plant Biology 20: 96-103.

Rodriguez-Moreno L, Ebert MK, Bolton MD, Thomma BPHJ. 2018. Tools of the crookinfection strategies of fungal plant pathogens. The Plant Journal 93: 664-674.

Sánchez-Vallet A, Fouché S, Fudal I, Hartmann FE, Soyer JL, Tellier A, Croll D. 2018. The genome biology of effector gene evolution in filamentous plant pathogens. Annual Review of Phytopathology 56: 1.

Santhanam P. 2012. Random insertional mutagenesis in fungal genomes to identify virulence factors. Plant Fungal Pathogens Springer (Methods and Protocols) 835: 509-517.

Santhanam P. 2014. Insertional mutagenesis in the vascular wilt pathogen Verticillium dahliae. Wageningen University PhD thesis.

Santhanam P, van Esse HP, Albert I, Faino L, Nürnberger T, Thomma BPHJ. 2013. Evidence forfunctional diversification within a fungal NEP1-like protein family. Molecular Plant-Microbe Interactions 26: 278-286. 
Schaible L, Cannon OS, Waddoups V. 1951. Inheritance of resistance to Verticillium wilt in a tomato cross. Phytopathology 41: 986-990.

Shen D, Liu T, Ye W, Liu L, Liu P, Wu Y, Wang Y, Dou D. 2013. Gene duplication and fragment recombination drive functional diversification of a superfamily of cytoplasmic effectors in Phytophthora sojae. PLoS One 8: e70036.

Simko I, Costanzo S, Haynes K, Christ B, Jones R. 2004. Linkage disequilibrium mapping of a Verticillium dahliae resistance quantitative trait locus in tetraploid potato (Solanum tuberosum) through a candidate gene approach. Theoretical and Applied Genetics 108: 217-224.

Snelders NC, Kettles GJ, Rudd JJ, Thomma BPHJ. 2018. Plant pathogen effector proteins as manipulators of host microbiomes? Molecular Plant Pathology 19: 257-259.

Song Y, Liu L, Wang Y, Valkenburg DJ, Zhang X, Zhu L, Thomma BPHJ. 2018. Transfer of tomato immune receptor Ve1 confers Ave1-dependent Verticillium resistance in tobacco and cotton. Plant Biotechnology Journal 16: 638-648.

Song Y, Thomma BPHJ. 2016. Host-induced gene silencing compromises Verticillium wilt in tomato and Arabidopsis. Molecular Plant Pathology 19: 77-89.

Sperschneider J, Gardiner DM, Dodds PN, Tini F, Covarelli L, Singh KB, Manners JM, Taylor JM. 2016. EffectorP: predicting fungal effector proteins from secretomes using machine learning. New Phytologist 210: 743-761.

Stamatakis, A. 2014. Raxml version 8: a tool for phylogenetic analysis and post-analysis of large phylogenies. Bioinformatics 30: 1312-1313.

Stergiopoulos I, de Wit PJ. 2009. Fungal effector proteins. Annual Review of Phytopathology 47: 233-263.

Stukenbrock EH, Christiansen FB, Hansen TT, Dutheil JY, Schierup MH. 2012. Fusion of two divergent fungal individuals led to the recent emergence of a unique widespread pathogen species. Proceedings of the National Academy of Sciences of the United States of America 109: 10954-10959.

Thomma BP, Nürnberger T, Joosten MH. 2011. Of PAMPs and effectors: the blurred PTIETI dichotomy. Plant Cell 23: 4-15.

van der Does HC, Rep M. 2007. Virulence genes and the evolution of host specificity in plant-pathogenic fungi. Molecular Plant-Microbe Interactions 20: 1175-1182.

Wang M, Weiberg A, Jin H. 2015. Pathogen small RNAs: a new class of effectors for pathogen attacks. Molecular Plant Pathology 16: 219-223.

Wicker T, Oberhaensli S, Parlange F, Buchmann JP, Shatalina M, Roffler S, Ben-David R, Doležel J, Šimková H, Schulze-Lefert P, Spanu PD, Bruggmann R, Amselem J, Quesneville H, Ver Loren van Themaat E, Paape T, Shimizu KK, Keller B. 2013. The wheat powdery mildew genome shows the unique evolution of an obligate biotroph. Nature Genetics 45: 1092-1096.

Yao Z, Rashid KY, Adam LR, Daayf F. 2011. Verticillium dahliae's VdNEP acts both as a plant defense elicitor and a pathogenicity factor in the interaction with Helianthus annuus. Canadian Journal of Plant Pathology 33: 375-388. 
Zhang L, Ni H, Du X, Wang S, Ma XW, Nürnberger T, Guo HS, Hua C. 2017. The Verticilliumspecific protein VdSCP7 localizes to the plant nucleus and modulates immunity to fungal infections. New Phytologist 215: 368-381.

Zhang T, Jin Y, Zhao JH, Gao F, Zhou BJ, Fang YY, Guo HS. 2016. Host-induced gene silencing of the target gene in fungal cells confers effective resistance to the cotton wilt disease pathogen Verticillium dahliae. Molecular Plant 9: 939-942. 


\section{SUPPLEMENTAL INFORMATION}

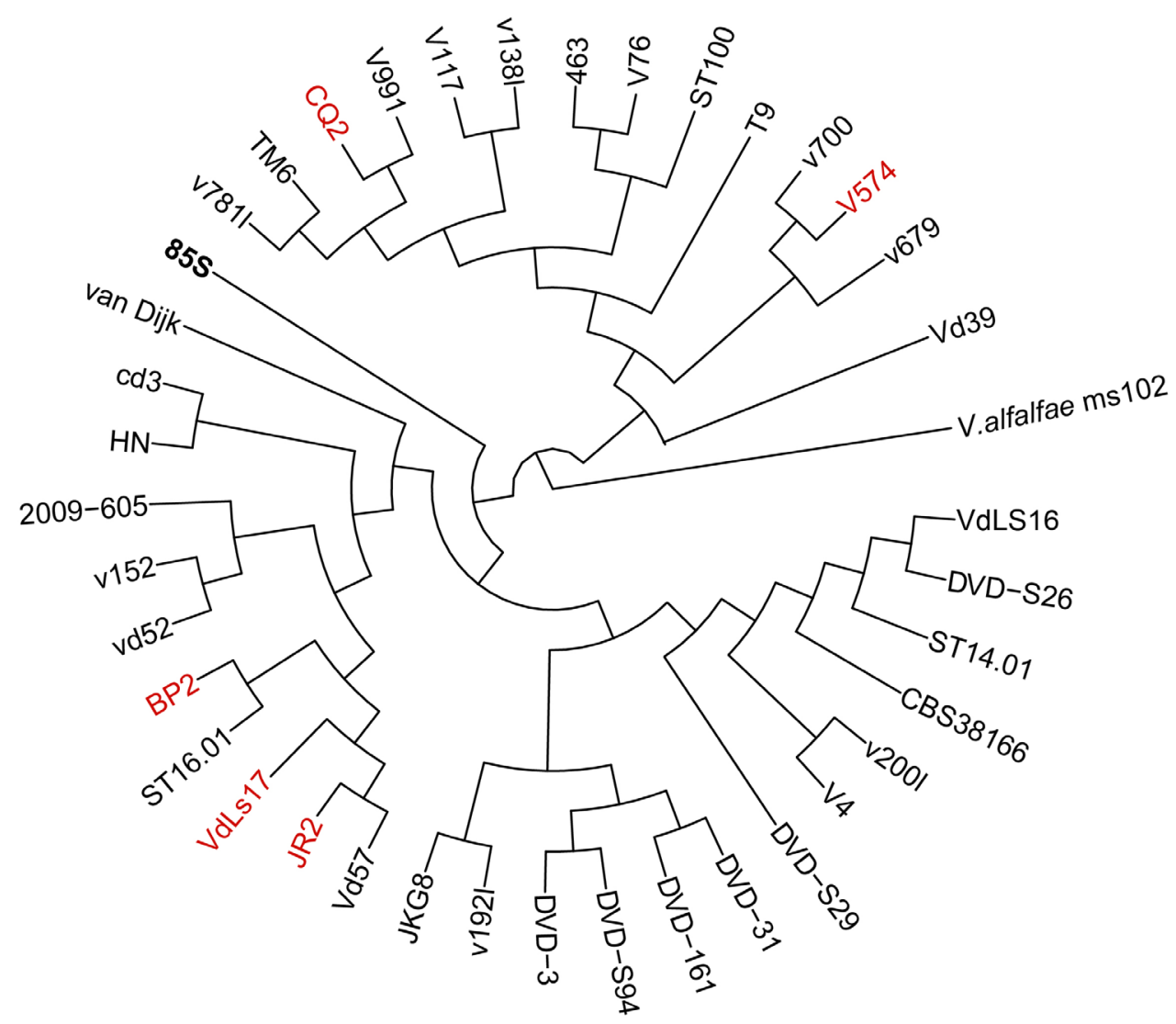

Figure S1. Phylogenetic tree of $\boldsymbol{V}$. dahliae strains.

Sunflower-pathogenic and non-pathogenic strain $85 \mathrm{~S}$ is shown in bold black. Strains that were selected for phenotypic characterization in this study are shown in red font. Phylogenetic relationships between sequenced $V$. dahliae strains are inferred using RealPhy (Bertels et al., 2014). V. alfalfa strain ms102 was used to root the tree. 


\section{A}

\section{Single deletion}

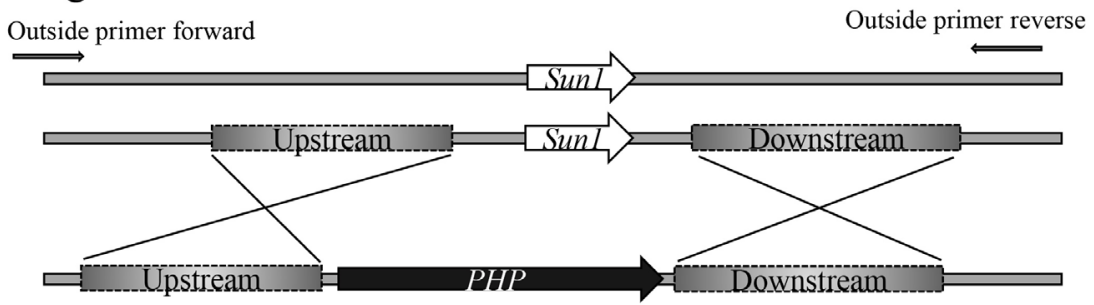

\section{Double deletion}

Outside primer forward Outside primer reverse

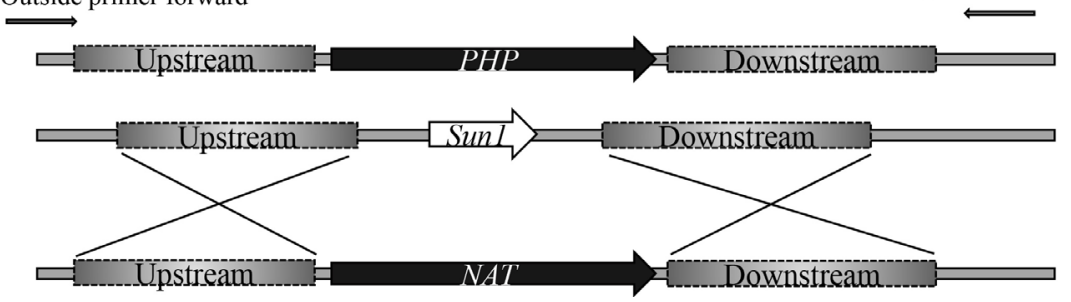

B

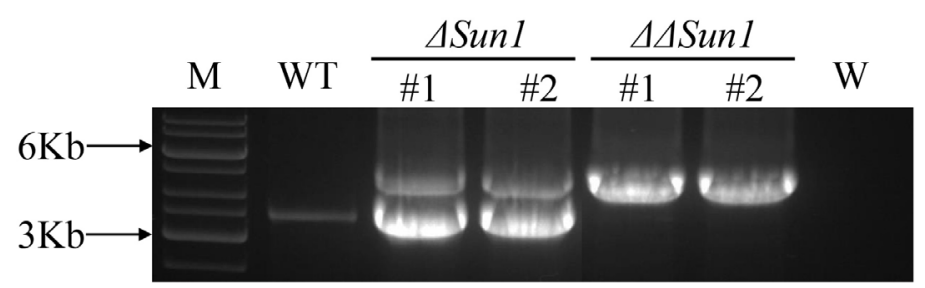

Figure S2. Construction and verification of Sun1 single deletion and double deletion mutants.

(A) Schematic representation of the homologous recombination events to establish targeted replacement of Sun1 with phosphotransferase $(\mathrm{HPH})$ and the nourseothricin resistance gene cassette (NAT). (B) Verification of Sun1 single deletion and double deletion strains by PCR. Amplicons generated with outside primers indicated in panel A are shown for wildtype strain $85 \mathrm{~S}$ (WT), two Sun1 single deletion strains ( $\triangle$ Sun1\#1 and $\triangle$ Sun1\#2) and two Sun1 double deletion strains ( $\triangle \Delta$ Sun1\#1 and $\Delta \Delta$ Sun1\#2). Water was used as negative control (W). 


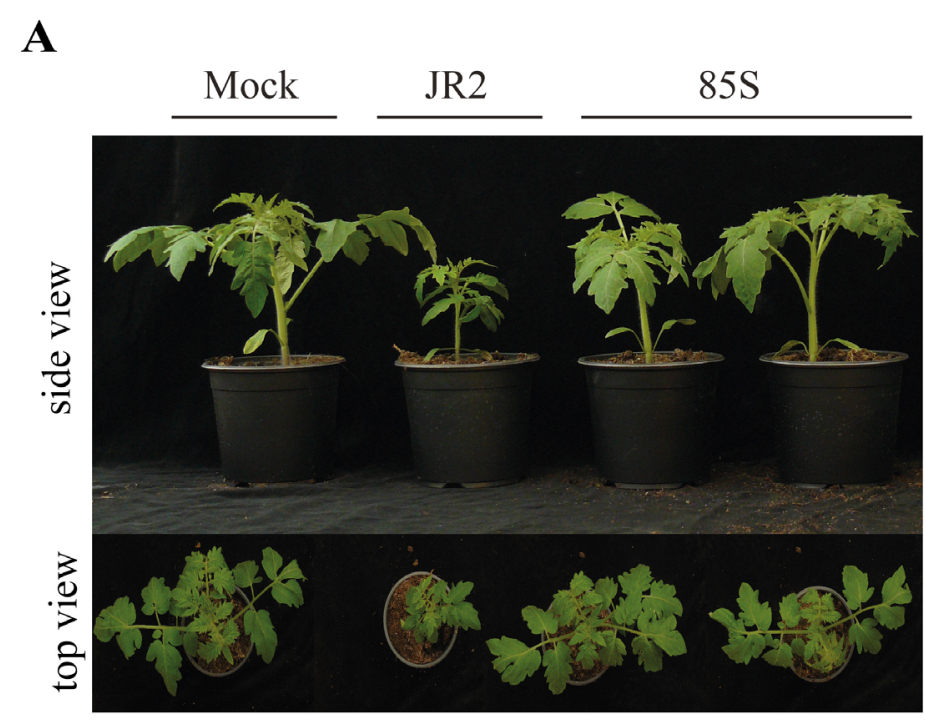

B

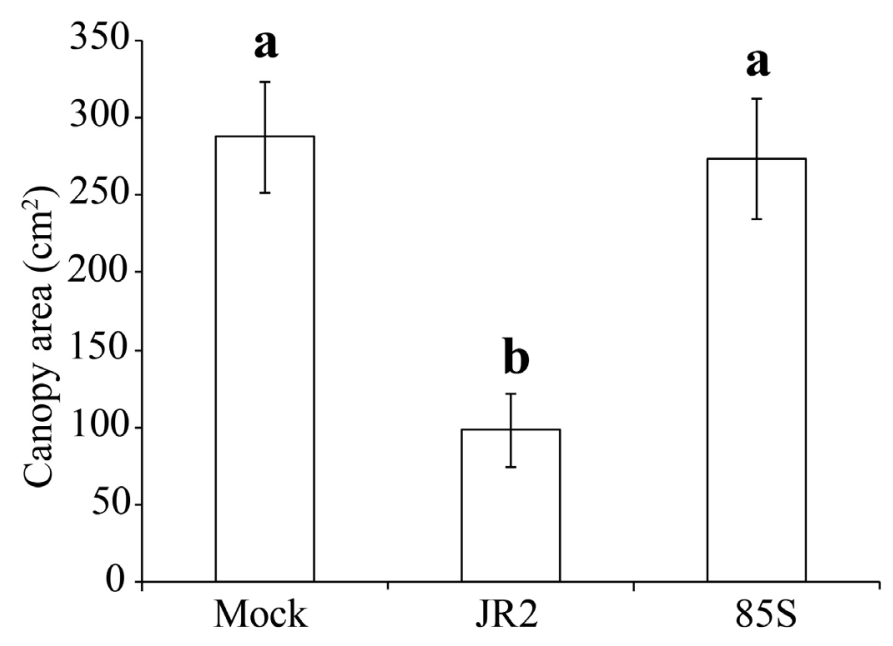

Figure S3. Phenotypes of tomato plants inoculated with $V$. dahliae strain $85 \mathrm{~S}$.

(A) Typical phenotype of tomato(cv. Moneymaker) plants upon mock-inoculation or inoculation with $V$. dahliae strains $85 S$ and JR2 (as positive inoculation control) at 21 days post inoculation (dpi). (B) Quantification of the canopy area of tomato plants at $21 \mathrm{dpi}$. Bars represent average of canopy area of five plants with standard deviation. Different letters indicate statistically significant differences (Student's $t$-test; $P<0.05$ ). Inoculation experiments were performed with five plants for each fungal strain and independently repeated twice with similar results. 

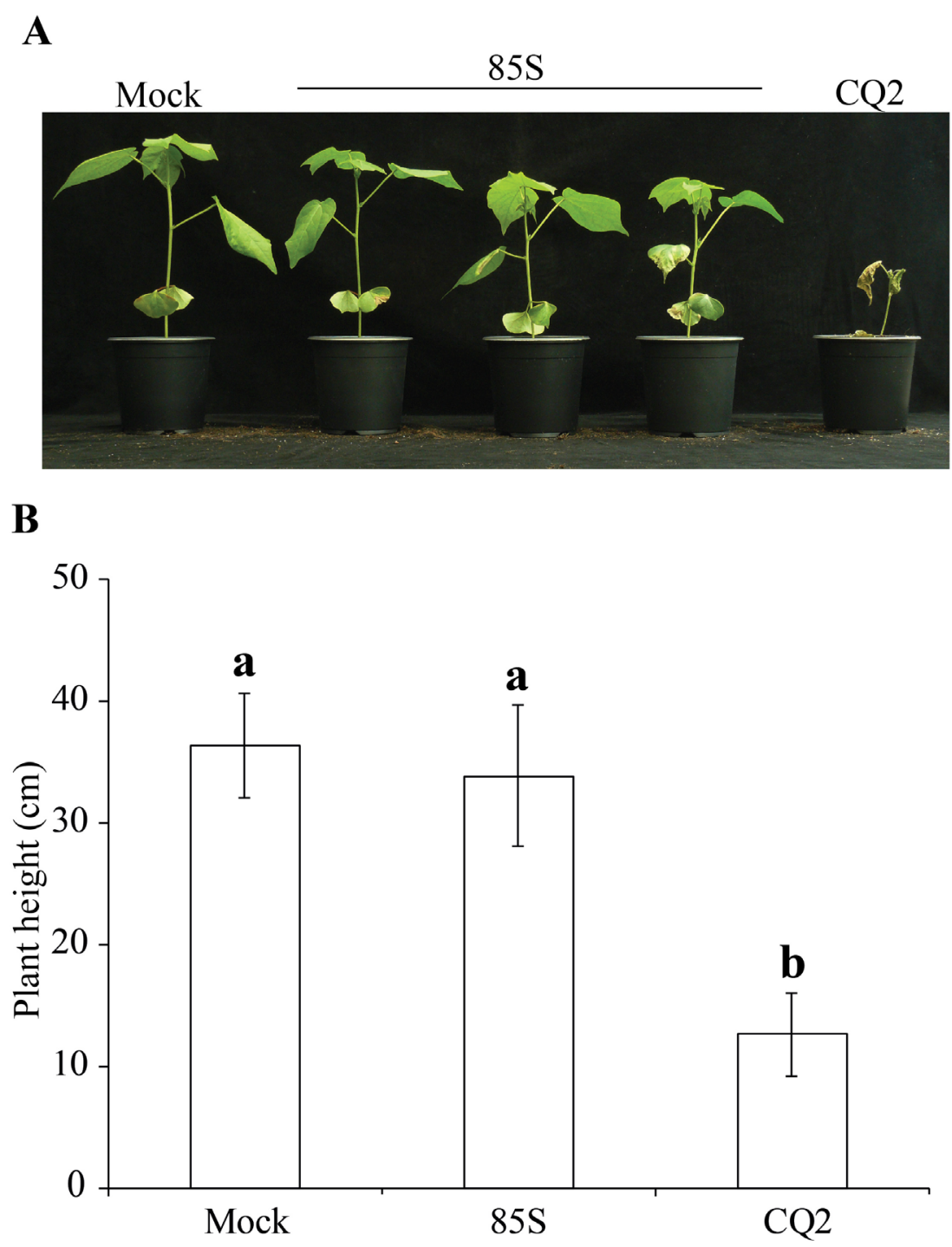

Figure S4. Phenotypes of cotton plants inoculated with $V$. dahliae strain 85S.

(A) Typical phenotype of cotton (cv. Simian3) plants upon mock-inoculation or inoculation with $V$. dahliae strains $85 \mathrm{~S}$ and CQ2 (as positive inoculation control) at 28 days post inoculation (dpi). (B) Quantification of the plant height of cotton plants at $28 \mathrm{dpi}$. Bars represent average of height of five plants with standard deviation. Different letters indicate statistically significant differences (Student's $t$-test; $P<0.05$ ). Inoculation experiments were performed with five plants for each fungal strain and independently repeated twice with similar results. 
Table S1. V. dahliae strains used in this study.

\begin{tabular}{|c|c|c|c|c|}
\hline Strain & $\begin{array}{l}\text { Sequencing } \\
\text { platform }\end{array}$ & Reference & Origin & $\begin{array}{c}\text { Geographical } \\
\text { location }\end{array}$ \\
\hline $\mathrm{HN}$ & Illumina & Xu et al., 2012 & Cotton & China \\
\hline $\mathrm{cd} 3$ & Illumina & Xu et al., 2012 & Cotton & China \\
\hline VdLs17 & PacBio & Faino et al., 2015 & Lettuce & USA \\
\hline$J R 2$ & PacBio & Faino et al., 2015 & Tomato & Canada \\
\hline Vd57 & Illumina & This study & Strawberry & Germany \\
\hline V152 & Illumina & Kombrink et al., 2017 & Oak & Hungary \\
\hline Vd52 & Illumina & Kombrink et al., 2017 & Pepper & Austria \\
\hline van Dijk & Illumina & Kombrink et al., 2017 & Chrysanthemum & $\begin{array}{l}\text { The } \\
\text { Netherlands }\end{array}$ \\
\hline BP2 & Illumina & Zhang et al., 2012 & Cotton & China \\
\hline ST16.01 & Illumina & This study & Cotton & Syria \\
\hline $2009-605$ & Illumina & This study & Bell pepper & Ukraine \\
\hline V4 & Illumina & Keykhasaber, 2017 & Olive & Spain \\
\hline V200I & Illumina & This study & Strawberry & Germany \\
\hline CBS38166 & Illumina & de Jonge et al., 2012 & Tomato & Canada \\
\hline DVD-S26 & Illumina & de Jonge et al., 2012 & Soil & Canada \\
\hline VdLS16 & Illumina & de Jonge et al., 2012 & Lettuce & USA \\
\hline ST14.01 & Illumina & de Jonge et al., 2012 & Pistachio & USA \\
\hline DVD-S29 & Illumina & de Jonge et al., 2012 & Soil & Canada \\
\hline DVD-31 & Illumina & de Jonge et al., 2012 & Tomato & Canada \\
\hline DVD-161 & Illumina & de Jonge et al., 2012 & Tomato & Canada \\
\hline DVD-S94 & Illumina & de Jonge et al., 2012 & Soil & Canada \\
\hline DVD-3 & Illumina & de Jonge et al., 2012 & Potato & Canada \\
\hline V192I & Illumina & This study & Cotton & Spain \\
\hline JKG8 & Illumina & Kombrink et al., 2017 & Potato & $\begin{array}{l}\text { The } \\
\text { Netherlands }\end{array}$ \\
\hline $85 S$ & PacBio & This study & Sunflower & France \\
\hline Vd39 & Illumina & This study & Sunflower & Germany \\
\hline V574 & Illumina & Milgroom et al., 2014 & Artichoke & Spain \\
\hline v700 & Illumina & Milgroom et al., 2014 & Artichoke & Spain \\
\hline v679 & Illumina & Milgroom et al., 2014 & Artichoke & Spain \\
\hline T9 & Illumina & Keykhasaber, 2017 & Cotton & USA \\
\hline V781I & Illumina & This study & Olive & Spain \\
\hline
\end{tabular}


Table S1. continued

\begin{tabular}{ccccc}
\hline Strain & $\begin{array}{c}\text { Sequencing } \\
\text { platform }\end{array}$ & Reference & Origin & $\begin{array}{c}\text { Geographical } \\
\text { location }\end{array}$ \\
\hline V138I & Illumina & This study & Cotton & Spain \\
TM6 & Illumina & Keykhasaber, 2017 & Cotton & China \\
V117 & Illumina & Keykhasaber, 2017 & Olive & Spain \\
V991 & Illumina & Zhang et al., 2012 & Cotton & China \\
CQ2 & PacBio & This study & Cotton & China \\
ST100 & Illumina & de Jonge et al., 2012 & Soil & Belgium \\
V76 & Illumina & This study & Cotton & Mexico \\
463 & Illumina & This study & Cotton & Mexico \\
\hline
\end{tabular}

Table S2. Primers used in this study.

\begin{tabular}{|c|c|c|}
\hline Primer name & Oligonucleotide sequence $\left(5^{\prime} \rightarrow 3^{\prime}\right)^{\mathrm{a}}$ & Description \\
\hline ITS-F & AAAGTTTTAATGGTTCGCTAAGA & $\begin{array}{l}\text { Verticillium ribosomal internal } \\
\text { transcribed spacer region } \\
\text { (ITS), fungal biomass }\end{array}$ \\
\hline ITS-R & CTTGGTCATTTAGAGGAAGTAA & $\begin{array}{l}\text { Verticillium ribosomal internal } \\
\text { transcribed spacer region } \\
\text { (ITS), fungal biomass }\end{array}$ \\
\hline SKO-Sun1-LF & GGTCTTAAUCAGATACCGATTATTGATCCTCGAC & $\begin{array}{c}\text { For single Sun1 deletion } \\
\text { generation }\end{array}$ \\
\hline SKO-Sun1-LR & GGCATTAAUCGTTAAGAGTTCATAGGCGAAGTTA & $\begin{array}{c}\text { For single Sun } 1 \text { deletion } \\
\text { generation }\end{array}$ \\
\hline SKO-Sun1-RF & GGACTTAAUCTCGAAATTACAGAGCTTGCTATGA & $\begin{array}{c}\text { For single Sun1 deletion } \\
\text { generation }\end{array}$ \\
\hline SKO-Sun1-RB & GGGTTTAAUACTTGGCTATTTCTTCGTCTTTAGG & $\begin{array}{c}\text { For single Sun1 deletion } \\
\text { generation }\end{array}$ \\
\hline DKO-Sun1-LF & GGTCTTAAUTAGATTGTGTCCTGTGCAAGATATG & $\begin{array}{c}\text { For double Sun1 deletion } \\
\text { generation }\end{array}$ \\
\hline DKO-Sun1-LR & GGCATTAAUAGGGTTAACGTACATTATCAGCATG & $\begin{array}{c}\text { For double Sun } 1 \text { deletion } \\
\text { generation }\end{array}$ \\
\hline DKO-Sun1-RF & GGACTTAAUGATCCCCTTGTCCATTATCTAGTGA & $\begin{array}{c}\text { For double Sun } 1 \text { deletion } \\
\text { generation }\end{array}$ \\
\hline DKO-Sun1-RB & GGGTTTAAUCCGGGACAAAGGAAGGTTAATATAC & $\begin{array}{c}\text { For double Sun } 1 \text { deletion } \\
\text { generation }\end{array}$ \\
\hline
\end{tabular}


Table S2. continued

\begin{tabular}{|c|c|c|}
\hline Primer name & Oligonucleotide sequence $\left(5^{\prime} \rightarrow 3^{\prime}\right)^{\mathrm{a}}$ & Description \\
\hline $\begin{array}{l}\text { outside } \\
\text { primer-F }\end{array}$ & GCCTCACAACCAATCCACAG & $\begin{array}{l}\text { Verification of Sun1 deletion } \\
\text { mutants }\end{array}$ \\
\hline $\begin{array}{l}\text { outside } \\
\text { primer-R }\end{array}$ & ACATCGCCTCAGAGTCACAA & $\begin{array}{l}\text { Verification of Sun1 deletion } \\
\text { mutants }\end{array}$ \\
\hline Sun1-F (RT) & СТСАTACTCTCCTCCGGTTCAT & Sun1, RT-PCR \\
\hline Sun1-R (RT) & TTGTACCATCTCCACACGTTAAGTA & Sun1, RT-PCR \\
\hline VdGAPDH-F & CGAGTCCACTGGTGTCTTCA & V. dahliae GAPDH, RT-PCR \\
\hline VdGAPDH-F & CCCTCAACGATGGTGAACTT & V. dahliae GAPDH, RT-PCR \\
\hline Ha-ELF-1 $1 \alpha-F$ & ACCAAATCAATGAGCCCAAG & $\begin{array}{l}\text { Sunflower elongation Factor } \\
1-\alpha(\text { Ha-EF-1 } \alpha) \text {, fungal biomass }\end{array}$ \\
\hline Ha-ELF-1 $\alpha-R$ & GAGACTCGTGGTGCATCTCA & $\begin{array}{l}\text { Sunflower elongation Factor } \\
1-\alpha(\text { Ha-EF-1 } 1 \alpha) \text {, fungal biomass }\end{array}$ \\
\hline AtRubisco-F & GCAAGTGTTGGGTTCAAAGCTGGTG & $\begin{array}{c}\text { Arabidopsis Rubisco, fungal } \\
\text { biomass }\end{array}$ \\
\hline AtRubisco-R & CCAGGTTGAGGAGTTACTCGGAATGCTG & $\begin{array}{c}\text { Arabidopsis Rubisco, fungal } \\
\text { biomass }\end{array}$ \\
\hline NbRubisco-F & TCCGGGTATTAGGAAAAGCGT & $\begin{array}{c}\text { N. benthamiana Rubisco, } \\
\text { fungal biomass }\end{array}$ \\
\hline NbRubisco-R & CCCAAGATCTGGGTCAGAGC & $\begin{array}{c}\text { N. benthamiana Rubisco, } \\
\text { fungal biomass }\end{array}$ \\
\hline
\end{tabular}

${ }^{\text {a } U S E R ~ c l o n i n g ~ s i t e s ~ p r e s e n t ~ i n ~ p r i m e r ~ s e q u e n c e ~ a r e ~ u n d e r l i n e d, ~ R T-P C R, ~ r e a l-t i m e ~ P C R . ~}$ 


\section{Chapters}

$$
\text { do }
$$

$$
26
$$

ᄂ 


\section{Signatures of adaptation to bread or durum wheat in a global collection of Zymoseptoria tritici isolates}

Hesham A.Y. Gibriel ${ }^{1,2}$, Guillaume Robert-Siegwald ${ }^{3}$, Anne Genissel ${ }^{3}$, Els C.P. Verstappen ${ }^{4}$, L.C. Paul Keizer ${ }^{4}$, Steven B. Goodwin ${ }^{5}$ Marc H. Lebrun $^{3}$, Gert H.J. Kema ${ }^{1,4 *}$ and Michael F. Seidl ${ }^{1 \#^{*}}$

1 Laboratory of Phytopathology, Wageningen University and Research, Droevendaalsesteeg 1, 6708 PB Wageningen, The Netherlands.

2 Current address: School of Agriculture and Food Science, University College Dublin, Dublin, Ireland.

3 UMR BIOGER, Institut National de la Recherche Agronomique (INRA), Thiverval-Grignon, France.

4 Wageningen Plant Research, Wageningen University and Research, Wageningen, the Netherlands.

5 USDA-Agricultural Research Service, Purdue University, West Lafayette, Indiana, United States of America.

\# These authors contributed equally

To be submitted for publication 


\begin{abstract}
Zymoseptoria tritici is a sexually reproducing fungal pathogen and is a major threat for wheat production in Europe and worldwide. The fungus exhibits host specificity, as it generally infects either bread wheat (BW; Triticum aestivum) or durum wheat (DW; Triticum turgidum), as well as cultivar specificity. By combining whole-genome sequencing of a worldwide collection of $136 \mathrm{Z}$. tritici isolates and phenotyping assays on a set of BW and DW cultivars, we observed extensive genetic diversity with moderate genetic differentiation among the geographical groups. Isolates originating from the Middle East, wheat's centre of origin, displayed increased genetic diversity when compared with populations from other geographical locations. We show large-scale structural genome variations and confirm the presence of 13 core chromosomes harbouring most of the identified effector genes, and structurally variable accessory chromosomes with higher SNP rates and presence/absence polymorphisms among effector genes. Profiling these effectors revealed a group of 183 conserved effectors and a group of 88 effectors that show presence/absence polymorphisms that were significantly closer to transposable elements than conserved ones. Assessment of genome-wide differences between BW and DW isolates revealed four effector genes affected by non-synonymous single nucleotide polymorphisms in BW isolates. Future functional analyses are needed to understand the role of the identified effector candidates on BW or DW cultivars.
\end{abstract}




\section{INTRODUCTION}

Microbial plant pathogens cause devastating diseases, thereby threatening global food security (Fisher et al. 2012). However, the majority of microbes is harmless to plants that have evolved an immune system that is able to detect pathogen invasions and mount appropriate defence responses (Cook et al. 2015). To overcome plant immunity, pathogens secrete effector proteins to enable host colonization, often by deregulation of plant immune responses (Cook et al. 2015; Rovenich et al. 2014). In turn, plants evolved immune receptors that can recognize such effectors leading to the re-establishment of immunity (Cook et al. 2015). This poses a selection pressure driving the emergence of favourable mutations in pathogen populations to evade such recognition (Cook et al. 2015; Möller and Stukenbrock 2017) that, in a continuous co-evolution, leads to extensive genomic diversity in plant and pathogen populations.

To facilitate co-evolution with their hosts, many plant pathogens evolved a compartmentalized genome with gene-dense and gene-sparse regions (Dong et al. 2015; Raffaele and Kamoun 2012). Gene-sparse regions often contain effector genes and display signs of accelerated evolution (Dong et al. 2015; Raffaele and Kamoun 2012; Seidl and Thomma 2017). For example, effector genes of the potato late blight pathogen Phytophthora infestans are located in gene-sparse, repeat-rich regions that are characterized by extensive structural variation and increased effector gene diversity (Haas et al. 2009; Raffaele et al. 2010). In the vascular wilt pathogen Verticillium dahliae, in planta expressed effector genes are localized in repeat-rich, and thus relatively genesparse, lineage-specific (LS) regions that evolved by chromosomal rearrangements (de Jonge et al. 2013; Faino et al. 2016). Gene-sparse genomic regions are either located on the core chromosomes, or can reside on separate chromosomes that are often referred to as dispensable or accessory chromosomes (Croll et al. 2013; de Jonge et al. 2013; Faino et al. 2016; Raffaele and Kamoun 2012; Wittenberg et al. 2009).

Wheat is currently the third main human food crop after maize and rice (FAOSTAT 2015), and the production is dominated by bread wheat (BW) (Triticum aestivum) and durum wheat (DW) (Triticum turgidum) (Troccoli et al. 2000). However, the production of wheat is continuously theatened by various plant pathogens such as cereal rusts (Hubbard et al. 2015; Singh et al. 2011), and Zymoseptoria tritici that causes septoria tritici leaf blotch (STB), which is a major threat for wheat production worldwide (Kettles and Kanyuka 2016; O'Driscoll et al. 2014). The fungus shows clear host specificity, as individual isolates originating from DW are virulent on the majority of DW cultivars, yet generally avirulent on BW cultivars, and vice versa (Mirzadi Gohari et al. 2015). Additionally, Z. tritici isolates display cultivar specificity (Kema et al. 1996; Mirzadi Gohari et al. 2015), which is mediated by the presence of specific resistance genes in different cultivars. Thus far, 21 resistance genes, so called septoria tritici blotch (Stb) genes, have been genetically mapped (Brown et al. 2015), of which Stb6 (Chartrain et 
al. 2005a), as well as the corresponding avirulence effector gene AvrStb6 were recently cloned (Chapter 6) (Kema et al. 2018; Saintenac et al. 2018; Zhong et al. 2017).

Sexual reproduction is an intricate part of the life cycle of Z. tritici (Kema et al. 1996; Wittenberg et al. 2009), which results in highly polymorphic populations (Hartmann et al. 2018; Hartmann et al. 2017; McDonald et al. 2016; Naouari et al. 2016; Stukenbrock et al. 2011; Zhan et al. 2003). The reference Z. tritici isolate IPO323 harbours 21 chromosomes, comprising 13 core chromosomes that are present in all studied isolates, and eight dispensable or accessory chromosomes that can be absent without obvious fitness effects (Wittenberg et al. 2009). Particularly the accessory chromosomes are highly dynamic, showing abundant genomic rearrangements and extensive length variations, as well as presence/absence polymorphisms (Croll et al. 2013; Habig et al. 2017; Stukenbrock et al. 2010; Wittenberg et al. 2009). These chromosomes are generally gene-poor and can undergo fusions. Taken together, these processes likely contribute to rapid adaptive evolution of Z. tritici (Croll et al. 2013; Fouché et al. 2018b). Interestingly, thus far, no effector genes were identified on $Z$. tritici accessory chromosomes (McDonald et al. 2015; Mirzadi Gohari et al. 2015). The previously characterized Z. tritici effectors Mg3LysM and Mg1LysM, which belong to a conserved group of LysM effectors that are found in many fungal species (de Jonge and Thomma 2009; Kombrink et al. 2016; Kombrink and Thomma 2013), are located on core chromosomes (Marshall et al. 2011). Both Mg1LysM and Mg3LysM can bind chitin and protect fungal hyphae against plant hydrolytic enzymes, while Mg3LysM also blocks the activation of chitin-induced host immunity (Marshall et al. 2011). Similarly, the other known effector genes AvrStb6 (Kema et al. 2018; Zhong et al. 2017), Zt_8_609 (Hartmann et al. 2017), Avr3D1 (Meile et al. 2018b) and Zt80707 (Poppe et al. 2015) are located on core chromosomes, usually in orphan genomic regions associated with transposable elements (TEs) or recombination hotspots (Croll et al. 2015; Meile et al. 2018b; Plissonneau et al. 2016). For example, genome-wide analyses revealed that AvrStb6 resides in a recombination hotspot, which exhibits signatures of accelerated evolution including elevated SNP levels (Brunner and McDonald 2018; Kema et al. 2018; Zhong et al. 2017). Taken together, the natural diversification of $Z$. tritici effectors suggests a continuous co-evolutionary adaptation of pathogen populations to their wheat hosts.

Population genomics is a powerful method that can improve our understanding of diversity in natural pathogen populations and estimate their evolutionary potential (Grünwald et al. 2016). Initial studies showed genetic diversification in local and global populations, with high rates of gene flow and limited population structure (Linde et al. 2002; Zhan et al. 2003). These studies were based on a limited number of molecular markers, which likely resulted in a biased assessment of the overall genomic diversity of this species and could not reveal loci associated with Z. tritici cultivar specificity (McDonald et al. 2015). Recent population genomics studies further explored the genetic diversity of Z. tritici populations (Grandaubert et al. 2018; Hartmann et al. 
2018; Hartmann et al. 2017; McDonald et al. 2016). Although these studies often assessed Z. tritici isolates that were not widely geographically separated and were often sampled from single wheat fields (Hartmann et al. 2018; Hartmann et al. 2017), they corroborated previous findings that Z. tritici is a highly dynamic pathogen that genetically differs between and within populations, even at small spatial scales (Croll et al. 2013; Hartmann et al. 2017; Linde et al. 2002; McDonald et al. 2016; Stukenbrock et al. 2011). Here, we aimed to explore the genomic diversity in 136 Z. tritici isolates that were sampled from bread and durum wheat accessions in 31 locations in the major global wheat producing regions. By comparing the chromosome and effector diversity of BW and DW isolates, along with the phenotyping of 118 isolates on four wheat cultivars, we provide a foundation for further genomic and functional studies of host specificity in Z. tritici.

\section{MATERIALS AND METHODS}

\section{Fungal isolates and phenotyping assays}

In total, 136 Z. tritici isolates were sampled from single wheat fields at 31 locations (Table S1). Upon sampling, collected isolates were prepared for long-term storage at $-80^{\circ} \mathrm{C}$ (Kema et al. 1996).

Phenotyping assays were conducted on the bread wheat cvs. Taichung 29 (susceptible BW control; Kema et al. 1996), Bulgaria 88 (carries Stb1 and Stb6; (Adhikari et al. 2004; Chartrain et al. 2005a), TE9111 (carries Stb11, Stb7, and Stb11; (Chartrain et al. 2005b) and the DW cv. Volcani 447 (susceptible control; Kema et al. 1996; Mirzadi Gohari et al. 2015). Wheat seeds were planted in potting soil (Swedish sphagnum peat $5 \%$, grinding clay granules $41 \%$, garden peat $5 \%$, beam structure $4 \%$, steamed 140 compost $33 \%$, PG-Mix-15-10-20-12\%) from the Unifarm greenhouse facility of Wageningen University and Research, Wageningen, The Netherlands, and after germination plants were grown until inoculation for 10 days in the greenhouse at $18^{\circ} \mathrm{C}$, under $16 \mathrm{~h}$ light and 8 $\mathrm{h}$ dark cycles and a relative humidity (RH) of $75 \%$ (Kema et al. 2018). The isolates were taken from the $-80^{\circ} \mathrm{C}$ stocks, cultured for 5-10 days on potato dextrose agar and from there transferred to yeast-glucose medium (yeast extract $10 \mathrm{~g} / \mathrm{L}$, glucose $30 \mathrm{~g} / \mathrm{L}$ ) and incubated in an orbital shaker (New Brunschwick (now Eppendorf) Innova 44r, orbit diameter $2.5 \mathrm{~cm}$, The Netherlands) for $4-6$ days at $16^{\circ} \mathrm{C}$. Yeast-like conidiospores were collected by overnight sedimentation and inoculum was adjusted at $10^{7}$ conidiospores/ $\mathrm{mL}$ supplemented with two drops of Tween 20 (Merck, The Netherlands) surfactant and atomized over the primary leaves of the wheat seedlings. After inoculation, plants were kept in transparent plastic bags at $22^{\circ} \mathrm{C}$ at $100 \%$ relative humidity $(\mathrm{RH})$ for $48 \mathrm{~h}$, and were subsequently maintained in the same greenhouse at RH 95\%. Disease development was monitored daily and after 10 days secondary and subsequent leaves were removed to ensure sufficient light on the inoculated leaves. After 21 days the inoculated leaves were collected and the percentage of leaf necrosis and the percentage of leaf area covered 
with pycnidia were assessed on a 0-100 scale. We used a split plot statistical design and all assays were performed in two biological replicates with the $Z$. tritici isolates IPO87016.MS1, IPO-90006.MS2, and a mock present in each tray as controls. The allocation of isolates over the trays was randomized, but both biological replicates were always in different blocks.

\section{DNA extraction and whole-genome sequencing}

Genomic DNA of all Z. tritici isolates was extracted from five-day-old liquid cultures grown in yeast extract-peptone-dextrose broth (YPD) using a standard phenolchloroform procedure (Sambrook et al. 1989). Library preparation ( 500 bp insert size) and whole-genome sequencing (150 bp paired-end reads) using Illumina HiSeq-2500 sequencing were performed at the Joint Genome Institute (JGI, Walnut Creek, CA, USA) and the generated raw sequencing data are deposited at the JGI under project CSP983.

\section{Single nucleotide polymorphisms (SNPs) calling}

We generated a repeat-masked genome of Z. tritici IPO323 (Goodwin et al. 2011) and used it as a reference for all subsequent analyses. To this end, de novo repeats were annotated using REPET (v2.2; default setting) (Flutre et al. 2010), which was further utilized to subsequently mask the genome sequence using RepeatMasker (v4.0.6) (Smit et al. 2016).

Paired-end reads of each isolate were mapped to the masked reference genome $Z$. tritici IPO323 (Goodwin et al. 2011) using BWA (MEM) (default options) (v0.7) (Li and Durbin 2010). PCR duplicates were marked using Picard tools (v1.1) (http://broadinstitute. github.io/picard). Single nucleotide polymorphisms (SNPs) were identified using the HaplotypeCaller of the Genome Analysis Toolkit (GATK) (v3.8) (McKenna et al. 2010). First, variations were detected for each isolate individually with the following settings: emitRefConfidence GVCF and ploidy 1. Joint genotyping for all isolates was performed using GenotypeGVCFs with the option maxAltAlleles 2. All non-SNPs variants were removed using SelectVariants (-selectType SNP) and to obtain high quality SNPs, they were further filtered using VariantFiltration with the following cut-offs: QUAL $>250$, $\mathrm{MQ}>30, \mathrm{QD}>20, \mathrm{FS}<0.1$, and BaseQRankSum, ReadPosRankSum, MQRankSumPos between -2 and 2 . Next, we excluded SNPs with missing genotype calls in $10 \%$ of the isolates and a minor allele frequency (MAF) $<5 \%$ using vcftools (v0.1.5) (Danecek et al. 2011), similar to the procedure of Hartmann et al. (2017). Moreover, we excluded SNPs on the accessory chromosomes, as these are not conserved across the global panel (Goodwin et al. 2011). Principal component analysis (PCA), using the filtered SNP set, was performed with SNPRELATE (v1.6) (Zheng et al. 2012). SNPs were annotated with SnpEff (v3.2) (Cingolani et al. 2012), using the manually-refined gene annotation of Z. tritici isolate IPO323 (Kema et al. 2018). A phylogenetic tree was constructed using RealPhy (readLength 150) (Bertels et al. 2014) that used Bowtie2 (Langmead and Salzberg 2012) to map reads of individual $Z$. tritici isolates to the repeat-masked genome of Z. tritici IPO323 (Goodwin et al. 2011). The population structure was analysed using 
Structure (v.2.3.2) (Falush et al. 2003; Pritchard et al. 2000) and the data were analysed with $\mathrm{K}$ ranging from one to eight with ten repetitions for each tested $\mathrm{K}$ value. We used 50,000 samples as a burn-in period and 100,000 samples per run for the Monte Carlo Markov Chain (MCMC) replicates.

\section{Chromosomes and effector polymorphisms}

Whole-genome sequencing data were used to determine chromosome polymorphisms across the entire panel. To this end, the read coverage of each isolate relative to the masked reference genome IPO323 was determined using BEDtools coverage (v2.25) (default parameters) (Quinlan and Hall, 2010). Subsequently, the individual read coverages for each isolate were normalized by averaging the read coverage for each chromosome over the whole-genome coverage and boxplots were produced using the R package ggplot2 (Wickham 2015). Next, we determined chromosome polymorphisms following the procedure of Fouché et al. (2018) by classifying a chromosome as (i) absent, if the normalized coverage ratio is close to zero $(<0.3)$, (ii) present, if the normalized coverage ratio is close to one ( $\geq 0.7$ and $<1.3$ ), (iii) duplicated, if the normalized coverage ratio is close to two ( $\geq 1.7$ ), (iv) partially deleted, if the normalized coverage ratio is $\geq 0.3$ and $<0.7$, or as (v) partially duplicated, if the normalized coverage ratio is $\geq 1.3$ and $<1.7$. Heatmaps indicating the chromosome polymorphisms were generated using the $\mathrm{R}$ package pheatmap (Kolde and Kolde 2015). In addition, we carried out Pulsed field gel electrophoresis (PFGE), as described by (Mehrabi et al. 2007), for Z tritici isolates IPO323 and ND95 to check whether the overall chromosome polymorphisms match with the whole-genome sequencing data.

To identify Z. tritici candidate effectors, N-terminal signal peptides were first predicted for all the manually-refined protein annotations of Z . tritici isolate IPO323 (Kema et al. 2018) using SignalP (v4.1) (Petersen et al. 2011). Subsequently, effector candidates were predicted from the set of secreted proteins using the machine-learning approach applied in EffectorP (v1.0) (default parameters) (Sperschneider et al. 2016). The effector polymorphisms across the entire global panel in relation to Z. tritici IPO323 were determined using BEDtools coverage (Quinlan and Hall 2010). To assess effector gene expression levels, we used an RNA-seq dataset (single-end) of Z. tritici isolate IPO323 collected over the entire pathogenesis on wheat cv. Riband (Rudd et al. 2015). RNAseq data were mapped to the reference $Z$. tritici genome IPO323 using Tophat (v2.0) (min-intron-length 20, max-intron-length 2000, max-multihits 5) (Dobin et al. 2013). Subsequently, gene expression levels for each sample were estimated using Cuffdiff (v2.2) with default parameters (Trapnell et al. 2010) and reported as fragments per kilobase of exon per million fragments mapped (FPKM). 


\section{RESULTS}

\section{The global panel of Zymoseptoria tritici is highly polymorphic}

Our collection comprised $136 Z$. tritici isolates sampled from BW (95) or DW (14) and 27 isolates were sampled from unknown wheat accessions (Table S1). Most countries were represented by five (variable between 3-8) Z. tritici isolates, whereas some were represented by a single isolate, such as Bolivia, Peru, Italy and Romania, or more isolates (15-21) for France, Iran and the USA (Table S1). The isolates were grouped into seven distinct populations based on their geographical origin (North America, South America, South Europe and the North African countries Morocco, Tunisia and Algeria - collectively known as the Maghreb- East Africa, Middle East, North Europe, and Oceania) (Figure $1 \mathrm{~A}$; Table S1).

To estimate the genetic diversity across the collective panel, each isolate was genotyped and in total 877,279 bi-allelic SNPs were identified, with on average 379,000 SNPs per Z. tritici isolate, indicating high levels of genetic diversity. The Oceania population displayed the least number of SNPs (average of 9.4 SNPs per kb) compared with the other populations (Figure 1B). The Middle East population showed the highest number of SNPs (average of 23.8 SNPs per kb), highlighting that genetic diversity in Z. tritici populations significantly varies between different geographical regions and that the maximum genetic diversity is observed in the Middle East population with an average number of 440,977 SNPs per isolate (ranging from 413,284 to 528,164 SNPs) (Figure $1 B)$.

To analyse the population structure of the global panel, we performed a principal component analysis (PCA) using all identified SNPs, and reconstructed a maximumlikelihood phylogeny based on 291,000 biallelic SNPs as determined by RealPhy (Bertels et al. 2014). We observed that $Z$. tritici isolates are highly polymorphic and do not consistently group by geographical origin (Figure $1 \mathrm{C}$ and $\mathrm{D}$ ). We therefore performed an additional population genetic analysis and observed a geographical pattern for the populations from North Europe, North America, and the Middle-East (Figure 1C), suggesting a moderate genetic differentiation at a global scale. 
Figure 1. Highly diverse global Zymoseptoria tritici isolates. (A) The number of isolates sampled in seven color-coded major wheat producing regions of the world. (B) The number of identified single nucleotide polymorphisms in all $Z$. tritici isolates in each population. The blue horizontal lines represent the average number of SNPs for each population. (C) Principal component analysis (PCA) based on all identified 877,279 SNPs in all Z. tritici populations (upper panel). The percentage of variance explained by each component is shown in parentheses. Lower panel shows the clustering assignments of each $Z$. tritici genotype inferred using the software Structure. The distribution of individual assignations estimated for $\mathrm{K}=7$ clusters, from Bayesian inference cluster analysis performed with 9,874 Z. tritici SNPs. Each vertical line represents an individual isolate. (D) Phylogenetic tree of all Z. tritici isolates based on 291,000 SNPs. Colored nodes correspond to the geographic origins of the $Z$. tritici isolates.
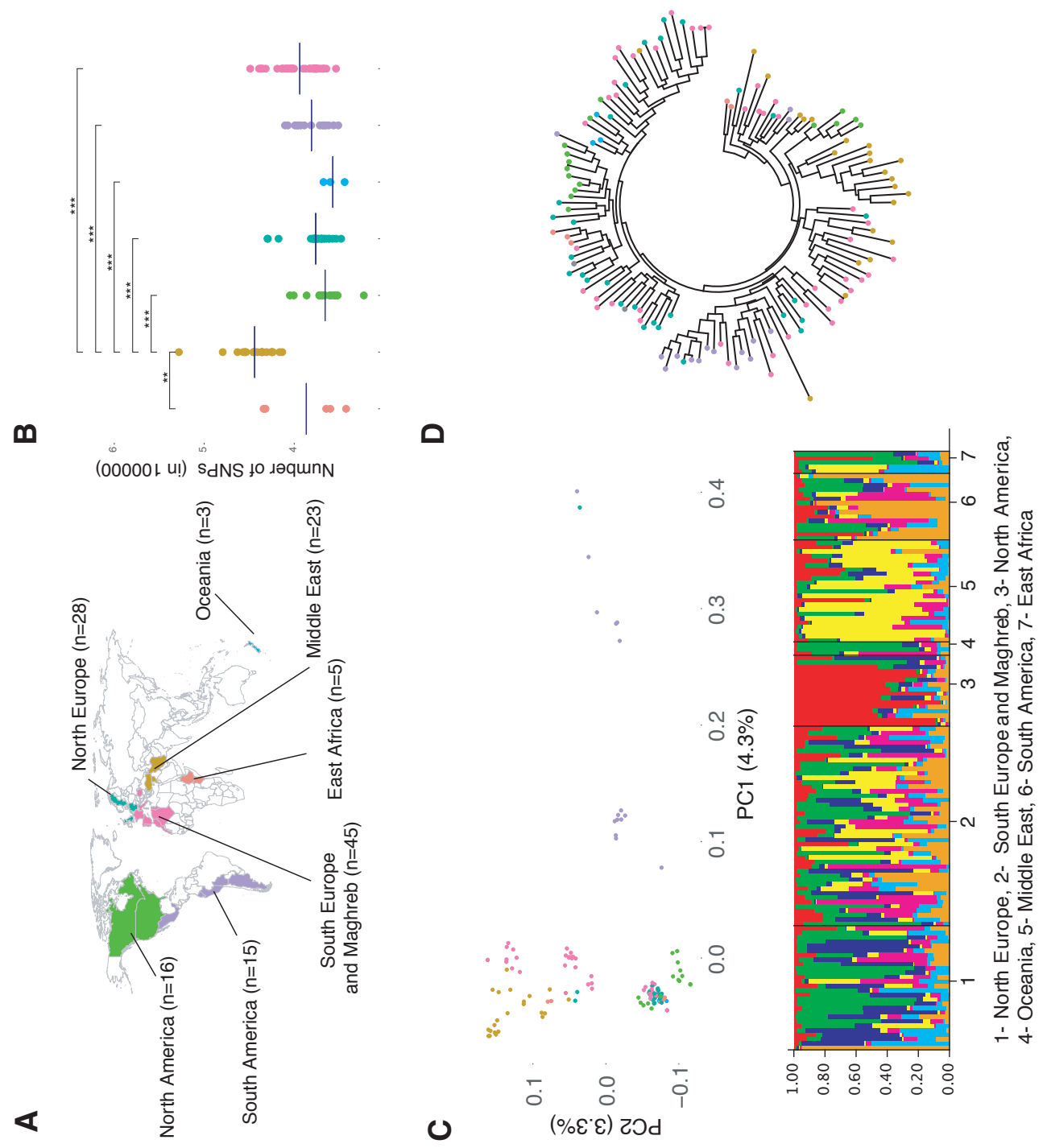


\section{The highly polymorphic accessory chromosomes of Zymoseptoria tritici}

The $Z$. tritici reference isolate IPO323 contains 21 chromosomes comprising 13 core chromosomes and eight accessory chromosomes (Goodwin et al. 2011; Habig et al. 2017; Wittenberg et al. 2009). We assessed chromosome size and number polymorphisms across the entire panel and observed that all core chromosomes are always present and only display size polymorphisms (Figure 2A and B). However, we observed a few cases of partial chromosomal duplications in isolates IPO-10014 (duplication of chromosome 12), IPO-92044 (duplication of chromosome 4), and IPO-98114, IPO-10013, IPO-95001, and IPO-92044 (duplication of chromosome 5) (Figure 2A). In addition, we observed partial duplication of chromosome 7 in isolates IPO-09007, IPO-98047, and IPO-10012 and also of chromosome 12 in isolate IPO-94243 (Figure 2A). The accessory chromosomes were extremely variable as multiple chromosomes were either partially or completely absent (Figure 2A and B). Chromosomes 18 and 21 showed the highest absence frequency, $48 \%$ and $24 \%$, respectively, while partial deletions mainly affected chromosomes 14, 16 and 18 (52\%, 41\% and 36\%, respectively). On the other hand, chromosomes 17, 19 and 20 showed the least polymorphisms and were present in $86 \%$ of the isolates. We did not encounter an isolate without any accessory chromosome, but isolate IPO-93014 only carried chromosome 20 and isolate ND95 carried only chromosomes 16 and 20, which we validated by pulsed field gel electrophoresis (Figure $2 \mathrm{~A}$ and $\mathrm{C}$ ). Taken together, core chromosomes of $Z$. tritici isolates are always present but individual chromosomes may be duplicated, while the number of accessory chromosomes is highly variable with frequent total and partial deletions, although they are never completely absent (Figure 2).

The SNP density of the reference isolate Z. tritici IPO323 is higher in accessory chromosomes than in core chromosomes (Goodwin et al. 2011; Stukenbrock et al. 2011). Here, we assessed the SNP density in the entire panel by averaging the SNP counts over 1-kb stretches of core and accessory chromosomes over the genomewide SNP counts. The SNP densities in accessory chromosomes (average 1.29 SNPs per $\mathrm{kb})$ are higher than in the core chromosomes (average 1.01 SNPs per kb). This was particularly evident for chromosome 16, which showed a higher SNP density than the other chromosomes, while the lowest SNP density was observed in chromosome 14 (Figure S2). Thus, Z. tritici accessory chromosomes are much more polymorphic than core chromosomes with frequent large-scale structural variations and higher SNP rates.

Figure 2. Conserved core chromosomes and highly polymorphic accessory chromosomes in a global Zymoseptoria tritici panel. (A) Heatmap indicating the presence or absence of chromosomes in $136 \mathrm{Z}$. tritici isolates based on whole-genome sequencing data. Colors indicate whether a chromosome is present (black), partially duplicated (red), duplicated (red), partially deleted (green), or deleted (white). (B) Summary of chromosomal polymorphisms for all Z. tritici isolates. The frequencies of presence, partial duplication, duplication, partial deletion, and deletion are shown for each of the 21 chromosomes depicted as vertical bars. (C) Pulsed-field gel electrophoresis (PFGE) of ND95 and reference Z. tritici isolate IPO323. Chromosomal bands corresponds to indicated accessory chromosomes. 

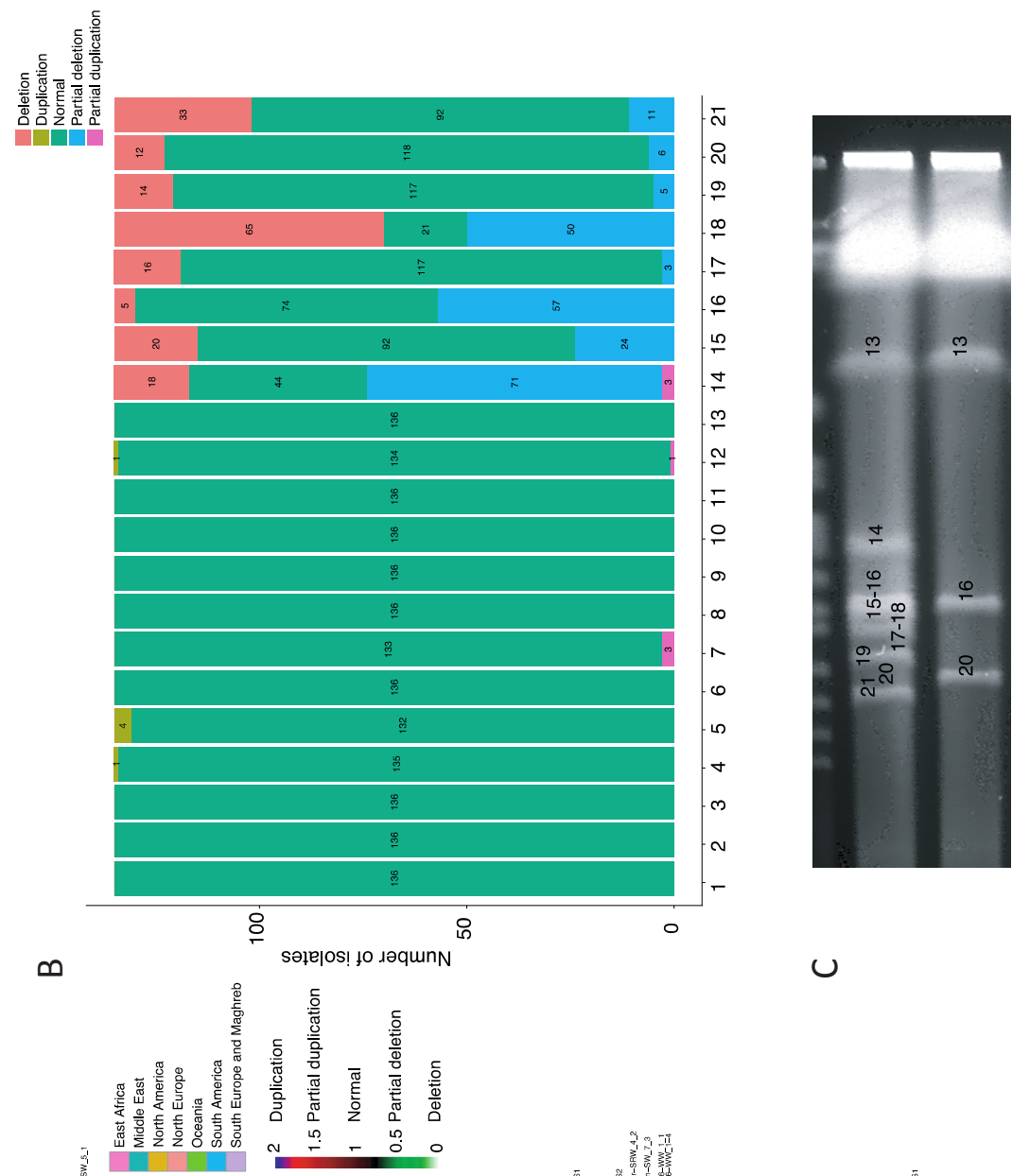

$\cup$

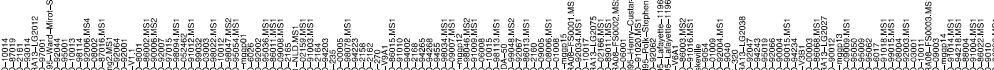

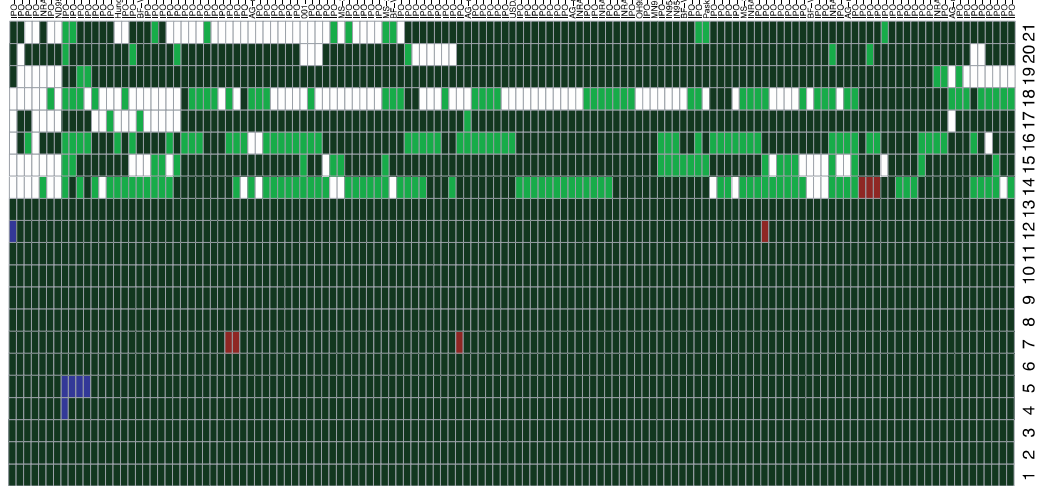

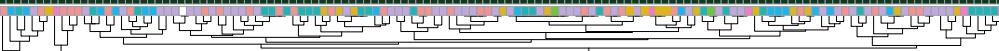

$\varangle$ 


\section{Diversified effector catalogues among $Z$. tritici isolates}

We predicted the effector repertoire of the reference isolate Z. tritici IPO323, and used it to assess effector diversity in the entire panel. We identified 1,034 genes that encode secreted proteins, of which 299 were predicted as effector candidates. The identified effector candidates, which also include the previously characterized effector AvrStb6 (Kema et al. 2018; Zhong et al. 2017), lacked conserved or functional domains (Table S3).

To identify presence/absence polymorphisms of effector genes in the entire panel, we mapped the genomic reads of all isolates to the reference strain IPO323 gene set and identified on average 12,250 genes per isolate, representing 93\% of the 13,157 genes determined in IPO323. Among these genes, approximately 270 effector genes were predicted for each isolate, representing $90 \%$ of the 299 effectors predicted by SignalP. Comparison of these data revealed that 183 (60\%) effectors are conserved in all $Z$. tritici isolates. Among the remaining presence/absence polymorphisms, we identified 88 effectors that were partially absent and 28 effectors that were only found in the reference isolate $Z$. tritici IPO323 (Figure S3). The majority $(285,95 \%)$ of these effectors was located on the core chromosomes and they were either conserved or showed presence/absence polymorphisms, whereas only 14 effectors were present on the accessory chromosomes and merely showed presence/absence polymorphisms (Figure S3). Of the known core effectors, we found that the previously characterized effector AvrStb6 was present in all isolates, except in isolate IPO-03003 (Figure 3).

To further investigate the differences between effector genes and other genes across the panel, we assessed gene length, distance to TEs, inter-genic length, and in planta expression levels. We observed that genes with presence/absence polymorphisms were significantly shorter than conserved genes (Wilcoxon rank-sum test, $P<0.05$ ) (Figure $4 \mathrm{~A}$ ). Effector genes are generally shorter than other genes, but we did not find differences between conserved effectors and those showing presence/absence polymorphisms (Figure 4A). We also determined that genes and effector genes showing presence/absence polymorphisms were significantly closer to TEs than conserved ones (Wilcoxon rank-sum test, $P<0.05$ ) (Figure 3B), but we did not observe an over-representation of effectors in gene-poor, TE-rich regions of $Z$. tritici genome (Figure $4 \mathrm{~A}$ ). Moreover, compared to conserved genes, the in planta expression of genes and effector genes with presence/ absence polymorphisms was significantly lower (Wilcoxon rank-sum test, $P<0.05$ ) (Figure $4 A)$. Finally, we assessed the relative diversity of candidate effector genes in the global panel and determined that other genes displayed more synonymous SNPs than effector genes and that these carry more non-synonymous SNPs that lead to amino acid changes than other genes (Figure 4B). For instance, the previously characterized effector gene AvrStb6 (Kema et al. 2018; Zhong et al. 2017) showed seven non-synonymous SNPs across the panel, leading to missense mutations and promoting virulence of $Z$. tritici isolates to the bread wheat cultivar Shafir (Kema et al. 2018). 


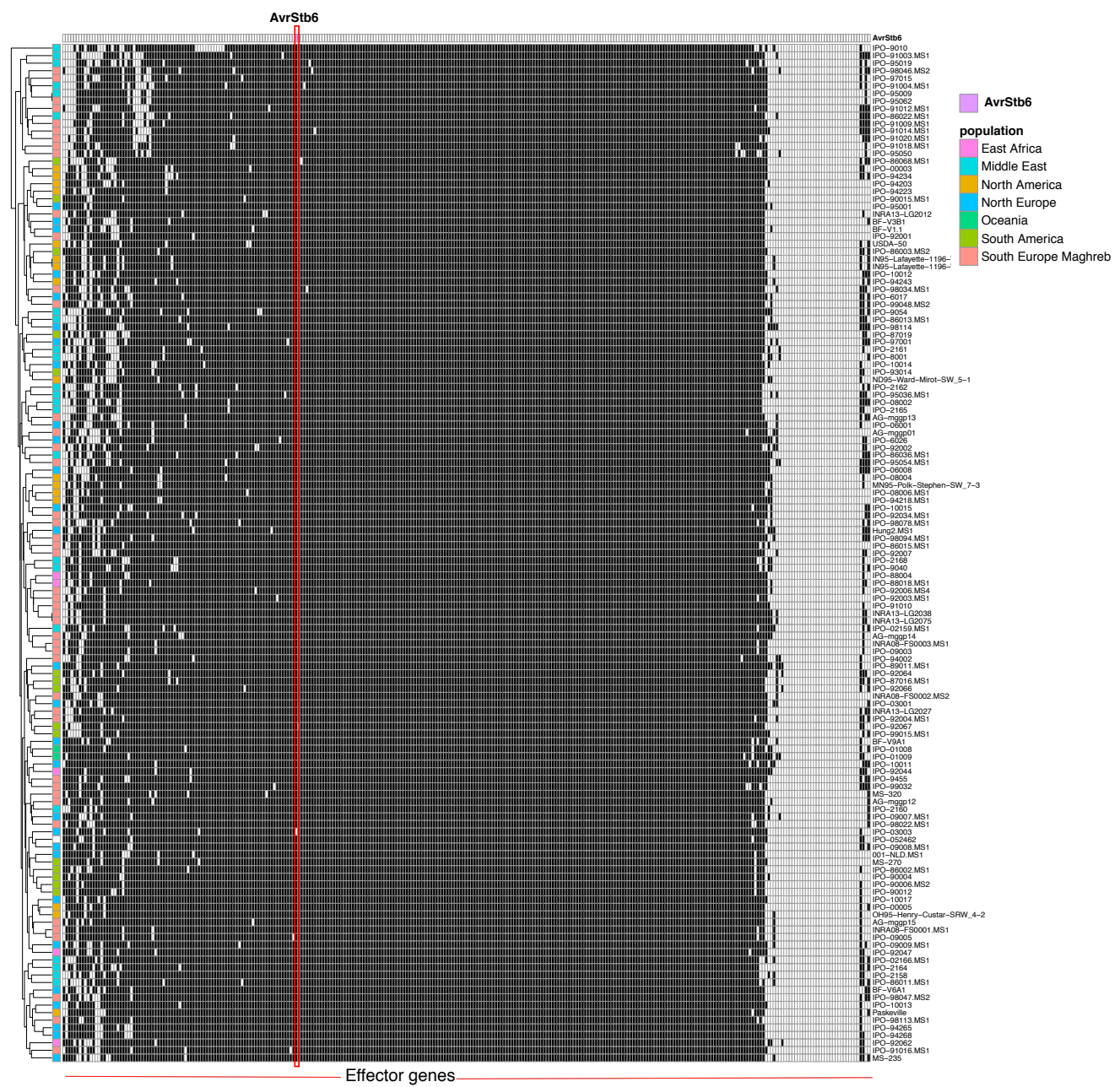

Figure 3. Heatmap indicating the presence or absence of effector genes in 136 Z. tritici isolates based on the gene annotation of reference $Z$. tritici isolate IPO323. The identified Z. tritici effector AvrStb6 is indicated and the red box indicates that the effector is absent in one isolates originating from Germany. 

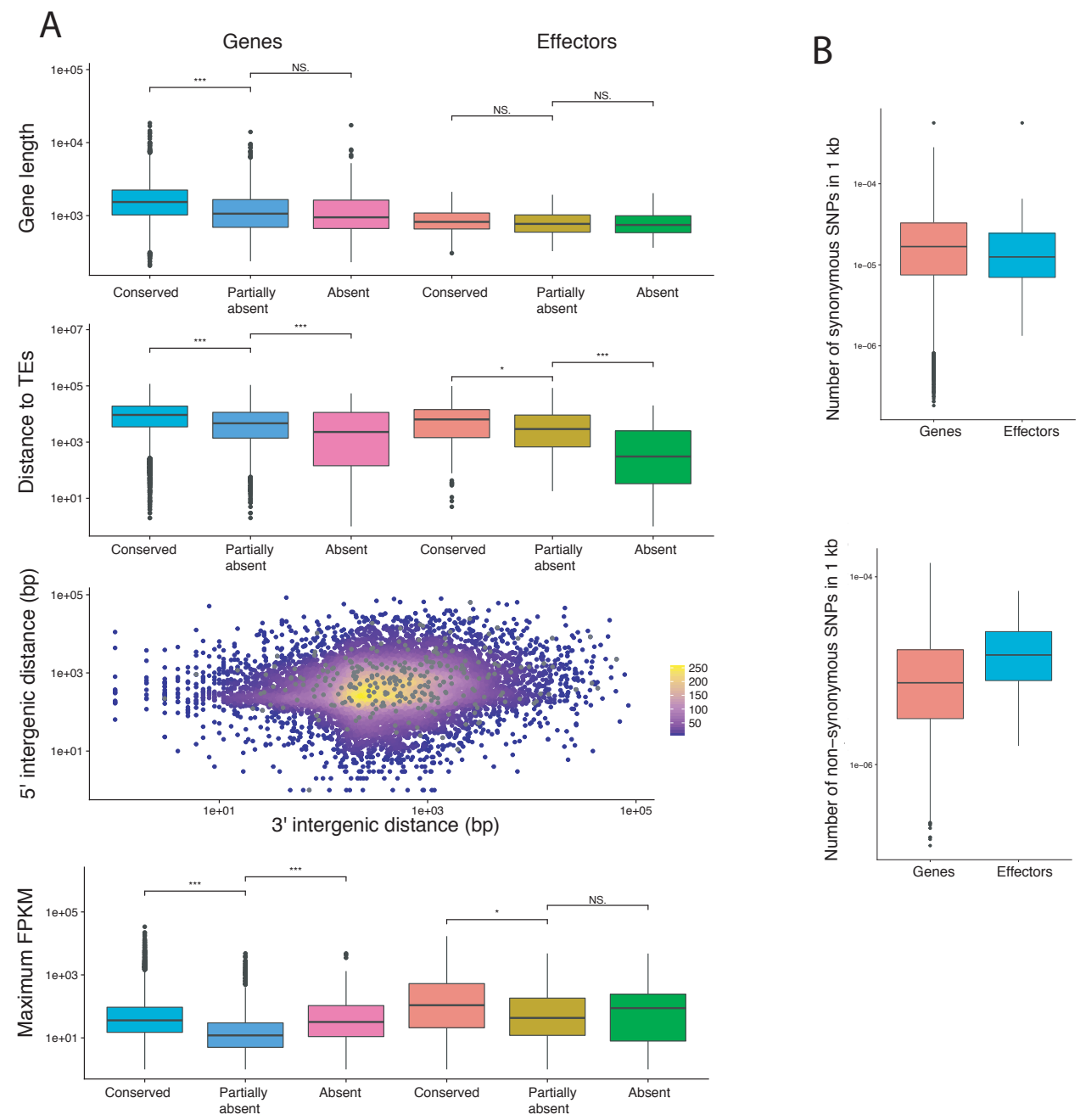

Figure 4. Effector diversification across a global Zymoseptoria tritici panel. (A) Features of conserved and polymorphic genes and effector genes in Z. tritici, including (first panel) gene length (kb), (second panel) distance to closest TEs, (third panel) inter-genic length of genes (blue) and effector genes (gray), (fourth panel) and in planta expression. (B) Boxplots showing the number of synonymous and non-synonymous SNPs for genes and effectors in one kb.

\section{Divergence of $\boldsymbol{Z}$. tritici pathogenicity to wheat species and cultivars}

In total, 118 isolates from the global panel (Table S1) were phenotyped on a defined set of host genotypes and we observed clear host specialization. Among the 83 Z. tritici isolates originating from BW, 61 isolates were only virulent on at least one of the three BW cultivars, while four out of the ten $Z$. tritici isolates originating from DW were virulent on 
the DW cv. Volcani 447 (Table S2). For example, DW isolate IPO95019 is non-pathogenic on all the BW cultivars (5\% pycnidia), while it is highly virulent on DW cv. Volcani 447 (40\% pycnidia) (Figure 5A). In contrast, BW isolate IPO02158 was non-pathogenic on DW cV. Volcani 447 (5\% pycnidia), but highly virulent on all three BW cultivars (30-40\% pycnidia) (Figure 5A).

We observed that Iranian isolates were the most aggressive on BW cvs. Taichung 29 (77\% pycnidia), Bulgaria 88 (66\% pycnidia), and TE9111 (57\% pycnidia) (Table S2). In general, the most agressive isolates on BW cvs. originated from the Middle East population with an average pycnidia percentage of $30 \%$ (Figure 5B), while the most agressive isolates on cv. Volcani 447 originated from South Europe and the Maghreb (Figure 5B). Thus, our study shows that the most virulent isolates on BW or DW cvs. originate from geographic regions associated with their origin or primary production area. Moreover, we identified 12 isolates with virulence to both BW and DW cultivars (Table S2).
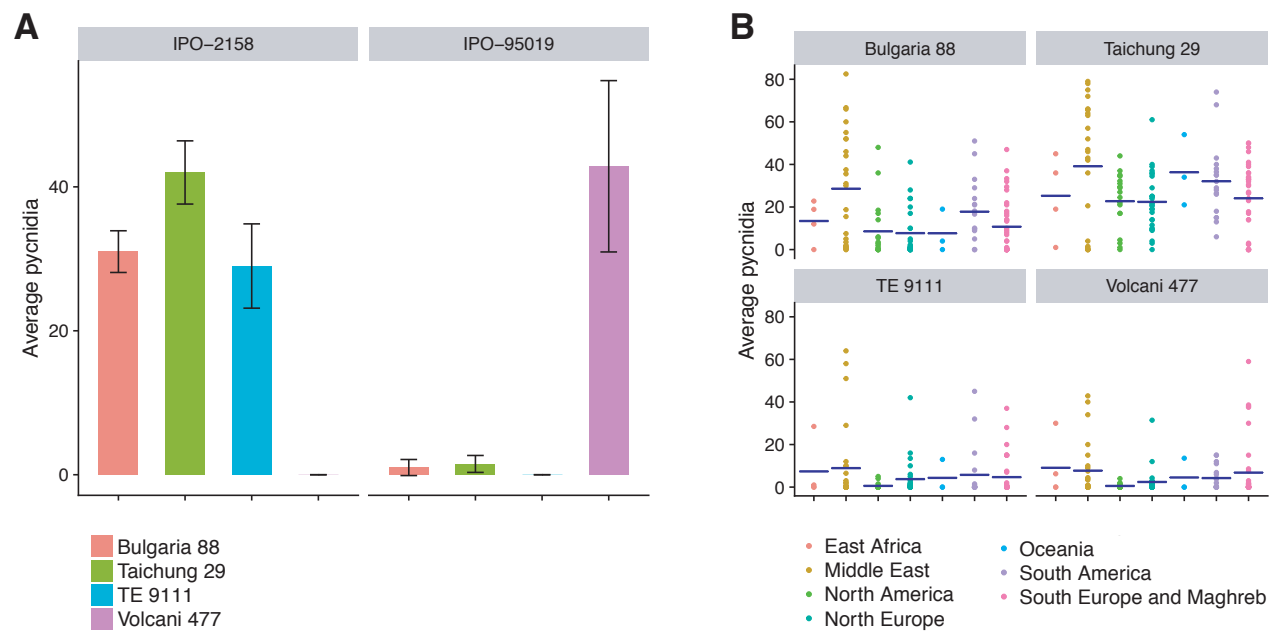

Figure 5. Divergence of wheat host and cultivar specificity in a global Zymoseptoria tritici panel. (A) Average pathogenicity of two exemplary Z. tritici isolates adapted to bread wheat (IPO-2158) or durum wheat (IPO-95019) on the bread wheat cvs. Bulgaria 88, Taichung 29, and TE9111, and the durum wheat cv. Volcani 477. Error bars represent standard error of two biological replicates. (B) Average pathogenicity of all Z. tritici isolates on the four phenotyped wheat cultivars. Blue horizontal lines represent average pycnidia levels for each population.

Genome-wide differences between bread and durum wheat adapted $Z$. tritici isolates To further explore the observed differences and adaptation to BW or DW, we assessed the genetic make-up of isolates with specificity to either BW or DW with respect to the presence of core and accessory chromosomes and effectors. As expected, core chromosomes are maintained amongst all isolates, but accessory chromosomes are 
highly variable (Figure S4A and B). We also determined that many candidate effectors are conserved between bread and durum isolates, including AvrStb6 (Figure S4C). Hence, it seems that pathogenicity differences between BW and DW isolates are associated with other polymorphisms in effector genes such as SNPs. Therefore, we assessed SNPS in the protein coding regions of all genes of the BW and DW isolates and found that isolates with BW specificity harbour 349 genes with SNPs leading to an amino acid change or a stop codon, but these included only four effector genes (Figure 6A and B).

A

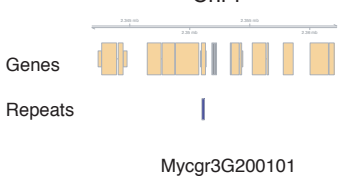

Mycgr3G200101

B

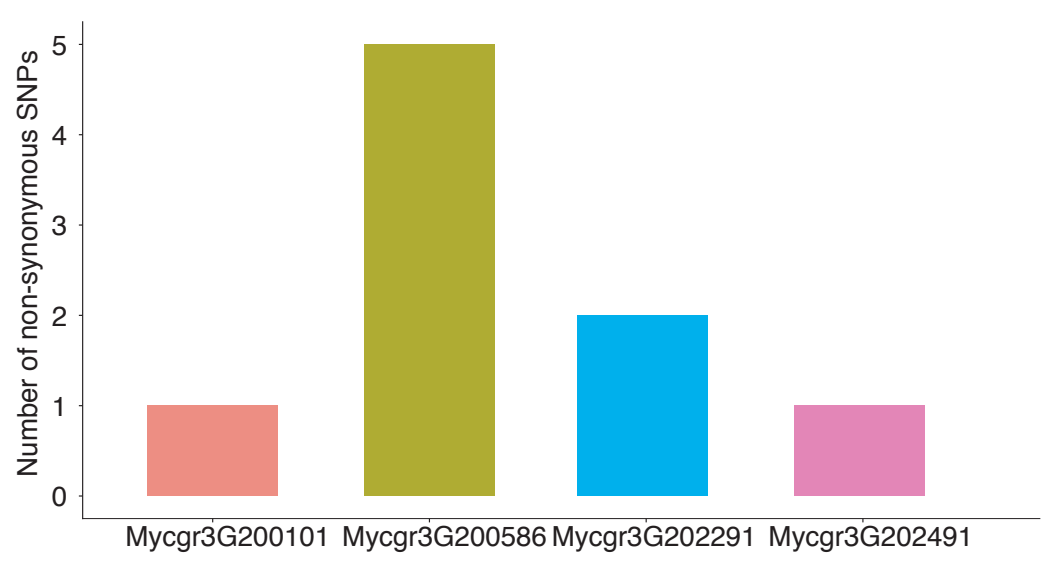

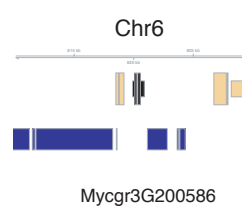

Mycgr3G200586

1

Mycgr3G202291

Chr11

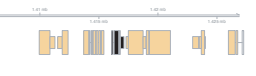

Mycgr3G202491

Chr12

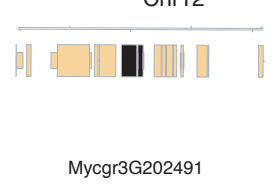

Figure 6. Genome-wide differences reveal candidate effector genes affected by SNPs in Zymoseptoria tritici isolates adapted to bread or durum wheat. (A) Genomic environment of identified effector candidates. Genes (yellow), and TEs (blue) are shown on chromosomes 4, 6, 11 and 12 in $10 \mathrm{~kb}$ regions upstream and downstream of each effector gene (black). (B) The identified number of non-synonymous SNPs in each effector gene. 


\section{DISCUSSION}

Global food security is one of the most important issues for humanity, which is threatened by plant pathogens that largely impact crop production (Fisher et al. 2018). To develop durable disease management strategies, knowledge on the evolutionary potential of pathogens in agricultural and natural environments is required (Plissonneau et al. 2017). Using population genomics, pathogen dynamics and genetic diversity can be assessed, thereby providing insights into pathogen adaptation to their hosts (Gibriel et al. 2016; Plissonneau et al. 2017; Stukenbrock and Bataillon 2012). For instance, population genomics of the ash dieback pathogen Hymenoscyphus fraxineus revealed the recent migration of this pathogen form East to West Europe and its extended genetic diversity in native Asian populations (McMullan et al. 2018). Similarly, population genomic analyses of $Z$. tritici revealed a rapidly evolving pathogen adapted to infect wheat, which emphasized the challenges imposed by this pathogen on future breeding programs (McDonald et al. 2016; Plissonneau et al. 2017; Plissonneau et al. 2018; Stukenbrock and Bataillon 2012; Stukenbrock et al. 2010). Recently, we provided new insights into the epidemiology of $Z$. tritici by showing its ability for sexual reproduction under adverse conditions that result in population sweeps for fungicide resistance and the slow down of the invasion of virulence alleles in natural populations (Chapter 6). Here, we performed comparative population genomics, using a global collection of $Z$. tritici isolates that were sampled from BW and DW accessions in the major global wheat producing regions. We demonstrated their high SNP rate, extensive structural variation on accessory chromosomes, and genome-wide differences between BW and DW isolates, which presumably contribute to the dynamic nature of $Z$. tritici populations (Croll et al. 2013; Hartmann et al. 2017; Linde et al. 2002; McDonald et al. 2016; Stukenbrock et al. 2011).

By phenotyping 118 isolates from the global panel on a defined set of BW and DW cultivars, we observed clear host specialization, which is consistent with previous studies showing that $Z$. tritici isolates are either virulent on BW or DW cultivars (Mirzadi Gohari et al. 2015). Thus, we aimed to assess the genomes of BW and DW isolates in order to identify causal presence/absence polymorphisms. We observed large scale structural variation in accessory chromosomes and conserved core chromosomes in BW and DW Z. tritici isolates, as expected for this fungal pathogen (Croll et al. 2013; Stuckenbrock et al. 2011). Next, we aimed to assess the differences in effector repertoires of BW and DW isolates, as modifications in effectors enable pathogens to adapt to their hosts (Seidl et al. 2014). One mechanism to diversify effectors is through the occurrence of SNPs. Once in protein coding regions of effector genes these might be shared among virulent isolates but should be absent in avirulent ones. Thus, we reasoned that conserved effectors of $Z$. tritici, albeit being present in virulent and avirulent isolates, facilitate adaptation to infect particular wheat cultivars. By assessing genome-wide differences between BW and DW isolates, we identified four effector genes with non-synonymous SNPs only leading to a different amino acid or a stop codon in bread wheat isolates. 
The previously identified conserved effectors AvrStb6 and Avr3D1 carry SNPs shared by virulent isolates allowing them to circumvent wheat immunity (Kema et al. 2018; Meile et al. 2018a; Zhong et al. 2017). Future functional analyses are needed to investigate the role of the identified effectors and SNPs in Z. tritici host specificity. Additionally, since the $Z$. tritici reference strain IPO323 is a bread wheat isolate, mapping genomic reads of DW isolates to this reference genome will not disclose DW lineage-specific regions. Therefore, a reference $\mathrm{DW}$ isolate genome is required to further assess the difference between BW and DW isolates, as gene absence also has considerable impact on pathogen virulence (Fouché et al. 2018a). For example, aggressiveness of Z. tritici isolate 3D7 towards wheat cv. Tornonit is associated with deletion of the effector gene Zt_6_809 (Hartmann et al. 2017). Similarly, in V. dahliae, deletion of the Ave1 effector gene is associated with virulence on tomato plants that carry the Ve1 immune receptor (de Jonge et al. 2012).

A truly comprehensive global overview of genetic diversity of Z. tritici is unavailable, as many wheat-growing regions throughout are significantly under-represented due to lack of sampling. For example, little is known about the $Z$. tritici genetic diversity in Asia, Africa, as well as South America, despite their importance for global wheat production (Cordo et al. 2017). We demonstrated the genome-wide diversity by comparing chromosome and effector diversity of $136 \mathrm{Z}$. tritici isolates that were sampled from many and diverse wheat-growing regions. First, we identified a high number of SNPs in these $Z$. tritici populations, indicating a high level of genetic diversity, comparable with previous population studies (Hartmann et al. 2017; McDonald et al. 2016). Of all populations, the Middle East population displayed the highest number of SNPs (average number of 440,977 SNPs per isolate), highlighting the huge genetic diversity among Z. tritici isolates originating from the centre of origin (Stukenbrock et al. 2006; Stukenbrock et al. 2011), which likely explains why these isolates are pathogenic across the phenotyped BW and DW cultivars. Subsequent clustering of the isolates based on the identified SNPs showed highly polymorphic populations that did not align with their geographical origin or hosts. This is in accordance with previous studies that showed no or little population structure of global $Z$. tritici isolates at local, regional, and worldwide scales (Linde et al. 2002; Zhan et al. 2003), indicating extensive genetic diversity between Z. tritici populations. Similarly, we observed mixed Z. tritici populations when we performed the structure analysis, likely suggesting high rates of gene flow (Linde et al. 2002; Zhan et al. 2003). In contrast, recent population genomics studies displayed genetic relatedness between pathogen populations of the same origin (El Chartouni et al. 2011; Hartmann et al. 2017). When we assessed the diversity of Z. tritici chromosomes in our global collection, we observed, in line with previous work, highly conserved core chromosomes with extensive size polymorphisms and variable numbers and sizes of accessory chromosomes (Croll et al. 2013; Habig et al. 2017; Wittenberg et al. 2009). Z. tritici accessory chromosomes can also be lost during asexual propagation (Moeller et al. 2018), and their loss might be linked to increased aggressiveness on wheat as suggested for the cvs. Titlis and Runal (Habig et al. 2017; Stewart et al. 2018). 
Lastly, we assessed the polymorphism of effector genes and observed high numbers of conserved effectors affected by non-synonymous SNPs, highlighting the importance of such effectors in Z. tritici virulence. This is in contrast to other fungal pathogens, such as Fusarium oxysporum and Alternaria alternata, where effector genes or host-specific toxin genes are mainly located on accessory chromosomes (Ma et al. 2010; Thomma 2003). Thus, our study highlights the significant genetic diversity in global populations of Z. tritici and signifies the importance of conserved effectors in the evolution of virulence on host plants.

In conclusion, our data demonstrate the extensive variability in a global population of Z. tritici isolates. We have demonstrated that the largest genetic diversity was observed in isolates originating from the centre of origin of the pathogen and the host, and observed four effector genes affected by non-synonymous SNPs that might contribute to host specificity. The extensive genetic diversity among $Z$. tritici isolates highlights its evolutionary potential to infect and overcome resistance in new wheat varieties. This challenges the overall question of durable management of this important wheat disease.

\section{ACKNOWLEDGEMENTS}

MFS acknowledges the receipt of a VENI grant of ALW-NWO. 


\section{REFERENCES}

Adhikari, T. B., Yang, X., Cavaletto, J. R., Hu, X., Buechley, G., Ohm, H. W., Shaner, G., and Goodwin, S. B. 2004. Molecular mapping of Stb1, a potentially durable gene for resistance to septoria tritici blotch in wheat. Theoretical and Applied Genetics 109:944-953.

Bertels, F., Silander, O. K., Pachkov, M., Rainey, P. B., and van Nimwegen, E. 2014. Automated reconstruction of whole-genome phylogenies from short-sequence reads. Molecular Biology and Evolution 31:1077-1088.

Brown, J. K. M., Chartrain, L., Lasserre-Zuber, P., and Saintenac, C. 2015. Genetics of resistance to Zymoseptoria tritici and applications to wheat breeding. Fungal Genetics and Biology 79:33-41.

Brunner, P. C., and McDonald, B. A. 2018. Evolutionary analyses of the avirulence effector AvrStb6 in global populations of Zymoseptoria tritici identify candidate amino acids involved in recognition. Molecular Plant Pathology. https://doi.org/10.1111/ mpp.12662E-pub ahead of print.

Chartrain, L., Brading, P. A., and Brown, J. K. M. 2005a. Presence of the Stb6 gene for resistance to septoria tritici blotch (Mycosphaerella graminicola) in cultivars used in wheat-breeding programmes worldwide. Plant Pathology 54:134-143.

Chartrain, L., Joaquim, P., Berry, S. T., Arraiano, L. S., Azanza, F., and Brown, J. K. 2005b. Genetics of resistance to septoria tritici blotch in the Portuguese wheat breeding line TE 9111. Theoretical and Applied Genetics 110:1138-1144.

Cingolani, P., Platts, A., Wang le, L., Coon, M., Nguyen, T., Wang, L., Land, S. J., Lu, X., and Ruden, D. M. 2012. A program for annotating and predicting the effects of single nucleotide polymorphisms, SnpEff: SNPs in the genome of Drosophila melanogaster strain w1118; iso-2; iso-3. Fly 6:80-92.

Cook, D. E., Mesarich, C. H., and Thomma, B. P. H. J. 2015. Understanding plant immunity as a surveillance system to detect invasion. Annual Review of Phytopathology 53:541-563.

Cordo, C. A., Mónaco, C. I., Altamirano, R., Perelló, A., Larrán, S., Kripelz, N. I., and Simón, M. 2017. Weather conditions associated with the release and dispersal of Zymoseptoria tritici spores in the Argentine Pampas region. International Journal of Agronomy 2017.

Croll, D., Lendenmann, M. H., Stewart, E., and McDonald, B. A. 2015. The impact of recombination hotspots on genome evolution of a fungal plant pathogen. Genetics 201: 1213-1228.

Croll, D., Zala, M., and McDonald, B. A. 2013. Breakage-fusion-bridge cycles and large insertions contribute to the rapid evolution of accessory chromosomes in a fungal pathogen. PLoS Genetics 9:e1003567.

Danecek, P., Auton, A., Abecasis, G., Albers, C. A., Banks, E., DePristo, M. A., Handsaker, R. E., Lunter, G., Marth, G. T., Sherry, S. T., McVean, G. 2011. The variant call format and VCFtools. Bioinformatics 27:2156-2158. 
de Jonge, R., Bolton, M. D., Kombrink, A., van den Berg, G. C., Yadeta, K. A., and Thomma, B. P. 2013. Extensive chromosomal reshuffling drives evolution of virulence in an asexual pathogen. Genome Research 23:1271-1282.

de Jonge, R., Peter van Esse, H., Maruthachalam, K., Bolton, M. D., Santhanam, P., Saber, M. K., Zhang, Z., Usami, T., Lievens, B., Subbarao, K. V., and Thomma, B. P. H. J. 2012. Tomato immune receptor Ve1 recognizes effector of multiple fungal pathogens uncovered by genome and RNA sequencing. Proceedings of the National Academy of Sciences 109:5110-5115.

de Jonge, R., and Thomma, B. P. H. J. 2009. Fungal LysM effectors: extinguishers of host immunity? Trends in Microbiology 17:151-157.

Dobin, A., Davis, C. A., Schlesinger, F., Drenkow, J., Zaleski, C., Jha, S., Batut, P., Chaisson, M., and Gingeras, T. R. 2013. STAR: ultrafast universal RNA-seq aligner. Bioinformatics 29:15-21.

Dong, S., Raffaele, S., and Kamoun, S. 2015. The two-speed genomes of filamentous pathogens: waltz with plants. Current Opinion in Genetics \& Development 35:5765.

El Chartouni, L., Tisserant, B., Siah, A., Duyme, F., Leducq, J.-B., Deweer, C., Fichter-Roisin, C., Sanssené, J., Durand, R., Halama, P., and Reignault, P. 2011. Genetic diversity and population structure in French populations of Mycosphaerella graminicola. Mycologia 103:764-774.

Faino, L., Seidl, M. F., Shi-Kunne, X., Pauper, M., van den Berg, G. C. M., Wittenberg, A. H. J., and Thomma, B. P. H. J. 2016. Transposons passively and actively contribute to evolution of the two-speed genome of a fungal pathogen. Genome Research 26:1091-1100.

Falush, D., Stephens, M., and Pritchard, J. K. 2003. Inference of population structure using multilocus genotype data: linked loci and correlated allele frequencies. Genetics 164:1567-1587.

FAOSTAT, F. 2015. FAO Statistics Division; 2014.

Fisher, M. C., Hawkins, N. J., Sanglard, D., and Gurr, S. J. 2018. Worldwide emergence of resistance to antifungal drugs challenges human health and food security. Science 360:739-742.

Fisher, M. C., Henk, D. A., Briggs, C. J., Brownstein, J. S., Madoff, L. C., McCraw, S. L., and Gurr, S. J. 2012. Emerging fungal threats to animal, plant and ecosystem health. Nature 484:186-194.

Flutre, T., Inizan, O., Hoede, C., and Quesneville, H. 2010. REPET: pipelines for the identification and annotation of transposable elements in genomic sequences. Pages 9-13 in: Proceedings of the Plant \& Animal Genome (PAG) XVIII Conference, San Diego, CA, USA.

Fouché, S., Plissonneau, C., and Croll, D. 2018a. The birth and death of effectors in rapidly evolving filamentous pathogen genomes. Current Opinion in Microbiology. 46:34-42. 
Fouché, S., Plissonneau, C., McDonald, B. A., and Croll, D. 2018b. Meiosis leads to pervasive copy-number variation and distorted inheritance of accessory chromosomes of the wheat pathogen Zymoseptoria tritici. Genome Biology Evolution 10:1416-1429.

Gibriel, H. A. Y., Thomma, B. P. H. J., and Seidl, M. F. 2016. The age of effectors: genomebased discovery and applications. Phytopathology 106: 1206-1212.

Goodwin, S. B., M'Barek, S. B., Dhillon, B., Wittenberg, A. H., Crane, C. F., Hane, J. K., Foster, A. J., Lee, T. A., Grimwood, J., and Aerts, A. 2011. Finished genome of the fungal wheat pathogen Mycosphaerella graminicola reveals dispensome structure, chromosome plasticity, and stealth pathogenesis. PLoS Genetics 7:e1002070.

Grandaubert, J., Dutheil, J. Y., and Stukenbrock, E. H. 2018. The genomic determinants of adaptive evolution in a fungal pathogen. bioRxiv: 176727.

Grünwald, N. J., McDonald, B. A., and Milgroom, M. G. 2016. Population genomics of fungal and oomycete pathogens. Annual Review of Phytopathology 54:323-346.

Haas, B. J., Kamoun, S., Zody, M. C., Jiang, R. H. Y., Handsaker, R. E., Cano, L. M., Grabherr, M., Kodira, C. D., Raffaele, S., Torto-Alalibo, T., Bozkurt, T. O., Ah-Fong, A. M. V., Alvarado, L., Anderson, V. L., Armstrong, M. R., Avrova, A., Baxter, L., Beynon, J., Boevink, P. C., Bollmann, S. R., Bos, J. I. B., Bulone, V., Cai, G., Cakir, C., Carrington, J. C., Chawner, M., Conti, L., Costanzo, S., Ewan, R., Fahlgren, N., Fischbach, M. A., Fugelstad, J., Gilroy, E. M., Gnerre, S., Green, P. J., Grenville-Briggs, L. J., Griffith, J., Grünwald, N. J., Horn, K., Horner, N. R., Hu, C.-H., Huitema, E., Jeong, D.-H., Jones, A. M. E., Jones, J. D. G., Jones, R. W., Karlsson, E. K., Kunjeti, S. G., Lamour, K., Liu, Z., Ma, L., Maclean, D., Chibucos, M. C., McDonald, H., McWalters, J., Meijer, H. J. G., Morgan, W., Morris, P. F., Munro, C. A., O’Neill, K., Ospina-Giraldo, M., Pinzón, A., Pritchard, L., Ramsahoye, B., Ren, Q., Restrepo, S., Roy, S., Sadanandom, A., Savidor, A., Schornack, S., Schwartz, D. C., Schumann, U. D., Schwessinger, B., Seyer, L., Sharpe, T., Silvar, C., Song, J., Studholme, D. J., Sykes, S., Thines, M., van de Vondervoort, P. J. I., Phuntumart, V., Wawra, S., Weide, R., Win, J., Young, C., Zhou, S., Fry, W., Meyers, B. C., van West, P., Ristaino, J., Govers, F., Birch, P. R. J., Whisson, S. C., Judelson, H. S., and Nusbaum, C. 2009. Genome sequence and analysis of the Irish potato famine pathogen Phytophthora infestans. Nature 461:393-398.

Habig, M., Quade, J., and Stukenbrock, E. H. 2017. Forward genetics approach reveals host genotype-dependent importance of accessory chromosomes in the fungal wheat pathogen Zymoseptoria tritici. mBio 8:e01919-17.

Hartmann, F. E., McDonald, B. A., and Croll, D. 2018. Genome-wide evidence for divergent selection between populations of a major agricultural pathogen. Molecular Ecology 9:656-617.

Hartmann, F. E., Sanchez-Vallet, A., McDonald, B. A., and Croll, D. 2017. A fungal wheat pathogen evolved host specialization by extensive chromosomal rearrangements. ISME Journal 11:1189-1204.

Hubbard, A., Lewis, C., Yoshida, K., Ramirez-Gonzalez, R., de Vallavieille-Pope, C., Thomas, J., Kamoun, S., Bayles, R., Uauy, C., and Saunders, D. 2015. Field pathogenomics reveals the emergence of a diverse wheat yellow rust population. Genome Biology $16: 23$. 
Kema, G., Annone, J., Sayoud, R., and Van Silfhout, C. 1996. Genetic variation for virulence and resistance in the wheat-Mycosphaerella graminicola pathosystem. I. Interactions between pathogen isolates and host cultivars. Phytopathology 86:200212.

Kema, G. H. J., Mirzadi Gohari, A., Aouini, L., Gibriel, H. A. Y., Ware, S. B., van den Bosch, F., Manning-Smith, R., Alonso-Chavez, V., Helps, J., Ben M'Barek, S., Mehrabi, R., Diaz-Trujillo, C., Zamani, E., Schouten, H. J., van der Lee, T. A. J., Waalwijk, C., de Waard, M. A., De Wit, P. J. G. M., Verstappen, E. C. P., Thomma, B. P. H. J., Meijer, H. J. G., and Seidl, M. F. 2018. Stress and sexual reproduction affect the dynamics of the wheat pathogen effector AvrStb6 and strobilurin resistance. Nature Genetics 23:678.

Kettles, G. J., and Kanyuka, K. 2016. Dissecting the molecular interactions between wheat and the fungal pathogen Zymoseptoria tritici. Frontiers in Plant Science 7:508.

Kolde, R., and Kolde, M. R. 2015. Package 'pheatmap'.

Kombrink, A., Rovenich, H., Shi-Kunne, X., Rojas-Padilla, E., van den Berg, G. C. M., Domazakis, E., de Jonge, R., Valkenburg, D.-J., Sánchez-Vallet, A., Seidl, M. F., and Thomma, B. P. H. J. 2016. Verticillium dahliae LysM effectors differentially contribute to virulence on plant hosts. Molecular Plant Pathology 18: 596-608.

Kombrink, A., and Thomma, B. P. H. J. 2013. LysM Effectors: Secreted Proteins Supporting Fungal Life. PLoS Pathogens 9:e1003769.

Langmead, B., and Salzberg, S. L. 2012. Fast gapped-read alignment with Bowtie 2. Nature Methods 9:357-359.

Li, H., and Durbin, R. 2010. Fast and accurate long-read alignment with Burrows-Wheeler transform. Bioinformatics 26:589-595.

Linde, C. C., Zhan, J., and McDonald, B. A. 2002. Population structure of Mycosphaerella graminicola: from lesions to continents. Phytopathology 92:946-955.

Ma, L. J., Does, H. C., Borkovich, K. A., Coleman, J. J., Daboussi, M. J., Pietro, A., Dufresne, M., Freitag, M., Grabherr, M., and Henrissat, B. 2010. Comparative genomics reveals mobile pathogenicity chromosomes in Fusarium. Nature 464:367-373.

Marshall, R., Kombrink, A., Motteram, J., Loza-Reyes, E., Lucas, J., Hammond-Kosack, K. E., Thomma, B. P. H. J., and Rudd, J. J. 2011. Analysis of two in planta expressed LysM effector homologs from the fungus Mycosphaerella graminicola reveals novel functional properties and varying contributions to virulence on wheat. Plant Physiology 156:756-769.

McDonald, M. C., McDonald, B. A., and Solomon, P. S. 2015. Recent advances in the Zymoseptoria tritici-wheat interaction: insights from pathogenomics. Frontiers in Plant Science 6:102.

McDonald, M. C., McGinness, L., Hane, J. K., Williams, A. H., Milgate, A., and Solomon, P. S. 2016. Utilizing gene tree variation to identify candidate effector genes in Zymoseptoria tritici. G3 6:779-791. 
McKenna, A., Hanna, M., Banks, E., Sivachenko, A., Cibulskis, K., Kernytsky, A., Garimella, K., Altshuler, D., Gabriel, S., Daly, M., and DePristo, M. A. 2010. The genome analysis toolkit: A MapReduce framework for analyzing next-generation DNA sequencing data. Genome Research 20:1297-1303.

McMullan, M., Rafiqi, M., Kaithakottil, G., Clavijo, B. J., Bilham, L., Orton, E., PercivalAlwyn, L., Ward, B. J., Edwards, A., Saunders, D. G. O., Garcia Accinelli, G., Wright, J., Verweij, W., Koutsovoulos, G., Yoshida, K., Hosoya, T., Williamson, L., Jennings, P., loos, R., Husson, C., Hietala, A. M., Vivian-Smith, A., Solheim, H., MaClean, D., Fosker, C., Hall, N., Brown, J. K. M., Swarbreck, D., Blaxter, M., Downie, J. A., and Clark, M. D. 2018. The ash dieback invasion of Europe was founded by two genetically divergent individuals. Nature Ecology Evolution 381:235.

Mehrabi, R., Taga, M., and Kema, G. H. 2007. Electrophoretic and cytological karyotyping of the foliar wheat pathogen Mycosphaerella graminicola reveals many chromosomes with a large size range. Mycologia 99:868-876.

Meile, L., Croll, D., Brunner, P. C., Plissonneau, C., Hartmann, F. E., McDonald, B. A., and Sánchez-Vallet, A. 2018a. A fungal avirulence factor encoded in a highly plastic genomic region triggers partial resistance to septoria tritici blotch. bioRxiv:264226.

Meile, L., Croll, D., Brunner, P. C., Plissonneau, C., Hartmann, F. E., McDonald, B. A., and Sánchez-Vallet, A. 2018b. A fungal avirulence factor encoded in a highly plastic genomic region triggers partial resistance to septoria tritici blotch. New Phytologist 65:512.

Mirzadi Gohari, A., Ware, S. B., Wittenberg, A. H. J., Mehrabi, R., Ben M'Barek, S., Verstappen, E. C. P., van der Lee, T. A. J., Robert, O., Schouten, H. J., de Wit, P. P. J. G. M., and Kema, G. H. J. 2015. Effector discovery in the fungal wheat pathogen Zymoseptoria tritici. Molecular Plant Pathology 16:931-945.

Moeller, M., Habig, M., Freitag, M., and Holtgrewe Stukenbrock, E. 2018. Extraordinary genome instability and widespread chromosome rearrangements during vegetative growth. bioRxiv:304915.

Möller, M., and Stukenbrock, E. H. 2017. Evolution and genome architecture in fungal plant pathogens. Nature Publishing Group 484:1-16.

Naouari, M., Siah, A., Elgazzah, M., Reignault, P., and Halama, P. 2016. Mitochondrial DNA-based genetic diversity and population structure of Zymoseptoria tritici in Tunisia. European Journal of Plant Pathology 146:305-314.

O'Driscoll, A., Kildea, S., Doohan, F., Spink, J., and Mullins, E. 2014. The wheat-Septoria conflict: a new front opening up? Trends in Plant Science 19:602-610.

Petersen TN, Brunak S, von Heijne G, Nielsen H (2011) SignalP 4.0: discriminating signal peptides from transmembrane regions. Nature Methods 8: 785-786.

Plissonneau, C., Benevenuto, J., Mohd-Assaad, N., Fouché, S., Hartmann, F. E., and Croll, D. 2017. Using population and comparative genomics to understand the genetic basis of effector-driven fungal pathogen evolution. Frontiers in Plant Sciences 8:119. 
Plissonneau, C., Hartmann, F. E., and Croll, D. 2018. Pangenome analyses of the wheat pathogen Zymoseptoria tritici reveal the structural basis of a highly plastic eukaryotic genome. BMC Biology 16:673.

Plissonneau, C., Stürchler, A., and Croll, D. 2016. The evolution of orphan regions in genomes of a fungal pathogen of wheat. mBio 7: e01231-16.

Poppe, S., Dorsheimer, L., Happel, P., and Stukenbrock, E. H. 2015. Rapidly evolving genes are key players in host specialization and virulence of the fungal wheat pathogen Zymoseptoria tritici (Mycosphaerella graminicola). PLoS Pathogens 11:e1005055.

Pritchard, J. K., Stephens, M., and Donnelly, P. 2000. Inference of population structure using multilocus genotype data. Genetics 155:945-959.

Quinlan, A. R., and Hall, I. M. 2010. BEDTools: a flexible suite of utilities for comparing genomic features. Bioinformatics 26: 841-842.

Raffaele, S., Farrer, R. A., Cano, L. M., Studholme, D. J., MacLean, D., Thines, M., Jiang, R. H., Zody, M. C., Kunjeti, S. G., Donofrio, N. M., Meyers, B. C., Nusbaum, C., and Kamoun, S. 2010. Genome evolution following host jumps in the Irish potato famine pathogen lineage. Science 330:1540-1543.

Raffaele, S., and Kamoun, S. 2012. Genome evolution in filamentous plant pathogens: why bigger can be better. Nature Reviews Microbiology 10:417-430.

Rovenich, H., Boshoven, J. C., and Thomma, B. P. H. J. 2014. Filamentous pathogen effector functions: of pathogens, hosts and microbiomes. Current Opinion in Plant Biology 20:96-103.

Rudd, J. J., Kanyuka, K., Hassani-Pak, K., Derbyshire, M., Andongabo, A., Devonshire, J., Lysenko, A., Saqi, M., Desai, N. M., Powers, S. J., Hooper, J., Ambroso, L., Bharti, A., Farmer, A., Hammond-Kosack, K. E., Dietrich, R. A., and Courbot, M. 2015. Transcriptome and metabolite profiling of the infection cycle of Zymoseptoria tritici on wheat reveals a biphasic interaction with plant immunity involving differential pathogen chromosomal contributions and a variation on the hemibiotrophic lifestyle definition. Plant Physiology 167:1158-1185.

Saintenac, C., Lee, W.-S., Cambon, F., Rudd, J. J., King, R. C., Marande, W., Powers, S. J., Bergès, H., Phillips, A. L., Uauy, C., Hammond-Kosack, K. E., Langin, T., and Kanyuka, K. 2018. Wheat receptor-kinase-like protein Stb6 controls gene-for-gene resistance to fungal pathogen Zymoseptoria tritici. Nature Genetics 51:291.

Sambrook, J., Fritsch, E. F., and Maniatis, T. 1989. Molecular cloning: a laboratory manual. Cold spring harbor laboratory press.

Seidl, M. F., and Thomma, B. 2017a. Transposable elements direct the coevolution between plants and microbes. Trends in Genetics 33:842-851.

Singh, R. P., Hodson, D. P., Huerta-Espino, J., Jin, Y., Bhavani, S., Njau, P., Herrera-Foessel, S., Singh, P. K., Singh, S., and Govindan, V. 2011. The emergence of Ug99 races of the stem rust fungus is a threat to world wheat production. Annual Review of Phytopathology 49:465-481.

Smit, A., Hubley, R., and Green, P. 2016. RepeatMasker Open-4.0. Available from: http:// www.repeatmasker.org 
Sperschneider, J., Gardiner, D. M., Dodds, P. N., Tini, F., Covarelli, L., Singh, K. B., Manners, J. M., and Taylor, J. M. 2015b. EffectorP: predicting fungal effector proteins from secretomes using machine learning. New Phytologist 210:743-761.

Stewart, E. L., Croll, D., Lendenmann, M. H., Sánchez-Vallet, A., Hartmann, F. E., PalmaGuerrero, J., Ma, X., and McDonald, B. A. 2018. Quantitative trait locus mapping reveals complex genetic architecture of quantitative virulence in the wheat pathogen Zymoseptoria tritici. Molecular Plant Pathology 19:201-216.

Stukenbrock, E. H., Banke, S., Javan-Nikkhah, M., and McDonald, B. A. 2006. Origin and domestication of the fungal wheat pathogen Mycosphaerella graminicola via sympatric speciation. Molecular Biology and Evolution 24:398-411.

Stukenbrock, E. H., and Bataillon, T. 2012. A population genomics perspective on the emergence and adaptation of new plant pathogens in agro-ecosystems. PLoS Pathogens 8:e1002893.

Stukenbrock, E. H., Bataillon, T., Dutheil, J. Y., Hansen, T. T., Li, R., Zala, M., McDonald, B. A., Wang, J., and Schierup, M. H. 2011. The making of a new pathogen: insights from comparative population genomics of the domesticated wheat pathogen Mycosphaerella graminicola and its wild sister species. Genome Research 21:21572166.

Stukenbrock, E. H., Jørgensen, F. G., Zala, M., Hansen, T. T., McDonald, B. A., and Schierup, M. H. 2010. Whole-genome and chromosome evolution associated with host adaptation and speciation of the wheat pathogen Mycosphaerella graminicola. PLoS Genetics 6:e1001189.

Thomma, B. P. H. J. 2003. Alternaria spp.: from general saprophyte to specific parasite. Molecular Plant Pathology 4:225-236.

Trapnell, C., Williams, B. A., Pertea, G., Mortazavi, A., Kwan, G., van Baren, M. J., Salzberg, S. L., Wold, B. J., and Pachter, L. 2010. Transcript assembly and quantification by RNA-Seq reveals unannotated transcripts and isoform switching during cell differentiation. Nature Biotechnology 28: 511-515.

Troccoli, A., Borrelli, G. M., De Vita, P., Fares, C., and Di Fonzo, N. 2000. Mini Review: Durum wheat quality: A multidisciplinary concept. Journal of Cereal Science 32:99113.

Wickham, H. 2015. ggplot2: elegant graphics for data analysis.[Internet]. 2009. New York, USA 907.

Wittenberg, A. H. J., van der Lee, T. A. J., Ben M'Barek, S., Ware, S. B., Goodwin, S. B., Kilian, A., Visser, R. G. F., Kema, G. H. J., and Schouten, H. J. 2009. Meiosis drives extraordinary genome plasticity in the haploid fungal plant pathogen Mycosphaerella graminicola. PLoS One 4:e5863.

Zhan, J., Pettway, R. E., and McDonald, B. A. 2003. The global genetic structure of the wheat pathogen Mycosphaerella graminicola is characterized by high nuclear diversity, low mitochondrial diversity, regular recombination, and gene flow. Fungal Genetics and Biology: 38:286-297. 
Zheng, X., Levine, D., Shen, J., Gogarten, S. M., Laurie, C., and Weir, B. S. 2012. A highperformance computing toolset for relatedness and principal component analysis of SNP data. Bioinformatics 28:3326-3328.

Zhong, Z., Marcel, T. C., Hartmann, F. E., Ma, X., Plissonneau, C., Zala, M., Ducasse, A., Confais, J., Compain, J., Lapalu, N., Amselem, J., McDonald, B. A., Croll, D., and Palma-Guerrero, J. 2017. A small secreted protein in Zymoseptoria tritici is responsible for avirulence on wheat cultivars carrying the Stb6 resistance gene. New Phytologist 31:166. 

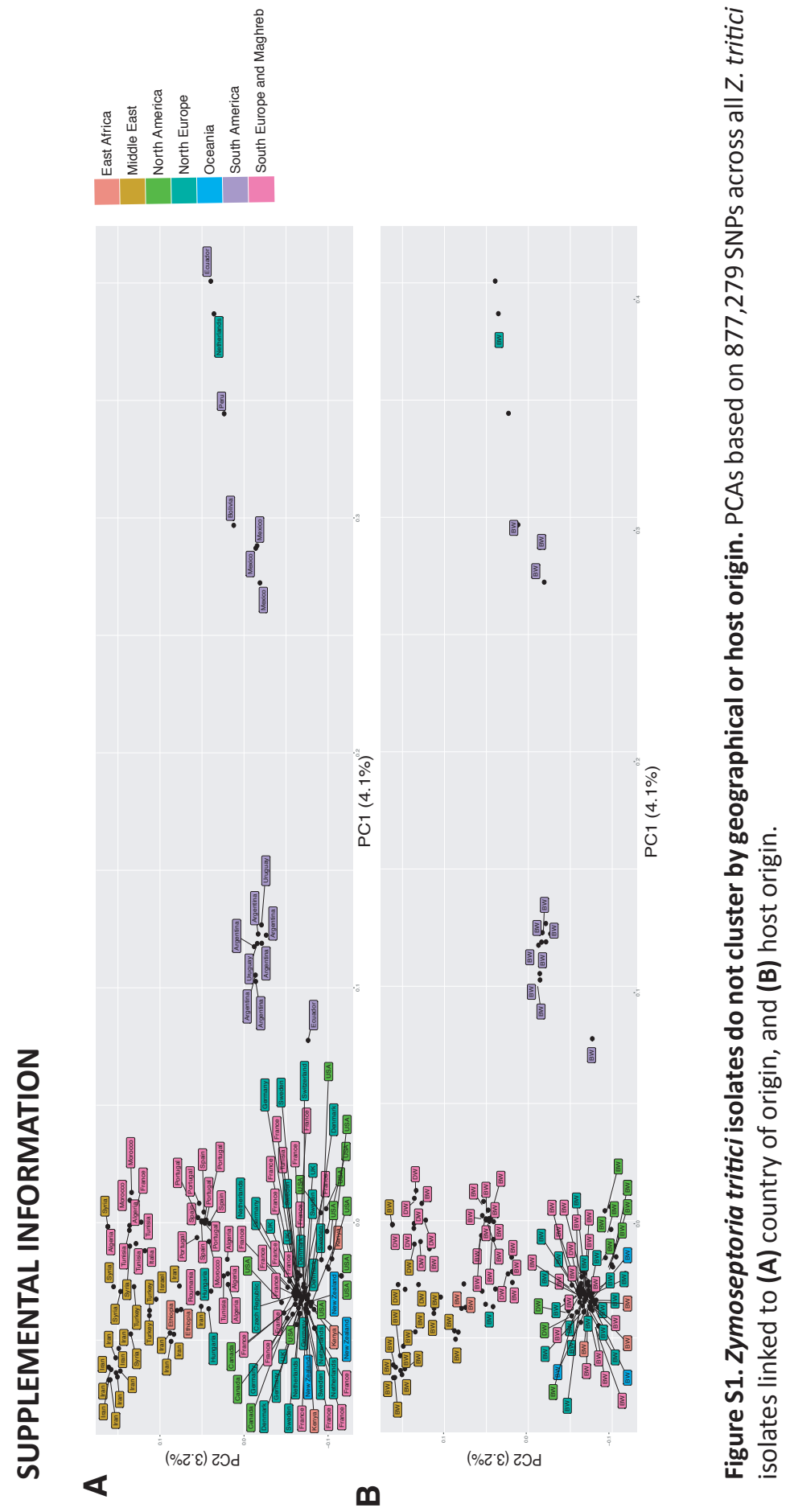


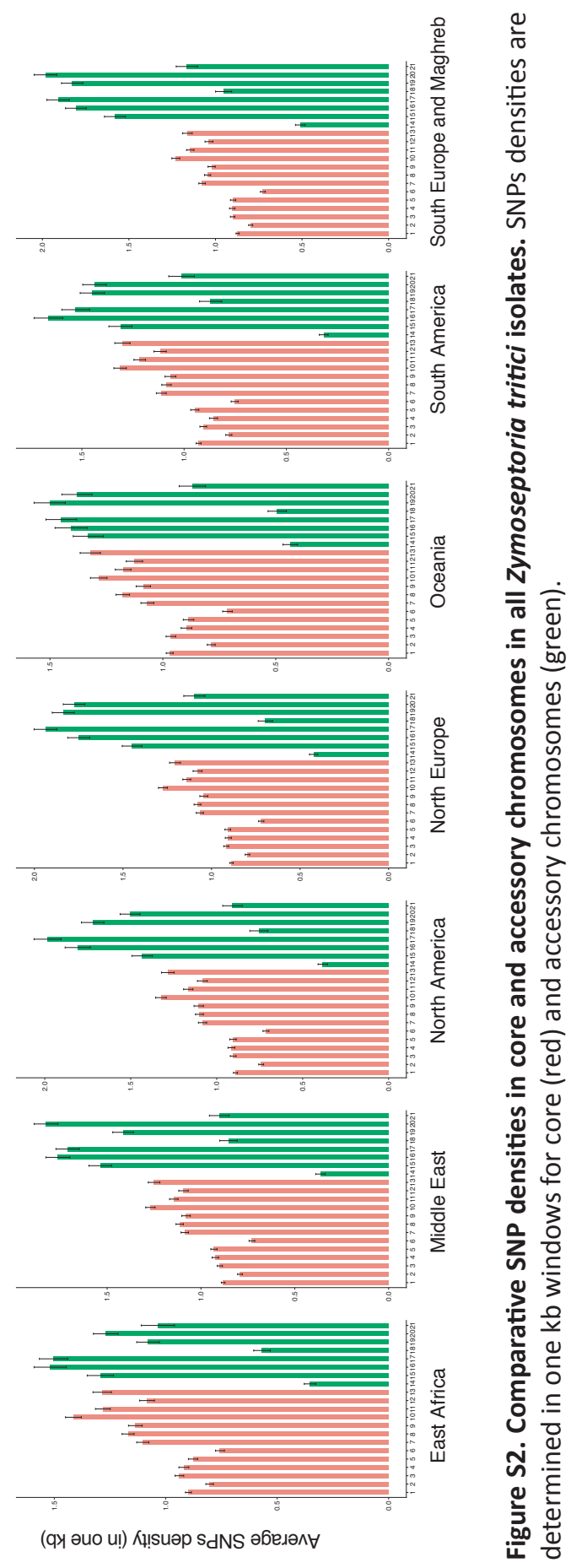




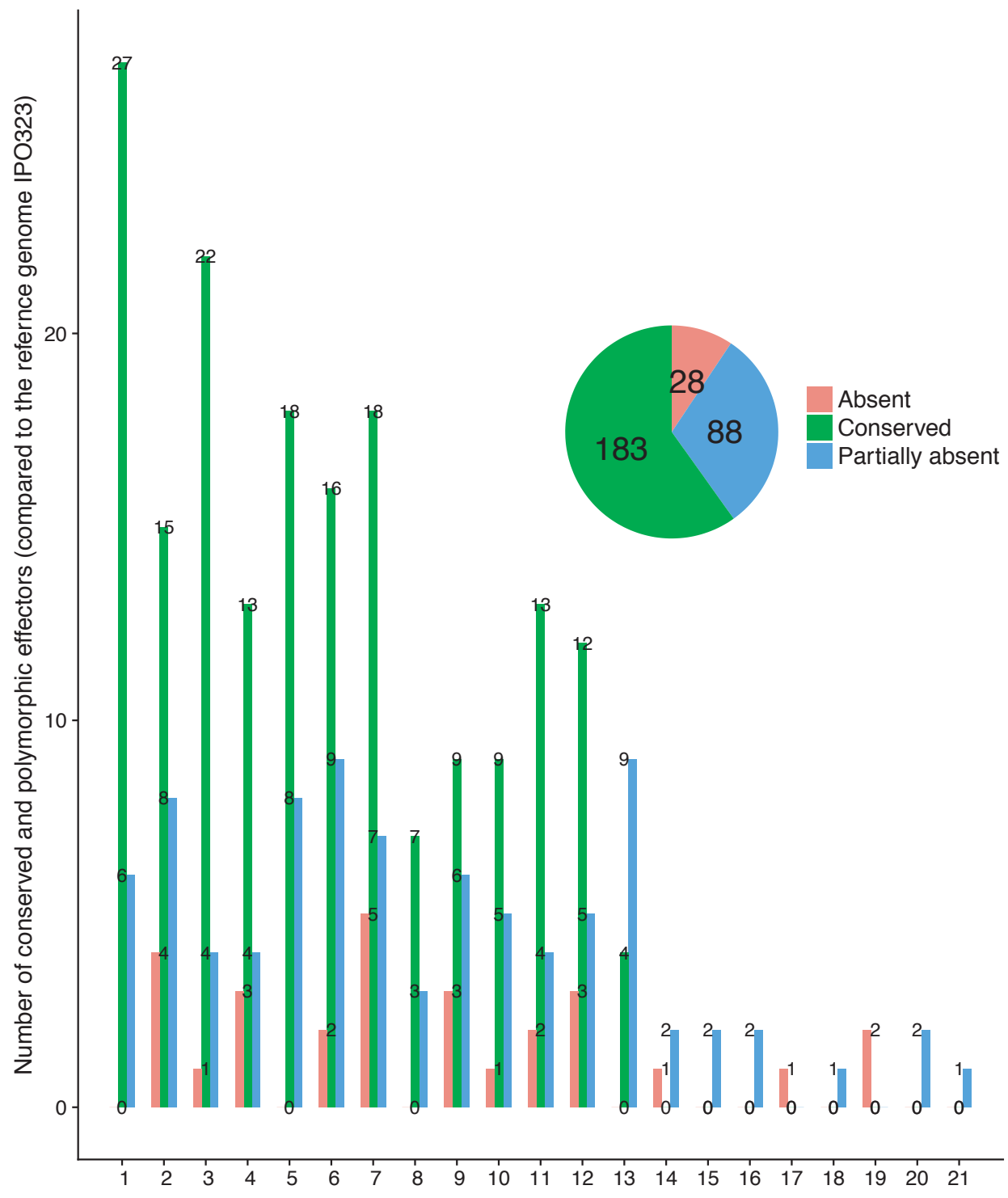

Figure S3. Abundance of conserved effectors in the global Zymoseptoria tritici panel. The pie chart shows the total number of conserved, partially absent, and absent effectors. Bar plots indicate the number of effectors per class on each chromosome. 


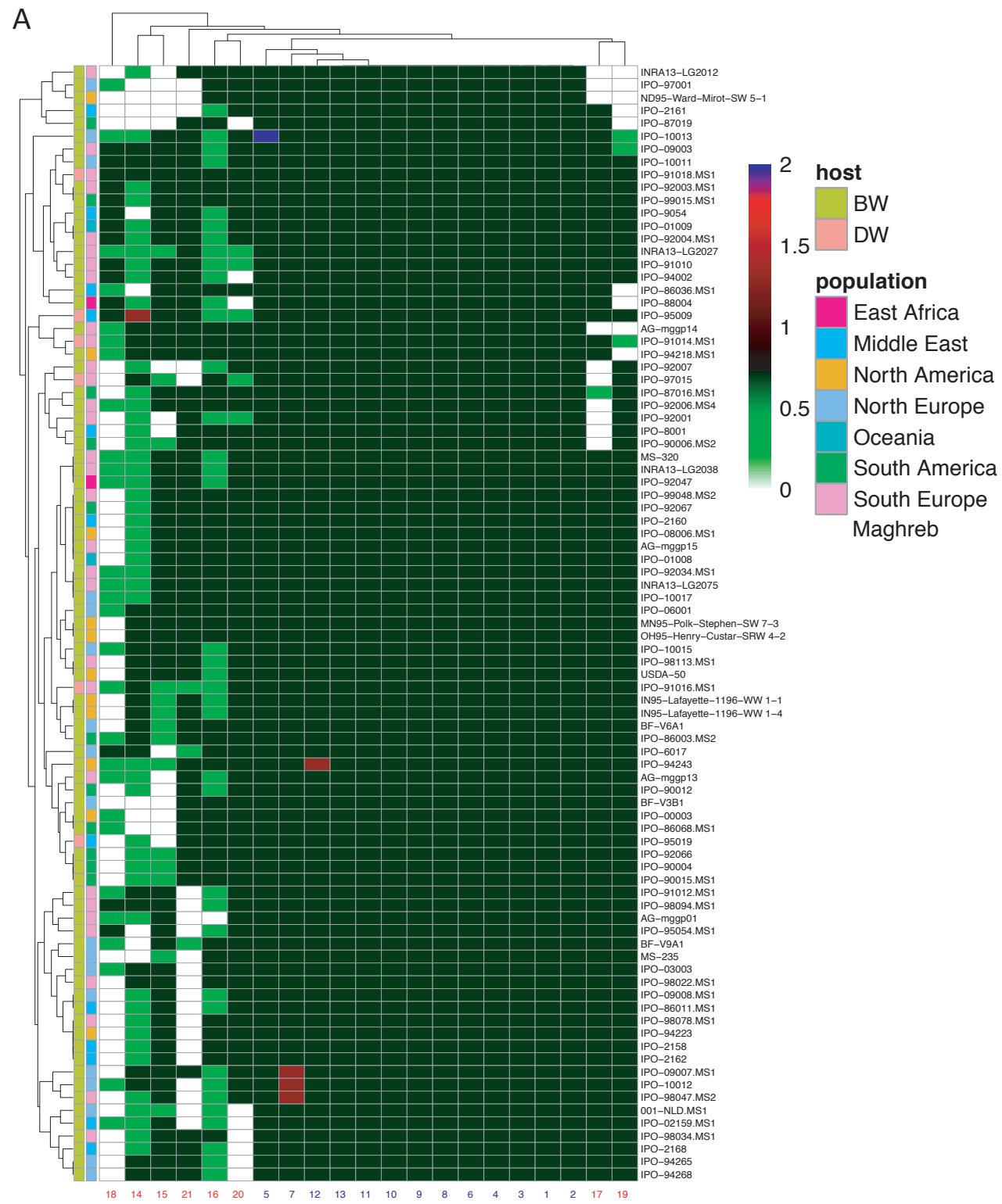

\section{Chromosomes}

Figure S4. Presence/absence chromosomal polymorphisms in Zymoseptoria tritici isolates adapted to bread or durum wheat. (A) Heatmap indicating presence/absence polymorphisms in each chromosome in bread and durum wheat isolates. Colors indicate presence (black), partially duplicated (red), duplicated (red), partially deleted (green) or absent (white). 
B
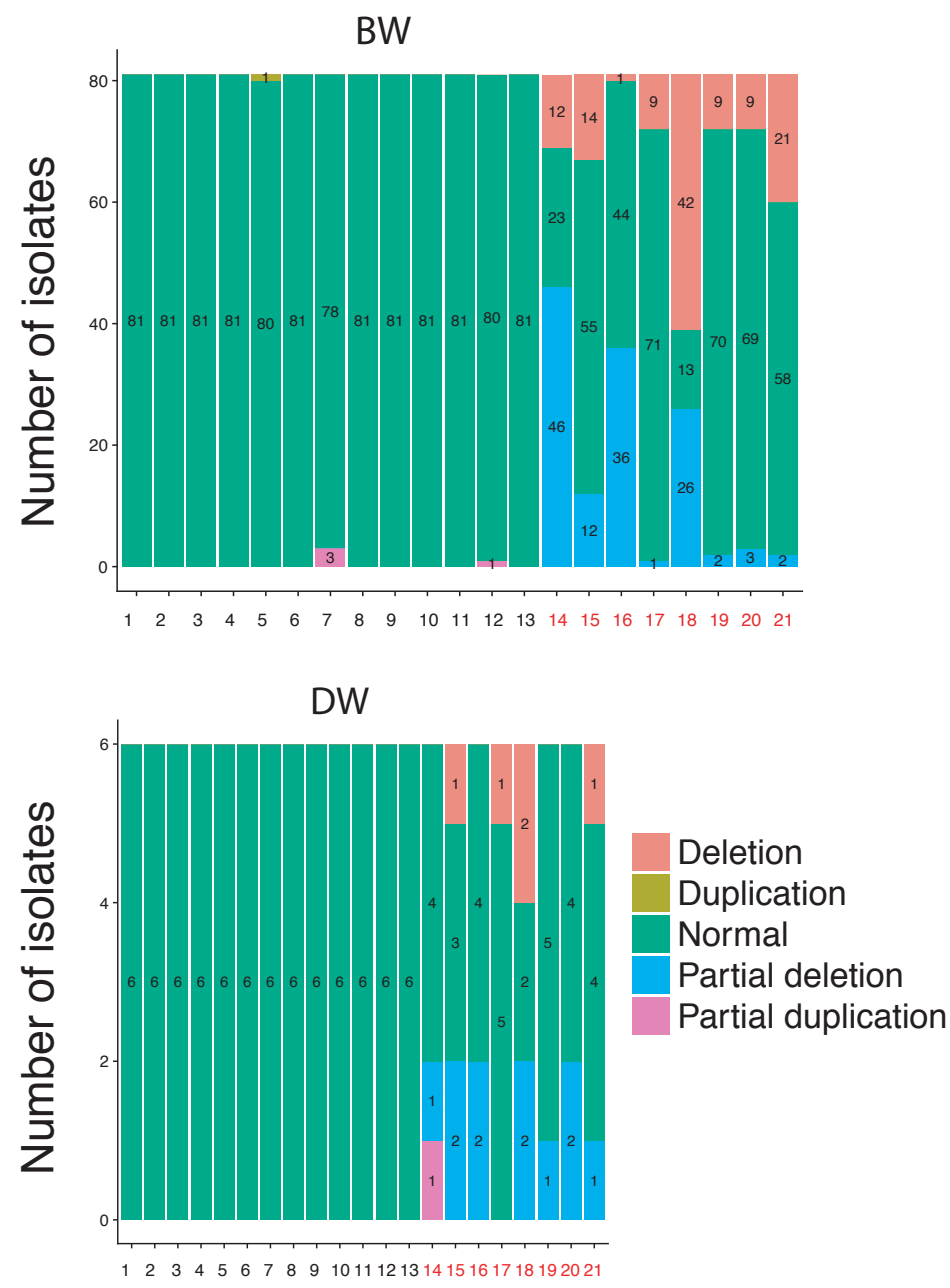

Figure S4 continued. (B) The frequencies of presence, partial duplication, duplication, partial deletion, and deletion are shown for each of the 21 chromosomes depicted as vertical bars. (C) Heatmap indicating the presence (black) and absence (white) of effectors in bread and durum wheat $Z$. tritici isolates based on IPO323 gene annotation. The identified Z. tritici effector AvrStb6 is indicated (green color). 
C

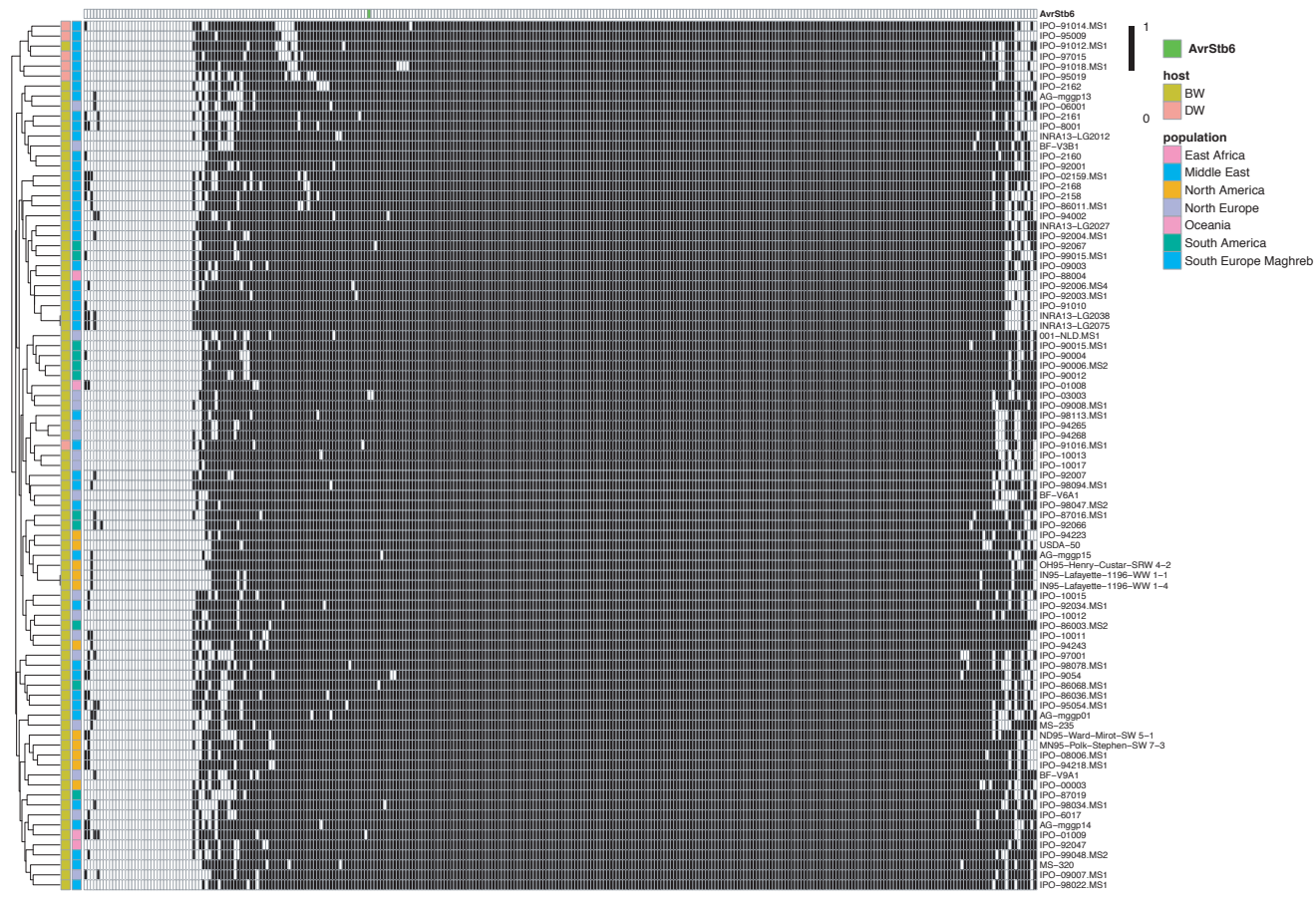


Table S1. Z. tritici isolates used in this study.

\begin{tabular}{|c|c|c|c|}
\hline Isolate & Country & Population & Host origin \\
\hline 001-NLD.MS1 & Netherlands & North Europe & BW \\
\hline AG-mggp01 & France & South Europe and Maghreb & BW \\
\hline AG-mggp12 & France & South Europe and Maghreb & BW \\
\hline AG-mggp13 & France & South Europe and Maghreb & BW \\
\hline AG-mggp14 & France & South Europe and Maghreb & BW \\
\hline AG-mggp15 & France & South Europe and Maghreb & BW \\
\hline BF-V1.1 & UK & North Europe & BW \\
\hline BF-V3B1 & UK & North Europe & BW \\
\hline BF-V6A1 & UK & North Europe & BW \\
\hline BF-V9A1 & UK & North Europe & BW \\
\hline Hung2.MS1 & Hungaria & North Europe & NA \\
\hline IN95-Lafayette-1196-WW 1-1 & USA & North America & NA \\
\hline IN95-Lafayette-1196-WW 1-4 & USA & North America & NA \\
\hline INRA08-FS0001.MS1 & France & South Europe and Maghreb & BW \\
\hline INRA08-FS0002.MS2 & France & South Europe and Maghreb & BW \\
\hline INRA08-FS0003.MS1 & France & South Europe and Maghreb & BW \\
\hline INRA13-LG2012 & Spain & South Europe and Maghreb & BW \\
\hline INRA13-LG2027 & Spain & South Europe and Maghreb & BW \\
\hline INRA13-LG2038 & Spain & South Europe and Maghreb & BW \\
\hline INRA13-LG2075 & Spain & South Europe and Maghreb & BW \\
\hline IPO-00003 & USA & North America & BW \\
\hline IPO-00005 & USA & North America & BW \\
\hline IPO-01008 & New Zealand & Oceania & BW \\
\hline IPO-01009 & New Zealand & Oceania & BW \\
\hline IPO-02159.MS1 & Iran & Middle East & BW \\
\hline IPO-02166.MS1 & Iran & Middle East & BW \\
\hline IPO-03001 & Germany & North Europe & BW \\
\hline IPO-03003 & Germany & North Europe & BW \\
\hline IPO-052462 & NA & NA & NA \\
\hline IPO-06001 & Denmark & North Europe & BW \\
\hline IPO-06008 & Denmark & North Europe & BW \\
\hline IPO-08002 & Iran & Middle East & BW \\
\hline IPO-08004 & Canada & North America & DW \\
\hline
\end{tabular}


Table S1. continued

\begin{tabular}{|c|c|c|c|}
\hline Isolate & Country & Population & Host origin \\
\hline IPO-08006.MS1 & Canada & North America & DW \\
\hline IPO-09003 & France & South Europe and Maghreb & BW \\
\hline IPO-09005 & France & South Europe and Maghreb & BW \\
\hline IPO-09007.MS1 & Germany & North Europe & BW \\
\hline IPO-09008.MS1 & Germany & North Europe & BW \\
\hline IPO-09009.MS1 & Germany & North Europe & BW \\
\hline IPO-10011 & Sweden & North Europe & NA \\
\hline IPO-10012 & Sweden & North Europe & NA \\
\hline IPO-10013 & Sweden & North Europe & NA \\
\hline IPO-10014 & Sweden & North Europe & NA \\
\hline IPO-10015 & Sweden & North Europe & NA \\
\hline IPO-10017 & Sweden & North Europe & NA \\
\hline IPO-1004 & New Zealand & Oceania & BW \\
\hline IPO-2158 & Iran & Middle East & BW \\
\hline IPO-2160 & Iran & Middle East & BW \\
\hline IPO-2161 & Iran & Middle East & BW \\
\hline IPO-2162 & Iran & Middle East & BW \\
\hline IPO-2164 & Iran & Middle East & BW \\
\hline IPO-2165 & Iran & Middle East & BW \\
\hline IPO-2168 & Iran & Middle East & BW \\
\hline IPO-6017 & Denmark & North Europe & BW \\
\hline IPO-6026 & Denmark & North Europe & BW \\
\hline IPO-8001 & Iran & Middle East & BW \\
\hline IPO-86002.MS1 & Bolivia & South America & BW \\
\hline IPO-86003.MS2 & Ecuador & South America & BW \\
\hline IPO-86011.MS1 & Turkey & Middle East & BW \\
\hline IPO-86013.MS1 & Turkey & Middle East & BW \\
\hline IPO-86015.MS1 & Morocco & South Europe and Maghreb & BW \\
\hline IPO-86022.MS1 & Turkey & Middle East & DW \\
\hline IPO-86036.MS1 & Israel & Middle East & NA \\
\hline IPO-86068.MS1 & Argentina & South America & BW \\
\hline IPO-87016.MS1 & Uruguay & South America & BW \\
\hline IPO-87019 & Uruguay & South America & BW \\
\hline IPO-88004 & Ethiopia & East Africa & BW \\
\hline
\end{tabular}


Table S1. continued

\begin{tabular}{|c|c|c|c|}
\hline Isolate & Country & Population & Host origin \\
\hline IPO-88018.MS1 & Ethiopia & East Africa & BW \\
\hline IPO-89011.MS1 & Netherlands & North Europe & BW \\
\hline IPO-90004 & Mexico & South America & BW \\
\hline IPO-90006.MS2 & Mexico & South America & NA \\
\hline IPO-90012 & Mexico & South America & BW \\
\hline IPO-90015.MS1 & Peru & South America & NA \\
\hline IPO-9010 & Iran & Middle East & NA \\
\hline IPO-9040 & Iran & Middle East & NA \\
\hline IPO-9054 & Iran & Middle East & NA \\
\hline IPO-91003.MS1 & Syria & Middle East & NA \\
\hline IPO-91004.MS1 & Syria & Middle East & DW \\
\hline IPO-91009.MS1 & Tunisia & South Europe and Maghreb & DW \\
\hline IPO-91010 & Tunisia & South Europe and Maghreb & DW \\
\hline IPO-91012.MS1 & Tunisia & South Europe and Maghreb & DW \\
\hline IPO-91014.MS1 & Tunisia & South Europe and Maghreb & DW \\
\hline IPO-91016.MS1 & Tunisia & South Europe and Maghreb & DW \\
\hline IPO-91018.MS1 & Morocco & South Europe and Maghreb & DW \\
\hline IPO-91020.MS1 & Morocco & South Europe and Maghreb & DW \\
\hline IPO-92001 & Portugal & South Europe and Maghreb & BW \\
\hline IPO-92002 & Portugal & South Europe and Maghreb & BW \\
\hline IPO-92003.MS1 & Portugal & South Europe and Maghreb & BW \\
\hline IPO-92004.MS1 & Portugal & South Europe and Maghreb & BW \\
\hline IPO-92006.MS4 & Portugal & South Europe and Maghreb & BW \\
\hline IPO-92007 & Portugal & South Europe and Maghreb & BW \\
\hline IPO-92034.MS1 & Algeria & South Europe and Maghreb & BW \\
\hline IPO-92044 & Kenya & East Africa & BW \\
\hline IPO-92047 & Kenya & East Africa & BW \\
\hline IPO-92062 & Kenya & East Africa & BW \\
\hline IPO-92064 & Argentina & South America & BW \\
\hline IPO-92066 & Argentina & South America & BW \\
\hline IPO-92067 & Argentina & South America & NA \\
\hline IPO-93014 & Argentina & South America & BW \\
\hline IPO-94002 & Algeria & South Europe and Maghreb & BW \\
\hline IPO-94203 & USA & North America & BW \\
\hline
\end{tabular}


Table S1. continued

\begin{tabular}{|c|c|c|c|}
\hline Isolate & Country & Population & Host origin \\
\hline IPO-94218.MS1 & Canada & North America & BW \\
\hline IPO-94223 & USA & North America & BW \\
\hline IPO-94234 & USA & North America & BW \\
\hline IPO-94243 & USA & North America & BW \\
\hline IPO-94265 & Netherlands & North Europe & BW \\
\hline IPO-94268 & Netherlands & North Europe & BW \\
\hline IPO-9455 & France & South Europe and Maghreb & BW \\
\hline IPO-95001 & Switzerland & North Europe & NA \\
\hline IPO-95009 & Syria & Middle East & BW \\
\hline IPO-95019 & Syria & Middle East & DW \\
\hline IPO-95036.MS1 & Syria & Middle East & BW \\
\hline IPO-95050 & Algeria & South Europe and Maghreb & DW \\
\hline IPO-95054.MS1 & Algeria & South Europe and Maghreb & BW \\
\hline IPO-95062 & Algeria & South Europe and Maghreb & DW \\
\hline IPO-97001 & Czech Republic & North Europe & BW \\
\hline IPO-97015 & Italia & South Europe and Maghreb & BW \\
\hline IPO-98022.MS1 & France & South Europe and Maghreb & BW \\
\hline IPO-98034.MS1 & France & South Europe and Maghreb & BW \\
\hline IPO-98046.MS2 & France & South Europe and Maghreb & BW \\
\hline IPO-98047.MS2 & France & South Europe and Maghreb & BW \\
\hline IPO-98078.MS1 & France & South Europe and Maghreb & BW \\
\hline IPO-98094.MS1 & France & South Europe and Maghreb & BW \\
\hline IPO-98113.MS1 & France & South Europe and Maghreb & BW \\
\hline IPO-98114 & Hungaria & North Europe & BW \\
\hline IPO-99015.MS1 & Argentina & South America & BW \\
\hline IPO-99032 & France & South Europe and Maghreb & NA \\
\hline IPO-99048.MS2 & France & South Europe and Maghreb & NA \\
\hline MN95-Polk-Stephen-SW 7-3 & USA & North America & NA \\
\hline MS-235 & Netherlands & North Europe & BW \\
\hline MS-270 & Ecuador & South America & NA \\
\hline MS-320 & Roumania & South Europe and Maghreb & NA \\
\hline ND95-Ward-Mirot-SW 5-1 & USA & North America & NA \\
\hline OH95-Henry-Custar-SRW 4-2 & USA & North America & NA \\
\hline Paskeville & USA & North America & NA \\
\hline USDA-50 & USA & North America & BW \\
\hline
\end{tabular}




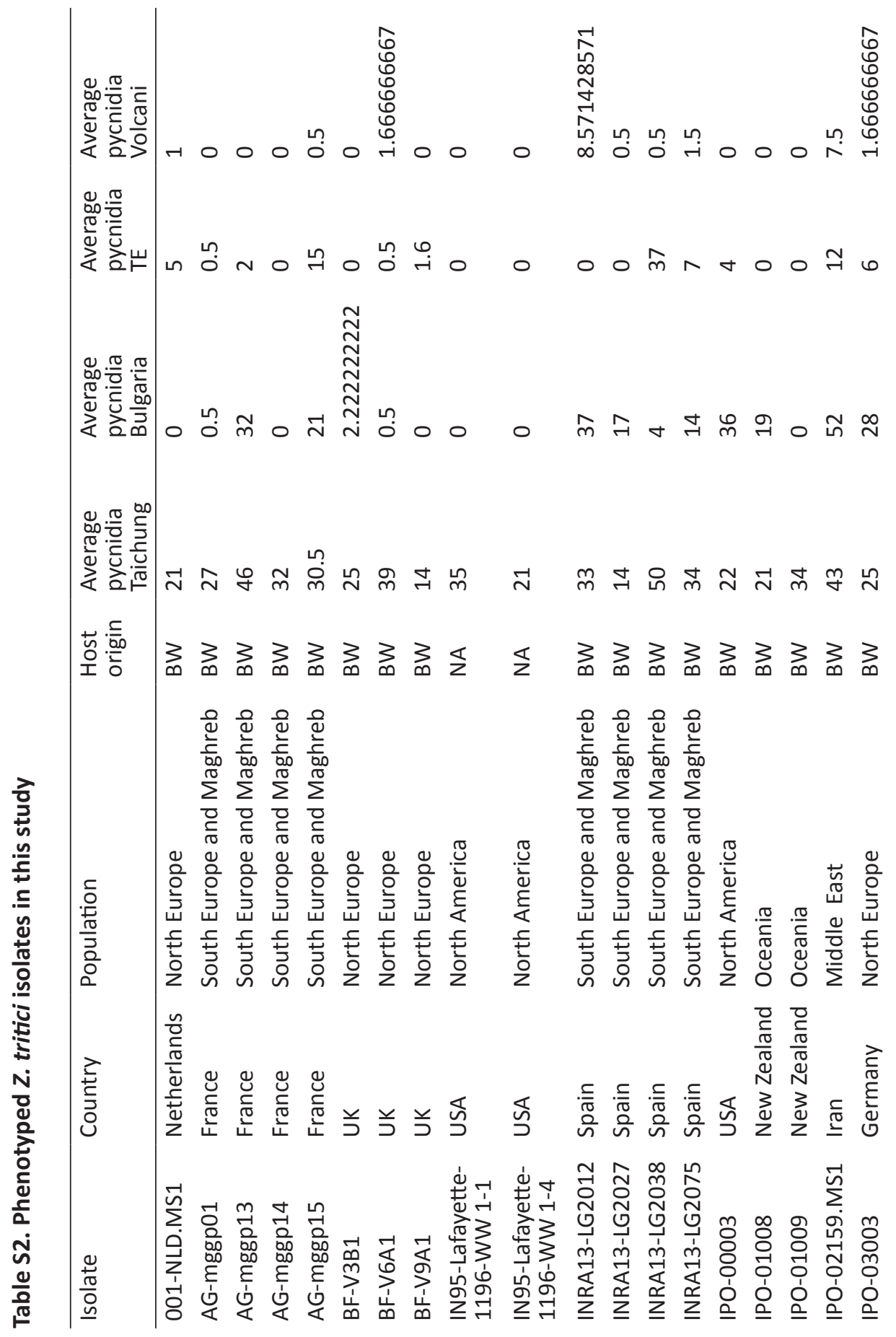




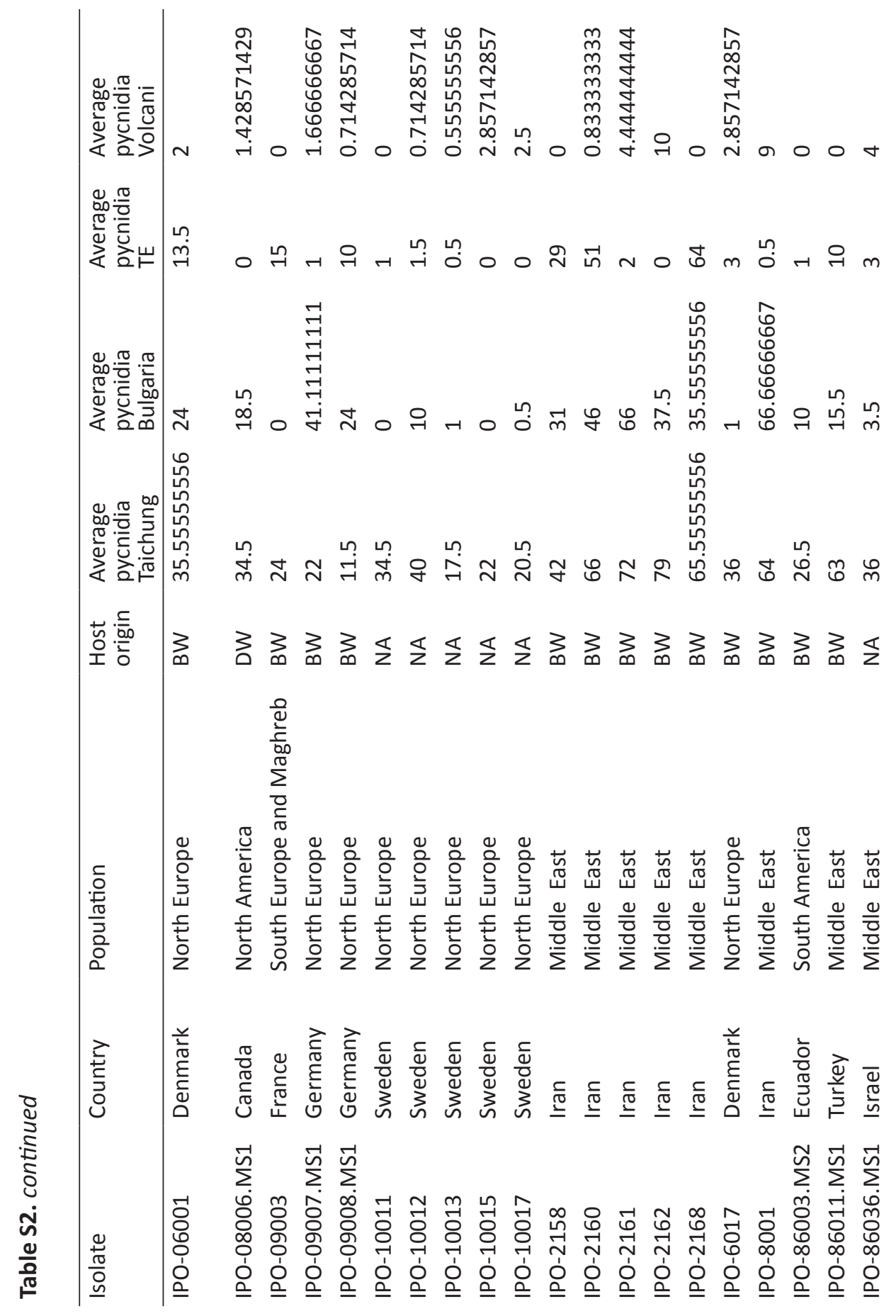




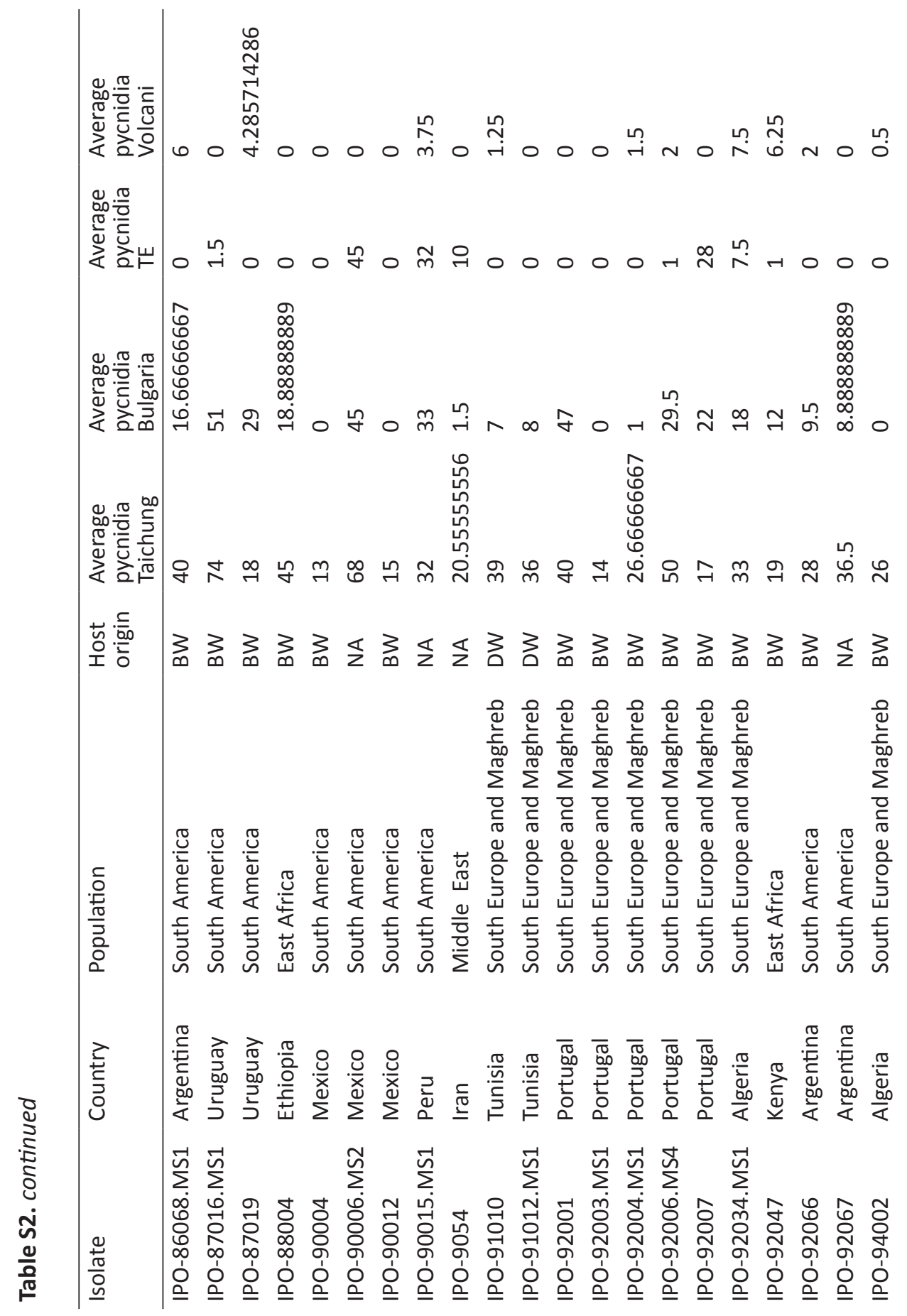




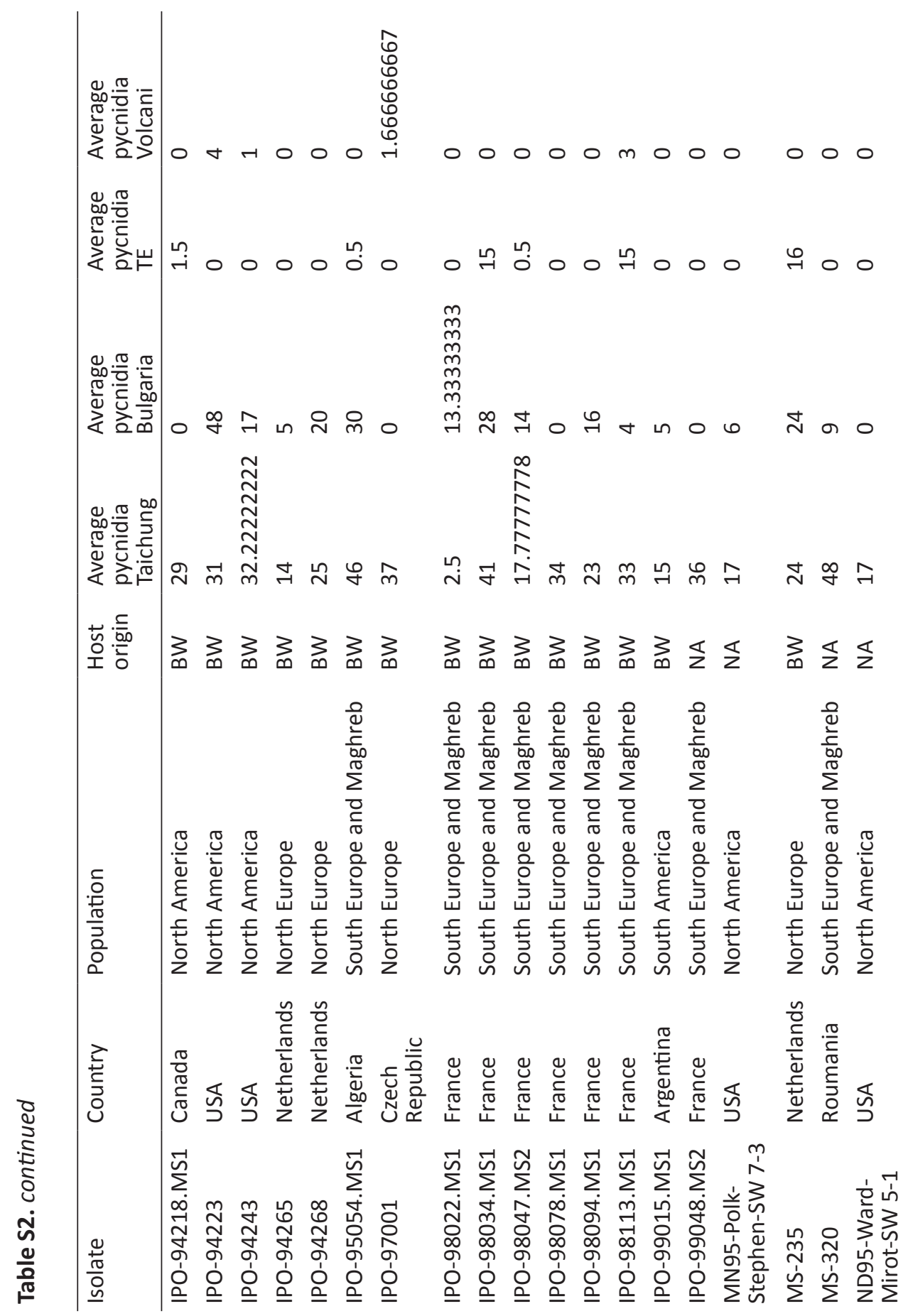




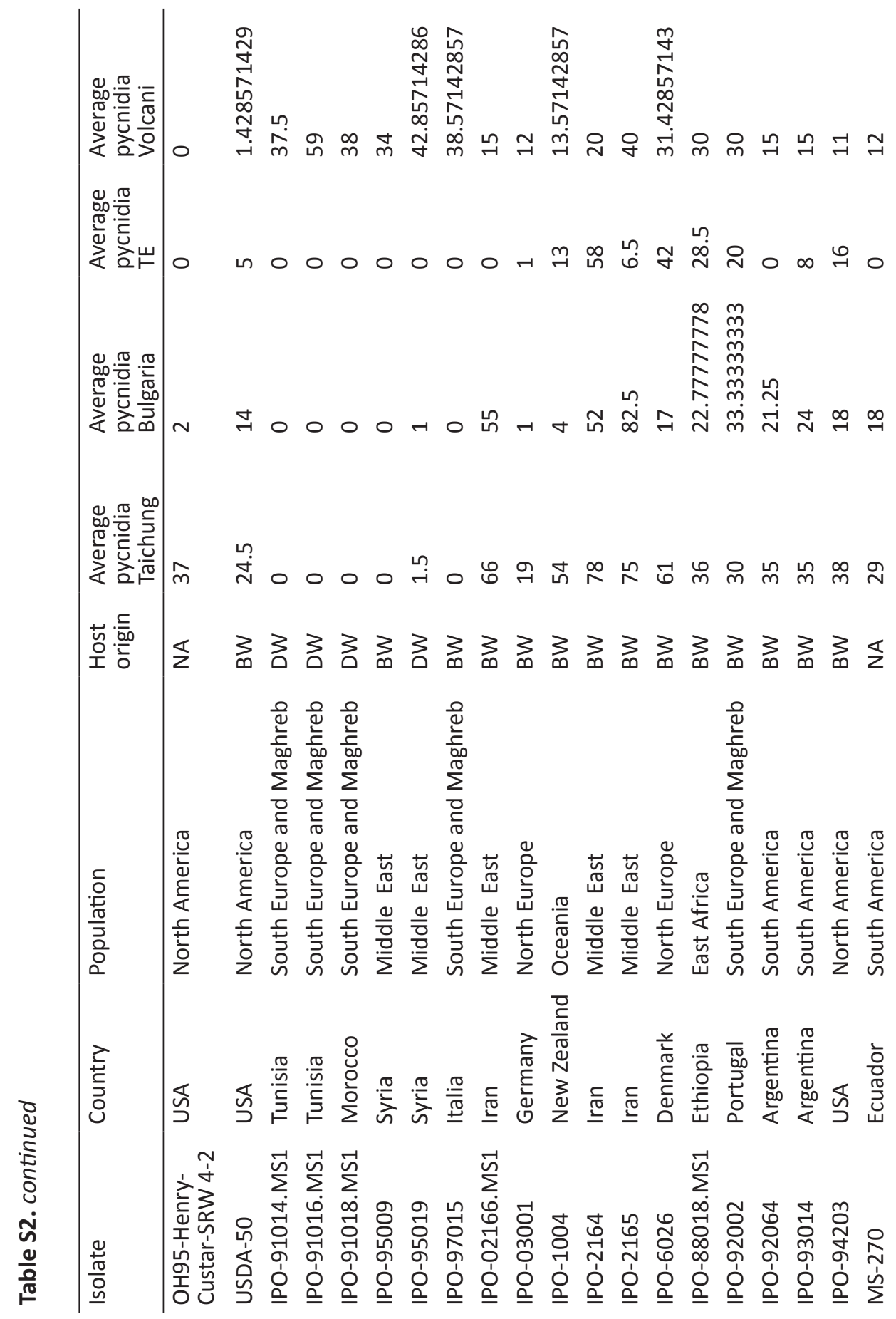




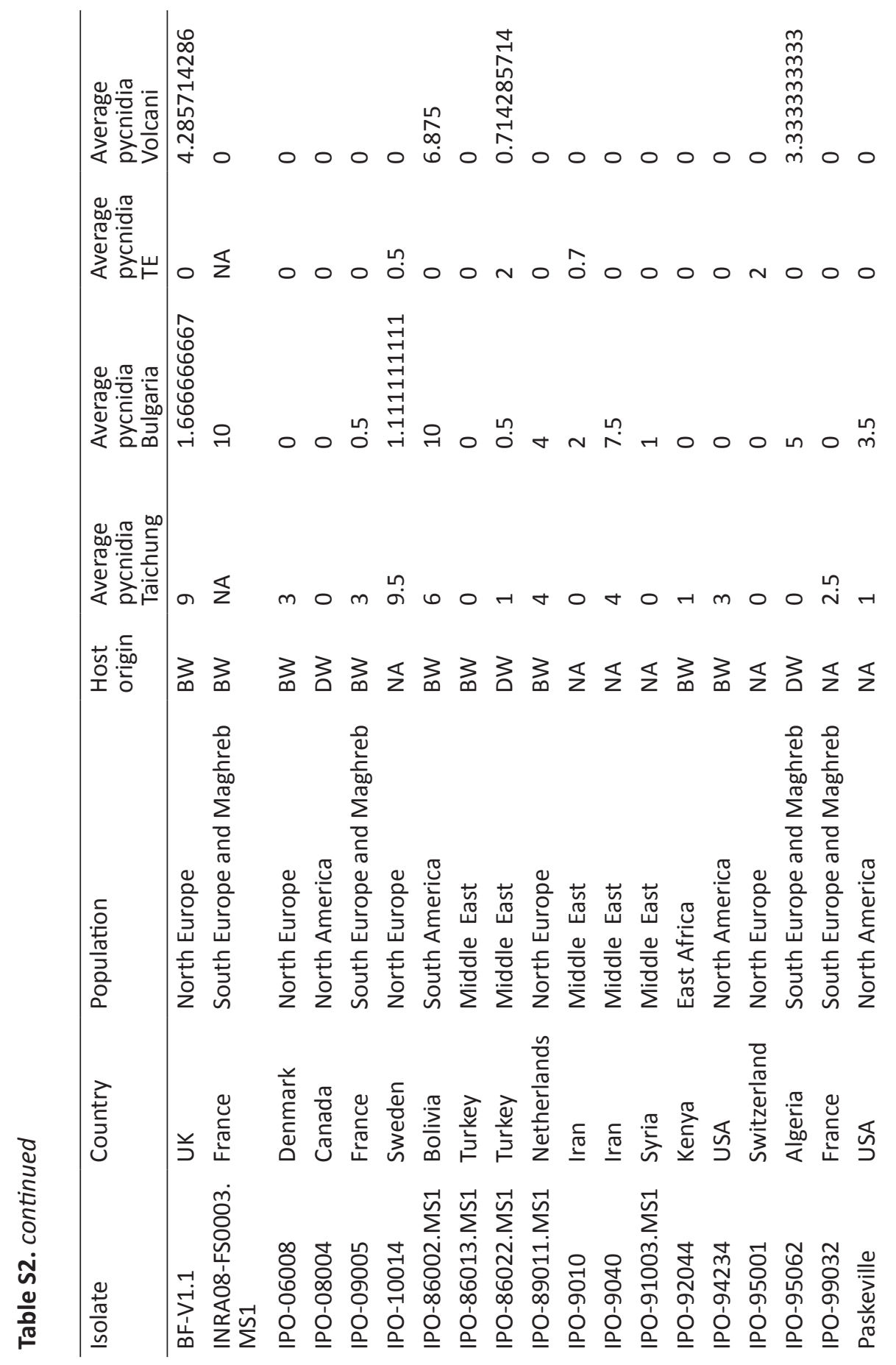




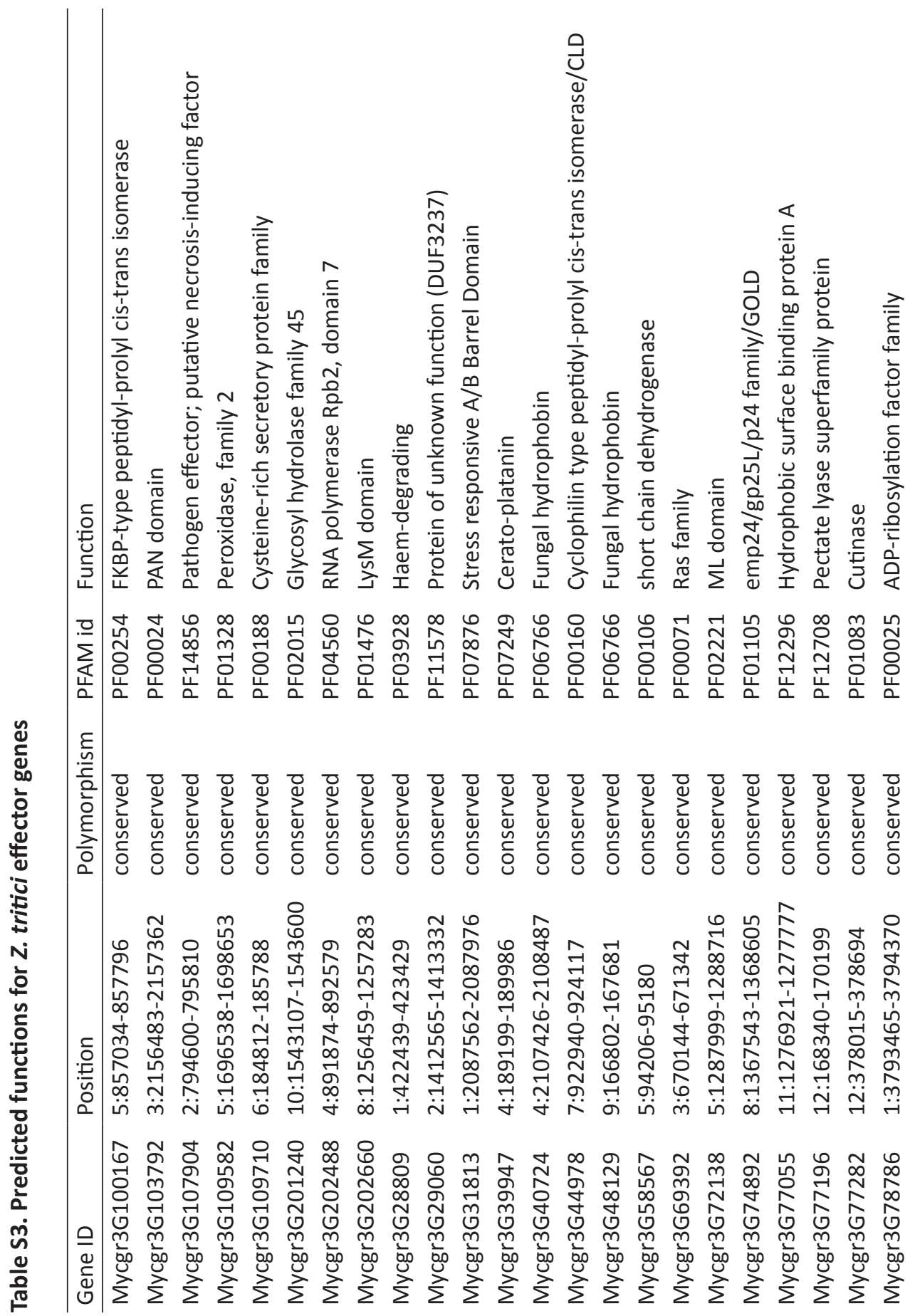




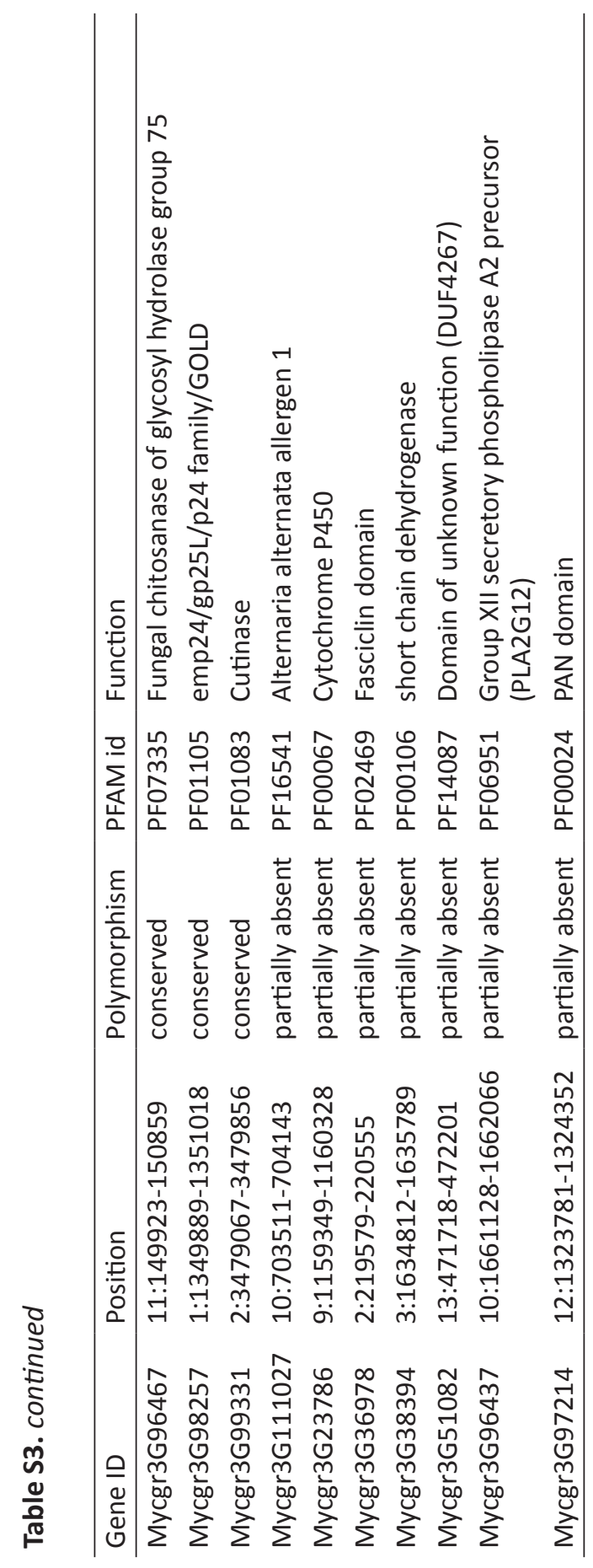




\section{Chapter 6}

$$
\text { do }
$$

$$
26
$$

ᄂ

$d \sigma$ 


\section{Stress and sexual reproduction affect the dynamics of the wheat pathogen effector AvrStb6 and strobilurin resistance}

Gerrit H.J. Kema ${ }^{1,2 \S}$, Amir Mirzadi Gohari ${ }^{1 \S}$, Lamia Aouini ${ }^{1 \S}$, Hesham Gibriel $^{28}$, Sarah Ware ${ }^{1^{*}}$, Frank Van den Bosch ${ }^{3}$, Robbie ManningSmith $^{3}$, Vasthi Alonso-Chavez ${ }^{3}$, Joe Helps ${ }^{3}$, Sarrah Ben M'Barek', Rahim Mehrabi ${ }^{5}$, Caucasella Diaz-Trujillo, ${ }^{1,2}$, Elham Zamani', Henk Schouten ${ }^{1}$, Theo van der Lee ${ }^{1}$, Cees Waalwijk¹, Maarten de Waard², Pierre de Wit ${ }^{2}$, Els Verstappen ${ }^{1}$, Bart P.H.J. Thomma², Harold J.G. Meijer ${ }^{1}$, and Michael F. Seidl ${ }^{2}$

1 Wageningen University and Research, Wageningen Plant Research, PO Box 16, 6700AA Wageningen, the Netherlands.

2 Wageningen University and Research, Laboratory of Phytopathology, PO box 16, 6700AA Wageningen, the Netherlands.

3 Rothamsted Research, West Common, Harpenden, Hertfordshire, AL5 2JQ, United Kingdom.

4 Biotechnology Center of Borj Cedria (CBBC), Laboratory of Molecular Plant Physiology, P.O. Box 901, 2050 Hammam-Lif, Tunisia.

5 Department of Agricultural Biotechnology, College of Agriculture, Isfahan University of Technology, Isfahan, Iran.

6 Department of Plant Pathology, Tarbiat Modares University, Tehran, Iran.

\$ These authors contributed equally

Published in Nature Genetics 50:375-380 (2018) 


\begin{abstract}
Host resistance and fungicide treatments are cornerstones of plant-disease control. Here, we show that these treatments allow sex and modulate parenthood in the fungal wheat pathogen Zymoseptoria tritici. We demonstrate that the $Z$. tritici-wheat interaction complies with the gene-for-gene model by identifying the effector AvrStb6, which is recognized by the wheat resistance protein Stb6. Recognition triggers host resistance, thus implying removal of avirulent strains from pathogen populations. However, Z. tritici crosses on wheat show that sex occurs even with an avirulent parent, and avirulence alleles are thereby retained in subsequent populations. Crossing fungicide-sensitive and fungicide-resistant isolates under fungicide pressure results in a rapid increase in resistance-allele frequency. Isolates under selection always act as male donors, and thus disease control modulates parenthood. Modeling these observations for agricultural and natural environments reveals extended durability of host resistance and rapid emergence of fungicide resistance. Therefore, fungal sex has major implications for disease control.
\end{abstract}




\section{INTRODUCTION}

Sexual reproduction is common in nearly all branches of the eukaryotic tree of life, including microbial organisms like fungi (Baret et al. 2008; Peterson et al. 2014), and has been considered an important driver for rapid adaption to novel or changing environments (Seidl et al. 2014). Dothideomycete fungi represent the largest and most ecologically diverse group of ascomycetes with approximately 20,000 species (Goodwin et al. 2011), and most of them reproduce sexually and asexually. One of them is the plant pathogen Zymoseptoria tritici that causes septoria tritici blotch in wheat. At the onset of the wheat growing season, Z. tritic produces air-borne sexual ascospores, thereby releasing genetically diverse founding populations in commercial wheat fields (Hunter et al. 1999; Kema et al. 1996a; Shaw et al. 1989), and splash-dispersed asexual conidia that drive epidemics during the growing season (Shaw et al. 1993). Fungicides and host resistance are paramount for disease control. Until now, 21 resistance genes to septoria tritici blotch (Stb genes) have been identified (Table S1) and mapped, and Stb6, which is ubiquitous in European wheat cultivars (Chartrain et al. 2005), is the first resistance gene that was recently cloned (Saintenac et al. 2018). However, the molecular processes underlying the $Z$. tritici-wheat interaction are still relatively poorly understood (Linde et al. 2002; Mehrabi et al. 2009; Thrall et al. 2015).

\section{MATERIALS AND METHODS}

\section{Primer development and PCR conditions}

We developed a mismatch amplification mutation assay (MAMA) (Cha et al. 1992) on part of the cytochrome $b(c y t b)$ gene to determine azoxystrobin sensitivity or resistance among generated Zymoseptoria tritici ascospore progenies. Primers were designed with a mismatch on the penultimate nucleotide and the ultimate nucleotide was at position 143 of $c y t b$. The primer set to specifically amplify a DNA fragment in sensitive isolates used a sense primer StrobSNP2fwd [5'-3' (404-428)] with a mismatch of T instead of G at nucleotide 427 of $c y t b$ and an antisense primer StrobSNP1rvs [5'-3' (1024-1043)]. The primer set to specifically amplify a DNA fragment in resistant isolates used an antisense primer StrobSNPrcF7[5'-3'(428-453)] with a mismatch of T instead of $G$ at nucleotide 429 and a sense primer StrobSNPrcR1 [5'-3' (152-173)]. One and $0.5 \mu$ of DNA were used for the MAMA and mating-type PCR assays, respectively.

Mating type PCR primers and thermal cycling conditions were as previously described (Waalwijk et al. 2002). Amplicons were analysed on $1.2 \%$ agarose gels using $25 \mu \mathrm{l}$ aliquots of the PCR products. PCRs to amplify simple sequence repeats (SSRs) were in a $20 \mu$ l volume containing $20 \mathrm{ng}$ DNA, $2 \mu \mathrm{l}$ 10X PCR buffer with $\mathrm{MgCl}^{2+}, 2 \mu$ leach forward and reverse primers $(2 \mu \mathrm{M}), 0.8 \mu \mathrm{l}$ dNTPs $(5 \mathrm{mM}), 0.2 \mu \mathrm{l}$ Taq DNA polymerase $(5 \mathrm{U} / \mu \mathrm{l})$, and $x \mu$ l sdd water. Thermal cycling was as follows: cycle $1 ; 94^{\circ} \mathrm{C}$ for 2 mins., cycle 2 (repeated $12 \mathrm{x}$ ); $94^{\circ} \mathrm{C}$ for 30 secs. then $66^{\circ} \mathrm{C}$ for 30 secs. minus $1^{\circ} \mathrm{C}$ per cycle, then $72^{\circ} \mathrm{C}$ 
for 30 secs., cycle 3 (repeated $27 \mathrm{x}$ ); $94^{\circ} \mathrm{C}$ for 30 secs., then $53^{\circ} \mathrm{C}$ for 30 secs., then $72^{\circ} \mathrm{C}$ for 30 secs. and cycle $4 ; 72^{\circ} \mathrm{C}$ for 7 mins., followed by a cooling-off step to $10^{\circ} \mathrm{C}$. Fragments were separated on a Mega-Gel Dual High-Throughput Vertical Electrophoresis Unit (CBS Scientific, Del Mar, CA, USA) with 6\% non-denaturing acrylamide gels stained with ethidium bromide during the run.

To monitor biomass of isolates in crossing and infection assays, we designed specific TaqMan ${ }^{\circledR}$ probe/primer combinations for quantitative PCRs (qPCR) based on the mat11 and mat1-2 idiomorph sequences of the two reference Z. tritici isolates IPO323 and IP094269, respectively (Waalwijk et al. 2002). Primers that specifically amplify DNA fragments in mat1-1 isolates were Mmat1F3/Mmat1R3, with a FAM-fluorescent probe IP3, and primers to specifically amplify DNA fragments in mat1-2 isolates were Mmat2F7/Mmat2R7, with a YY-fluorescent probe 2P4. Both quantitative real-time amplifications were performed in a single PCR on an Applied Biosystems 7500 Realtime PCR System (Foster City, CA, USA). Total reaction volumes were $25 \mu \mathrm{l}$, including $3 \mu \mathrm{l}$ DNA, $12.5 \mu$ l Premix Ex Taq ${ }^{\text {TM }}$ (2X) (TaKaRa, Shiga, Japan), $1 \mu$ l each forward and reverse primers $(6 \mu \mathrm{M}), 0.67 \mu \mathrm{l}$ for each probe $(5 \mathrm{mM}), 0.5 \mu$ l ROX Reference Dye II (50x), and $8.33 \mu \mathrm{l}$ ultraPURE ${ }^{\mathrm{TM}}$ nuclease-free water (Gibco, Paisley, Scotland). Thermal cycling was as follows: cycle $1 ; 50^{\circ} \mathrm{C}$ for 2 mins., cycle $2 ; 95^{\circ} \mathrm{C}$ for 10 mins., cycle 3 (repeated 39x); $95^{\circ} \mathrm{C}$ for 15 secs., then $60^{\circ} \mathrm{C}$ for 20 secs. Results were analysed using Sequence Detection Software version 1.2.3 (Applied Biosystems, Foster City, CA, USA). Standard curves from serial dilutions of known concentrations of pure fungal DNA of the six parental isolates plus the DNA from the reference isolates (Table S5) gave highly similar results in CT values. Therefore, serial dilutions of DNA from isolates IPO323 and IPO94269 were included in each TaqMan ${ }^{\circ}$ PCR run to calculate the unknown concentrations of fungal DNA in inoculated wheat seedlings. The standard curves had very high R2 values (0.9900.996) for all data points from $3 \mathrm{pg}$ to $30 \mathrm{ng}$ and, therefore, CT values within this range were reliable (data not shown). See Table S6 for all used probes and primers.

\section{Generation and analyses of segregating Zymoseptoria tritici populations} Crossing assays

We used an in planta crossing protocol for all mating assays (Kema et al. 1996a). For mapping, we extended the existing $Z$. tritici mapping population IPO323/IPO94269 to 400 progeny isolates and the IPO323/IP095052 population to 165 progeny isolates by manually collecting individual ascospores. For the EPP-biotic stress validation, we independently performed six crosses between avirulent and virulent isolates (IPO323, IP094269, IP095052) on five wheat cultivars (Obelisk, Shafir, Taichung 29, Inbar or Volcani 447) in multiple (>=2) biological replications. In addition, we used eight isolates in 19 crosses on nine wheat varieties (seven bread wheat and two durum wheat) and one barley accession (Tables S2 and 3) to test the occurrence of sex despite one of the parents is avirulent. For the EPP-abiotic stress validation we conducted 42 crosses between three sets of fungicide resistant and sensitive isolates on cv. Taichung 29 (Figure 3 and Figures S8 and 10; Table S5). Single sequence repeat (SSR) genotyping was 
routinely used to either confirm that segregating populations resulted from the applied parental isolates (Table 1, Figures S7 and 11) or to determine the genotype of asexual fructifications that appeared in crossing assays (Figures S12 and 13). Populations were maintained at $-80^{\circ} \mathrm{C}$ (Kema et al. 1996b) for further detailed analyses, including DArTSeq as well as MAMA, diagnostic PCRs for mating type determinations (Waalwijk et al. 2002) and the maternal/paternal contributions to sexual development, sequencing/ phenotyping to determine (a)virulence in progeny and wild type strains (Tables 1 and 2; Tables S4 and 5; Figure S8) and qPCR (Figure 3, Figures S8 and 10).

\section{Phenotyping}

We prepared inoculum following published procedures (Mirzadi Gohari et al. 2015) and performed seedling assays at growth stage (GS) 11-12 (Zadoks et al. 1974) either by painting a spore suspension using a soft brush (mapping populations) or by atomizing a spore suspension onto the potted seedlings that were placed at the perimeter of a circular rotary table in an inoculation cabinet, adjusted at $15 \mathrm{rpm}$, which is equipped with interchangeable atomizers and a water cleaning device to avoid cross-contamination between isolates (all other assays). Infected plants were incubated in transparent plastic bags for $48 \mathrm{~h}$ at $100 \% \mathrm{RH}$ in the aforementioned greenhouse. Disease severity was assessed at 21 days post-inoculation using necrosis and pycnidial development estimated as percentage of the total primary leaf area of individual seedlings. Following these procedures, we screened 190 IPO323/IP094269 offspring isolates, partly in three independent replicates (81 isolates) or singular tests (Figure S14) with the parental strains as controls, on cv. Shafir, carrying Stb6, and the susceptible control cv. Taichung 29.

\section{Genetic mapping}

Fungal genomic DNA was isolated using a standard CTAB-chloroform protocol. The parents and off-spring ( $\mathrm{N}=282$ ) of the $Z$. tritici mapping population (IPO323/IPO94269) (Goodwin et al. 2011; Wittenberg et al. 2009), were assayed of which 171 isolates showed distinct avirulence/virulence phenotypes on cv. Shafir. We used DArTSeq ${ }^{\mathrm{TM}}$, a genotyping-by-sequencing (GBS) method that combines diversity-arrays-technology (DArT) and next generation sequencing platforms (Courtois et al. 2013). In total 5,392 polymorphic DArTSeq markers Z. tritici isolates were obtained. Marker sequences (max $69 \mathrm{nt}$ ) were placed on the Z. tritici reference genome (Figure 1), using NCBI BLASTn (megablast) (Morgulis et al. 2008) and visualized using the GViz package (Hahne et al. 2013) (Figure 1). Multi-mapping markers were only placed on the genome at the best position if there was a considerable difference in bit-scores (difference $\geq 5$ ).

For fine mapping, the 5,392 generated DArTSeq markers were sorted according to their discrimination power for avirulent/virulent isolates by calculating the squared differences of genotype frequencies, and 60 DArTSeq markers linked with avirulence were selected. These markers were sorted into a genetic linkage map, using JoinMap ${ }^{\circ} 4$ software with settings LOD (Log of Odds) $\geq 3$ for grouping, and the maximum likelihood 
mapping option for linkage group generation (Van Ooijen et al. 2006). Since the segregation of avirulence fitted the model of single gene inheritance (Figure S14) (Kema et al. 2000), phenotypic data were converted to an appropriate marker (AvrStb6) using scoring codes that are required for JoinMap, and this was integrated in the mapping procedure.

Offspring isolates with more than $10 \%$ missing genotypic values were removed from the analysis. Moreover, isolates without recombination near the (a)virulence locus, and eight showing discrepancies between the genotyping and phenotyping were not considered for analysis. To delimit the physical region harboring AvrStb6, we deployed a graphical mapping approach using the recombinant offspring isolates and clustered the markers that co-segregated with AvrStb6 into bins with the marker order as estimated by JoinMap as a reference (Figure S15). The generated genetic linkage map was compared to the IPO323 reference genome sequence by aligning the DArTSeq data to determine the physical position of AvrStb6.

\section{Gene annotation}

Gene annotation was performed on the $Z$. tritici reference genome isolate IPO323 (Goodwin et al. 2011) using the Maker2 pipeline (Holt et al. 2011), combining ab initio protein-coding gene evidence from SNAP (Johnson et al. 2008), Augustus (Stanke et al. 2004), and GeneMark-HMM (Lukashin et al. 1998). Additionally, Maker2 was provided with protein alignments to 35 predicted fungal proteomes, Z. tritici reference gene models annotated by the Joint Genome Institute (JGI) (Goodwin et al. 2011), and transcriptome data (assembled transcripts and splice-junctions) derived from two previously published RNA-seq datasets (Rudd et al. 2015; Kellner et al. 2014). For gene annotation, RNA-seq data (single-end) were mapped to the $Z$. tritici reference genome with TopHat (version 2.0.13) (--min-intron-length 20 --max-intron-length 2000 --maxmultihits 5) (Trapnell et al. 2009). Z. tritici transcripts were assembled using Cufflinks (Trapnell et al. 2010). Gene models predicted with Maker2 were manually evaluated and refined (Lee et al. 2013), for example by excluding protein-encoding genes $<60$ aa or lacking a starting methionine.

\section{Identification of effector candidates}

Gene expression, expressed as fragments per kilobase of exon per million fragments mapped (FPKM), during wheat colonization for newly predicted protein-coding genes was inferred using Cuffdiff (version 2.2.1) (Trapnell et al. 2010). Similar to previous observations (Rudd et al. 2015; Palma-Guerrero et al. 2016), the third replicate of the RNA-seq experiment of Rudd et al. (2015) behaved differently and was therefore excluded from all further analyses. Pair-wise log2-fold expression changes as well as multiple-testing corrected $p$-values $(P<0.05)$ were inferred for in planta RNAseq samples compared to CDB (Rudd et al. 2015). N-terminal secretion signals were predicted in all proteins using SignalP (version 4.1) (Emanuelsson et al. 2007). Protein domains were predicted with InterProScan (Zdobnov et al. 2001). 


\section{Functional analyses of AvrStb6}

Strains, media and growth conditions

Z. tritici strains IPO323 and IPO94269, which are avirulent and virulent on cv. Shafir, were used as wild type strains (WTs) and recipient strains for gene deletion and ectopic expression (Figure S16). The WTs and all deletion strains were kept at $-80^{\circ} \mathrm{C}$ and were re-cultured on potato dextrose agar (PDA) (Sigma-Aldrich Chemie, Steinheim, Germany) at $15^{\circ} \mathrm{C}$ once desired for experimentation. Yeast-like spores were produced in yeast glucose broth (YGB) medium (yeast extract $10 \mathrm{~g} . \mathrm{L}^{-1}$, glucose $30 \mathrm{~g} . \mathrm{L}^{-1}$ ) after placement in an orbital shaker (Innova 4430, New Brunswick Scientific, Nijmegen, The Netherlands) at $15^{\circ} \mathrm{C}$. For in vitro expression analyses in Z. tritici blastospores we used YGB and MM (Barratt et al. 1965) under similar conditions, whereas we adjusted the conditions in YGB to $25^{\circ} \mathrm{C}$ for expression in mycelium. Escherichia coli $\mathrm{DH} 5 \alpha$ was used for general plasmid transformation and Agrobacterium tumefaciens strain AGL-1 was used for all fungal transformations.

\section{Fungal transformation}

All transformations were performed using $A$. tumefaciens mediated transformation (ATMT) as described previously (Zwiers et al. 2001; Mehrabi et al. 2006). Genomic DNA of stable transformants was extracted according to standard protocols (Sambrook et al. 2006). For ectopic complementation, the same procedure was utilized with minor modifications, including the use of $250 \mu \mathrm{g} \mathrm{m} . \mathrm{L}^{-1}$ geneticin for the selection of mutants.

\section{$R N A$ isolation and $q R T-P C R$}

In vitro and in planta expression profiling of AvrStb6 was performed using quantitative real-time PCR (qRT-PCR). For in planta analyses, wheat cv. Shafir was inoculated, in triplicate, with the WT isolates as described (Mehrabi et al. 2006), and leaf samples were collected at seven hours post-inoculation, and subsequently at $1,2,4,8,12,16$ and $20 \mathrm{dpi}$, followed by flash freezing and grinding in liquid nitrogen using a mortar and pestle. Total RNA was extracted either from ground leaves or fungal biomass produced in YGB using the RNeasy plant mini kit (Qiagen, MA, USA). DNA contamination was removed with the DNAfree kit (Ambion, Cambridgeshire, U.K.). First-strand cDNA was synthesized from approximately $2 \mu \mathrm{g}$ of total RNA primed with oligo(dT) using the SuperScript III following manufacturers' instructions. One $\mu$ of the resulting cDNA was used in a $25 \mu \mathrm{l}$ PCR reaction using a QuantiTect SYBR Green PCR Kit and run and analysed using an ABI 7500 Real-Time PCR System. The relative expression of each gene was initially normalized with the constitutively expressed Z. tritici beta-tubulin gene (Keon et al. 2007) and then calculated based on the comparative $C(t)$ method described previously (Figure S2) (Schmittgen et al. 2008).

\section{Pathogenicity assays and quantitative fungal biomass analyses}

All assays were conducted as described above using wheat cvs. Shafir and Taichung 29 (Figures S5 and 17). Disease development was monitored and recorded every three days and leaves of cv. Taichung 29 were harvested at 2, 4, 8, 12, 16 and 20 dpi for 
qRT-PCR expression analyses and for qPCR fugal biomass determination of all WTs and transformed Z. tritici strains (Waalwijk et al. 2002; Ware 2006) (Figure S17). Genomic DNA was extracted from approximately $100 \mathrm{mg}$ of infected leaves using a standard phenol/chloroform DNA extraction (Sambrook et al. 2006).

\section{Generation of gene deletion and ectopic integration constructs}

To generate the AvrStb6 deletion construct, pKOZtAvrStb6, the multisite Gateway threefragment vector construction kit was used, enabling the cloning of three fragments into the destination vector, which was compatible with the ATMT procedure. A $2 \mathrm{~kb}$ upstream and downstream sequence of AvrStb6 was cloned in pDONR ${ }^{\text {TM }}$ 4-P1R and pDONR ${ }^{\mathrm{TM}} \mathrm{P} 2 \mathrm{R}-\mathrm{P} 3$. The generated constructs along with pRM250 (Mehrabi et al. 2009) containing the hygromycin phosphotransferase $(H p h)$ gene as a selection marker were cloned into the destination vector, pPm43GW, via the LR reaction. In order to make the AvrStb6 ectopic integration construct (pZtAvrStb6.com), the full ORF of AvrStb6, including a 1,020 bp upstream stretch as its promoter and $552 \mathrm{bp}$ stretch downstream

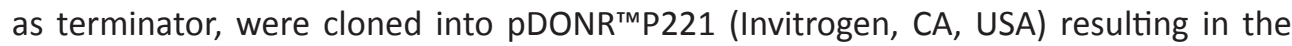
generation of p221-ZtAvrStb6.com. The p221-ZtAvrStb6.com as well as two entry vectors pRM245 and pRM234 (Mehrabi et al. 2009), were used to clone these three fragments into the destination vector, pPm43GW, through the LR reaction.

\section{Determining exclusive paternal parenthood}

EPP-biotic stress

To determine parenthood in the conducted crosses, we analysed four crosses (Table 1; Figures S6 and 7) using four markers (AvrStb6, mat, ag-0006 and mt-SSR) and monitored fungal biomass development by qPCR (Figure 3, Figure S8).

\section{EPP-abiotic stress}

Strobilurin sensitivity was assayed in six strains (Supplementary Table 6) on potato dextrose agar (PDA) plates that were amended with kresoxim-methyl (BASF, Ludwigshafen, Germany) and trifloxystrobin (Bayer CropScience, Monheim am Rhein, Germany) and determined minimal inhibitory concentrations (MICs) of two different technical samples of the fungicides by spotting isolates on strobilurin amended PDA plates. The concentrations for kresoxim-methyl were $0.0025,0.005,0.01,0.025,0.05$, $0.1,0.25,0.5$, and $1.0 \mathrm{ppm}$, and the concentrations for trifloxystrobin were 0.00025 , $0.0005,0.001,0.0025,0.005,0.01,0.025,0.05,0.1$ and $0.25 \mathrm{ppm}$. All isolates were spotted in triplicate in a volume of $5 \mu \mathrm{l}$ per spot at a concentration of $4 \times 10^{5}$ spores $\mathrm{ml}^{-1}$. As a positive control for growth isolates were also plated on PDA amended with the strobilurin solvent $\left(1 \%\right.$ methanol). Plates were placed at $18^{\circ} \mathrm{C}$ in the dark for 10 days, after which MIC values were assessed. A test progeny was generated by crossing Z. tritici isolates IPO03001 and IPO03003 and analysed it on amended PDA plates and with MAMA assays to conclude that both methods are congruous. 
MIC values for the six parental isolates (Table S5) for the commercially available fungicide Amistar ${ }^{\mathrm{TM}}$ (Syngenta, Roosendaal, Netherlands) were determined, containing the active ingredient azoxystrobin, at $0.1,1.0$, and $10 \mathrm{ppm}$ and then determined which concentrations of azoxystrobin to use for infection and crossing assays using an in planta dose response curves for the sensitive $Z$. tritici isolates using different preventive applications of azoxystrobin (250 g.L-1 a.i. of azoxystrobin; 50\% E.C.) on 10 day-old seedlings of cv. Taichung 29 that were preventatively treated (48h) using a track sprayer that was calibrated to deliver the recommended application of 1 L.ha $^{-1}$ sprayed at a rate of 250 L.ha $^{-1}$, with the following percentages of the full recommended dose: 0 , $3.125,6.25,12.5,25,50,100$ and $200 \%$ (which correspond with fungicide solutions of $0,0.03125,0.0625,0.125,0.25,0.5,1$, and $2 \mathrm{~g}$ azoxystrobin. $\mathrm{L}^{-1}$, respectively). We then inoculated with $Z$. tritici and percentages of leaf area covered by pycnidia were recorded at 20,23, 26, and 29 dpi for dose response curve experiments, at 20 dpi for infection assays, and at 0, 5, 10, 15, 20, 25 and 30 dpi for qPCR biomass monitoring over time (Figure 3, Figures S9 and 10). Finally, three sets of $Z$. tritici field isolates IPO03001/ IP003003, IPO03002/IP003005 and IP004001/IP004011, with equal pathogenicity, opposite mating types and contrasting sensitivity to azoxystrobin (Table 2, Figure 3 , Figure S9) were used for the generation of 42 in planta ascospore progenies and fungal biomass development of each isolate in each crossing assay (Figure 3, Figure S10) individually and in pairwise mixtures on untreated and preventatively treated (48h, 100\% azoxystrobin) seedlings of the wheat cv. Taichung 29 was monitored. Leaf samples were collected at 0,5, 10, 15, 20, 25 and $30 \mathrm{dpi}$ and were immediately frozen in liquid nitrogen before storage at $-80^{\circ} \mathrm{C}$ until lyophilization, subsequent DNA extraction, and qPCR analyses. Two extractions were made from each sample (technical repeats), and the mean results were expressed in ng of fungal DNA.mg ${ }^{-1}$ dry weight leaf material. A first set of 18 crosses was performed in seedlings of cv. Taichung 29 that were preventively treated (48h) with Amistar $^{\mathrm{TM}}$ at 0 (control), 3.125, 6.25, 12.5, 25 and $50 \%$ of the full rate. In a second set of 24 crosses we repeated these conditions, but added two concentrations; full rate (100\%), and the double rate (200\%) (Table 2, Table S5). From six through 12 weeks after inoculation, material was harvested for ascospore discharge and collection (Kema et al. 1996a). Ascospores were isolated as much as possible from diverse locations within a plate or within several plates from each cross to obtain random ascospore progenies. Baseline germination frequencies on unamended WA plates for all 42 progeny sets $(\mathrm{N}=15,975)$ and randomly selected ascospores were determined. Germination frequencies of the 24 ascospore progenies for the second series of crosses were also determined on WA amended with $1 \mathrm{ppm}$ active ingredient azoxystrobin $(\mathrm{N}=9,025)$, and these frequencies were expressed as percentages relative to the mean of the control germination frequencies on unamended water agar. We evaluated the percentage of resistant offspring by 2,100 independent MAMA PCRs (Table 2). 


\section{Developing the new population genetics model}

The model equations (Table S7) were numerically solved in $\mathrm{C}^{++}$. Output was plotted using the graphics package Sigmaplot. The calculation showing that, independent of the parameter values (see also Figure S18), the frequency of virulence increases slower when the avirulent strain takes part in the sexual reproduction was done by hand and checked using the package Maple. We modelled the population genetic consequences of this new observation using an allele frequency model as introduced by Leonard (Leonard et al. 1969).

\section{Data availability}

All data are available and deposited in NCBI Genbank under accession number ACPE00000000 (Goodwin et al. 2011), in Gene Expression Omnibus under the accession number GSE54874 (Kellner et al. 2014), and as a BioProject with the accession number PRJEB8798 (Rudd et al. 2015).

URL section DArTSeq http://genome.jgi.doe.gov/Mycgr3/Mycgr3.home.html

\section{RESULTS AND DISCUSSION}

Gene-for-gene (GFG) interaction models have been suggested for a plethora of plant-pathogen interactions (Thrall et al. 2015), but genetic proof was only provided for a limited number of pathosystems (Brading et al. 2002; Brown et al. 2011). After more than a decade of genetic studies (Goodwin et al. 2011; Kema et al. 1996a; Wittenberg et al. 2009; Kema et al. 2002; Kema et al. 2000; Mirzadi Gohari et al. 2015; Waalwijk et al. 2002) we report the map-based cloning of the first Z. tritici avirulence effector AvrStb6, which triggers Stb6-mediated immunity (Saintenac et al. 2018) that underlies GFG in the Z. tritici-wheat interaction. We previously developed a mapping population between Z. tritici isolates IP0323 and IP094269 (Goodwin et al. 2011; Wittenberg et al. 2009; Kema et al. 2002; Mirzadi Gohari et al. 2015) that we saturated here with Diversity Array Technology (DarTseq) markers (Tables S2, 3 and 4, Figure S1) and mapped a putative avirulence effector gene on the tip of chromosome 5 (Table 1, Figure 1). Public RNAseq data (Rudd et al. 2015) were used to predict a single gene candidate (four exons; Figure S2, 3 and 4), which was highly expressed in planta, encoding a small secreted protein (82 amino acids [aa], 12 cysteines, mature size 63 aa; Figure 1). Deletion in the avirulent strain IP0323 resulted in compatibility on cv. Shafir that carries Stb6, identifying the candidate as AvrStb6. Introducing AvrStb6 into the compatible strain IP094269 resulted in incompatibility on cv. Shafir, thereby demonstrating that AvrStb6 is recognized by Stb6 (Figure 1, Figure S5). Recently, AvrStb6 was also identified in a genome-wide association study and subsequent ectopic integration in a virulent $Z$. tritici strain (Zhong et al. 2017). Analyses of the IPO323/IP094269 mapping population and a 
panel of Z. tritici isolates suggests that pathogenicity on cultivars carrying Stb6 is consistent with two amino acid changes in the AvrStb6 protein (Table S4, Figure S6). 


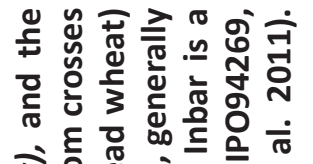

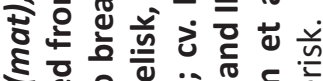

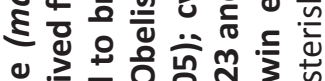

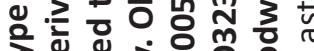

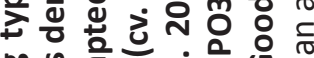

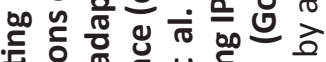
突

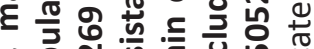
응 는 는

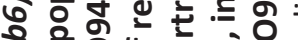

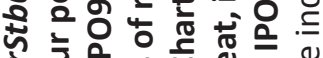

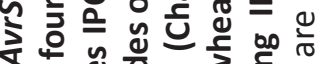

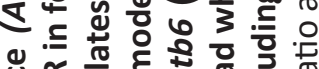

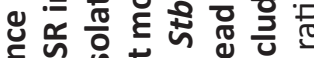

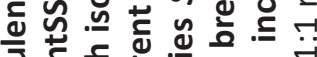

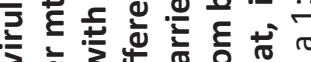

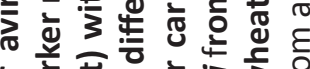

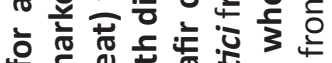

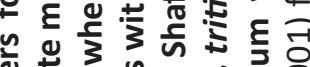

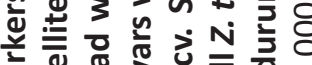
变 政 E

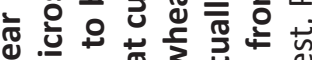
ब

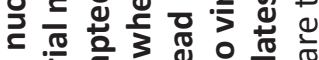

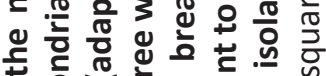

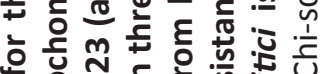
응

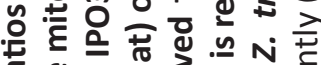

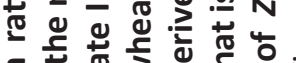

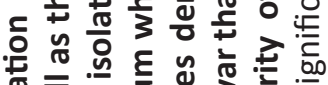

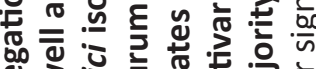

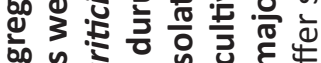

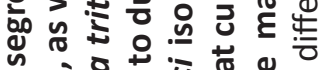
40

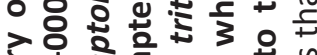
政

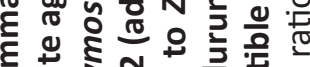

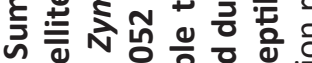
证

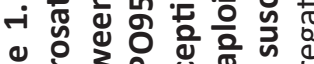

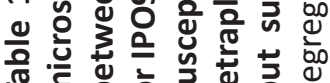

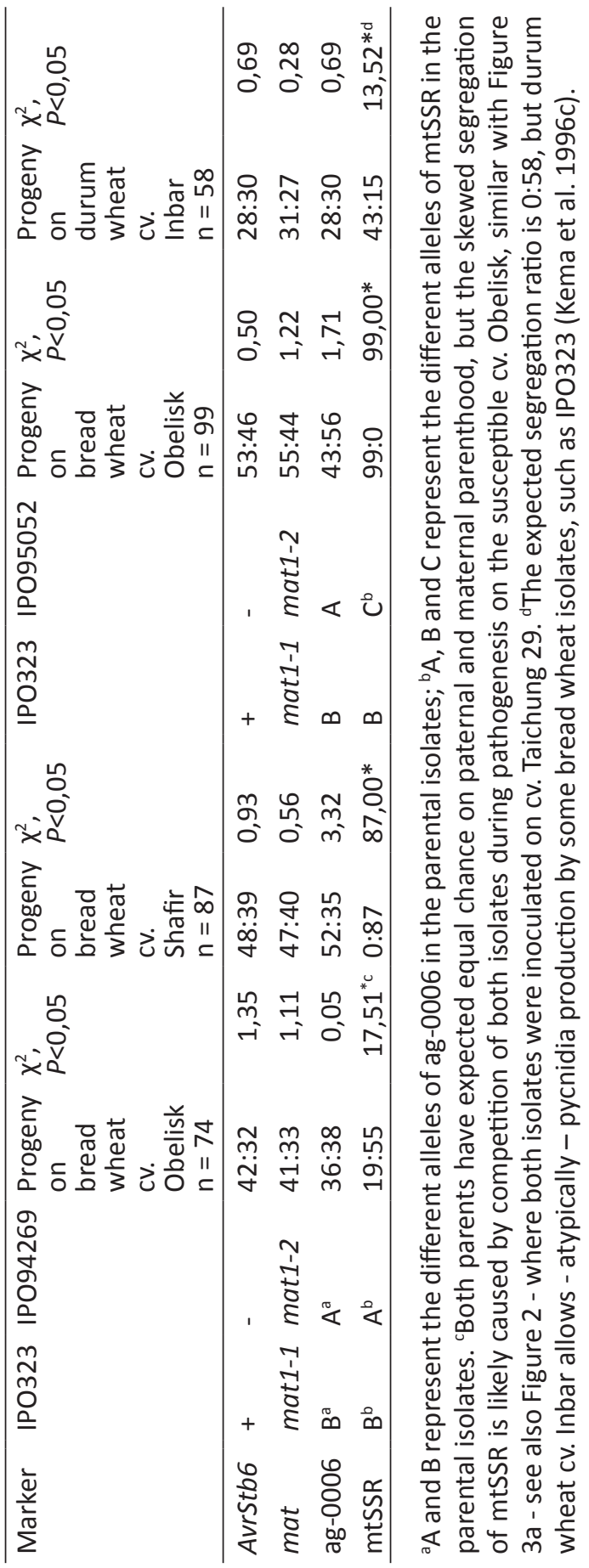




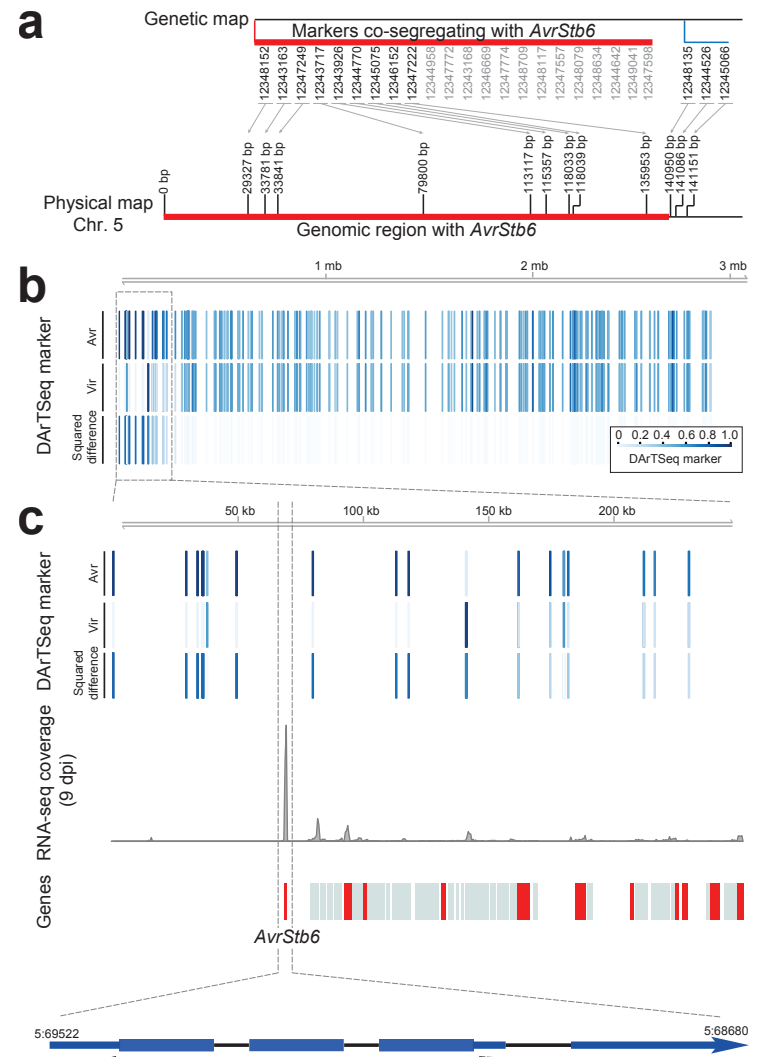

MRSILOGUAFALAVGVOARVSGGGIGDLCKAGDSCCNYPGTDCFQDGQYPRCHTACGHFOFGFCHDGKOCNGQVILGCGCV

$$
\text { d }
$$

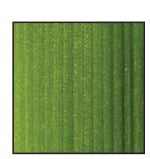

Mock

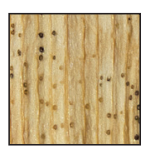

\#14

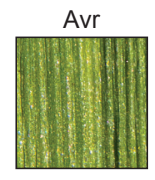

IPO323

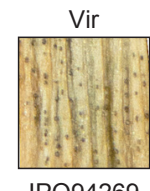

IPO94269

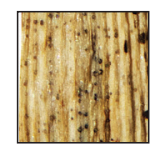

\#33

IPO323 $\Delta$ AvrStb6
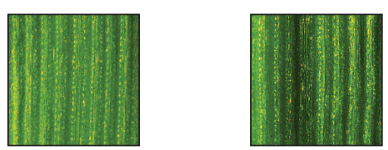

\#2

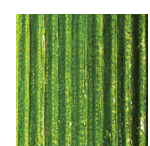

IPO323::hyg E
Figure 1. Cloning of AvrStb6 in Zymoseptoria tritici isolate IPO323. (a) Genetic and physical maps of a tip of chromosome 5: cluster of 22 DArTSeq markers fully co-segregating with AvrStb6 are highlighted in red (markers with non-unique mapping in grey) and flanking markers in blue. Marker locations (bp) are indicated by arrows and the genomic region harboring AvrStb6 in red. (b) Genomic locations of DArTSeq markers on chromosome 5 are indicated by colored lines. The dashed rectangle highlights the only polymorphic region that is characterized by high squared differences of the fractions of DArTSeq markers in avirulent or virulent progeny (color-coded [scale 0-1]). (c) Magnification of the first 250 $\mathrm{kb}$ on chromosome 5 with the genomic location of the DArTSeq markers. The positions of predicted genes and genes encoding secreted proteins are indicated by grey and red bars, respectively. RNAseq reads (Rudd et al. 2015) indicate a single, highly expressed gene, designated AvrStb6, encoding a secreted, cysteine-rich effector protein. The blue line indicates exon-intron structure with coding regions shown with extended line width. Amino acids in green indicate the predicted signal peptide, whereas cysteines are shown in red. (d) Phenotyping of Z. tritici on cv. Sharif (carrying Stb6). Top: mock and wt Z. tritici isolates IPO323 and IP094269. Middle: independent knock-outs of AvrStb6 in IPO323 are virulent. Bottom: two independent Introductions of AvrStb6 in virulent strain IPO94269 (\#1 and \#2) are avirulent. A strain (IPO323::hyg E) with an ectopic integration of the deletion construct in IPO323 is similar to the wt. 
Z. tritici isolates are crossed in planta, which is similar to sex in nature, to generate segregating populations (Goodwin et al. 2011; Hunter et al. 1999; Kema et al. 1996a; Shaw et al. 1989; Wittenberg et al. 2009; Brading et al. 2002; Kema et al. 2002; Kema et al. 2000; Mirzadi Gohari et al. 2015). Apart from demonstrating a GFG interaction between wheat and $Z$. tritici, we observed unexpected sexual reproduction between IPO323 and IP094269 on CV. Shafir, despite the presence of AvrStb6 in the avirulent parent IPO323 (Table 1). Sexual reproduction was further confirmed by crossing IPO323 and IPO95052 on the cvs. Obelisk or Inbar, which are susceptible to IPO323 and resistant to IP095052 or vice versa, respectively (Table 1). We analysed the four progenies with three nuclear markers (the avirulence gene AvrStb6; the mating type alleles mat1-1 or mat1-2; a random nuclear SSR marker) and a mitochondrial SSR marker (mt-SSR) and conclude that IPO323, despite its avirulence, undergoes sexual reproduction with isolates IP094269 or IP095052 (Table 1, Figure S7). Thus, although IPO323 cannot infect cvs. Shafir and Inbar, it completes a sexual cycle, thereby maintaining AvrStb6 in subsequent populations (Figure 2 and Figure S8). Moreover, crosses between sexually compatible Z. tritici strains never fail unless both parents are avirulent (Table S3). Notably, IPO323 is the exclusive paternal donor in the cross with the virulent isolate IP094269 on cv. Shafir, but swaps to the exclusive maternal - and virulent - donor in crosses with the avirulent isolate IPO95052 on cv. Obelisk, as shown by the mt-SSR marker that is only maternally inherited (Rudd et al. 2015) (Table 1), as well as on cv. Taichung 29 (Figure 3a). Thus, isolate IPO323 circumvents unfavorable host conditions (i.e. resistance) via sexual reproduction as male partner; a mechanism that we here call exclusive paternal parenthood (EPP, Table 1, Figure 2). Hence, we conclude that host resistance is a biotic stress factor that modulates parenthood in fungal sex. Therefore, our data challenge the common belief (Thrall et al. 2015; Brown et al. 2011) that avirulent individuals disappear from natural populations since they can neither infect nor reproduce on resistant hosts. 
a

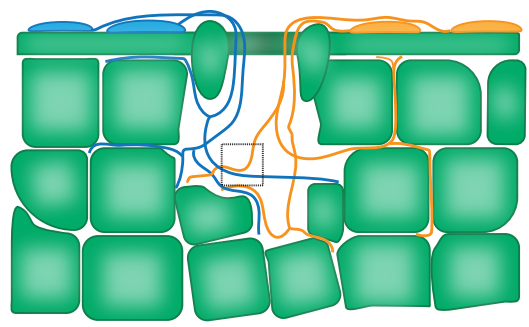

b
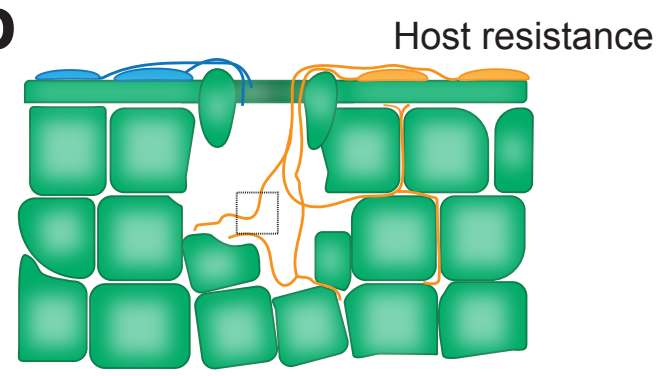

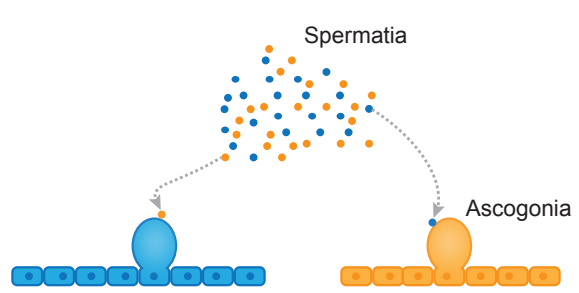

mat 1-1 mat 1-2

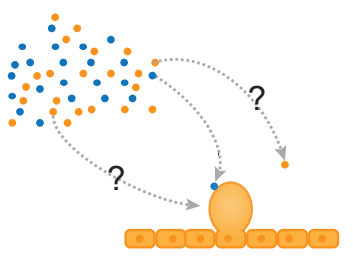

mat 1-2

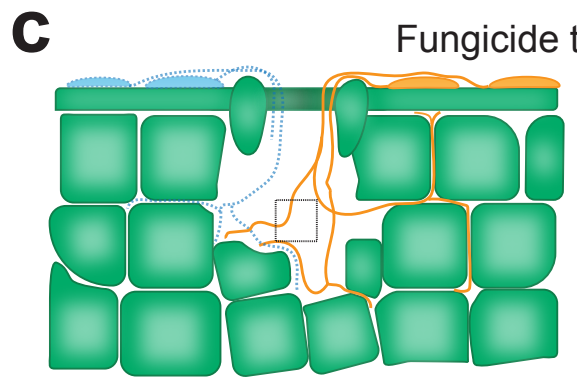

\section{treatment}

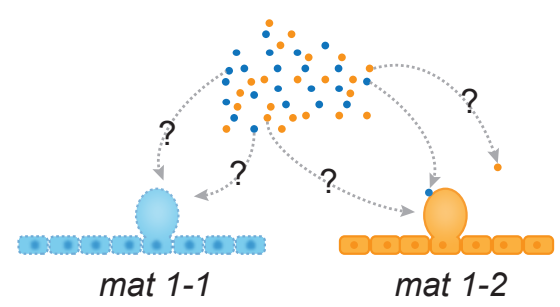

Figure 2. Sex in Zymoseptoria tritici. The fungus has a heterothallic bipolar mating system. Each strain has a unique mating type, either mat1-1 (blue) or mat1-2 (orange). When both strains infect the same host, they produce female (ascogonia) and male (microconidia or spermatia) (Annone 1984; Alexopoulos 1962) reproductive organs. Both strains have equal chances for maternal or paternal parenthood. Heterothallism defines that mat1-1 ascogonia are exclusively fertilized by mat1-2 spermatia and vice versa. (a) Optimal conditions for two pathogenic strains. (b) An avirulent strain (mat1-1, blue) encounters biotic stress on resistant wheat, despite penetration (Kema et al. 1996b). The virulent strain (mat1-2, orange) colonizes the mesophyll. Biotic stress reduces biomass of the avirulent strain, but allows the production of spermatia. Exclusive paternal parenthood (EPP) determines that ascogonia of the virulent strain are exclusively fertilized by the avirulent strain. Consequently, avirulence genes are transmitted to the progeny and distributed by airborne ascospores. (c) The sensitive isolate (mat1-1, blue dotted line) is under abiotic stress, while the resistant strain (mat1-2, orange solid line) colonizes the host after strobilurin application. Sensitive strains are shown during colonization or just after penetration for strobilurin applications under 
field conditions are preventive/curative. Abiotic stress reduces biomass of the sensitive strain, but allows the production of spermatia. EPP determines that mating is exclusively accomplished by fertilizing the ascogonia of the resistant strain. Consequently, the entire progeny caries the cytb gene with the G143A mutation (fungicide resistance), which is maternally transferred and further disseminated by airborne ascospores.

a Individual inoculations

Co-inoculations
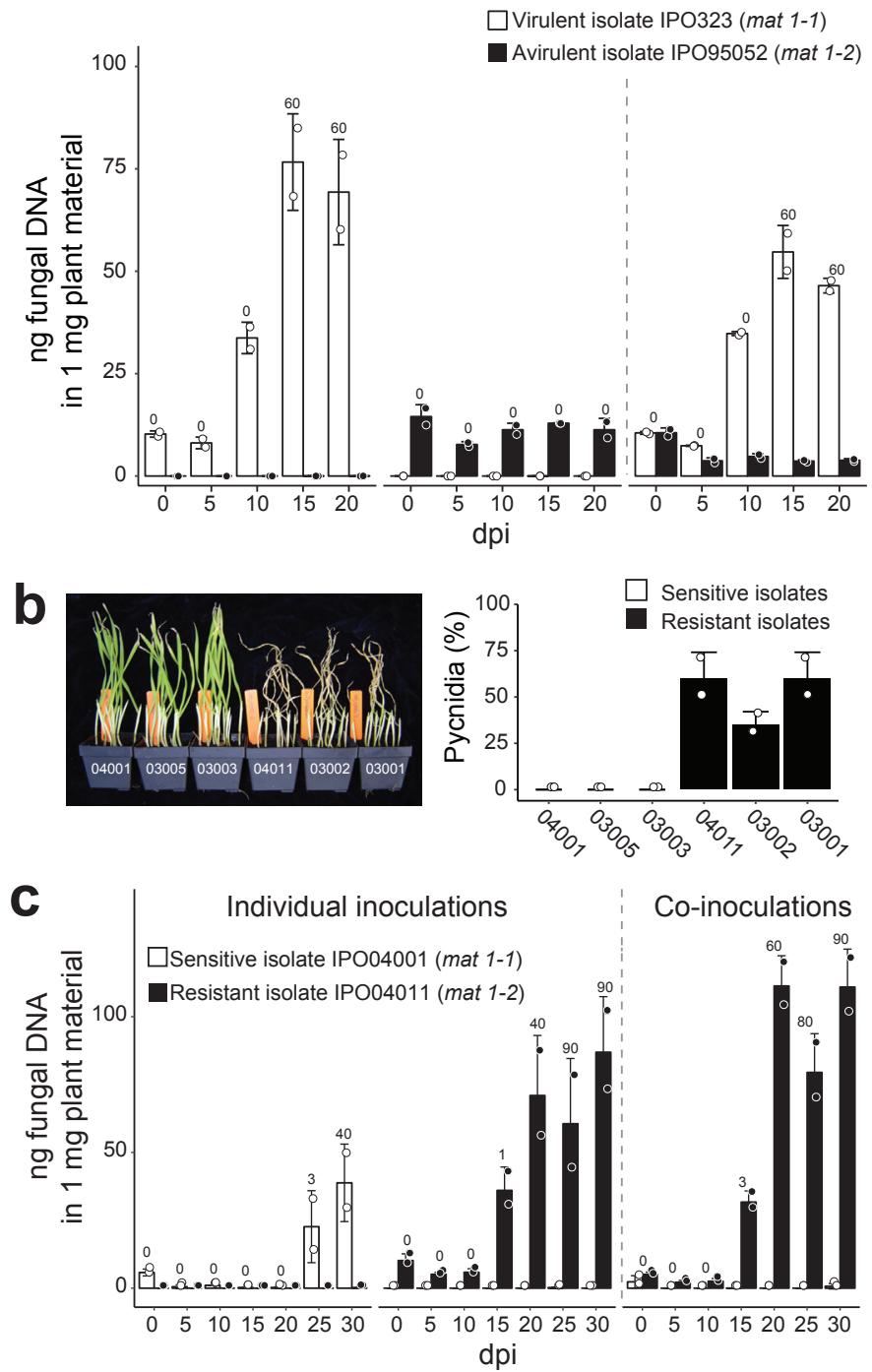

Figure 3. Inoculation and mating/competition assays with Zymoseptoria tritici. (a) Quantitative biomass detection of isolates IPO323 (virulent) and IP095052 (avirulent) 
and their co-inoculations on the bread wheat cv. Taichung 29 at 0, 5, 10, 15, and $20 \mathrm{dpi}$ (bars: average of two independent experiments; whiskers show standard deviations). Percent leaf area covered by pycnidia at each time point shown as numbers over bars. (b) Fungicide sensitivity screen at $20 \mathrm{dpi}$. Plants of wheat cv. Taichung 29 treated (48h prior to inoculation) with the full recommended rate of the strobilurin Amistar ${ }^{\circledR}$ (active ingredient [ai] azoxystrobin) and inoculated with the sensitive isolates 04001,03005 or 03003 and with the resistant isolates 04011, 03002 or 03001 (right panel; percent pycnidia based on visual observations, average of two independent experiments, whiskers show standard deviations). This resulted in significantly different disease severities between the sensitive and resistant strains (both panels). (c) Quantitative biomass detection of $Z$. tritici on cv. Taichung 29 after preventative treatment ( $48 \mathrm{~h}$ prior to inoculation) with the full recommended field rate of Amistar $^{\circledR}$ at $0,5,10,15,20,25$, and 30 dpi (three independent crossing experiments for the phenomenon; bars are averages of two technical replicates, whiskers show standard deviations). Plants were inoculated with the sensitive isolate IPO04001, the resistant isolate IPO04011, and both (co-inoculations). Percent leaf area covered by pycnidia at each time point is shown as numbers over each bar.

To generalize these observations, we considered fungicides as abiotic stress factors for Z. tritici and hypothesized that they result in EPP of sensitive strains. We used the strobilurin fungicide Amistar ${ }^{\circledR}$ and resistance as the maternally inherited marker (Figure 3, Figure S9). Six Z. tritici field isolates originating from Germany and The Netherlands with equal pathogenicity, opposite mating types and contrasting fungicide resistance were crossed in three sets (Table 2, Table S5, Figure 3, Figure S10). We produced 42 progenies under various concentrations of Amistar $^{\circledR}$ (Table 2) and the percentage of resistant ascospores was determined through either visual observation $(9,025$ ascospores) or by PCR assays on 2,100 progeny isolates (50 per cross) (Table 2). Despite the use of fungicides, we confirmed sexual reproduction for all crosses (Table 2, Figure S11). Sensitive strains were outcompeted in each crossing assay (Figure 3, Figure S10). Under normal and double azoxystrobin concentrations all progenies were entirel fixed for resistance in one generation (Table 2). Thus, Amistar ${ }^{\circledR}$ applications direct resistant and sensitive isolates into maternal and paternal parenthood, respectively, leading to a rapidly increasing frequency of resistance alleles in the generated progenies. In conclusion, we observed that biotic and abiotic stresses may hamper or restrict host colonization, but cannot preclude sexual reproduction as male gametes (spermatia) presumably survive (a)biotic stresses (Figure 2).

Table 2. Non-Mendelian inheritance of resistance to azoxystrobin in ascospore progeny populations of Zymoseptoria tritici. a, The percentage of strobilurinresistant progeny was determined by monitoring the germination of 9,025 ascospores, originating from 21 in planta crosses between strobilurin resistant and sensitive $Z$. tritici isolates on seedlings of wheat cv. Taichung 29 that were preventively treated with six doses of Amistar $^{\circledR}$ and then discharged onto water agar amended with 1 ppm (MIC value) Amistar ${ }^{\circledR} \square$ normalized to germination frequencies of 15,975 ascospores from the 
same crosses that were discharged to unamended water agar. $\mathbf{b}$, The percentage of strobilurin resistant progeny determined by a strobilurin sensitivity PCR screen in $42 \mathrm{Z}$. tritici progenies.

\begin{tabular}{|c|c|c|c|c|c|c|}
\hline \multirow{2}{*}{$\begin{array}{l}\text { a } \\
\text { Rate }\end{array}$} & \multicolumn{2}{|c|}{$\begin{array}{l}\text { IPO03001 [R] x IPO03003 } \\
\text { [S] }\end{array}$} & \multicolumn{2}{|c|}{$\begin{array}{l}\text { IPO03002 [R] x IPO03005 } \\
{[S]}\end{array}$} & \multicolumn{2}{|c|}{$\begin{array}{l}\text { IPO04011 [R] x IPO04001 } \\
{[S]}\end{array}$} \\
\hline & Total & $\%$ germination & Total & $\%$ germination & Total & $\%$ germination \\
\hline 0 & 630 & 93 & 856 & 15 & 2108 & 49 \\
\hline $1 / 32$ & 85 & 89 & 390 & 48 & 709 & 64 \\
\hline $1 / 16$ & 188 & 92 & 183 & 78 & 556 & 100 \\
\hline $1 / 8$ & 191 & 76 & 135 & 88 & 607 & 100 \\
\hline $1 / 4$ & 237 & 100 & 166 & 88 & 336 & 99 \\
\hline $1 / 2$ & 496 & 99 & 105 & 93 & 349 & 100 \\
\hline $\begin{array}{l}\text { full } \\
\text { dose }\end{array}$ & 186 & 96 & NA & NA & 512 & 100 \\
\hline Total & \multicolumn{2}{|l|}{2013} & \multicolumn{2}{|l|}{1835} & \multicolumn{2}{|l|}{5177} \\
\hline \multicolumn{7}{|l|}{ b } \\
\hline \multicolumn{7}{|c|}{$\%$ resistant in PCR } \\
\hline Rate & 1st round & 2nd round & 1 st round & 2 nd round & 1st round & 2 nd round \\
\hline 0 & 100 & 100 & 0 & 33 & 100 & 38 \\
\hline $1 / 32$ & 100 & 100 & 98 & 49 & 96 & 71 \\
\hline $1 / 16$ & 100 & 100 & 100 & 100 & 65 & 98 \\
\hline $1 / 8$ & 100 & 100 & 100 & 98 & 100 & 100 \\
\hline $1 / 4$ & 100 & 100 & 96 & 98 & 100 & 100 \\
\hline $1 / 2$ & 100 & 100 & 92 & 92 & 100 & 100 \\
\hline $\begin{array}{l}\text { full } \\
\text { dose }\end{array}$ & $*$ & 100 & $*$ & 100 & $*$ & 100 \\
\hline $\begin{array}{l}2 x \\
\text { full } \\
\text { dose }\end{array}$ & $*$ & 100 & $*$ & 100 & $*$ & 100 \\
\hline
\end{tabular}


We developed a population genetic model by incorporating EPP into Leonard's seminal model of GFG coevolution of a plant-pathogen system (Leonard et al. 1969). In this model, a plant has one locus with alleles for resistance and susceptibility, and the pathogen has a corresponding locus with alleles for avirulence and virulence. The proportion of each allele in a well-mixed population is modelled over time. In real-life cases alleles often co-exist in stable or cyclic polymorphisms, however in Leonard's model the frequency of resistance and virulence alleles in the respective population only results in fixation of one of the genes - coexistence is not possible (Figure 4). Hence, Leonard's model forms the theoretical framework to identify traits whose inclusion can result in stable or cyclic polymorphisms (such as having multiple pathogen cycles per plant cycle, including a seed bank, or incorporating spatial structure) (Goodwin et al. 2011). We explore the consequences of incorporating the EPP reproduction mechanism into the Leonard model under two scenarios: firstly, when the frequency of the plant alleles is constant, as can be assumed in an agricultural system, and secondly, when the frequency of the plant is free to vary, as in a natural ecosystem. In the agricultural scenario, the frequency of virulence in the pathogen population increases slower when avirulent strains partake in sexual reproduction on resistant hosts (Figure 4). Additionally, polymorphisms (where the two alleles can coexist indefinitely) are possible, although unlikely, which is not the case without the EPP mechanism (Tellier et al. 2007). This implies that resistance in crop cultivars will erode slower, which can have significant consequences for the sustainability of disease control in crop production systems. In the natural scenario, our model (Figure 4) shows stable or cyclic polymorphisms occurring across a wide range of parameter values. We therefore showed that the presence of sex under biotic stress allows the occurrence of stable polymorphisms simply as a result of the pathogens' genetic system. Moreover, the model confirms that when fungicide sensitive strains partake in sex, the mitochondrially inherited cytb resistance allelle invades faster than any nuclear inherited fungicide resistance allelle (not shown). 

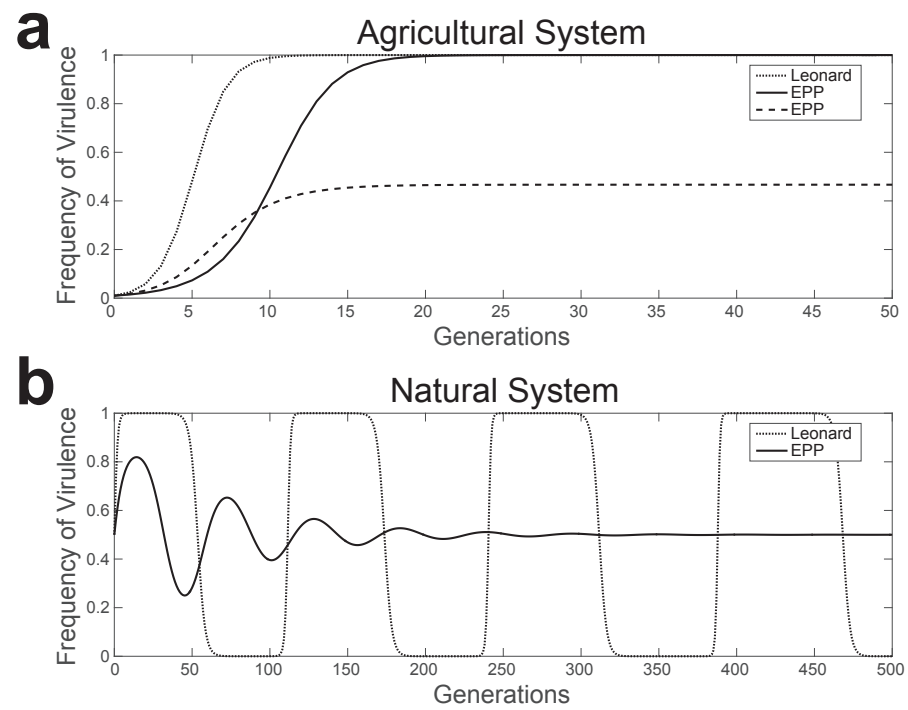

Figure 4. Comparison of the EPP model and Leonard's model in an agricultural and natural scenario. (a) In the agricultural scenario, the frequency of the resistance allele in the plant population is constant, as it is under the control of growers and not affected by selection pressures imposed by the pathogen. Comparing the EPP model and Leonard's model demonstrates the reduced rate of virulence build-up. The dashed EPP line represents a different set of parameters and demonstrates a polymorphism in the pathogen population. (b) In the natural scenario, the resistance allele frequency is dynamic and controlled by selection pressures in the system. Comparing Leonard's model with the EPP model demonstrates the possible stability of the internal equilibrium point in the EPP model, and the instability of the corresponding internal equilibrium point in Leonard's model (see Supplementary Note for different parameter sets).

The experimental data and our theoretical model provide explanations for practical observations. Slow decline of host resistance is commonly observed in the wheat- $Z$. tritici pathosystem matching the unanticipated, but ubiquitous presence of Stb6 in many old and contemporary wheat cultivars around the world (Table S1) (Chartrain et al. 2005; Kema et al. 1997). Compared to the typical boom-and-bust cycle in the yellow rust pathogen Puccinia striiformis, resistance to septoria tritici blotch declined significantly slower over a period of 10 years in the United Kingdom (Brown et al. 2015). Strobilurin fungicides were commercially introduced in 1996 and showed initially excellent control of a wide range of plant pathogens including Z. tritici. However, in 1998 resistance appeared for powdery mildew in wheat, caused by Blumeria graminis (Sierotzki et al. 2000), in 2002 for Z. tritici, which then occurred throughout Europe one year later and presently strobilurin resistance is fixed in the vast majority of $Z$. tritici populations (Fraaije et al. 2005; Torriani et al. 2009). A similar trend for strobilurin 
resistance dynamics was observed in Pseudocercospora fijiensis, the banana Black Sigatoka fungus (Amil et al. 2007; Arango Isaza et al. 2016).

Plant disease management mostly relies on host resistance or fungicide applications (Price et al. 2015; Diaz-Trujillo et al. 2017). Therefore, our observations on fungal sex have a broad relevance for developing resistant host varieties and shaping disease control strategies. This not only applies to plant pathogens, but also to human fungal pathogens such as Aspergillus fumigatus, where sex probably also contributes to the development of new life-threatening resistance mechanisms (O'Gorman et al. 2009; Verweij et al. 2016). We conclude that fungal sex is an underestimated aspect in disease control that requires much more attention.

\section{ACKNOWLEDGEMENTS}

We acknowledge financial support from the Sixth EU Framework Program Food Quality and Safety priority, the Dutch Ministry of Agriculture, Nature and Food Quality and Bayer CropScience, the Monsanto's Beachell-Borlaug International Scholars Program, the L'Oréal-UNESCO-For Women in Science Fellowship, the Consejo Nacional de Ciencia y Tecnología, Mexico, the Netherlands Organization for Scientific Research and the Dioraphte Foundation. We thank Odette Mendes, Ineke de Vries and Casper van Schaik for general support and Bruce A McDonald for sharing unpublished results. 


\section{REFERENCES}

Alexopoulos, C. Introductory Mycology, 613 (John Wiley \& Sons Inc., 1962).

Amil, A.F., Heaney, S.P., Stanger, C. \& Shaw, M.W. Dynamics of Qol sensitivity in Mycosphaerella fijiensis in Costa Rica during 2000 to 2003. Phytopathology 97, 1451-1457 (2007).

Annone, J. Washington State University (1984).

Arango Isaza, R.E. et al. Combating a global threat to a clonal crop: banana black sigatoka pathogen Pseudocercospora fijiensis (synonym Mycosphaerella fijiensis) genomes reveal clues for disease control. PLoS Genetics 12, e1005876 (2016).

Barratt, R.W., Johnson, G.B. \& Ogata, W.N. Wild-type and mutant stocks of Aspergillus nidulans. Genetics 52, 233-246 (1965).

Barrett, L.G., Thrall, P.H., Burdon, J.J. \& Linde, C.C. Life history determines genetic structure and evolutionary potential of host-parasite interactions. Trends in Ecology \& Evolution 23, 678-685 (2008).

Brading, P.A., Verstappen, E.C.P., Kema, G.H.J. \& Brown, J.K.M. A gene-for-gene relationship between wheat and Mycosphaerella graminicola, the septoria tritici blotch pathogen. Phytopathology 92, 439-445 (2002).

Brown, J.K.M. \& Tellier, A. Plant-parasite coevolution: bridging the gap between genetics and ecology. Annual Review of Phytopathology 49, 345-367 (2011).

Brown, J.K.M. Durable resistance of crops to disease: a darwinian perspective. Annual Review of Phytopathology 53, 513-539 (2015).

Cha, R.S., Zarbl, H., Keohavong, P. \& Thilly, W.G. Mismatch amplification mutation assay (MAMA): application to the C-H-ras gene. Genome Research 2, 14-20 (1992).

Chartrain, L., Brading, P.A. \& Brown, J.K.M. Presence of the Stb6 gene for resistance to septoria tritici blotch (Mycosphaerella graminicola) in cultivars used in wheatbreeding programmes worldwide. Plant Pathology 54, 134-143 (2005b).

Courtois, B. et al. Genome-wde association mapping of root traits in a japonica rice panel. PLoS One 8, e78037 (2013).

Diaz-Trujillo, C. et al. A new mechanism for reduced sensitivity to demethylationinhibitor fungicides in the fungal banana black Sigatoka pathogen Pseudocercospora fijiensis. Molecular Plant Pathology. https://doi.org/10.1111/ mpp.12637 (2017).

Emanuelsson, O., Brunak, S., von Heijne, G. \& Nielsen, H. Locating proteins in the cell using TargetP, SignalP and related tools. Nature Protocols 2, 953-971 (2007).

Fraaije, B.A. et al. Role of ascospores in further spread of Qol-resistant cytochrome $b$ alleles (G143A) in field populations of Mycosphaerella graminicola. Phytopathology 95, 933-941 (2005).

Goodwin, S.B. et al. Finished genome of the fungal wheat pathogen Mycosphaerella graminicola reveals dispensome structure, chromosome plasticity, and stealth pathogenesis. PLoS Genetics 7, e1002070 (2011).

Hahne, F. et al. Gviz: Plotting data and annotation information along genomic coordinates. $R$ package version 1 (2013). 
Holt, C. \& Yandell, M. MAKER2: an annotation pipeline and genome-database management tool for second-generation genome projects. BMC Bioinformatics 12 , 1-14 (2011).

Hunter, T., Coker, R.R. \& Royle, D.J. The teleomorph stage, Mycosphaerella graminicola, in epidemics of septoria tritici blotch on winter wheat in the UK. Plant Pathology 48, 51-57 (1999).

Johnson, A.D. et al. SNAP: a web-based tool for identification and annotation of proxy SNPs using HapMap. Bioinformatics 24, 2938-2939 (2008).

Kellner, R. et al. Expression profiling of the wheat pathogen Zymoseptoria tritici reveals genomic patterns of transcription and host-specific regulatory programs. Genome Biology and Evolution 6, 1353-1365 (2014).

Kema, G.H.J. \& van Silfhout, C.H. Genetic variation for virulence and resistance in the wheat-Mycosphaerella graminicola pathosystem III. Comparative seedling and adult plant experiments. Phytopathology 87, 266-272 (1997).

Kema, G.H.J. et al. A combined amplified fragment length polymorphism and randomly amplified polymorphism DNA genetic linkage map of Mycosphaerella graminicola, the septoria tritici leaf blotch pathogen of wheat. Genetics 161, 1497-1505 (2002).

Kema, G.H.J. et al. Genetic variation for virulence and resistance in the wheatMycosphaerella graminicola pathosystem. I. Interactions between pathogen isolates and host cultivars. Phytopathology 86, 200-212 (1996b).

Kema, G.H.J., Sayoud, R., Annone, J.G. \& Van Silfhout, C.H. Genetic variation for virulence and resistance in the wheat-Mycosphaerella graminicola pathosystem. II. Analysis of interactions between pathogen isolates and host cultivars. Phytopathology 86, 213-220 (1996c).

Kema, G.H.J., Verstappen, E.C.P. \& Waalwijk, C. Avirulence in the wheat septoria tritici leaf blotch fungus Mycosphaerella graminicola is controlled by a single locus. Molecular Plant-Microbe Interactions 13, 1375-1379 (2000).

Kema, G.H.J., Verstappen, E.C.P., Todorova, M. \& Waalwijk, C. Successful crosses and molecular tetrad and progeny analyses demonstrate heterothallism in Mycosphaerella graminicola. Current Genetics 30, 251-258 (1996a).

Kema, G.H.J., Yu, D.Z., Rijkenberg, F.H.J., Shaw, M.W. \& Baayen, R.P. Histology of the pathogenesis of Mycosphaerella graminicola in wheat. Phytopathology 86, 777786 (1996).

Keon, J. et al. Transcriptional adaptation of Mycosphaerella graminicola to programmed cell death (PCD) of its susceptible wheat host. Molecular Plant-Microbe Interactions 20, 178-193 (2007).

Lee, E. et al. Web Apollo: a web-based genomic annotation editing platform. Genome Biology 14, R93-R93 (2013).

Leonard, K.J. Genetic equilibria in host-pathogen systems. Phytopathology 59, 18581863 (1969).

Linde, C.C., Zhan, J. \& McDonald, B.A. Population structure of Mycosphaerella graminicola: from lesions to continents. Phytopathology 92, 946-955 (2002). 
Lukashin, A.V. \& Borodovsky, M. GeneMark.hmm: New solutions for gene finding. Nucleic Acids Research 26, 1107-1115 (1998).

Mehrabi, R. et al. Ga and Gb proteins regulate the cyclic AMP pathway that is required for development and pathogenicity of the phytopathogen Mycosphaerella graminicola. Eukaryotic Cell 8, 1001-1013 (2009).

Mehrabi, R., Zwiers, L.H., De Waard, M.A. \& Kema, G.H.J. MgHog1 regulates dimorphism and pathogenicity in the fungal wheat pathogen Mycosphaerella graminicola. Molecular Plant-Microbe Interactions 19, 1262-1269 (2006).

Mirzadi Gohari, A. et al. Effector discovery in the fungal wheat pathogen Zymoseptoria tritici. Molecular Plant Pathology 16, 931-945 (2015).

Morgulis, A. et al. Database indexing for production MegaBLAST searches. Bioinformatics 24, 1757-1764 (2008).

O'Gorman, C.M., Fuller, H.T. \& Dyer, P.S. Discovery of a sexual cycle in the opportunistic fungal pathogen Aspergillus fumigatus. Nature 457, 471-474 (2009).

Palma-Guerrero, J. et al. Comparative transcriptomic analyses of Zymoseptoria tritici strains show complex lifestyle transitions and intraspecific variability in transcription profiles. Molecular Plant Pathology 17, 845-859 (2016).

Peterson, P.D., Leonard, K.J., Roelfs, A.P. \& Sutton, T.B. Effect of barberry eradication on changes in populations of Puccinia graminis in Minnesota. Plant Disease 89, 935940 (2005).

Price, C.L., Parker, J.E., Warrilow, A.G.S., Kelly, D.E. \& Kelly, S.L. Azole fungicides - understanding resistance mechanisms in agricultural fungal pathogens. Pest Management Science 71, 1054-1058 (2015).

Rudd, J.J. et al. Transcriptome and metabolite profiling of the infection cycle of Zymoseptoria tritici on wheat reveals a biphasic interaction with plant immunity involving differential pathogen chromosomal contributions and a variation on the hemibiotrophic lifestyle definition. Plant Physiology 167, 1158-1185 (2015).

Saintenac, C. et al. An evolutionary conserved pattern-recognition receptor like protein controls gene-for-gene resistance to a fungal pathogen in wheat. Nature Genetics 50, 368-374.

Sambrook, J. \& Russell, D.W. Purification of nucleic acids by extraction with phenol:chloroform. Cold Spring Harbor Protocols 2006, pdb.prot4455 (2006).

Schmittgen, T.D. \& Livak, K.J. Analyzing real-time PCR data by the comparative CT method. 3, 1101-1108 (2008).

Seidl, M.F. \& Thomma, B.P.H.J. Sex or no sex: Evolutionary adaptation occurs regardless. Bioessays 36, 335-345 (2014).

Shaw, M.W. \& Royle, D.J. Airborne inoculum as a major source of Septoria-Tritici (Mycosphaerella graminicola) infections in winter-wheat crops in the UK. Plant Pathology 38, 35-43 (1989).

Shaw, M.W. \& Royle, D.J. Factors determining the severity of epidemics of Mycosphaerella graminicola (Septoria tritici) on winter-wheat in the UK. Plant Pathology 42, 882899 (1993). 
Sierotzki, H., Wullschleger, J. \& Gisi, U. Point mutation in cytochrome $b$ gene conferring resistance to strobilurin fungicides in Erysiphe graminis f. sp tritici field isolates. Pesticide Biochemistry and Physiology 68, 107-112 (2000).

Stanke, M., Steinkamp, R., Waack, S. \& Morgenstern, B. AUGUSTUS: a web server for gene finding in eukaryotes. Nucleic Acids Research 32, W309-W312 (2004).

Tellier, A. \& Brown, J.K.M. Polymorphism in multilocus host-parasite coevolutionary interactions. Genetics 177, 1777-1790 (2007).

Thrall, P.H., Barrett, L.G., Dodds, P.N. \& Burdon, J.J. Epidemiological and evolutionary outcomes in gene-for-gene and matching allele models. Frontiers in Plant Science 6, 1084 (2015).

Torriani, S.F.F., Brunner, P.C., McDonald, B.A. \& Sierotzki, H. Qol resistance emerged independently at least 4 times in European populations of Mycosphaerella graminicola. Pest Management Science 65, 155-162 (2009).

Trapnell, C. et al. Transcript assembly and quantification by RNA-Seq reveals unannotated transcripts and isoform switching during cell differentiation. Nature Biotechnology 28, 511-515 (2010).

Trapnell, C., Pachter, L. \& Salzberg, S.L. TopHat: discovering splice junctions with RNASeq. Bioinformatics 25, 1105-1111 (2009).

Van Ooijen. JoinMap ${ }^{\circledR}$, Software for the calculation of genetic linkage maps in experimental populations. Kyazma B.V., Wageningen, Netherlands (2006).

Verweij, P.E., Chowdhary, A., Melchers, W.J.G. \& Meis, J.F. Azole resistance in Aspergillus fumigatus: can we retain the clinical use of mold-active antifungal azoles? Clinical Infectious Diseases 62, 362-368 (2016).

Waalwijk, C., Mendes, O., Verstappen, E.C.P., de Waard, M.A. \& Kema, G.H.J. Isolation and characterization of the mating-type idiomorphs from the wheat septoria leaf blotch fungus Mycosphaerella graminicola. Fungal Genetics and Biology 35, 277286 (2002).

Ware, S.B. PhD thesis, Wageningen University, The Netherlands (2006).

Wittenberg, A.H.J. et al. Meiosis drives extraordinary genome plasticity in the haploid fungal plant pathogen Mycosphaerella graminicola. PLoS One 4, e5863 (2009).

Zadoks, J.C., Chang, T.T. \& Konzak, C.F. A decimal code for the growth stages of cereals. Weed Research 14, 415-421 (1974).

Zdobnov, E.M. \& Apweiler, R. InterProScan--an integration platform for the signaturerecognition methods in InterPro. Bioinformatics 17, 847-848 (2001).

Zhong, Z. et al. A small secreted protein in Zymoseptoria tritici is responsible for avirulence on wheat cultivars carrying the Stb6 resistance gene. New Phytologist 214, 619-631 (2017).

Zwiers, L.H. \& De Waard, M.A. Efficient Agrobacterium tumefaciens-mediated gene disruption in the phytopathogen Mycosphaerella graminicola. Current Genetics 39, 388-393 (2001). 


\section{SUPPLEMENTARY INFORMATION}

Core

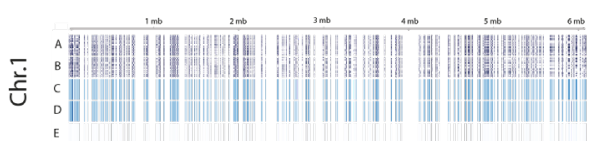

บุ $\mathrm{A}$ B

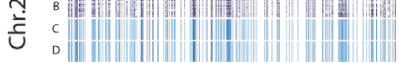

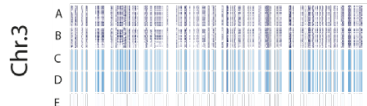

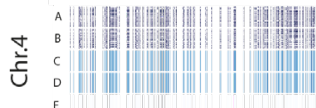

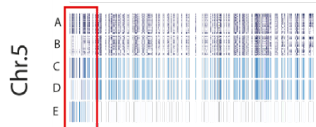

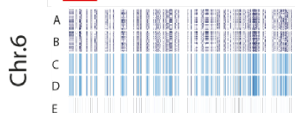

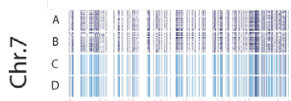

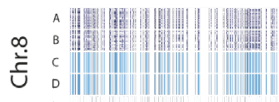

Dispensable

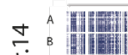

妾 $c$ ||| ||

A Hin

$\stackrel{\sim}{\sim}$ B

竎 $c$ c

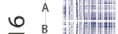

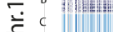

D \|\|\|\|\|\|$\|$

.

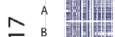

Еे $c$

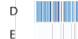

$\infty{ }_{\text {B }}^{\text {A }}$

C

टे

D

O ${ }_{B}^{A}$

言 $c$

D ||||||||||

을 B $^{\text {B }}$

导

D $\|$

- A 影期

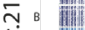

产 $c$

E

A HatI

o $B$ H

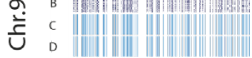

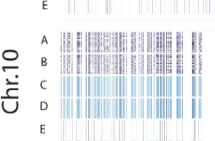

A DArTSeq markers for avirulent isolates

B DArTSeq markers for virulent isolates

C fraction of DArTSeq markers for avirulent isolates

D fraction of DArTSeq markers for virulent isolates

E squared difference

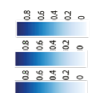

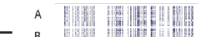

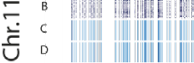

ธ

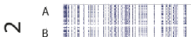

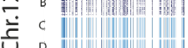

D ||||||||||||||||||||||||||

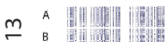

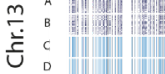


Figure S1. Genome positions of the mapped DArT markers.

Genome positions for individual DArT markers that are present (blue bars) or absent in the avirulent (A) or virulent (B) progeny (282 Z. tritici isolates) is displayed using the core chromosomes (Chr. 1-13; left) and the dispensable chromosomes (Chr.14-21; right) of $Z$. tritici IPO323 as a reference. The values of presence (1) or absence (0) of individual DArT markers was summarized for all avirulent (C) or virulent (D) progeny. This was calculated by taking the fraction of DArT marker present in the avirulent or virulent progeny (range between 0 and 1). The squared difference between the fractions of DArT markers present in the avirulent or virulent progeny is calculated, identifying a single polymorphic region in the genome of $Z$. tritici located on the tip of chromosome 5.

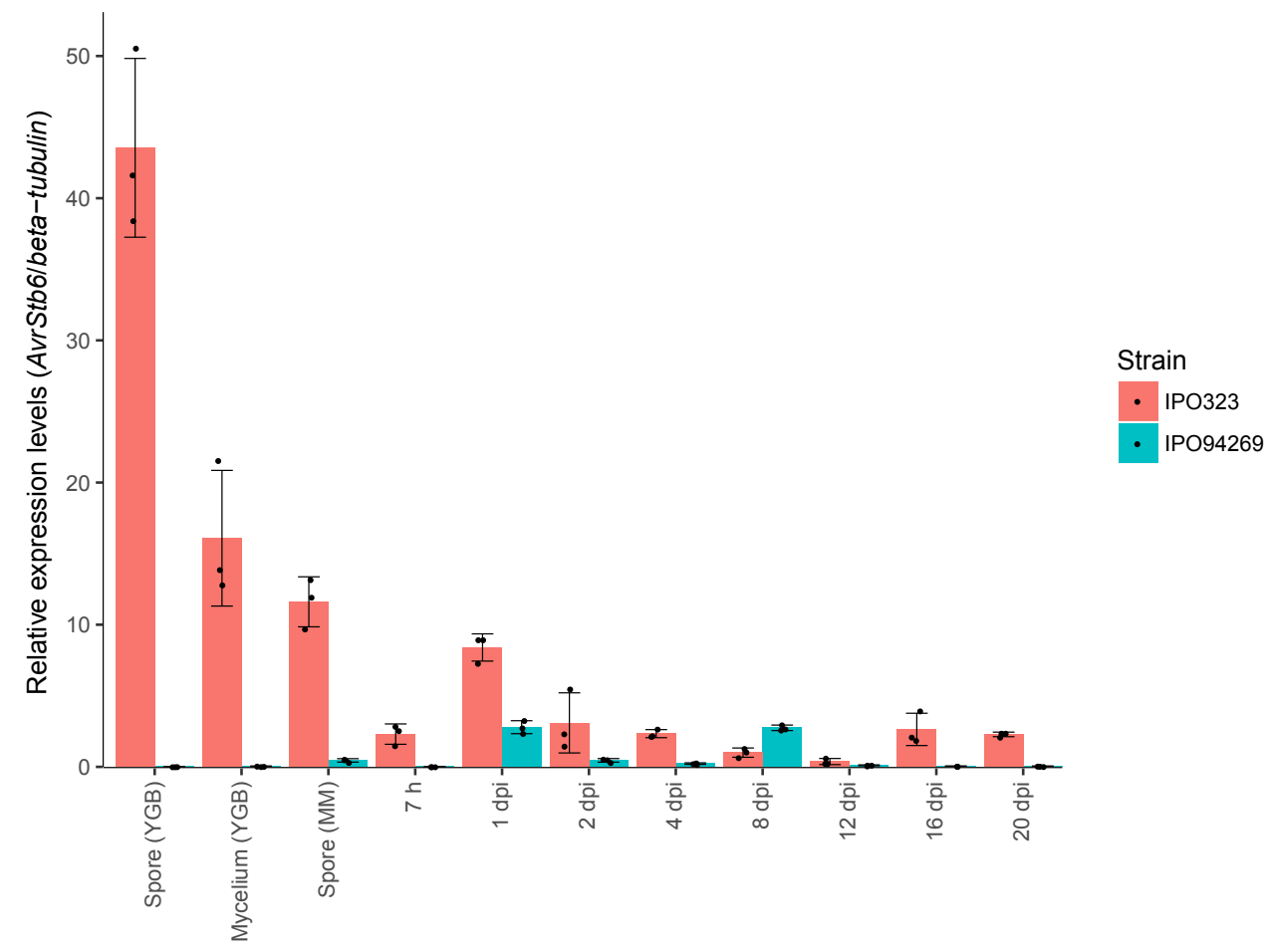

Figure S2. In vitro and in planta expression of AvrStb6. For in vitro conditions expression was profiled in blastospores in YGB and MM and in mycelium. For in planta expression, leaves of cv. Shafir were inoculated with the WT strains (IPO323 and IPO94269) and harvested 7 hour, 1, 2, 4, 8, 12, 16 and 20 days post-inoculation (dpi). Data were normalized with the constitutively expressed $Z$. tritici $\beta$-tubulin gene. 


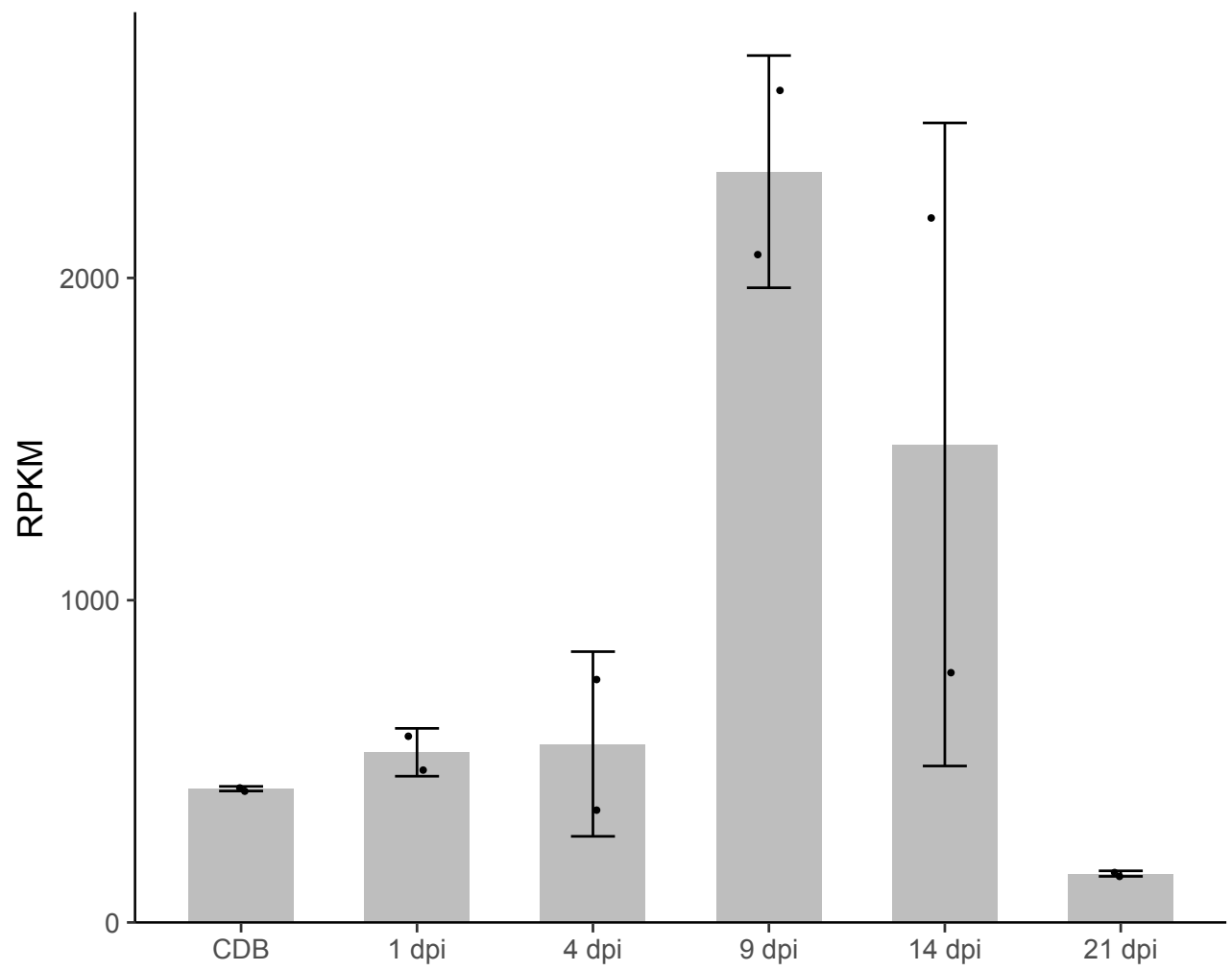

Figure S3. Expression of AvrStb6 as measured in an RNAseq experiment using various in vitro and in planta conditions (Rudd et al. 2015). 


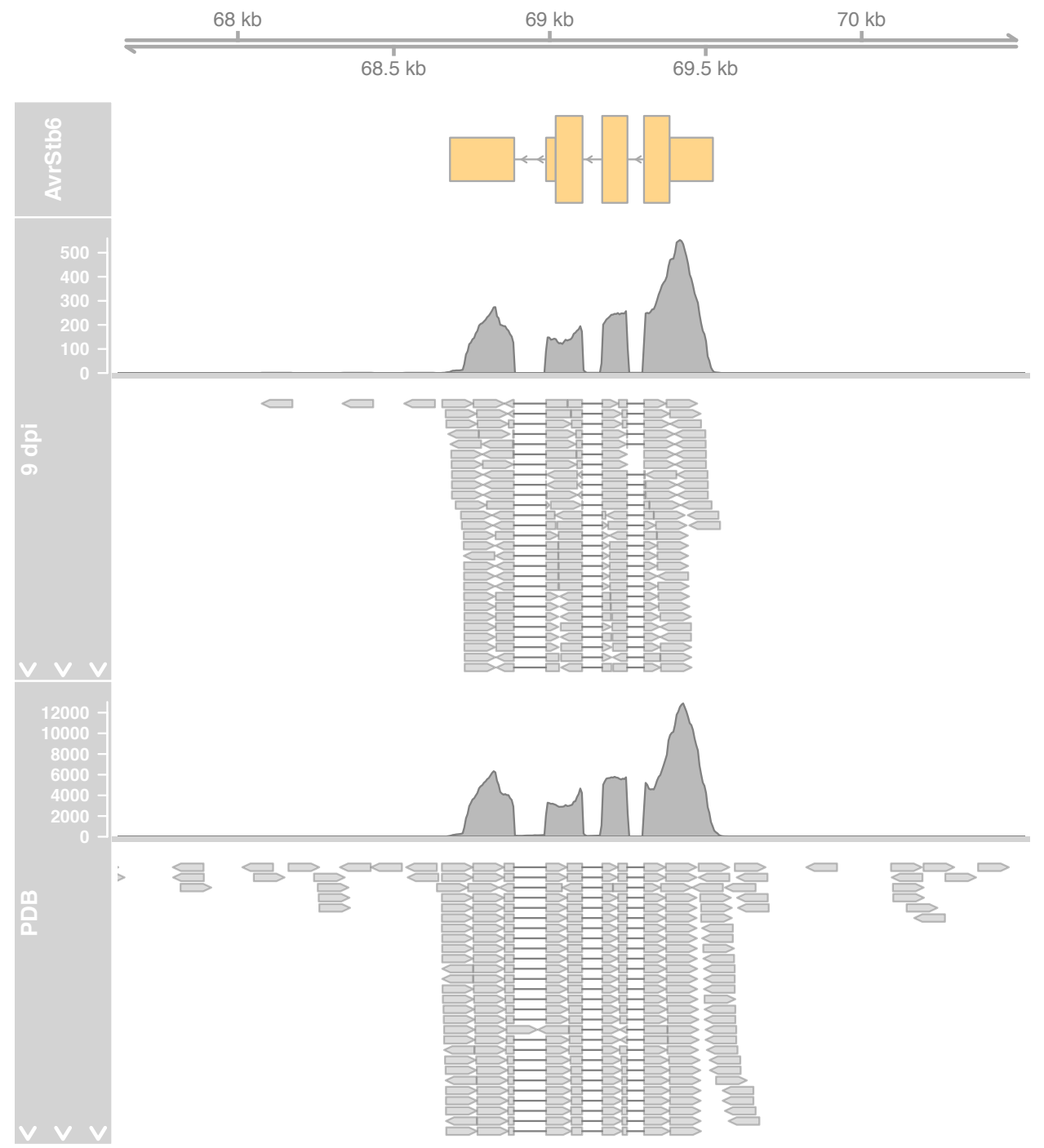

Figure S4. Genomic location of AvrStb6 on chromosome 5. The gene model (exon-intron structure) of AvrStb6 is displayed. Mapping of RNA sequencing reads to the reference genome of $Z$. tritici IPO323 is shown as a coverage and as a read alignment track. The transcriptomic data was derived from in planta (9 dpi) and in vitro (PDB) condition, respectively. 


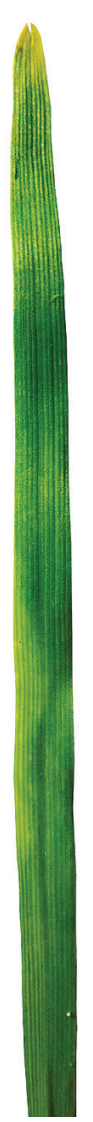

Mock
IPO323

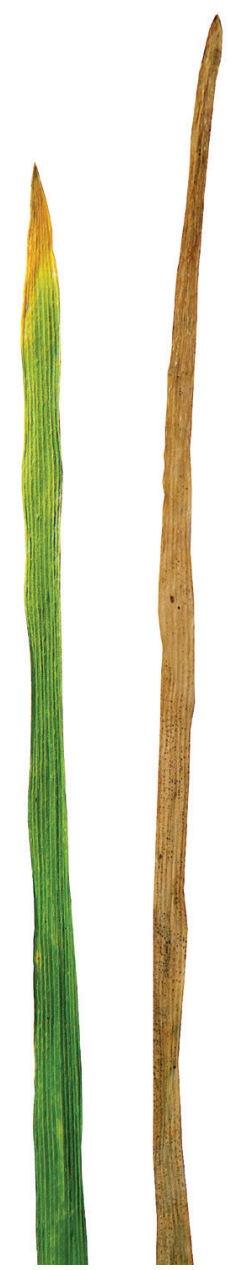

IP094269

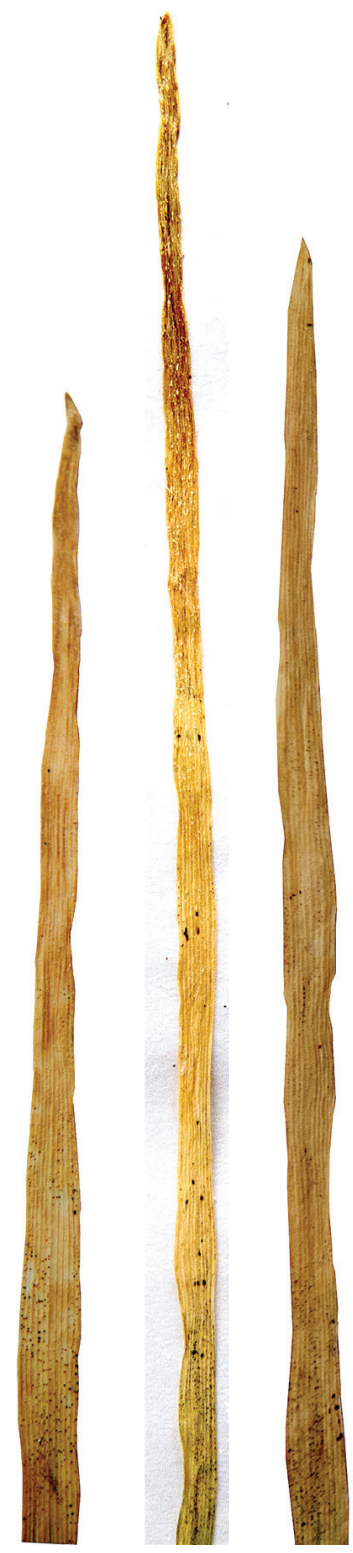

\#14

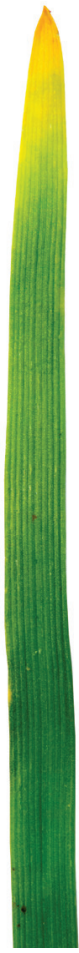

\#33

IPO323 $\triangle$ AvrStb6

Figure S5. The effect of Zymoseptoria tritici AvrStb6 deletion on disease development in the wheat cv. Shafir (Stb6). Primary leaves were inoculated with Z. tritici IPO323 and IPO94269 (WTs), all disruptants (IPO323 $\triangle$ AvrStb6\#14-19-33 and IP094269 $\Delta$ AvrStb6\#1-2) along with the ectopic strain IPO323EAvrStb6\#2. Experiments were triplicated and photographs were taken at 20 days post-inoculation. 


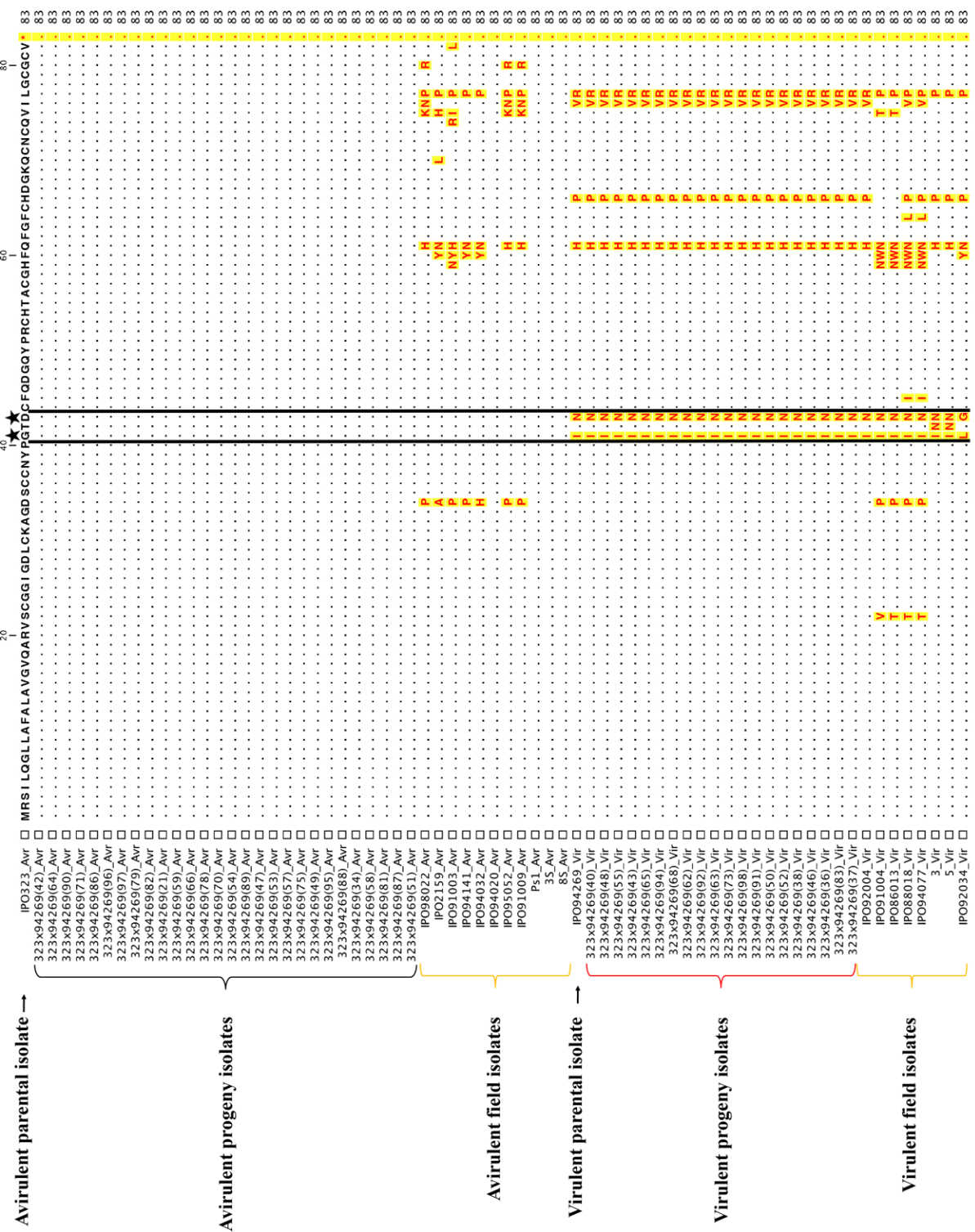

Figure S6. Protein alignment highlighting amino acid differences between virulent and avirulent Zymoseptoria tritici wild type isolates and IPO323/IP094269 progeny isolates. In the alignment amino acid substitutions are indicated, identical amino acids are denoted with "-". 
Crossed on cv. Obelisk

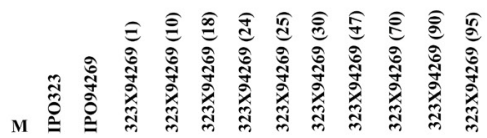

1

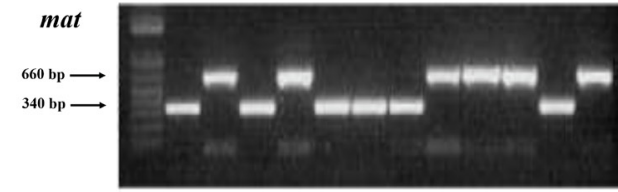

2

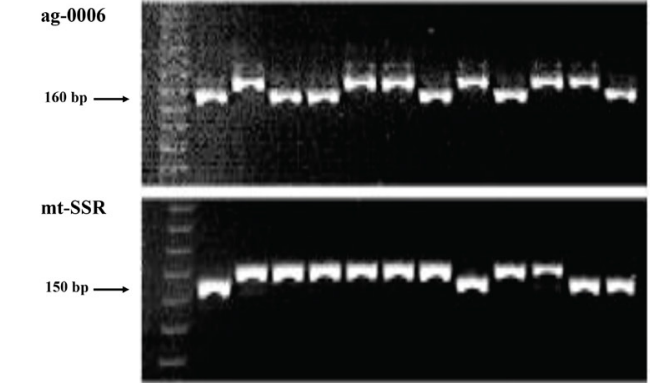

Crossed on cv. Shafir
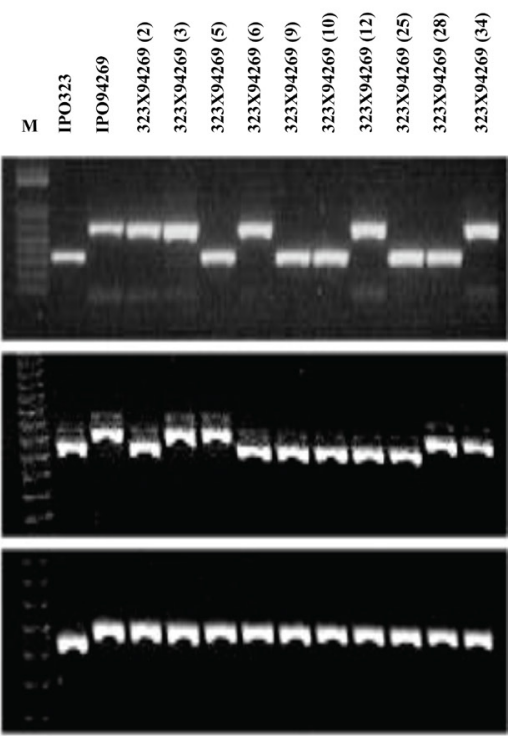

Figure S7. Examples of segregating markers in populations that were derived from in planta Zymoseptoria tritici crosses. Z. tritici isolates IPO323 and IPO94269 crossed on the bread wheat cvs. Obelisk (left) and Shafir (right). 1, mat. Upper band = mat 1-1, lower band = mat 1-2. 2, ag-0006. 3, mt-SSR (see also Table 1). 


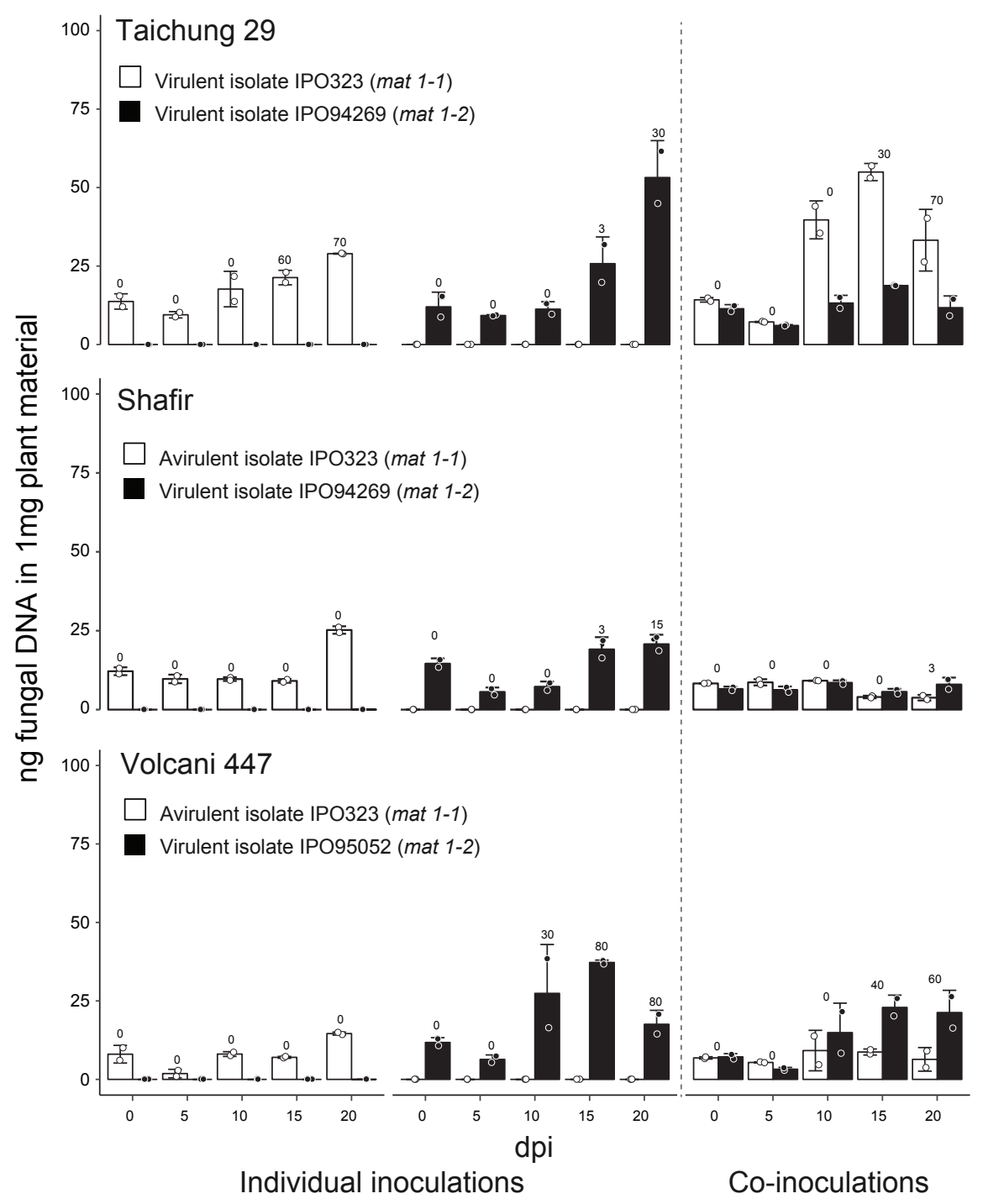

Figure S8. Quantitative fungal biomass detection of Zymoseptoria tritici isolates IPO3233 and IPO94269 on bread wheat cvs. Taichung29 and Shafir, and isolates IPO323 and IPO95052 on durum wheat cv. Volcani 447 at 0, 5, 10, 15, and 20 dpi (bars; average of two independent experiments; whiskers indicate standard deviations) and percent leaf area covered by pycnidia at each time point (numbers above each bar). 


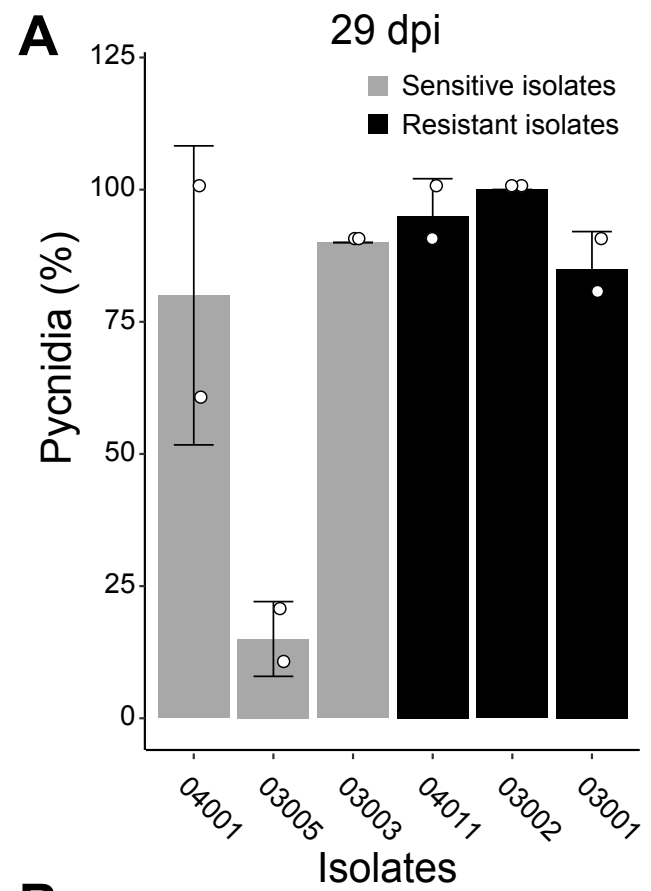

B

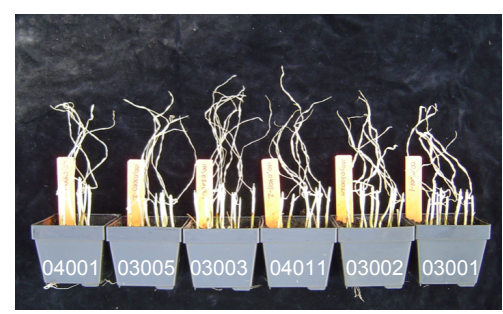

Figure S9. Responses of seedlings of wheat cv. Taichung 29 at $20 \mathrm{dpi}$ (left panels) and 29 dpi (right panels) after inoculation with resistant $(04001,03005$ and 03003$)$ or sensitive (04011, 03002 and 03001) isolates of Zymoseptoria tritici after a pre-treatment (48h prior to inoculation) with the full recommended rate of Amistarä. A) Percent pycnidia based on visual observations. B) Overall view of seedlings. 


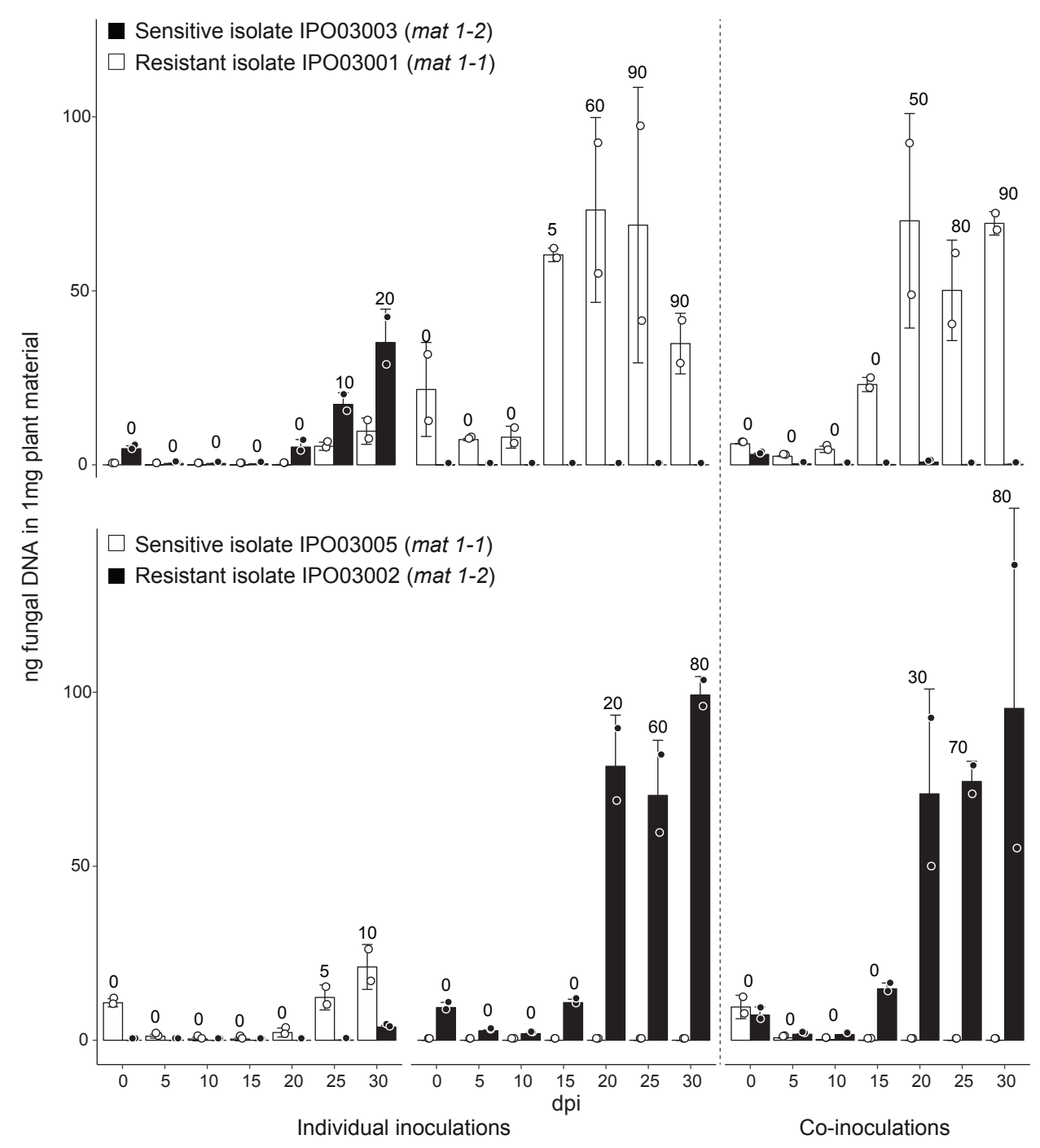

Figure S10. Fungal biomasses and percent pycnidia of parental isolates of Zymoseptoria tritici inoculated individually and in mixtures on cv. Taichung 29 after preventative treatment (48 $\mathrm{h}$ prior to inoculation) with the full recommended field rate of azoxystrobin at $\mathbf{0}, \mathbf{5}, \mathbf{1 0}, \mathbf{1 5}, \mathbf{2 0}, \mathbf{2 5}$, and $30 \mathrm{dpi}$. Left to right: A, IPO03003 (sensitive), IPO03001 (resistant), and mixture of IPO03001 and IPO03003. B, IPO03005 (sensitive), IPO03002 (resistant), and mixture of IPO03002 and IPO03005. Pycnidial percentages based on visual observations are shown above each bar. 


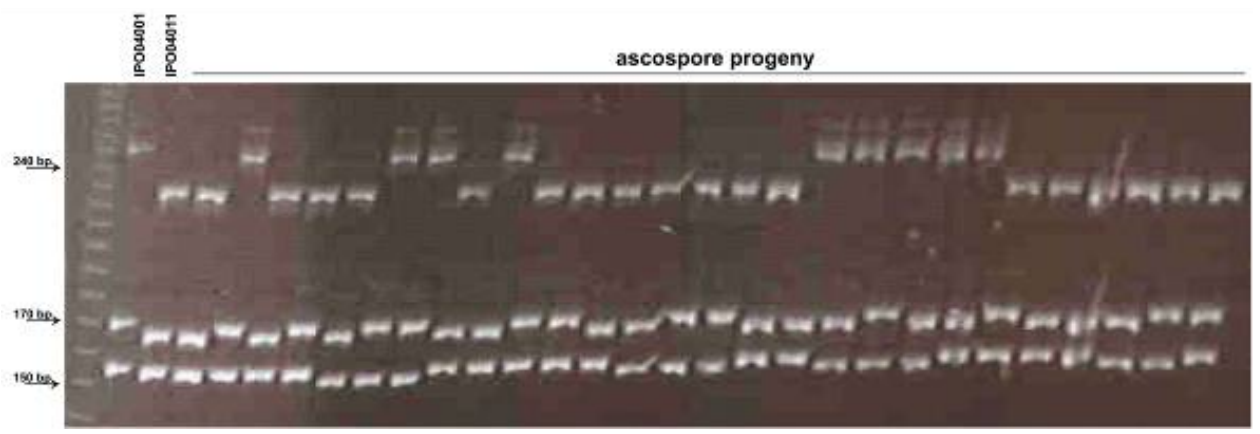

Figure S11. Example of SSR genotyping of progeny from crosses between Zymoseptoria tritici strains with opposite azoxystrobin phenotypes (resistant and sensitive). Isolates IP004001 (sensitive) and IP004011 (resistant) generated off spring on wheat seedlings preventatively treated with Amistar $^{\mathrm{TM}}$ in doses ranging from $0-200 \%$. Multi-plexed PCRs using the differentiating SSR marker primer sets ag-0003, tcc-0006 and tcc-0008 revealed recombinant SSR profiles in progeny.
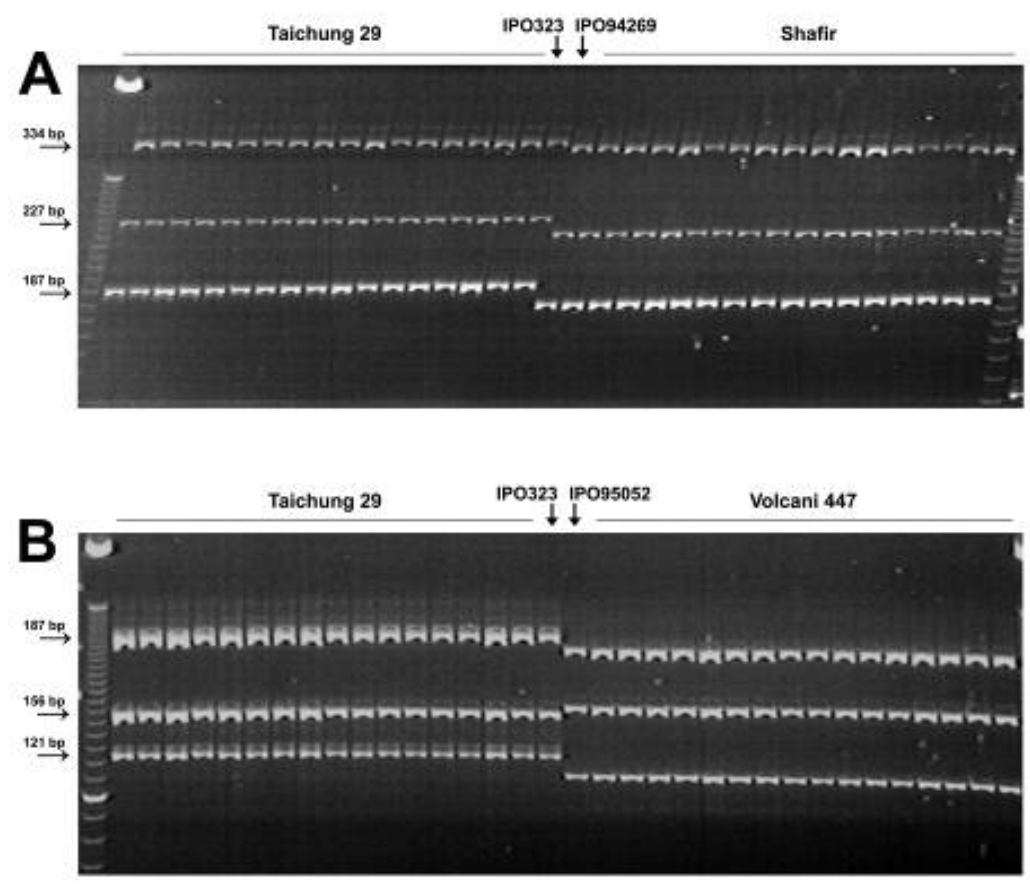

Figure S12. SSR genoyping of re-isolations of asexual pycnidial isolates from wheat leaves that were co-inoculated with Zymoseptoria tritici isolates. A) From mixtures of IPO323 and IPO94269 on cvs. Taichung 29 and Shafir using SSR markers (from top to bottom) ac-0001, 
ggc-0001, and caa-0002. B) From mixtures of IPO323 and IPO95052 on cvs. Taichung 29 and Volcani 447 using SSR markers (from top to bottom) ag-0003, ag-0006, and ac-0007.

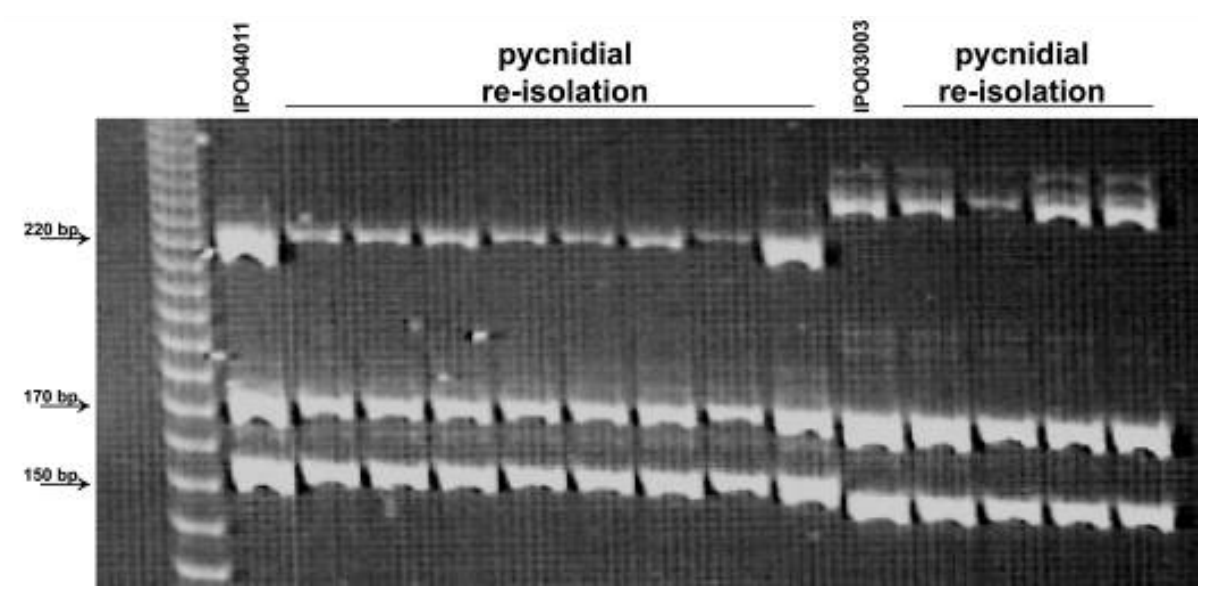

Figure S13. SSR genotyping of pycnidial isolates of Zymoseptoria tritici recovered from wheat seedlings preventatively treated with Amistar ${ }^{\mathrm{TM}}$ at half and full doses. All SSR patterns are clonal like the sensitive pycnidial isolates IPO04001 or IP003003. Multi-plexed PCRs using the differentiating SSR marker primer sets ag-0003, tcc-0006 and tcc-0008 revealed no recombinant SSR patterns in recovered pycnidial isolates. 


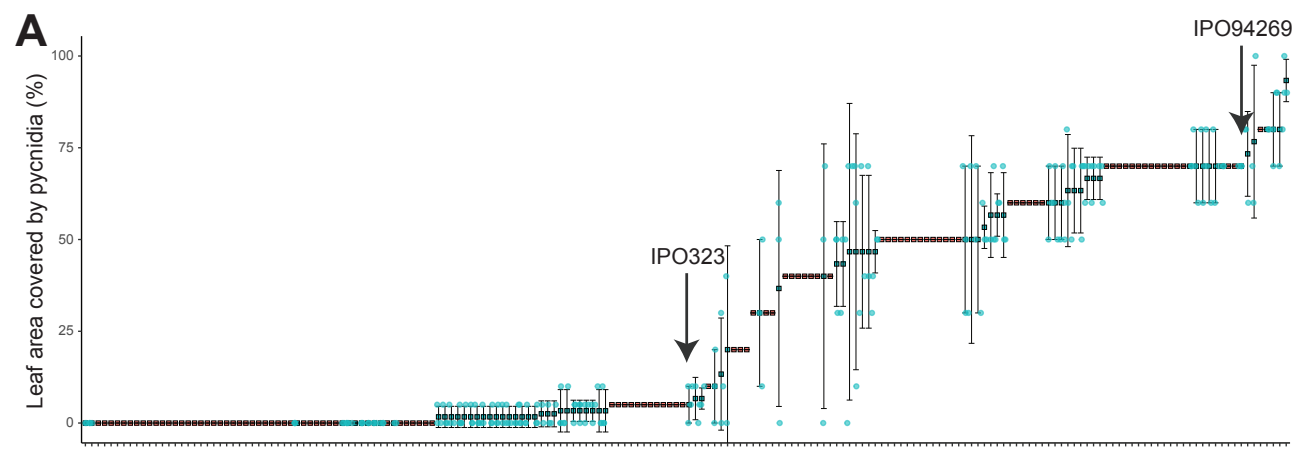

IPO323/IP094269 progeny

B

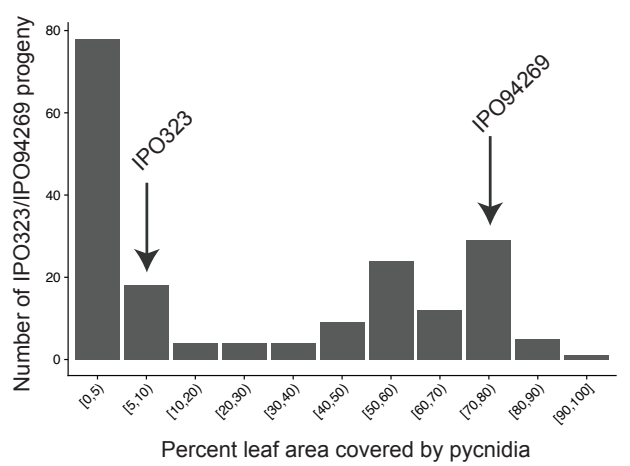

C

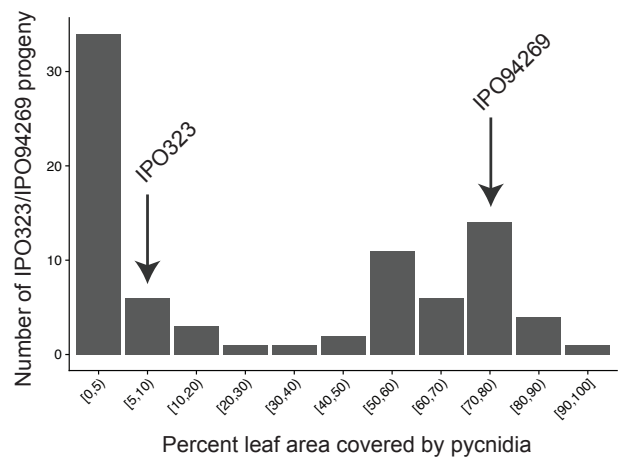

Figure S14. Segregation of the F1 IPO323/IP094269 progeny isolates for pycnidia development on cv. Shafir carrying the Stb6 resistance gene at 21 days post-inoculation. (A) Average leaf area covered by pycnidia for each IPO323/IP094269 progeny isolates and their parental isolates, is shown. Individual isolates were ordered along the $x$-axis based on their average leaf area covered by pycnidia. Isolates for which more than one up to three independent experiments have been performed are highlighted in green (whiskers indicate standard deviations), while isolates used in a single experiment are shown in red. Distributions of pycnidia development (median leaf area covered) for (B) all isolates and (C) isolates with $>1$ independent experiment are shown. Arrows indicate average parental pycnidia development on cv. Shafir.

Figure S15. (right) Graphical genotyping of DArTseq markers and (a)virulence to 'Shafir'. By sorting the progeny isolates from the F1-population from IPO323 x IPO94260 according to the positions of recombination events on chromosome 5 of Zymoseptoria tritici, the avirulence gene AvrStb6 could be positioned between the blue flanking markers as shown on the picture. The isolates are haploid, and therefore have either the maternal locus from the avirulent parent or the paternal locus from the virulent parent. Red colour indicates loci inherited from the avirulent parent IPO323, whereas loci inherited from the virulent parent IP094269 are displayed in green. Grey dashes indicates missing values. The yellow markers fully co-segregated with the (a)virulence. We only display the isolates that showed recombination near the (a)virulence locus. 


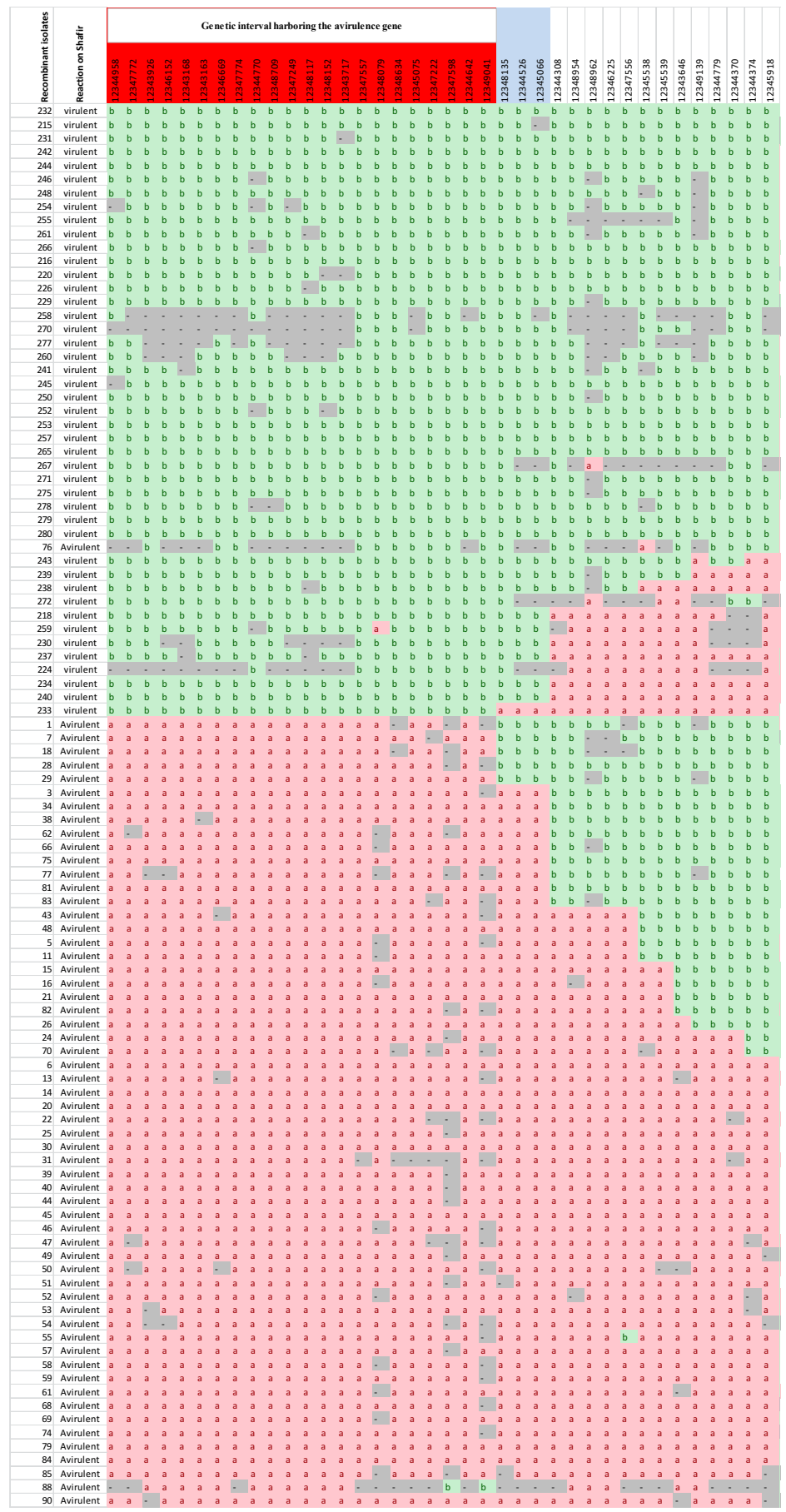




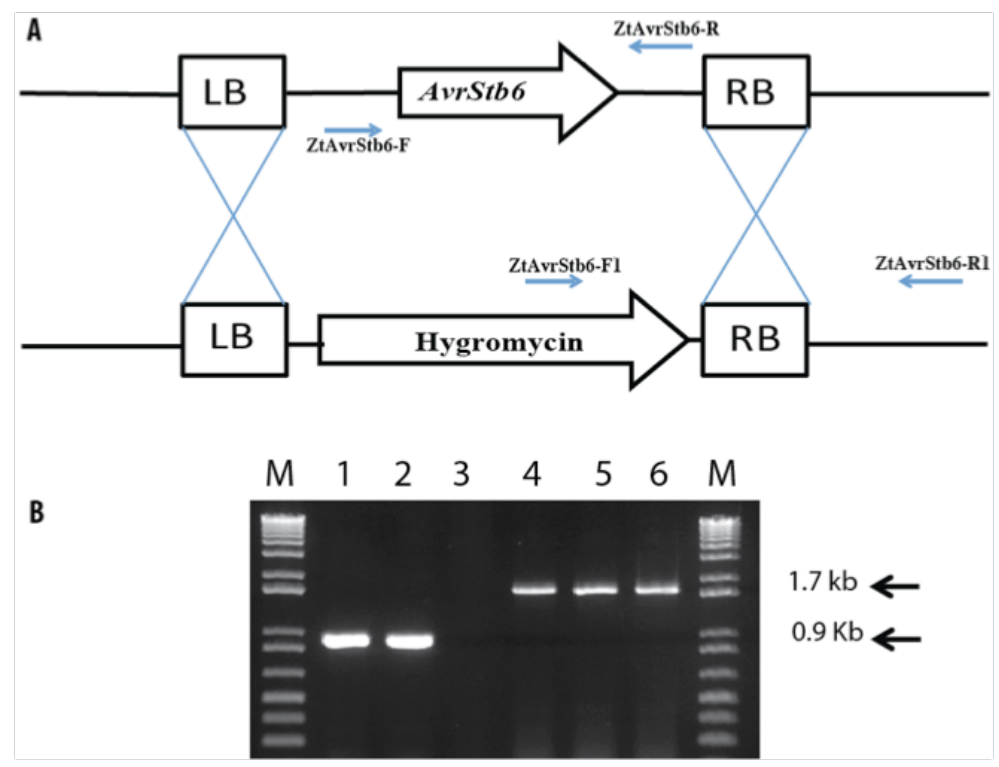

Figure S16. Replacement strategy for AvrStb6 in Zymoseptoria tritici. A) Diagram showing the replacement by the hygromycin phosphotransferase $(h p h)$ resistance cassette through homologous recombination. The dotted line depicts the flanking regions used for homologous recombination. B) Identification of replacement mutants by PCR; Lane M, 1-kbplus ladder. The gel shows the correct amplification (900 bp), using primers ZtAvrStb6-F and ZtAvrStb6-R, of AvrStb6 in IPO323 (Lane 1) and the ectopic strain IPO323EAvrStb6\#2 (Lane 2), but no amplification in the mutant strain IPO323 $\triangle$ AvrStb6\#33 (Lane 3). Amplification with primers ZtAvrStb6-F1 and ZtAvrStb6-R1, which are located in the middle of the $h p h$ gene and downstream of AvrStb6, respectively, result in a $1.7 \mathrm{~kb}$ amplicon in the three independent replacement mutants IPO323 $\Delta Z$ ZtStb6\#14, IPO323 $\Delta Z t S t b 6 \# 19$ and IPO323 $\Delta Z t S t b 6 \# 33$. 

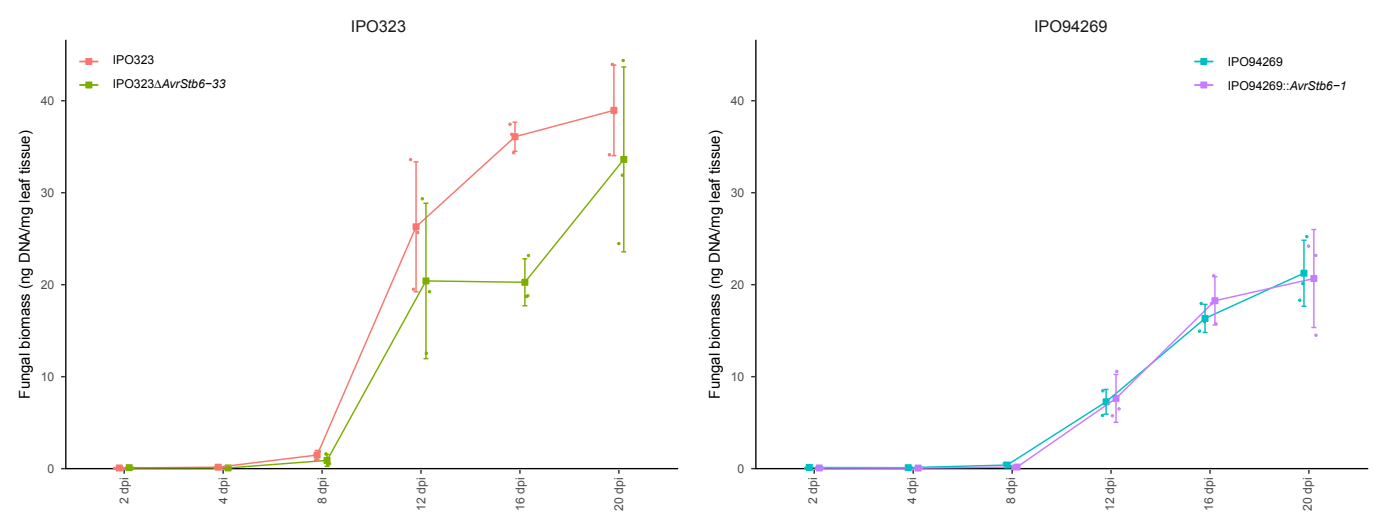

Figure S17. Fungal biomass quantifications of Zymoseptoria tritici in the susceptible wheat cv. Taichung 29 at 2, 4, 8, 12, 16 and 20 days post inoculation. A) Fungal biomass comparison of Z. tritici IPO323 (WT) and the knock-out strain IPO323 $\Delta$ AvrStb6-33, B) Fungal biomass comparison of Z. tritici IPO94269 (WT) and the AvrStb6 random integration strain IP094269::AvrStb6-1.

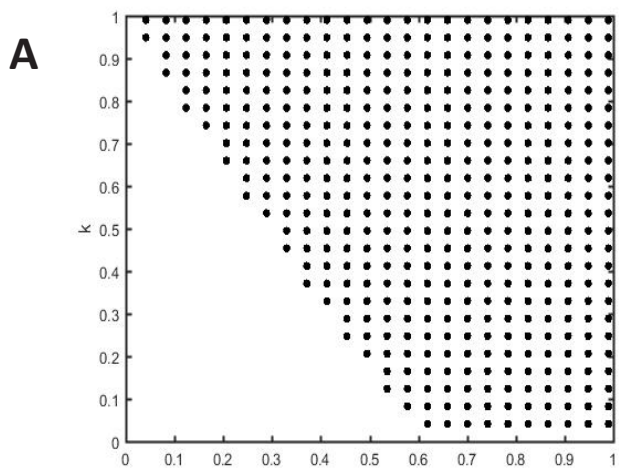

B

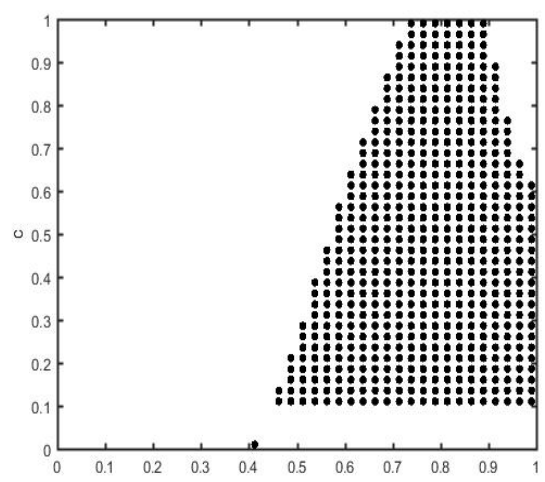

Figure S18. a. The parameter space $\boldsymbol{\sigma}$ vs $\mathbf{k}$. The dotted area of the graph shows the pair of $(\sigma, \mathrm{k})$ parameters where the internal equilibrium point is stable. In this plot, the fitness cost to the plant by being infected was 0.2 , the fitness cost to the plant of resistance was $0.1 . b$. The parameter space $\sigma_{\mathrm{vs}}{ }^{\mathrm{c}}$. The dotted area of the graph shows the pair of $(\sigma, c)$ parameters where the internal equilibrium point is stable. In this plot, the fitness cost to the pathogen of being virulent was 0.2 , the fitness cost to the plant of resistance was 0.1 . 


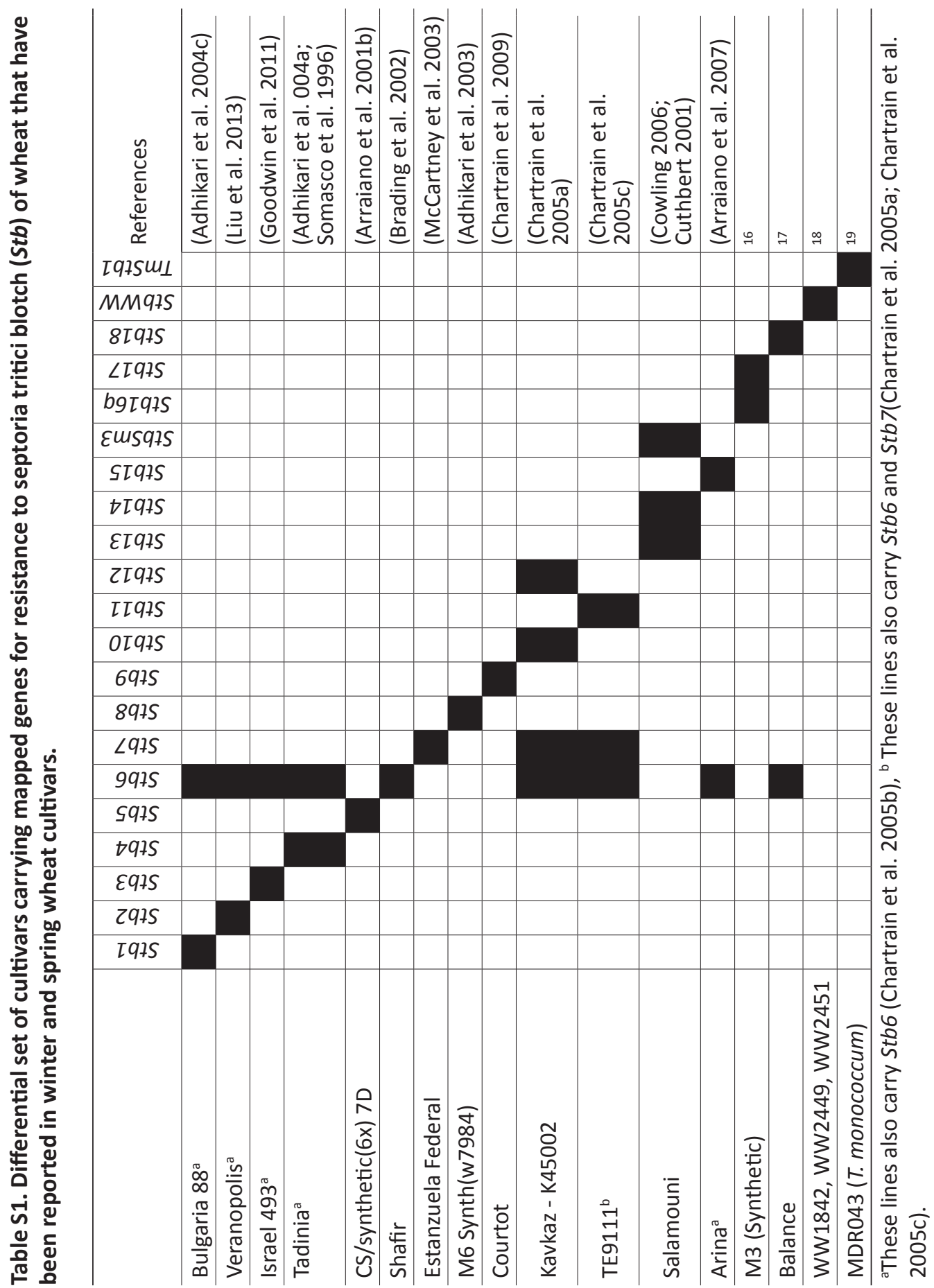


Table S2. Summary information about the Zymoseptoria tritici isolates that were used in the in planta crossing protocol on wheat cultivars with various levels of resistance.

\begin{tabular}{llllll}
\hline Isolate & Year & Origin & Host & Virulent on & Mating type \\
\hline IPO001 & unknown & Netherlands & Bread Wheat $^{1}$ & Bread Wheat & mat 1-1 \\
IP0323 & 1981 & Netherlands & Bread Wheat & Bread Wheat & mat 1-1 \\
IP087019 & 1987 & Uruguay & Bread Wheat & Bread Wheat & mat 1-2 \\
IP088004 & 1988 & Ethiopia & Durum Wheat & Durum Wheat & mat 1-2 \\
IP094269 & 1994 & Netherlands & Bread Wheat & Bread Wheat & mat 1-2 \\
IP095054 & 1995 & Algeria & Bread Wheat & Bread Wheat & mat 1-2 \\
IP095050 & 1995 & Algeria & Durum Wheat & Durum Wheat & mat 1-1 \\
IP095052 & 1995 & Algeria & Durum Wheat & Durum Wheat & mat 1-2 \\
\hline
\end{tabular}

${ }^{1}$ derived from a hexaploid derivative of a cross between bread wheat and wild emmer wheat (T. dicoccoïdes, AABB, $2 \mathrm{n}=28$ ).

Table S3. Crosses between isolates of Zymoseptoria tritici using an in planta protocol of co-inoculation of two strains with opposite mating types on both bread wheat and durum wheat cultivars and on barley. $\mathrm{V}=$ virulent; $\mathrm{A}=$ avirulent.

\begin{tabular}{lllc}
\hline Isolates Crossed $^{1}$ & \multicolumn{1}{c}{ Cultivar } & \multicolumn{1}{c}{ Species } & Progeny \\
\hline bread wheat strains & & & \\
IPO323 (V) x IPO94269 (V) & Obelisk & Bread Wheat & yes \\
IPO323 (V) x IP094269 (V) & Taichung 29 & Bread Wheat & yes \\
IPO323 (A) x IP094269 (V) & Shafir & Bread Wheat & yes \\
IPO323 (A) x IP094269 (A) & Volcani 447 & Durum Wheat & no \\
IPO323 (A) x IP094269 (A) & Topper 33 & Barley & no \\
IP0001 (V) x IP094269 (V) & Obelisk & Bread Wheat & yes \\
IP0001 (V) x IP094269 (A) & Lakhish & Bread Wheat & yes \\
IP0001 (A) x IP094269 (V) & Clement & Bread Wheat & yes \\
IPO323 (A) x IP087019 (A) & Kavkaz-K4500 & Bread Wheat & no \\
IPO323 (A) x IP088004 (A) & Veranopolis & Bread Wheat & no \\
durum wheat strains & & & \\
IPO95050 (V) x IP095052 (V) & Volcani 447 & Durum Wheat & yes \\
IPO95050 (A) x IP095052 (A) & Obelisk & Bread Wheat & no
\end{tabular}


Table S3. continued

\begin{tabular}{|c|c|c|c|}
\hline \multicolumn{4}{|c|}{ bread wheat $x$ durum wheat strains } \\
\hline IP0323 (A) x IP095052 (V) & Inbar & Durum Wheat & yes \\
\hline IPO323 (V) x IP095052 (A) & Obelisk & Bread Wheat & yes \\
\hline IP0323 (A) x IP095052 (A) & Shafir & Bread Wheat & no \\
\hline IP094269 (A) x IP095050 (V) & Inbar & Durum Wheat & yes \\
\hline IPO94269 (V) x IPO95050 (A) & Obelisk & Bread Wheat & yes \\
\hline IP095054 (A) x IP095050 (V) & Inbar & Durum Wheat & yes \\
\hline IP095054 (V) x IP095050 (A) & Obelisk & Bread Wheat & yes \\
\hline
\end{tabular}




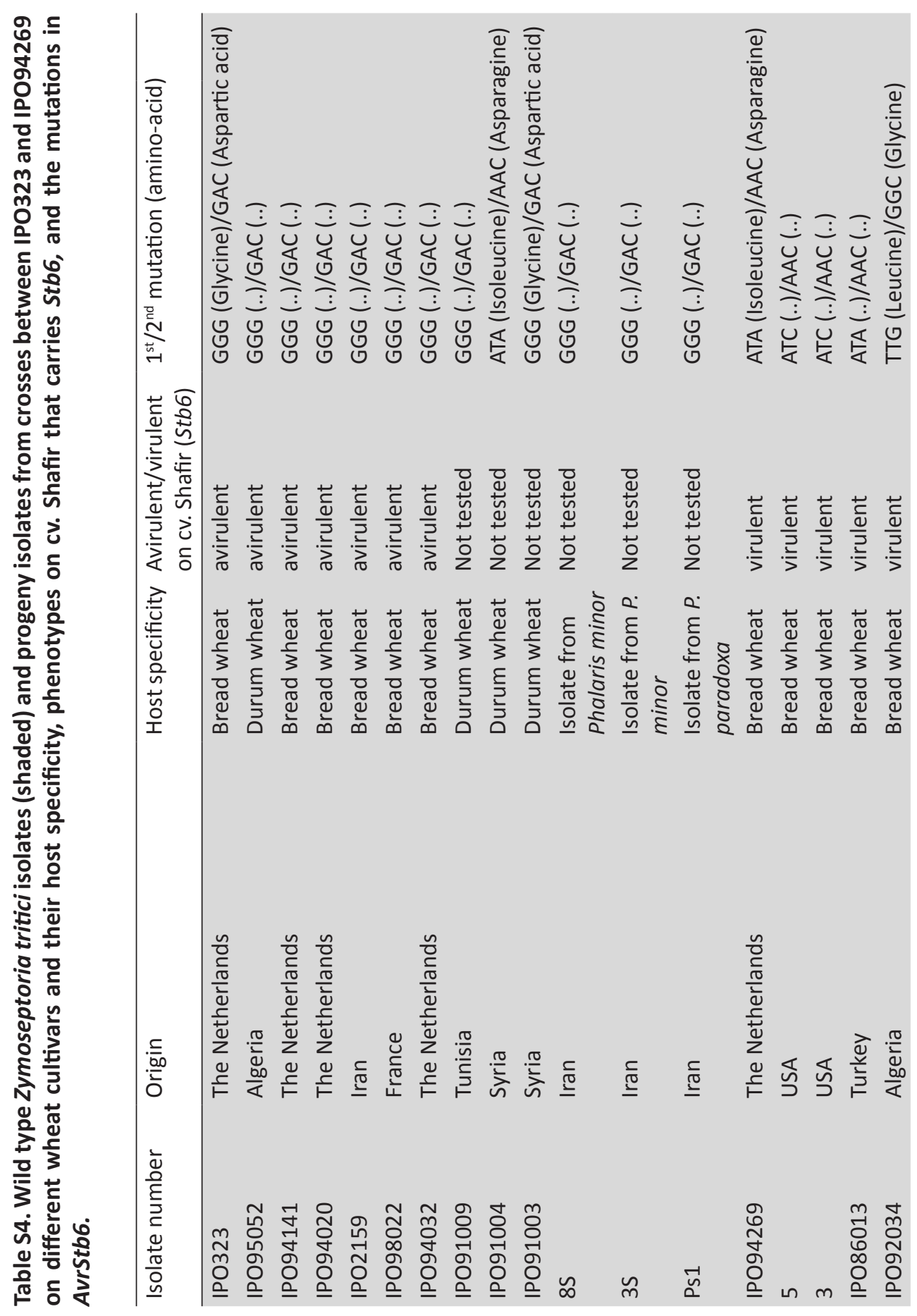




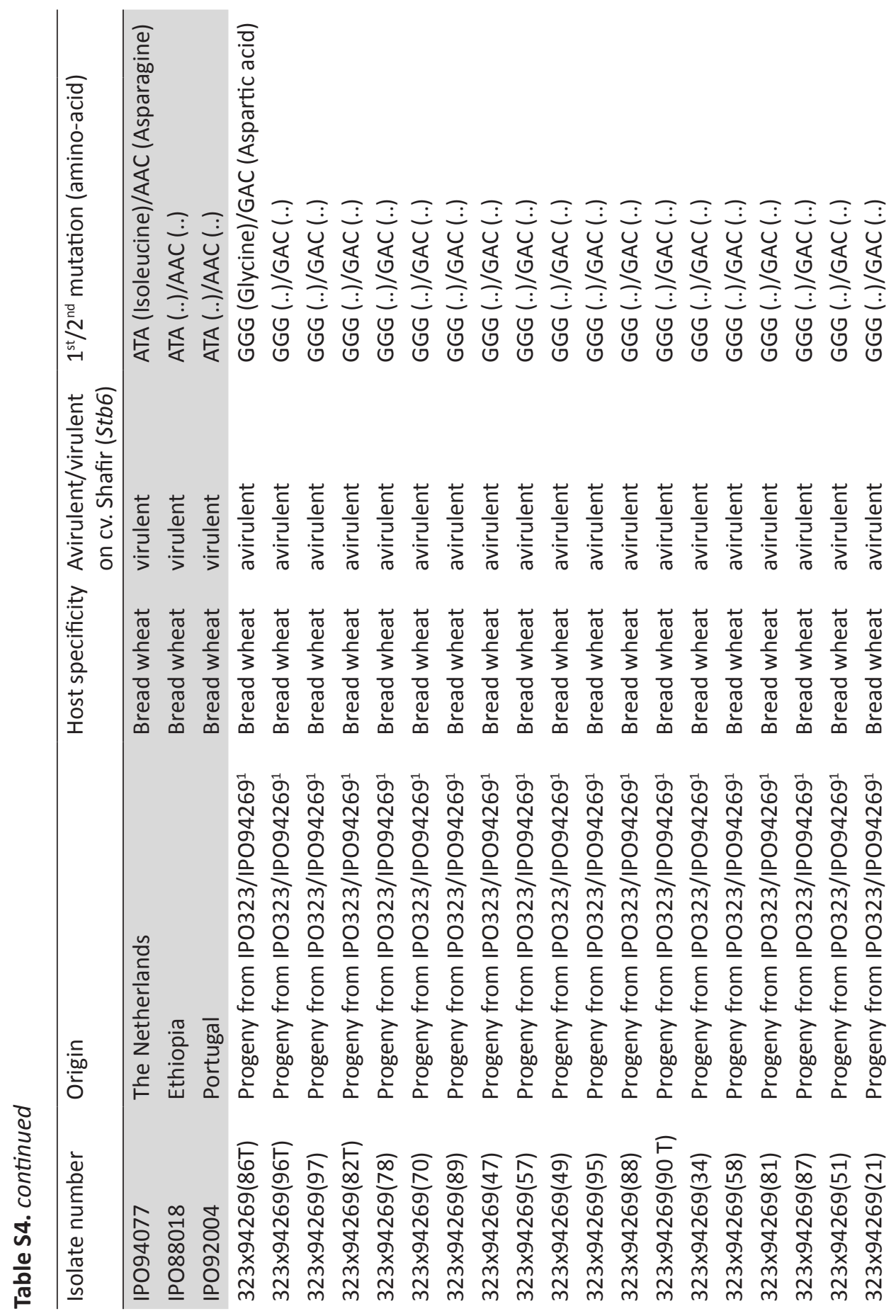




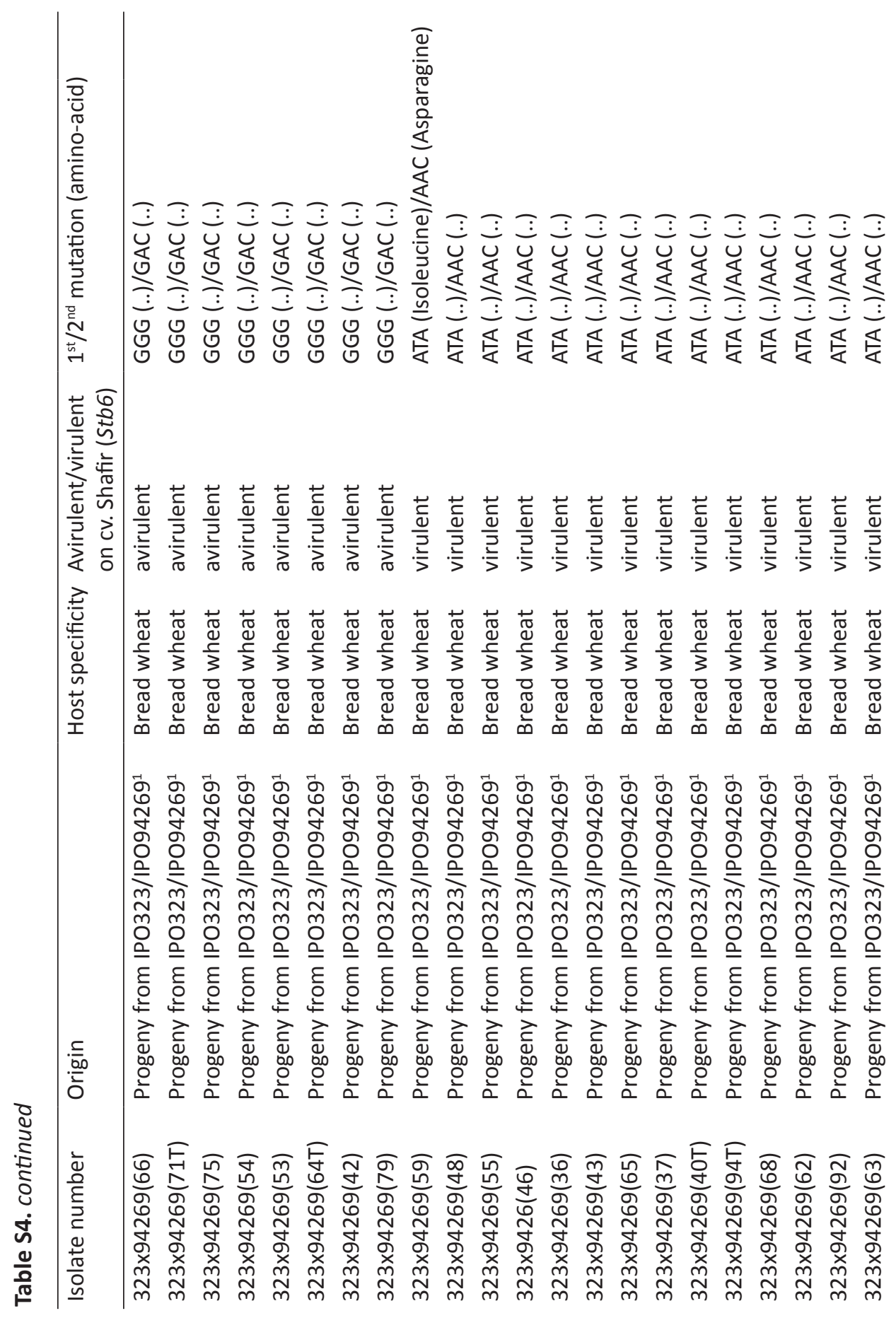




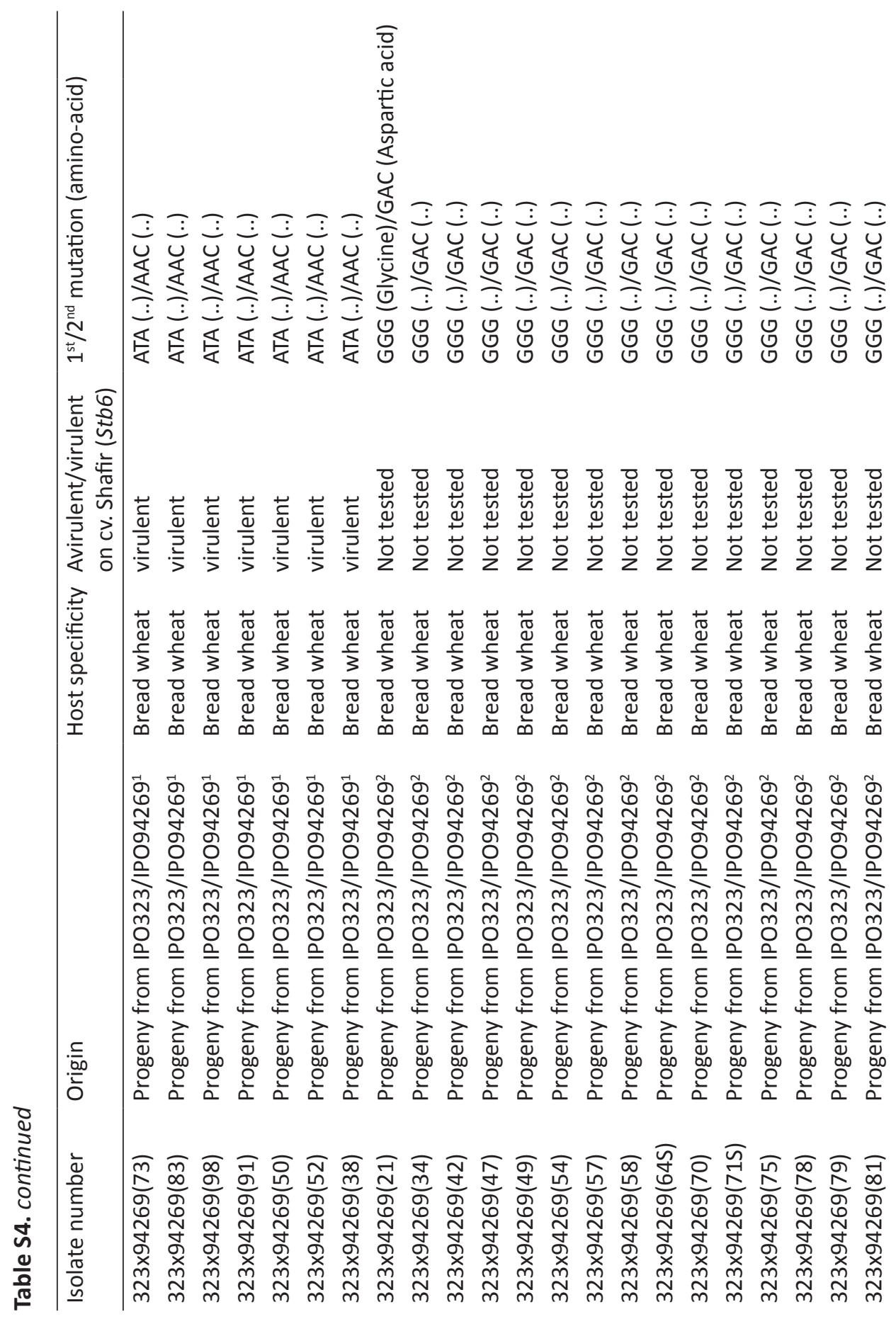




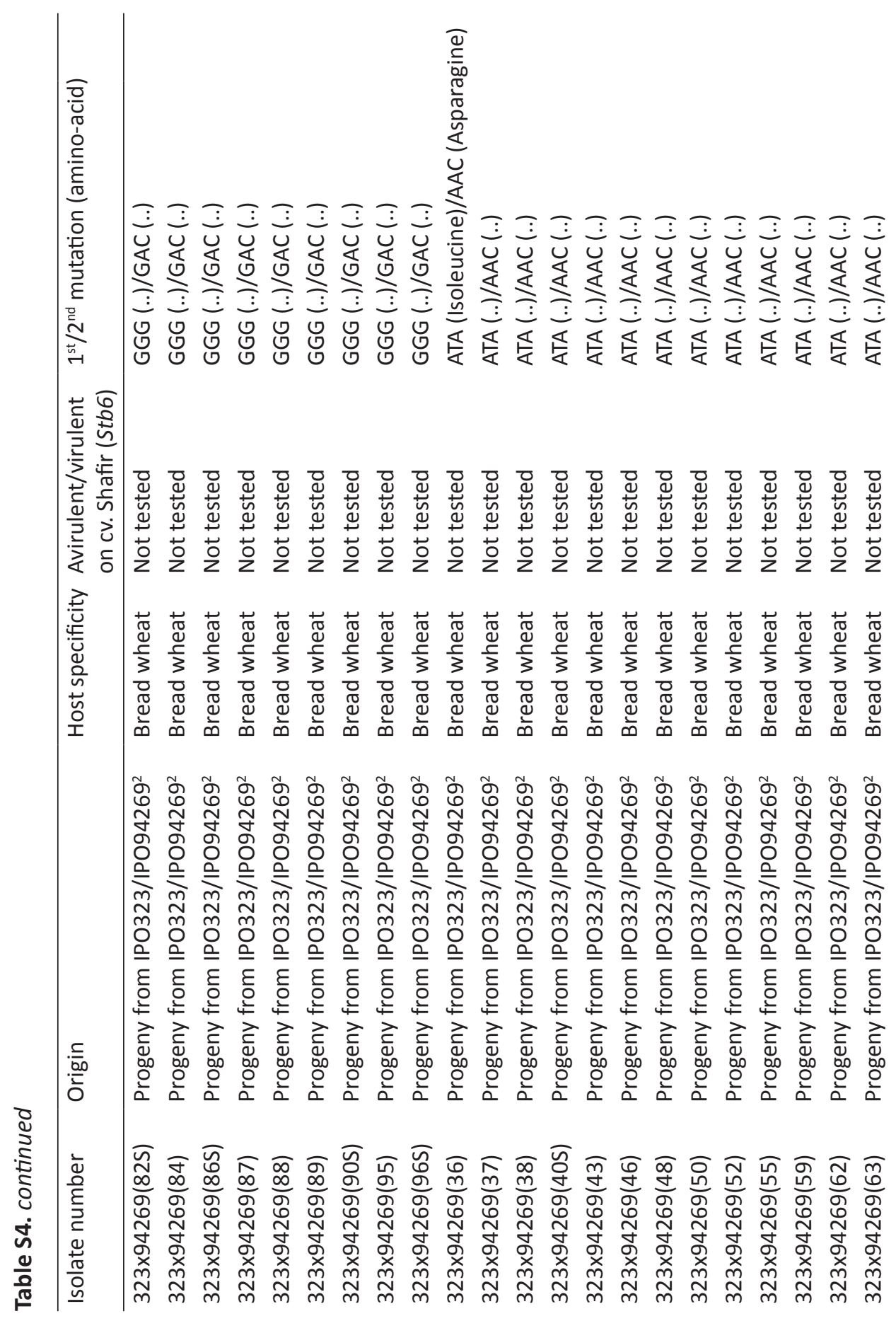




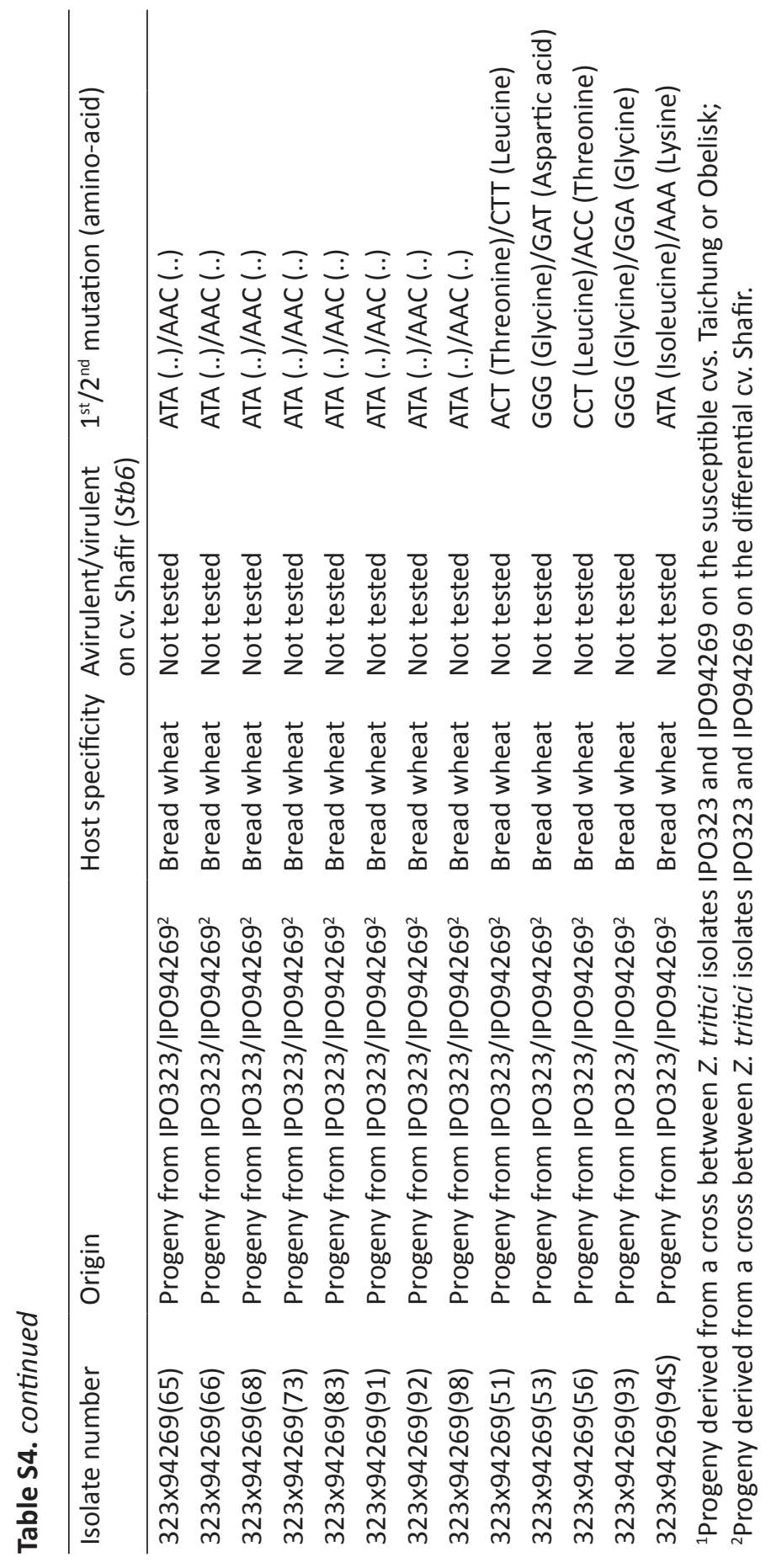


Table S5. Summary information about the Zymoseptoria tritici isolates used in the in planta crossing protocol under various levels of preventative strobilurin applications.

\begin{tabular}{llllll}
\hline Pycnidial isolate & Location & Year & $\begin{array}{l}\text { Strobilurin } \\
\text { application }\end{array}$ & $\begin{array}{l}\text { Strobilurin } \\
\text { phenotype }\end{array}$ & $\begin{array}{l}\text { Mating } \\
\text { type }\end{array}$ \\
\hline IPO03001 (BCS3R) & Germany & 2003 & unknown & Resistant & mat1-1 \\
IPO03002 (BCS8S) & Germany & 2003 & unknown & Resistant & mat1-2 \\
IP003003 (BCS16S) & Germany & 2003 & unknown & Sensitive & mat1-2 \\
IPO03005 (BCS17S) & Germany & 2003 & unknown & Sensitive & mat1-1 \\
IP004001 & Netherlands & 2004 & No & Sensitive & mat1-1 \\
IP004011 & Netherlands & 2004 & No & Resistant & mat1-2 \\
IPO323 (reference) & Netherlands & 1981 & No & Sensitive & mat1-1 \\
IP094269 (reference) & Netherlands & 1994 & No & Sensitive & mat1-2 \\
\hline
\end{tabular}

aisolate code from Bayer Cropscience; ${ }^{b}$ phenotype of isolate was mislabeled. Sequence information and additional Phenotyping confirmed its resistance to strobilurin.

Table S6. Probes and primers for Zymoseptoria tritici used in this study.

\begin{tabular}{ll}
\hline Name & Sequence (5' to 3') \\
\hline MAT1-1 F & CCGCTTTCTGGCTTCTTCGCACTG \\
MAT1-1 R & TGGACACCATGGTGAGAGAACCT \\
MAT1-2 F & GGCGCCTCCGAAGCAACT \\
MAT1-2 R & GATGCGGTTCTGGACTGGAG \\
StrobSNP2fwd & CTTATGGTCAAATGTCTTTATGATG \\
StrobSNP1rvs & GGTGACTCAACGTGATAGC \\
StrobSNPrcF7 & CAATAAGTTAGTTATAACTGTTGCGG \\
StrobSNPrCR1 & CTATGCATTATAACCCTAGCGT \\
Mmat1P3 & FAM- CGCAGTCTGCTTTGAATGAGAAGTTATC-Darquencher \\
Mmat1F3 & GGCATTTCGCAGTATGTG \\
Mmat1R3 & CTGCGCATTTCTCGTC \\
Mmat2P4 & YY- CCTCGCAAGCCATCGGAGA -Darquencher \\
Mmat2F7 & GCATCCGGGATACCAGTA \\
Mmat2R7 & CTTGGTCATGCGACGTT \\
ag-0003 F & ACTTGGGGAGGTGTTGTGAG \\
ag-0003 R & ACGAATTGTTCATTCCAGCG \\
gca-0004 F & TAACGGTAACGGCAACAACC \\
\hline
\end{tabular}


Table S6. continued

\begin{tabular}{ll}
\hline Name & Sequence (5' to 3') $^{\prime}$ \\
\hline gca-0004 R & GTGTACCCTTGAATCGCAGC \\
tcc-0008 F & AAAAGACATGACGCCCGAC \\
tcc-0008 R & ACGAGGAATAATCGCGGAAC \\
ag-0006 F & TAACCAACACCAGGGGAATG \\
ag-0006 R & CATCAGTTGTCAGCGAATGG \\
ag-0009 F & GACTCCATTTACCTGTGGCG \\
ag-0009 R & TGTGAAGGACACGCAAAGAG \\
tcc-0006 F & ATCTGGACACCATCCACCAG \\
tcc-0006 R & GTAGGTGGGAGGGTTCATGC \\
ac-0001 F & CACCACACCGTCGTTCAAG \\
ac-0001 R & CGTAAGTTGGTGGAGATGGG \\
ggc-0001F & GATACCAAGGTGGCCAAGG \\
ggc-0001R & CACGTTGGGAGTGTCGAAG \\
caa-0002 F & TCTGCAGAGATCCCGTTACC \\
caa-0002 R & ATCCATCACATGACGCACAC \\
ac-0007 F & TGCTCGCAAGACATAAAACG \\
ac-0007 R & CTCTTAGCATTGGTCGGTGG \\
ZtAvrSt6-F & TTCCACACTTCTTTCCACAACTCC \\
ZtAvrSt6-R & CATGCAATGGAGGTATGTATGGG \\
ZtAvrStb6-F1 & GTACACTTGTTTAGAGGTAATCCTTC \\
ZtAvrStb6-R1 & GTCGTCGTCGTCGCAATTGATAA \\
Q- ZtAvrSt6-F & TTCCAGGACGGGCAATATC \\
Q- ZtAvrSt6-R & AGCCACAACCAAGAATGACC \\
Mt-SSR-F & CTCAGTTCAAGTCTGAGTGC \\
Mt-SSR-R & GACGCACGCATTTCCACTCTA \\
\hline
\end{tabular}


Table S7. Natural and agricultural systems for Leonard's model and EEP model.

\begin{tabular}{|c|c|c|}
\hline & Leonard's model & EEP model \\
\hline \multirow{3}{*}{$\begin{array}{l}\text { Natural } \\
\text { system }\end{array}$} & $n_{n_{i+1}}=\frac{n_{i}(1-k)}{}$ & $\left(1-q_{i}^{2}\right)(1-k) n_{i}\left[\frac{\sigma+(1-k-\sigma) n_{i}}{2 \sigma+(1-k-2 \sigma) n_{i}}\right]+q_{i}^{2}(1-k) n_{i}$ \\
\hline & $n_{i+1}=\overline{q_{i}^{2}+n_{i}\left(\left(1-q_{i}^{2}\right)-k\right)}$ & $n_{i+1}=\frac{\left[2 \sigma+(1-k-2 \sigma) n_{i}\right]}{q_{i}^{2}+n_{i}\left(\left(1-q_{i}^{2}\right)-k\right)}$ \\
\hline & $p_{i}\left[1-d-n_{i} c(1-k)\right]$ & $p_{i}\left[1-d-n_{i} c(1-k)\right]$ \\
\hline
\end{tabular}

Agricultural system

$$
\begin{array}{ll}
n_{i+1}=\frac{n_{i}(1-k)}{q^{2}+n_{i}\left(\left(1-q^{2}\right)-k\right)} & \\
n_{i+1}=q_{i}=q & q_{i+1}=q_{i}=q
\end{array}
$$




\section{REFERENCES}

Adhikari, T.B., Anderson, J.M. \& Goodwin, S.B. Identification and molecular mapping of a gene in wheat conferring resistance to Mycosphaerella graminicola. Phytopathology 93, 1158-1164 (2003).

Adhikari, T.B. et al. Molecular mapping of the Stb4 gene for resistance to septoria tritici blotch in wheat. Phytopathology 94, 1198-1206 (2004a).

Adhikari, T.B. et al. Molecular mapping of Stb1, a potentially durable gene for resistance to septoria tritici blotch in wheat. Theoretical and Applied Genetics 109, 944-953 (2004b).

Arraiano, L.S., Worland, A.J., Ellerbrook, C. \& Brown, J.K.M. Chromosomal location of a gene for resistance to septoria tritici blotch (Mycosphaerella graminicola) in the hexaploid wheat 'Synthetic 6x'. Theoretical and Applied Genetics 103, 758-764 (2001).

Arraiano, L.S. et al. A gene in European wheat cultivars for resistance to an African isolate of Mycosphaerella graminicola. Plant Pathology 56, 73-78 (2007).

Brading, P.A., Verstappen, E.C.P., Kema, G.H.J. \& Brown, J.K.M. A gene-for-gene relationship between wheat and Mycosphaerella graminicola, the septoria tritici blotch pathogen. Phytopathology 92, 439-445 (2002).

Chartrain, L., Sourdille, P., Bernard, M. \& Brown, J.K.M. Identification and location of Stb9, a gene for resistance to septoria tritici blotch in wheat cultivars Courtot and Tonic. Plant Pathology 58, 547-555 (2009).

Chartrain, L. et al. Genetics of resistance to septoria tritici blotch in the Portuguese wheat breeding line TE 9111. Theoretical and Applied Genetics 110, 1138-1144 (2005c).

Cowling, S.G. University of Manitoba (2006).

Cuthbert, R. University of Manitoba (2011).

Goodwin, S.B. \& Thompson, I. Development of isogenic lines for resistance to septoria tritici blotch in wheat. Czech Journal of Genetics and Plant Breeding 47, S98-S101 (2011).

Jing, $\mathrm{H}$. et al. Phenotypic and genetic analysis of the Triticum monococcum: Mycosphaerella graminicola interaction. The New Phytologist 179, 1121-1132 (2008).

Liu, Y., Zhang, L., Thompson, I.A., Goodwin, S.B. \& Ohm, H.W. Molecular mapping relocates the Stb2 gene for resistance to septoria tritici blotch derived from cultivar Veranopolis on wheat chromosome 1BS. Euphytica 190, 145-156 (2013).

McCartney, C.A., Brûlé-Babel, A.L., Lamari, L. \& Somers, D.J. Chromosomal location of a race-specific resistance gene to Mycosphaerella graminicola in the spring wheat ST6. Theoretical and Applied Genetics 107, 1181-1186 (2003).

Raman, R. et al. Molecular mapping and physical location of major gene conferring seedling resistance to septoria tritici blotch in wheat. Molecular Breeding 24, 153164 (2009). 
Somasco, O.A., Qualset, C.O. \& Gilchrist, D.G. Single-gene resistance to septoria tritici blotch in the spring wheat cultivar 'Tadinia'. Plant Breeding 115, 261-267 (1996).

Tabib Ghaffary, S.M. et al. New broad-spectrum resistance to septoria tritici blotch derived from synthetic hexaploid wheat. Theoretical and Applied Genetics 124, 125-142 (2012).

Tabib Ghaffary, S.M. et al. Genetic analysis of resistance to septoria tritici blotch in the French winter wheat cultivars Balance and Apache. Theoretical and Applied Genetics 123, 741-754 (2011). 


\section{Chapter 7}

$$
\text { d) }
$$

$$
26
$$

ᄂ

$d \sigma$ 
General discussion:

Effector repertoires of plant pathogens

revealed by comparative genomics 


\begin{abstract}
Plant pathogens cause substantial crop losses, thereby challenging agricultural efforts to meet the current and future global food security needs of the growing world population. During the infection process, pathogens secrete effector proteins that enable host plant colonization. Over the last decade, phytopathologists have sequenced the genomes of many plant pathogens and conducted genome comparisons of multiple isolates of their species of interest, or of a strain of their species with strains of related species, to investigate effector gene dynamics and evolution. Such studies have revealed that effector genes often localize within dynamic genomic regions and shown how pathogens adapt their effector gene repertoires to their hosts. Moreover, comparative genomic studies allowed the determination of effector gene repertoires of plant pathogens and the subsequent use of such effectors to screen for recognition specificities in host plant germplasm. In this final chapter of my thesis, I will discuss some of the approaches to discover effectors, how pathogens adapt their effector catalogs to their hosts, and how the discovery of effectors can be exploited to develop durable resistance breeding strategies.
\end{abstract}




\section{INTRODUCTION}

Plant diseases, caused by pathogens and pests, impact food security and are a continuous and a major challenge for agriculture worldwide (Fisher et al. 2012). To establish their infection, pathogens secrete an arsenal of effector proteins, many of which aim to modulate host physiology, including components of the host immune system (Cook et al. 2015; Jones and Dangl 2006). However, in turn, effectors may become recognized by host immune receptors that activate strong defence responses aimed at re-instalment of immunity (Cook et al. 2015; Jones and Dangl 2006). Consequently, pathogens need to mutate or purge recognized effectors, or evolve novel ones to suppress immunity and successfully colonize the plant (Cook et al. 2015; Jones and Dangl 2006).

Over the last decade, genome comparisons of multiple isolates of a particular species of interest, or of their species of interest with related species, identified effector genes and investigated effector gene dynamics and evolution (Duplessis et al. 2011; Kim et al. 2016; Soanes et al. 2008; Sperschneider et al. 2014; Stukenbrock et al. 2011). Such effector genes are often localized within highly dynamic genomic regions that facilitate effector gene evolution to enable pathogens to escape or overcome host immunity (Dong et al. 2015; Raffaele and Kamoun 2012). Moreover, effector gene repertoires determine the host range of a particular plant pathogen (Ailloud et al. 2015; Baroncelli et al. 2016; Dong et al. 2014; Liao et al. 2016; van Dam et al. 2016). Here, I discuss some of the approaches to identify effector genes and reveal how pathogens adapt their effector gene repertoires to their host plants. Moreover, I discuss how the discovery of effectors can be exploited to develop novel durable disease management strategies.

\section{EFFECTORS OF PLANT PATHOGENS REVEALED BY COMPARATIVE GENOMICS}

Effector genes are often not randomly distributed over the genome (Haas et al. 2009; Raffaele et al. 2010; Rouxel et al. 2011). Rather, effector genes are typically localized within plastic genomic regions that are enriched for repeats and that exhibit structural variations such as presence/absence polymorphisms or small- and large-scale rearrangements (Dong et al. 2015; Raffaele and Kamoun 2012). These plastic genomics regions may occur on separate chromosomes that are generally smaller than the core chromosomes and are not required for normal growth and physiology, often referred to as conditionally dispensable chromosomes (CDCs), in particular pathogen species such as in the tomato wilt pathogen Fusarium oxysporum f. sp. lycopersici (Ma et al. 2010; Vlaardingerbroek et al. 2016), the Brassicaceae blackleg pathogen Leptosphaeria maculans (Balesdent et al. 2013; Rouxel et al. 2011), and in pathogenic Alternaria species (Akagi et al. 2009; Akamatsu et al. 1999). Alternatively, plastic genomic regions are embedded within core chromosomes, as shown for the maize smut pathogen Ustilago maydis (Kamper et al. 2006; Schirawski et al. 2010), but also for the broad host range pathogen Verticillium dahliae (de Jonge et al. 2013; de Jonge et al. 2012; Faino et 
al. 2016). By comparing the genomes of multiple isolates of a pathogen of interest, core effector genes that are shared by all strains (de Jonge et al. 2013; Hemetsberger et al. 2015; Marshall et al. 2011; Meile et al. 2018; Stergiopoulos et al. 2010) and lineagespecific (LS) effector genes that are only shared by a subset of strains can be identified (de Jonge et al. 2013; Schmidt et al. 2013). In this thesis, we performed comparative genomics of $21 \mathrm{~V}$. dahliae strains and determined the core and LS effector repertoires of these strains (Figure $1 \mathrm{~A}$ ) (Chapter 3). Intriguingly, we observed that $V$. dahliae strains that are able to infect the same host plant harbour highly divergent LS effector repertoires with not even a single shared LS effector (Chapter 3 ). This has led us to suggest that different $V$. dahliae strains are able to infect the same host plant by utilizing different effectors, suggesting redundancy among the activities of effector repertoires between lineages (Chapter 3). Furthermore, we assessed the expression of core $V$. dahliae effector genes, as core effectors can play essential roles on a multitude of hosts (Guyon et al. 2014; Hemetsberger et al. 2015; Santhanam et al. 2013; Yin et al. 2017), and observed differential expression of $V$. dahliae core effector genes between host plants (Chapter 3). Moreover, we compared features of $V$. dahliae core and LS effector genes and observed that LS effector genes localize significantly closer to transposable elements (TEs) when compared with core effector genes (Chapter 3), which may hint towards accelerated evolution of the LS effector repertoires facilitated by TEs (Seidl and Thomma 2017). Similarly, comparative genomics of 136 Zymoseptoria tritici isolates revealed that LS effector genes localize significantly closer to TEs when compared with core effector genes, suggesting rapid evolution of $Z$. tritici LS effector genes (Chapter 5 ). However, thus far, all identified $Z$. tritici effector genes are localized within core genomic regions (Figure 1B) (McDonald et al. 2015), while effector genes localized within core and LS regions have been shown to play a role in $V$. dahliae virulence on various host plants (de Jonge et al. 2013; de Jonge et al. 2012; Kombrink et al. 2016; Santhanam et al. 2013). Thus, comparative genomics is a powerful tool that aids the identification of core and LS effector repertoires and can provide insights into the evolution of virulence of the pathogen of interest. 
A

Verticillium dahliae

B

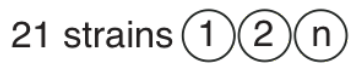

Core
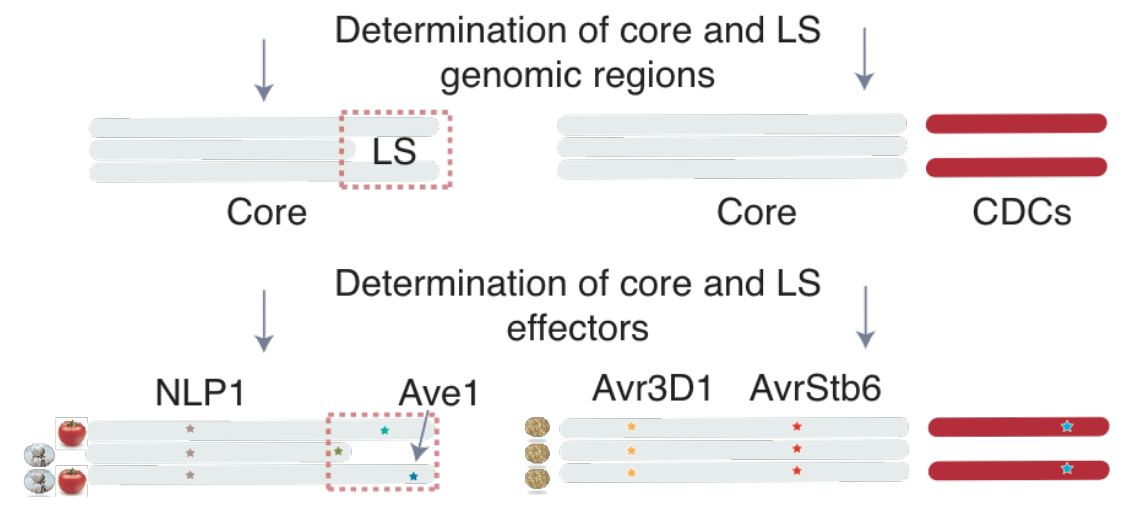

\section{Zymoseptoria tritici}

136 strains (1) (2)

\section{6 strains 120}

Figure 1. Effector gene repertoires revealed by comparative genomics. A) Effector genes (star) localized within the core genome (e.g. NLP1) or within lineage-specific (LS) genomic regions (e.g. Ave1) are indicated for Verticillium dahliae strains. Host plants, for example cotton and tomato, that the $V$. dahliae strain is capable to infect are indicated by the cotton flower and tomato symbol, respectively. B) Effector genes localized on core or on conditionally dispensable chromosomes (CDCs) are indicated for Zymoseptoria tritici strains. All thus far known effector genes, for example AvrStb6 and Avr3D1, are localized on core chromosomes.

Once an effector gets recognized by a matching plant immune receptor, turning the effector into an avirulence (Avr) factor, pathogens need to eliminate or modify that effector to overcome host immunity (Cook et al. 2015; Jones and Dangl 2006). To this end, deletion of the entire effector gene (Balesdent et al. 2013; de Jonge et al. 2012; Hartmann et al. 2017; Schurch et al. 2004; Stukenbrock and McDonald 2007), or sequence modifications, even as subtle as a single nucleotide polymorphism (SNP) (Bhadauria et al. 2015; Joosten et al. 1994), may occur in the protein coding sequence of the effector gene. The deletion of an Avr effector gene can typically be revealed by comparative genomics between virulent and avirulent strains to identify genomic regions that are absent in virulent strains while present in avirulent strains. For example, comparative genomics between $V$. dahliae strains that are virulent and avirulent on tomato plants that carry the Ve1 immune receptor revealed a $50 \mathrm{~kb}$ LS region that is present in avirulent strains and absent in virulent ones, harboring the effector gene Ave1 (de Jonge et al. 2012). This effector was subsequently experimentally shown to be the avirulence effector that is recognized by the tomato immune receptor Ve1 (de 
Jonge et al. 2012). The AVRFOM2 effector of the melon wilt fungus Fusarium oxysporum f. sp. melonis that is recognized by melon Fom-2 was identified using a similar approach (Schmidt et al. 2016). However, even when recognition escape is mediated by a SNP in an Avr effector gene, comparative genomics between virulent and avirulent strains may be used to identify the avirulence effector gene. For example, for the tomato leaf mold fungus Cladosporium fulvum, comparative transcriptomics was performed on avirulent as well as virulent strains during infection of tomato plants carrying the $\mathrm{Cf}-5$ immune receptor, identifying 44 in planta-induced effector genes (Mesarich et al. 2014). Subsequently, sequence comparisons between these effector genes revealed two that carry a SNP that is shared by virulent strains but absent in avirulent ones and that alters the protein-coding sequence (Mesarich et al. 2014). One of these effectors was termed Avr5 because follow-up experiments revealed that it is recognized by the tomato immune receptor Cf-5 (Mesarich et al. 2014). Similarly, we conducted comparative genomics between $Z$. tritici isolates that are virulent and avirulent on the wheat cultivar Shafir that carries the resistance protein Stb6 and identified a polymorphic genomic region of $250 \mathrm{~kb}$ (Kema et al. 2018; Chapter 6). This genomic region harbors the effector gene AvrStb6 that occurs in nearly all Z. tritici isolates (except for a single isolate) and displays non-synonymous SNPs in strains that are virulent on Shafir (Kema et al. 2018; Chapter 5 and 6). Thus, sequence modifications or deletions of Avr effector genes are common in plant pathogens (Dodds et al. 2006; Gout et al. 2007; lida et al. 2015; Poppe et al. 2015; Schurch et al. 2004), which highlights the importance of comparative genomics to reveal such mutated or purged effector genes in a pathogen of interest.

\section{PATHOGENS ADAPT THEIR EFFECTOR GENE REPERTOIRES TO THEIR HOST PLANTS}

The co-evolutionary arms race between plants and pathogens, in which pathogens continuously diversify their effector repertoires to avoid recognition by plant immune receptors while plants simultaneously evolve novel receptors to intercept pathogens, may lead to specialized pathogens that are capable to cause disease on a particular host (Kanzaki et al. 2012; Liao et al. 2016). However, some pathogens are still capable to cause disease on a multitude of hosts (Cook et al. 1980; Fradin and Thomma 2006; Kirk et al. 2009; Michielse and Rep 2009), yet it remains unknown how such pathogens adapt their effector gene repertoires to be competitive in arms races with diverse host plants.

Over the past few years, multiple studies have suggested that the host range of a particular plant pathogen is governed by its effector gene repertoire (Chiapello et al. 2015; Liao et al. 2016; Ma et al. 2010; van Dam et al. 2016). Thus, plant pathogens may harbour specialized effectors that exert their activities only on particular hosts. The role of specialized pathogen effectors was shown for the closely related oomycete pathogens Phytophtora infestans, which is pathogenic on members of the genus Solanum, and $P$. 
mirabilis, which is pathogenic on Mirabilis jalapa (Dong et al. 2014). These two pathogens harbour genes that encode apoplastic EPIC effectors that exert their activities only on their respective hosts (Dong et al. 2014). Similarly, the $V$. dahliae effector protein NLP2 (necrosis-and ethylene-inducing-like protein 2) promotes virulence of $V$. dahliae strain JR2 on tomato and Arabidopsis thaliana, but not on Nicotiana benthamiana (Santhanam et al. 2013). In this thesis, we furthermore showed that the Sun1 effector contributes to aggressiveness of $V$. dahliae strain $85 \mathrm{~S}$ only on sunflower, but not on $N$. benthamiana or A. thaliana (Chapter 4). Moreover, plant pathogens may harbour groups of specialized effectors that allow them to establish disease on particular hosts. For example, formae speciales of $F$. oxysporum, which indicates on which host plant an isolate is pathogenic, harbour groups of specialized effectors that contribute to pathogen virulence only on a particular host (Ma et al. 2010; van Dam et al. 2016). However, other plant pathogens may harbour a number of effectors that contribute to disease development on various hosts. For example, the effector protein NLP1 (necrosis-and ethylene-inducing-like protein 1) of $V$. dahliae has been shown to promote virulence on multiple host plants including tomato, $N$. benthamiana, and $A$. thaliana (Santhanam et al. 2013). Collectively, these examples illustrate that pathogens adapt their effector gene repertoires to specific or various host plants to establish disease.

\section{EFFECTORS CAN BE USED TO SCREEN FOR RESISTANCE IN HOST PLANTS}

Breeding for disease resistance can be achieved through utilizing effectors of plant pathogens (Lauge et al. 1998; Vleeshouwers and Oliver 2014). This can be achieved by transient expression of candidate effector genes in plant leaves by Agrobacteriummediated expression and by a virus vector such as Potato virus X (Chapman et al. 1992; Janssen and Gardner 1990). Subsequently, plants can be screened for the occurrence of cell death-associated defence reaction, known as the hypersensitive response, which indicates effector recognition by a matching plant immune receptor (Jones and Dangl 2006). To screen for resistance, it may not be advisable to use LS effectors, as these are strain specific and could be absent in a subset of pathogen strains that may immediately break resistance. Alternatively, core effectors can be used as these may be essential for particular plant pathogens and may therefore less easily be lost. For example, the core $C$. fulvum ECP2 effector protein was used to screen for hypersensitive response among a collection of tomato genotypes, which led to the identification of the matching immune receptor Cf-ECP2 (Lauge et al. 1998). As this immune receptor recognizes a potentially important virulence factor, it may be utilized to aid for durable resistance against $C$. fulvum (Lauge et al. 1998). Thus, identification and exploitation of plant resistance proteins that recognize core effectors might potentially lead to durable pathogen resistance.

Over the past few years, many resistance genes have been identified and deployed in crop plants to limit disease development (Fu et al. 2009; Gómez-Gómez and Boller 
2000; Hann and Rathjen 2007; Hurni et al. 2013; Saintenac et al. 2018; Zhang et al. 2017). However, resistance is often broken as pathogens evolve mechanisms to eliminate or modify particular effectors to overcome resistance. One mechanism to diversify effector repertoires is through sexual reproduction (Seidl and Thomma 2014), where the recombination of effector gene repertoires between two parents may lead to novel allele combinations allowing pathogens to evade the host immunity. In Z. tritici, sexual reproduction can even occur with an avirulent parent carrying the avirulence effector gene AvrStb6 on the resistant host that carries Stb6, retaining AvrStb6 in subsequent populations (Chapter 6). However, for various plant pathogens sex has never been observed, yet these pathogens are able to overcome host resistance. For example, the asexual pathogen $C$. fulvum overcomes resistance of tomato plant carrying the Cf-9 immune receptor through deletion of the avirulence effector gene Avr9 (van Kan et al. 1991). Similarly, deletion of the avirulence effector gene Ave1 allows strains of the asexual pathogen $V$. dahliae to overcome resistance of tomato plants carrying the Ve1 immune receptor (de Jonge et al. 2012). Thus, deletion of avirulence effector genes is one of the mechanisms that allows sexual as well as asexual plant pathogens to evade host immunity. Over the years, plant breeders have learned that the deployment of single resistance genes may lead to rapid degeneration of resistance due to the rapid emergence of resistance-breaking pathogen strains. Thus, novel resistance sources should be deployed wisely in order to use them in a sustainable manner. One of the strategies to deploy resistance in a sustainable manner relies on stacking multiple $R$ genes directed against the same pathogen strain in a single variety, thus increasing the difficulty for a pathogen to overcome resistance (Michelmore et al. 2013). Thus, efforts should be made to simultaneously identify multiple resistance sources against the same pathogen that can be combined into the crop of interest. 


\section{REFERENCES}

Ailloud, F., Lowe, T., Cellier, G., Roche, D., Allen, C., and Prior, P. 2015. Comparative genomic analysis of Ralstonia solanacearum reveals candidate genes for host specificity. BMC Genomics 16:270.

Akagi, Y., Akamatsu, H., Otani, H., and Kodama, M. 2009. Horizontal chromosome transfer, a mechanism for the evolution and differentiation of a plant-pathogenic fungus. Eukaryotic Cell 8:1732-1738.

Akamatsu, H., Taga, M., Kodama, M., Johnson, R., Otani, H., and Kohmoto, K. 1999. Molecular karyotypes for Alternaria plant pathogens known to produce hostspecific toxins. Current Genetics 35:647-656.

Balesdent, M.-H., Fudal, I., Ollivier, B., Bally, P., Grandaubert, J., Eber, F., Chèvre, A.-M., Leflon, M., and Rouxel, T. 2013. The dispensable chromosome of Leptosphaeria maculans shelters an effector gene conferring avirulence towards Brassica rapa. New Phytologist 198:887-898.

Baroncelli, R., Amby, D. B., Zapparata, A., Sarrocco, S., Vannacci, G., Le Floch, G., Harrison, R. J., Holub, E., Sukno, S. A., Sreenivasaprasad, S., and Thon, M. R. 2016. Gene family expansions and contractions are associated with host range in plant pathogens of the genus Colletotrichum. BMC Genomics 17:555.

Bhadauria, V., Maclachlan, R., Pozniak, C., and Banniza, S. 2015. Candidate effectors contribute to race differentiation and virulence of the lentil anthracnose pathogen Colletotrichum lentis. BMC Genomics 16:628.

Chapman, S., Kavanagh, T., and Baulcombe, D. 1992. Potato virus X as a vector for gene expression in plants. Plant Journal 2:549-557.

Chiapello, H., Mallet, L., Guerin, C., Aguileta, G., Amselem, J., Kroj, T., Ortega-Abboud, E., Lebrun, M. H., Henrissat, B., Gendrault, A., Rodolphe, F., Tharreau, D., and Fournier, E. 2015. Deciphering genome content and evolutionary relationships of isolates from the fungus Magnaporthe oryzae attacking different host plants. Genome Biology and Evolution 7:2896-2912.

Cook, D. E., Mesarich, C. H., and Thomma, B. P. H. J. 2015. Understanding plant immunity as a surveillance system to detect invasion. Annual Review of Phytopathology 53:541-563.

Cook, R., Sitton, J., and Waldher, J. 1980. Evidence for Pythium as a pathogen of directdrilled wheat in the Pacific Northwest. Plant Disease 64:61-61.

de Jonge, R., Bolton, M. D., Kombrink, A., van den Berg, G. C. M., Yadeta, K. A., and Thomma, B. P. H. J. 2013. Extensive chromosomal reshuffling drives evolution of virulence in an asexual pathogen. Genome Research 23:1271-1282.

de Jonge, R., Peter van Esse, H., Maruthachalam, K., Bolton, M. D., Santhanam, P., Saber, M. K., Zhang, Z., Usami, T., Lievens, B., Subbarao, K. V., and Thomma, B. P. H. J. 2012. Tomato immune receptor Ve1 recognizes effector of multiple fungal pathogens uncovered by genome and RNA sequencing. Proceedings of the National Academy of Sciences of the United States of America 109:5110-5115. 
Dodds, P. N., Lawrence, G. J., Catanzariti, A.-M., Teh, T., Wang, C.-I. A., Ayliffe, M. A., Kobe, B., and Ellis, J. G. 2006. Direct protein interaction underlies gene-for-gene specificity and coevolution of the flax resistance genes and flax rust avirulence genes. Proceedings of the National Academy of Sciences of the United States of America 103:8888-8893.

Dong, S., Raffaele, S., and Kamoun, S. 2015. The two-speed genomes of filamentous pathogens: waltz with plants. Current Opinion in Genetics \& Development 35:5765.

Dong, S., Stam, R., Cano, L. M., Song, J., Sklenar, J., Yoshida, K., Bozkurt, T. O., Oliva, R., Liu, Z., Tian, M., Win, J., Banfield, M. J., Jones, A. M. E., van der Hoorn, R. A. L., and Kamoun, S. 2014. Effector specialization in a lineage of the Irish potato famine pathogen. Science 343:552-555.

Duplessis, S., Cuomo, C. A., Lin, Y.-C., Aerts, A., Tisserant, E., Veneault-Fourrey, C., Joly, D. L., Hacquard, S., Amselem, J., Cantarel, B. L., Chiu, R., Coutinho, P. M., Feau, N., Field, M., Frey, P., Gelhaye, E., Goldberg, J., Grabherr, M. G., Kodira, C. D., Kohler, A., Kües, U., Lindquist, E. A., Lucas, S. M., Mago, R., Mauceli, E., Morin, E., Murat, C., Pangilinan, J. L., Park, R., Pearson, M., Quesneville, H., Rouhier, N., Sakthikumar, S., Salamov, A. A., Schmutz, J., Selles, B., Shapiro, H., Tanguay, P., Tuskan, G. A., Henrissat, B., Van de Peer, Y., Rouzé, P., Ellis, J. G., Dodds, P. N., Schein, J. E., Zhong, S., Hamelin, R. C., Grigoriev, I. V., Szabo, L. J., and Martin, F. 2011. Obligate biotrophy features unraveled by the genomic analysis of rust fungi. Proceedings of the National Academy of Sciences of the United States of America 108:9166-9171.

Faino, L., Seidl, M. F., Shi-Kunne, X., Pauper, M., van den Berg, G. C. M., Wittenberg, A. H. J., and Thomma, B. P. H. J. 2016. Transposons passively and actively contribute to evolution of the two-speed genome of a fungal pathogen. Genome Research 26:1091-1100.

Fisher, M. C., Henk, D. A., Briggs, C. J., Brownstein, J. S., Madoff, L. C., McCraw, S. L., and Gurr, S. J. 2012. Emerging fungal threats to animal, plant and ecosystem health. Nature 484:186-194.

Fradin, E. F., and Thomma, B. P. 2006. Physiology and molecular aspects of Verticillium wilt diseases caused by $V$. dahliae and V. albo-atrum. Molecular Plant Pathology 7:71-86.

Fu, D., Uauy, C., Distelfeld, A., Blechl, A., Epstein, L., Chen, X., Sela, H., Fahima, T., and Dubcovsky, J. 2009. A kinase-START gene confers temperature-dependent resistance to wheat stripe rust. Science 323:1357-1360.

Gómez-Gómez, L., and Boller, T. 2000. FLS2: an LRR receptor-like kinase involved in the perception of the bacterial elicitor flagellin in Arabidopsis. Molecular Cell 5:10031011.

Gout, L., Kuhn, M. L., Vincenot, L., Bernard-Samain, S., Cattolico, L., Barbetti, M., Moreno-Rico, O., Balesdent, M.-H., and Rouxel, T. 2007. Genome structure impacts molecular evolution at the AvrLm1 avirulence locus of the plant pathogen Leptosphaeria maculans. Environmental Microbiology 9:2978-2992. 
Guyon, K., Balagué, C., Roby, D., and Raffaele, S. 2014. Secretome analysis reveals effector candidates associated with broad host range necrotrophy in the fungal plant pathogen Sclerotinia sclerotiorum. BMC Genomics 15:336.

Haas, B. J., Kamoun, S., Zody, M. C., Jiang, R. H., Handsaker, R. E., Cano, L. M., Grabherr, M., Kodira, C. D., Raffaele, S., Torto-Alalibo, T., Bozkurt, T. O., Ah-Fong, A. M., Alvarado, L., Anderson, V. L., Armstrong, M. R., Avrova, A., Baxter, L., Beynon, J., Boevink, P. C., Bollmann, S. R., Bos, J. I., Bulone, V., Cai, G., Cakir, C., Carrington, J. C., Chawner, M., Conti, L., Costanzo, S., Ewan, R., Fahlgren, N., Fischbach, M. A., Fugelstad, J., Gilroy, E. M., Gnerre, S., Green, P. J., Grenville-Briggs, L. J., Griffith, J., Grunwald, N. J., Horn, K., Horner, N. R., Hu, C. H., Huitema, E., Jeong, D. H., Jones, A. M., Jones, J. D., Jones, R. W., Karlsson, E. K., Kunjeti, S. G., Lamour, K., Liu, Z., Ma, L., Maclean, D., Chibucos, M. C., McDonald, H., McWalters, J., Meijer, H. J., Morgan, W., Morris, P. F., Munro, C. A., O’Neill, K., Ospina-Giraldo, M., Pinzon, A., Pritchard, L., Ramsahoye, B., Ren, Q., Restrepo, S., Roy, S., Sadanandom, A., Savidor, A., Schornack, S., Schwartz, D. C., Schumann, U. D., Schwessinger, B., Seyer, L., Sharpe, T., Silvar, C., Song, J., Studholme, D. J., Sykes, S., Thines, M., van de Vondervoort, P. J., Phuntumart, V., Wawra, S., Weide, R., Win, J., Young, C., Zhou, S., Fry, W., Meyers, B. C., van West, P., Ristaino, J., Govers, F., Birch, P. R., Whisson, S. C., Judelson, H. S., and Nusbaum, C. 2009. Genome sequence and analysis of the Irish potato famine pathogen Phytophthora infestans. Nature 461:393-398.

Hann, D. R., and Rathjen, J. P. 2007. Early events in the pathogenicity of Pseudomonas syringae on Nicotiana benthamiana. The Plant Journal 49:607-618.

Hartmann, F. E., Sánchez-Vallet, A., McDonald, B. A., and Croll, D. 2017. A fungal wheat pathogen evolved host specialization by extensive chromosomal rearrangements. ISME Journal 27:2991-1204.

Hemetsberger, C., Mueller, A. N., Matei, A., Herrberger, C., Hensel, G., Kumlehn, J., Mishra, B., Sharma, R., Thines, M., Hückelhoven, R., and Doehlemann, G. 2015. The fungal core effector Pep1 is conserved across smuts of dicots and monocots. New Phytologist 206:1116-1126.

Hurni, S., Brunner, S., Buchmann, G., Herren, G., Jordan, T., Krukowski, P., Wicker, T., Yahiaoui, N., Mago, R., and Keller, B. 2013. Rye Pm8 and wheat Pm3 are orthologous genes and show evolutionary conservation of resistance function against powdery mildew. Plant Journal 76:957-969.

lida, Y., van 't Hof, P., Beenen, H., Mesarich, C., Kubota, M., Stergiopoulos, I., Mehrabi, R., Notsu, A., Fujiwara, K., Bahkali, A., Abd-Elsalam, K., Collémare, J., and De Wit, P. J. G. M. 2015. Novel mutations detected in avirulence genes overcoming tomato Cf resistance genes in Isolates of a Japanese population of Cladosporium fulvum. PLoS One 10:e0123271.

Janssen, B.-J., and Gardner, R. C. 1990. Localized transient expression of GUS in leaf discs following cocultivation with Agrobacterium. Plant Molecular Biology 14:61-72.

Jones, J. D. G., and Dangl, J. L. 2006. The plant immune system. Nature 444:323-329. 
Joosten, M. H. A. J., Cozijnsen, T. J., and De Wit, P. J. G. M. 1994. Host resistance to a fungal tomato pathogen lost by a single base-pair change in an avirulence gene. Nature 367:384-386.

Kamper, J., Kahmann, R., Bolker, M., Ma, L.-J., Brefort, T., Saville, B. J., Banuett, F., Kronstad, J. W., Gold, S. E., Muller, O., Perlin, M. H., Wosten, H. A. B., de Vries, R., Ruiz-Herrera, J., Reynaga-Pena, C. G., Snetselaar, K., McCann, M., Perez-Martin, J., Feldbrugge, M., Basse, C. W., Steinberg, G., Ibeas, J. I., Holloman, W., Guzman, P., Farman, M., Stajich, J. E., Sentandreu, R., Gonzalez-Prieto, J. M., Kennell, J. C., Molina, L., Schirawski, J., Mendoza-Mendoza, A., Greilinger, D., Munch, K., Rossel, N., Scherer, M., Vranes, M., Ladendorf, O., Vincon, V., Fuchs, U., Sandrock, B., Meng, S., Ho, E. C. H., Cahill, M. J., Boyce, K. J., Klose, J., Klosterman, S. J., Deelstra, H. J., Ortiz-Castellanos, L., Li, W., Sanchez-Alonso, P., Schreier, P. H., Hauser-Hahn, I., Vaupel, M., Koopmann, E., Friedrich, G., Voss, H., Schluter, T., Margolis, J., Platt, D., Swimmer, C., Gnirke, A., Chen, F., Vysotskaia, V., Mannhaupt, G., Guldener, U., Munsterkotter, M., Haase, D., Oesterheld, M., Mewes, H.-W., Mauceli, E. W., DeCaprio, D., Wade, C. M., Butler, J., Young, S., Jaffe, D. B., Calvo, S., Nusbaum, C., Galagan, J., and Birren, B. W. 2006. Insights from the genome of the biotrophic fungal plant pathogen Ustilago maydis. Nature 444:97-101.

Kanzaki, H., Yoshida, K., Saitoh, H., Fujisaki, K., Hirabuchi, A., Alaux, L., Fournier, E., Tharreau, D., and Terauchi, R. 2012. Arms race co-evolution of Magnaporthe oryzae AVR-Pik and rice Pik genes driven by their physical interactions. Plant Journal 72:894-907.

Kema, G. H. J., Mirzadi Gohari, A., Aouini, L., Gibriel, H. A. Y., Ware, S. B., van den Bosch, F., Manning-Smith, R., Alonso-Chavez, V., Helps, J., Ben M'Barek, S., Mehrabi, R., Diaz-Trujillo, C., Zamani, E., Schouten, H. J., van der Lee, T. A. J., Waalwijk, C., de Waard, M. A., De Wit, P. J. G. M., Verstappen, E. C. P., Thomma, B. P. H. J., Meijer, H. J. G., and Seidl, M. F. 2018. Stress and sexual reproduction affect the dynamics of the wheat pathogen effector AvrStb6 and strobilurin resistance. Nature Genetics 23:678.

Kim, K.-T., Jeon, J., Choi, J., Cheong, K., Song, H., Choi, G., Kang, S., and Lee, Y.-H. 2016. Kingdom-wide analysis of fungal small secreted proteins (SSPs) reveals their potential role in host association. Frontiers in Plant Sciences 7:186.

Kirk, W. W., Abu-El Samen, F., Tumbalam, P., Wharton, P., Douches, D., Thill, C. A., and Thompson, A. 2009. Impact of different US genotypes of Phytophthora infestans on potato seed tuber rot and plant emergence in a range of cultivars and advanced breeding lines. Potato Research 52:121-140.

Kombrink, A., Rovenich, H., Shi-Kunne, X., Rojas-Padilla, E., van den Berg, G. C. M., Domazakis, E., de Jonge, R., Valkenburg, D.-J., Sánchez-Vallet, A., Seidl, M. F., and Thomma, B. P. H. J. 2016. Verticillium dahliae LysM effectors differentially contribute to virulence on plant hosts. Molecular Plant Pathology 61:103. 
Lauge, R., Joosten, M. H. A. J., Haanstra, J. P. W., Goodwin, P. H., Lindhout, P., and De Wit, P. J. G. M. 1998. Successful search for a resistance gene in tomato targeted against a virulence factor of a fungal pathogen. Proceedings of the National Academy of Sciences of the United States of America 95:9014-9018.

Liao, J., Huang, H., Meusnier, I., Adreit, H., Ducasse, A., Bonnot, F., Pan, L., He, X., Kroj, T., Fournier, E., Tharreau, D., Gladieux, P., and Morel, J.-B. 2016. Pathogen effectors and plant immunity determine specialization of the blast fungus to rice subspecies. elife 5:e19377.

Ma, L.-J., van der Does, H. C., Borkovich, K. A., Coleman, J. J., Daboussi, M.-J., Di Pietro, A., Dufresne, M., Freitag, M., Grabherr, M., Henrissat, B., Houterman, P. M., Kang, S., Shim, W.-B., Woloshuk, C., Xie, X., Xu, J.-R., Antoniw, J., Baker, S. E., Bluhm, B. H., Breakspear, A., Brown, D. W., Butchko, R. A. E., Chapman, S., Coulson, R., Coutinho, P. M., Danchin, E. G. J., Diener, A., Gale, L. R., Gardiner, D. M., Goff, S., HammondKosack, K. E., Hilburn, K., Hua-Van, A., Jonkers, W., Kazan, K., Kodira, C. D., Koehrsen, M., Kumar, L., Lee, Y.-H., Li, L., Manners, J. M., Miranda-Saavedra, D., Mukherjee, M., Park, G., Park, J., Park, S.-Y., Proctor, R. H., Regev, A., Ruiz-Roldan, M. C., Sain, D., Sakthikumar, S., Sykes, S., Schwartz, D. C., Turgeon, B. G., Wapinski, I., Yoder, O., Young, S., Zeng, Q., Zhou, S., Galagan, J., Cuomo, C. A., Kistler, H. C., and Rep, M. 2010. Comparative genomics reveals mobile pathogenicity chromosomes in Fusarium. Nature 464:367-373.

Marshall, R., Kombrink, A., Motteram, J., Loza-Reyes, E., Lucas, J., Hammond-Kosack, K. E., Thomma, B. P. H. J., and Rudd, J. J. 2011. Analysis of Two in Planta Expressed LysM Effector homologs from the fungus Mycosphaerella graminicola reveals novel functional properties and varying contributions to virulence on wheat. Plant Physiology 156:756-769.

McDonald, M. C., McDonald, B. A., and Solomon, P. S. 2015. Recent advances in the Zymoseptoria tritici-wheat interaction: insights from pathogenomics. Frontiers in Plant Sciences 6:102.

Meile, L., Croll, D., Brunner, P. C., Plissonneau, C., Hartmann, F. E., McDonald, B. A., and Sánchez-Vallet, A. 2018. A fungal avirulence factor encoded in a highly plastic genomic region triggers partial resistance to septoria tritici blotch. New Phytologist 65:512.

Mesarich, C. H., Griffiths, S. A., van der Burgt, A., Ökmen, B., Beenen, H. G., Etalo, D. W., Joosten, M. H. A. J., and de Wit, P. J. G. M. 2014. Transcriptome sequencing uncovers the Avr5 avirulence gene of the tomato leaf mold pathogen Cladosporium fulvum. Molecular Plant-Microbe Interactions 27:846-857.

Michelmore, R. W., Christopoulou, M., and Caldwell, K. S. 2013. Impacts of resistance gene genetics, function, and evolution on a durable future. Annual Review of Phytopathology 51:291-319.

Michielse, C. B., and Rep, M. 2009. Pathogen profile update: Fusarium oxysporum. Molecular Plant Pathology 10:311-324. 
Poppe, S., Dorsheimer, L., Happel, P., and Stukenbrock, E. H. 2015. Rapidly evolving genes are key players in host specialization and virulence of the fungal wheat pathogen Zymoseptoria tritici (Mycosphaerella graminicola). PLoS Pathogens 11:e1005055.

Raffaele, S., and Kamoun, S. 2012. Genome evolution in filamentous plant pathogens: why bigger can be better. Nature Reviews Microbiology 10:417-430.

Raffaele, S., Win, J., Cano, L. M., and Kamoun, S. 2010. Analyses of genome architecture and gene expression reveal novel candidate virulence factors in the secretome of Phytophthora infestans. BMC Genomics 11:637.

Rouxel, T., Grandaubert, J., Hane, J. K., Hoede, C., van de Wouw, A. P., Couloux, A., Dominguez, V., Anthouard, V., Bally, P., Bourras, S., Cozijnsen, A. J., Ciuffetti, L. M., Degrave, A., Dilmaghani, A., Duret, L., Fudal, I., Goodwin, S. B., Gout, L., Glaser, N., Linglin, J., Kema, G. H. J., Lapalu, N., Lawrence, C. B., May, K., Meyer, M., Ollivier, B., Poulain, J., Schoch, C. L., Simon, A., Spatafora, J. W., Stachowiak, A., Turgeon, B. G., Tyler, B. M., Vincent, D., Weissenbach, J., Amselem, J., Quesneville, H., Oliver, R. P., Wincker, P., Balesdent, M.-H., and Howlett, B. J. 2011. Effector diversification within compartments of the Leptosphaeria maculans genome affected by repeat-Induced point mutations. Nature Communications 2:202.

Saintenac, C., Lee, W.-S., Cambon, F., Rudd, J. J., King, R. C., Marande, W., Powers, S. J., Bergès, H., Phillips, A. L., Uauy, C., Hammond-Kosack, K. E., Langin, T., and Kanyuka, K. 2018. Wheat receptor-kinase-like protein Stb6 controls gene-for-gene resistance to fungal pathogen Zymoseptoria tritici. Nature Genetics 51:291.

Santhanam, P., van Esse, H. P., Albert, I., Faino, L., Nürnberger, T., and Thomma, B. P. H. J. 2013. Evidence for functional diversification within a fungal NEP1-like protein family. Molecular Plant-Microbe Interactions 26:278-286.

Schirawski, J., Mannhaupt, G., Munch, K., Brefort, T., Schipper, K., Doehlemann, G., Di Stasio, M., Rossel, N., Mendoza-Mendoza, A., Pester, D., Muller, O., Winterberg, B., Meyer, E., Ghareeb, H., Wollenberg, T., Munsterkotter, M., Wong, P., Walter, M., Stukenbrock, E., Guldener, U., and Kahmann, R. 2010. Pathogenicity determinants in smut fungi revealed by genome comparison. Science 330:1546-1548.

Schmidt, S. M., Houterman, P. M., Schreiver, I., Ma, L., Amyotte, S., Chellappan, B., Boeren, S., Takken, F. L. W., and Rep, M. 2013. MITEs in the promoters of effector genes allow prediction of novel virulence genes in Fusarium oxysporum. BMC Genomics 14:1-21.

Schmidt, S. M., Lukasiewicz, J., Farrer, R., van Dam, P., Bertoldo, C., and Rep, M. C. 2016. Comparative genomics of Fusarium oxysporum f. sp. melonis reveals the secreted protein recognized by the Fom-2 resistance gene in melon. New Phytologist 209:307-318.

Schurch, S., Linde, C. C., Knogge, W., Jackson, L. F., and McDonald, B. A. 2004. Molecular population genetic analysis differentiates two virulence mechanisms of the fungal avirulence gene NIP1. Molecular Plant-Microbe Interactions 17:1114-1125.

Seidl, M. F., and Thomma, B. P. H. J. 2014. Sex or no sex: Evolutionary adaptation occurs regardless. BioEssays 36:335-345. 
Seidl, M. F., and Thomma, B. P. H. J. 2017. Transposable elements direct the coevolution between plants and microbes. Trends in Genetics:1-10.

Soanes, D. M., Alam, I., Cornell, M., Wong, H. M., Hedeler, C., Paton, N. W., Rattray, M., Hubbard, S. J., Oliver, S. G., and Talbot, N. J. 2008. Comparative genome analysis of filamentous fungi reveals gene family expansions associated with fungal pathogenesis. PLoS One 3:e2300.

Sperschneider, J., Ying, H., Dodds, P., Gardiner, D., Upadhyaya, N. M., Singh, K., Manners, J. M., and Taylor, J. 2014. Diversifying selection in the wheat stem rust fungus acts predominantly on pathogen-associated gene families and reveals candidate effectors. Frontiers in Plant Sciences 5.

Stergiopoulos, I., van den Burg, H. A., Okmen, B., Beenen, H. G., van Liere, S., Kema, G. H. J., and De Wit, P. J. G. M. 2010. Tomato Cf resistance proteins mediate recognition of cognate homologous effectors from fungi pathogenic on dicots and monocots. Proceedings of the National Academy of Sciences of the United States of America 107:7610-7615.

Stukenbrock, E. H., Bataillon, T., Dutheil, J. Y., Hansen, T. T., Li, R., Zala, M., McDonald, B. A., Wang, J., and Schierup, M. H. 2011. The making of a new pathogen: insights from comparative population genomics of the domesticated wheat pathogen Mycosphaerella graminicola and its wild sister species. Genome Research 21:21572166.

Stukenbrock, E. H., and McDonald, B. A. 2007. Geographical variation and positive diversifying selection in the host-specific toxin SnToxA. Molecular Plant Pathology 8:321-332.

van Dam, P., Fokkens, L., Schmidt, S. M., Linmans, J. H. J., Kistler, H. C., Ma, L.-J., and Rep, M. 2016. Effector profiles distinguish formae speciales of Fusarium oxysporum. Environmental Microbiology 18:4087-4102.

van Kan, J. A., van den Ackerveken, G. F., and de Wit, P.J. 1991. Cloning and characterization of cDNA of avirulence gene avr9 of the fungal pathogen Cladosporium fulvum, causal agent of tomato leaf mold. Molecular Plant-Microbe Interactions 4:52-59.

Vlaardingerbroek, I., Beerens, B., Schmidt, S. M., Cornelissen, B. J. C., and Rep, M. 2016. Dispensable chromosomes in Fusarium oxysporum f. sp. lycopersici. Molecular Plant Pathology 17:1455-1466.

Vleeshouwers, V. G., and Oliver, R. P. 2014. Effectors as tools in disease resistance breeding against biotrophic, hemibiotrophic, and necrotrophic plant pathogens. Molecular Plant-Microbe Interactions 27:196-206.

Yin, J., Gu, B., Huang, G., Tian, Y., Quan, J., Lindqvist-Kreuze, H., and Shan, W. 2017. Conserved RXLR effector genes of Phytophthora infestans expressed at the early stage of potato infection are suppressive to host defense. Frontiers in Plant Sciences 8:2155.

Zhang, W., Chen, S., Abate, Z., Nirmala, J., Rouse, M. N., and Dubcovsky, J. 2017. Identification and characterization of Sr13, a tetraploid wheat gene that confers resistance to the Ug99 stem rust race group. Proceedings of the National Academy of Sciences of the United States of America 114:E9483-E9492. 

Summary

Acknowledgements

About the author

List of publications

Education statement 



\section{SUMMARY}

During host colonization, plant pathogens secrete molecules that enable host colonization, also known as effector proteins. Over the last decade, considerable attention in research on plant-microbe interactions has focused on understanding how pathogens adapt their effector gene repertoires to their host plants. Chapter $\mathbf{1}$ is a literature study in which I describe the adaptation of the fungal pathogen Zymoseptoria tritici that is specialized on wheat plants, and compare that to the fungal pathogen Verticillium dahliae that is able to colonize a broad range of host plants. Additionally, I describe the main research questions and objectives of my $\mathrm{PhD}$ research, and provide an outline of the thesis.

Chapter 2 reviews how effector genes of plant pathogens can be discovered using genomics-based approaches. More specifically, I describe recent advances in genome sequencing technologies, genome assembly strategies, and gene annotations can reveal complete effector gene repertoires of plant pathogens. Moreover, I describe how the knowledge on effectors can be exploited to develop sustainable resistance breeding strategies.

Research on various pathogens has revealed that effector genes often localize within dynamic regions of their genomes, which is thought to facilitate accelerated evolution of effector (gene) catalogues. In Chapter 3, we determined effector genes localized within the core and lineage-specific (LS) genomic regions of a collection of $V$. dahliae strains, and determined the ability of these strains to infect a collection of plant species that belong to various plant families. We observed that $V$. dahliae strains that are able to infect the same host plant harbour highly divergent LS effector repertoires. Moreover, not even a single LS effector is shared by the strains that are able to infect the same host species. This has led me to suggest that different $V$. dahliae strains infect the same host plant by utilizing different effectors, suggesting redundancy among the activities of effector repertoires between lineages. Furthermore, I assessed the expression of core $V$. dahliae effector genes, and observed considerable differential expression between hosts. I conclude that the variability within LS effector gene repertoires as well as within the expression of core effector genes of $V$. dahliae strains may be instrumental for immunity evasion on particular host plants, allowing the various strains to be competitive in the co-evolution with their hosts.

$V$. dahliae strains are generally characterized by their ability to infect a broad host range, but individual strains display differential capacity to infect particular hosts. I reasoned that this differential host specificity is linked to the presence of host-specific effectors that contribute to disease establishment. In Chapter 4, I conducted comparative genomics of a sunflower pathogenic strain with a collection of non-pathogenic strains. Two effector genes were identified that exclusively occur in the sunflower pathogenic strain and that are highly induced during host colonization. These two identified candidate effector 
genes are identical copies that likely evolved via a segmental duplication. Functional analysis revealed that this duplicated effector gene quantitatively contributes to $V$. dahliae virulence on sunflower, but not on other host plants.

Z. tritici strains exhibit host specificity, as they generally infect either the bread wheat (BW) or durum wheat (DW). In Chapter 5, I aimed to explore genome-wide differences underpinning host specificity in this fungal pathogen. Whole-genome sequencing of a worldwide collection of $136 \mathrm{Z}$. tritici isolates and phenotyping assays on a set of BW and DW cultivars were conducted, revealing extensive genetic diversity amongst $Z$. tritici isolates. Isolates originating from the Middle East, the centre of origin of wheat, showed an increased genetic diversity compared to isolates obtained from other geographical locations. Assessment of genome-wide differences between BW and DW isolates revealed four effector genes carrying non-synonymous single nucleotide polymorphisms in BW isolates. Presumably, extensive genetic diversity among $Z$. tritici isolates is indicative of its evolutionary potential to infect and overcome resistance in wheat plants, which has implications for the control of this fungal wheat disease.

In Chapter 6, I conducted comparative population genomics combined with genetic mapping in a collection of virulent and avirulent $Z$. tritici isolates on the bread wheat cultivar Shafir that carries the resistance gene Stb6. A polymorphic genomic region that determines avirulence was identified, leading to the identification of the avirulence effector gene AvrStb6. Recognition of this effector by Stb6 triggers host resistance. The paradigm states that deployment of Stb6 leads to removal of this avirulence effector gene from pathogen populations. However, when we crossed Z. tritici on wheat, we found that sex occurs even with an avirulent parent, thus leading to the maintenance of AvrStb6 in subsequent populations. This observation suggests that the sexual behaviour of $Z$. tritici has major implications for disease control.

Chapter 7 is the general discussion of this PhD thesis and describes some of the approaches to identify effector genes, how pathogens adapt their effector gene repertoires to their host plants, and how the knowledge on pathogen effectors can be used establish durable and broad-spectrum resistance. 


\section{ACKNOWLEDGEMENTS}

These are the last words in my PhD thesis, which I hope you enjoyed reading. To start, I would like to thank Allah for helping me to successfully finish this thesis. Without him I am nothing and hopefully he will accept this thesis research in the balance of my good deeds.

Surely, I have not established this thesis on my own, but rather with the support of many people including my supervisors, family, colleagues, and friends. To this end, I would like to thank my promotor Bart for his supervision. Without you, Bart, surely I couldn't have done it. I still remember when you guided me to start my PhD with my daily supervisor and co-promotor, Michael, whom I also appreciate very much for his daily support. Michael took me by the hand, and bit by bit he taught me how to carry out my research independently. Thank you, Michael, for that. Also, I would like to thank my other co-promotor Gert. I had very nice conversations with you, Gert, on science, religion, and culture. Definitely, I will miss these fruitful conversations.

I would like to thank my colleagues in the Laboratory of Phytopathology who helped me directly or indirectly to make my thesis into a success. Particularly, I would like to thank my colleagues in the bioinformatics office including Jasper, Sander, Claudio, and Xiaoqian for the nice and fruitful scientific discussions. Surely, in that 'hidden' and 'independent' office we managed to generate a very friendly, cosy, and nice work place and I miss working with all of you. This office also used to house Luigi for a couple of years, and of course I would like to thank him for everything. Additionally, I would like to thank Jinling for providing the collaboration on Chapters 3 and 4 . I would also like to thank Guillaume, Anne, and Marc-Henri for the lovely discussions that we had over Skype on Chapter 5. I would like to thank Paul for his help on Chapter 5. I also would like to thank Amir and Lamia for providing me the data that I needed for the bioinformatics analysis conducted in Chapter 6 and I would like to thank Els for teaching me how to do the phenotyping on wheat leaves. I would like to thank Harold, Jan, Francine, Klaas, and Matthieu for their input in the project during the Friday seminars. Lastly, thank you Ali for your administrative help.

I miss my friends in Wageningen a lot. You, my friends, were always there whenever I needed you. Thank you so much Ali, Asmaa, Mohab, Ahmed, Muniroh, Daniel, Shahid, Fajar, Confidence, Kostas, Miguel, Anna, Elizabeth, Kiflom, Aisha, and Eyup. Without you, it would have not been as much fun to be in Wageningen.

To my lovely parents, may Allah reward you with goodness (jazakom Allah khairan). I could not have done this without your support. Every step that I make towards my success in this life definitely goes back to you. You raised me very well, you always cared about me, and you did everything to make me a successful person. I will always be very grateful to you. 



\section{ABOUT THE AUTHOR}

Hesham A.Y. Gibriel was born in Cairo, Egypt on the $11^{\text {th }}$ of August 1988. After completing his Bachelor studies (2005-2009) with a specialization in Plant Biotechnology at the Faculty of Agriculture, Cairo University, Egypt, he worked as a product manager at Alliance-Global Egypt. Subsequently, Hesham moved to Italy in 2011 to do an MSc in Plant Pathology with the specialization in Plant Virology. After obtaining his MSc degree in 2014, he started his PhD research in the Laboratory of Phytopathology of Wageningen University in the Netherlands under the supervision of Prof. Dr. Bart Thomma. He performed comparative genomics to study host adaptation of the filamentous ascomycete fungi Zymoseptoria tritici and Verticillium dahliae, which resulted this PhD thesis. Since 2018 Hesham is working as a researcher at University College Dublin, performing comparative genomics on $Z$. tritici. 



\section{LIST OF PUBLICATIONS}

Gibriel, H. A. Y., Thomma, B. P. H. J., and Seidl, M. F. 2016. The age of effectors: genomebased discovery and applications. Phytopathology 106:1206-1212.

Kema, G. H. J.\#, Mirzadi Gohari, A. \#, Aouini, L.\#, Gibriel, H. A. Y.\#, Ware, S. B., van den Bosch, F., Manning-Smith, R., Alonso-Chavez, V., Helps, J., Ben M'Barek, S., Mehrabi, R., Diaz-Trujillo, C., Zamani, E., Schouten, H. J., van der Lee, T. A. J., Waalwijk, C., de Waard, M. A., De Wit, P. J. G. M., Verstappen, E. C. P., Thomma, B. P. H. J., Meijer, H. J. G., and Seidl, M. F. 2018. Stress and sexual reproduction affect the dynamics of the wheat pathogen effector AvrStb6 and strobilurin resistance. Nature Genetics 23:678. 

Education Statement of the Graduate School

Experimental Plant Sciences

Issued to: Hesham Gibriel

Date:

20 February 2019

Group: Laboratory of Phytopathology

University:

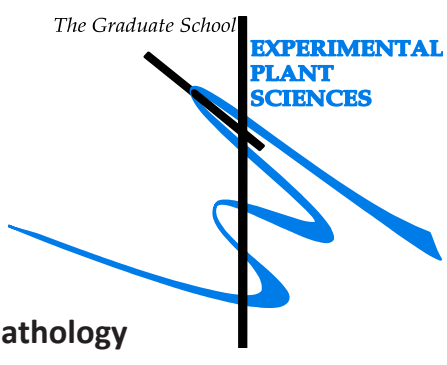

Wageningen University \& Research

\begin{tabular}{|c|c|c|}
\hline \multicolumn{2}{|c|}{ 1) Start-Up Phase } & date \\
\hline \multirow[t]{2}{*}{$\checkmark$} & First presentation of your project & \\
\hline & $\begin{array}{l}\text { Comparative population genomics combined with genetic } \\
\text { mapping promotes effector discovery in the fungal wheat } \\
\text { pathogen Zymoseptoria tritici }\end{array}$ & 27-Nov-2015 \\
\hline \multirow[t]{2}{*}{$\checkmark$} & Writing or rewriting a project proposal & \\
\hline & Comparative pathogenomics of ascomycete fungi & Jul-2015 \\
\hline \multirow[t]{2}{*}{$\checkmark$} & Writing a review or book chapter & \\
\hline & $\begin{array}{l}\text { The age of effectors: genome-based identification and } \\
\text { future applications. Phytopathology 106:1206-1212. DOI: } \\
\text { 10.1094/PHYTO-02-16-0110-FI }\end{array}$ & Mar-2016 \\
\hline \multirow[t]{3}{*}{ 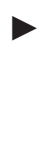 } & MSc courses & \\
\hline & PHP-30306 Plant-Microbe Interactions & May-June-2015 \\
\hline & PHP-30806 Molecular Aspects of Bio-interactions & Jan-Feb-2016 \\
\hline
\end{tabular}

Subtotal Start-Up Phase

15.0 credits*

2) Scientific Exposure
EPS PhD student days
EPS PhD student days 'Get2Gether'
EPS PhD student days 'Get2Gether'
EPS Theme Symposia
EPS Theme 2 Symposium 'Interactions between Plants and
Biotic Agents' + WCS Day
EPS Theme 2: Interactions between Plants and Biotic
Agents
EPS Theme 2: Interactions between Plants and Biotic
Agents
EPS Theme 4: Genome Biology + 'WURomics: Technology-
Driven Innovation for Plant Breeding'

$\underline{\text { date }}$

29-30-Jan-2015

28-29-Jan-2016

20-Feb-2015

22-Jan-2016

23-Jan-2017

15-16-Dec-2016 Driven Innovation for Plant Breeding' 
National meetings (e.g. Lunteren days) and other National Platforms

Annual meeting 'Experimental Plant Sciences', Lunteren

13-14-Apr-2015

BioSB meeting, Lunteren

4-5-Apr-2017

\section{Seminars (series), workshops and symposia}

DTL focus meeting: New sequencing technology:

Nanopore and PacBio

Seminar: Dr. Theo van der Lee, Comparative genomics

of plant pathogens: cryptic sex and the escape of some chromosomes to a genome defence system

Applications and Challenges of Oxford Nanopore Sequencing in the Life Science Industry

BiosB seminar: Bioinformatics, genomics and

metagenomics applied to farm animal health and food security by Mick Watson

B-wise seminar: Marco Bink, Reconstruction of genome ancestry blocks in complex plant populations

B-Wise seminar: Sven Warris, Electronic annotation:

where we are, where we want to be, and semantics to get there

B-wise seminar: Sander Rodenburg, Reconstruction of a metabolic network for the plant pathogen Phytophthora infestans

Seminar: Dr. Sophie Nadot, Perianth evolution in

Ranunculaceae: are petals ancestral in the family?

Seminar: Dr. Martin Cann, The immune receptor Rx1

08-Mar-2016

15-Dec-2016

14-Apr-2016

20-Apr-2016

02-Feb-2016

05-Apr-2016

06-Dec-2016

20-May-2016

remodels chromatin and chromatin interactors in

immunity

Seminar: Frantisek Marec, From sex chromosomes to sex determination in Lepidoptera

Rewriting our genes meeting

11-Jul-2017

\section{Seminar plus}

International symposia and congresses

Zymoseptoria community meeting, Kiel, Germany

05-06-Sep-2017

COST sustain, Banylus sur Mer, France

17-19-Feb-2016

Presentations

Cost sustain (talk)

17-Feb-2016

EPS theme4 (talk)

16-Dec-2016

BioSB (poster)

04-Apr-17

B-Wise seminar (talk)

04-Nov-17 


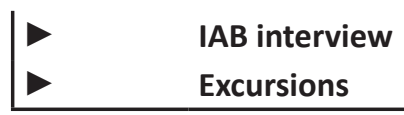

Subtotal Scientific Exposure 10.9 credits*

\begin{tabular}{|lc|}
\hline 3) In-Depth Studies & date \\
& EPS courses or other PhD courses \\
& Postgraduate course: Genome assembly \\
Next Generation Sequencing data analysis & 28-29-Apr-2015 \\
Journal club & $29-31-A u g-2016$ \\
Phytopathology literature discussion group & $2014-2018$ \\
\hline$\quad$ Individual research training & \\
\hline
\end{tabular}

Subtotal In-Depth Studies 4.5 credits*

\begin{tabular}{|lc|}
\hline 4) Personal Development & date \\
Skill training courses & \\
Scientific writing & Feb-Apr-2015 \\
PhD competence assessment & $17-19-$ Feb-2015 \\
EPS Introductory Course & $22-$ Sep-2015 \\
Career Orientation & $4-25-$ Oct-2016 \\
Organisation of PhD student days, course or conference & \\
Membership of Board, Committee or PhD council of EPS & \\
\hline
\end{tabular}

Subtotal Personal Development 3.8 credits*

TOTAL NUMBER OF CREDIT POINTS*

34.2

Herewith the Graduate School declares that the PhD candidate has complied with the educational requirements set by the Educational Committee of EPS, which comprises a minimum total of 30 ECTS credits.

* A credit represents a normative study load of 28 hours of study. 
This work was carried out in the Laboratory of Phytopathology, Wageningen University, The Netherlands.

Financial support from Wageningen University for printing this thesis is greatly appreciated.

Cover \& layout design: Loes Kema | gvo.nl

Printed by: GVO drukkers \& vormgever, Ede (NL) | gvo.nl 
